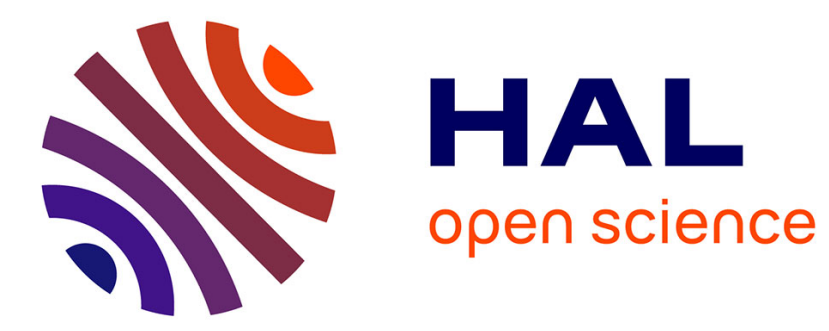

\title{
All-optical synchronization for quantum networking
}

Lufti Arif Bin Ngah

\section{To cite this version:}

Lufti Arif Bin Ngah. All-optical synchronization for quantum networking. Other [cond-mat.other]. Université Nice Sophia Antipolis, 2015. English. NNT : 2015NICE4141 . tel-01292197

\section{HAL Id: tel-01292197 \\ https://theses.hal.science/tel-01292197}

Submitted on 22 Mar 2016

HAL is a multi-disciplinary open access archive for the deposit and dissemination of scientific research documents, whether they are published or not. The documents may come from teaching and research institutions in France or abroad, or from public or private research centers.
L'archive ouverte pluridisciplinaire HAL, est destinée au dépôt et à la diffusion de documents scientifiques de niveau recherche, publiés ou non, émanant des établissements d'enseignement et de recherche français ou étrangers, des laboratoires publics ou privés. 


\section{UNIVERSITÉ NICE SOPHIA ANTIPOLIS - UFR Sciences \\ ÉCOLE DOCTORALE EN SCIENCES FONDAMENTALES ET APPLIQUÉES}

\section{Ph.D Dissertation}

to obtain the title of

\section{Docteur en Sciences}

of Université Nice Sophia Antipolis (UNS), France.

Specialization : PHYSICS

Presented by

\section{Lutfi Arif BIN NGAH}

\section{All-optical synchronization for quantum networking.}

Synchronisation toute optique d'un réseau de communication quantique.

Defended on December 11, 2015

Jury :

$\begin{array}{llll}\text { Reviewers: } & \text { Sara DUCCI } & \text { Professor } & \text { Université Paris Diderot - Paris } 7 \\ & \text { Rosa TUAlle-Brouri } & \text { Professor } & \text { Inst. Optique Graduate School, Palaiseau } \\ \text { Examinator: } & \text { John G. RARITY } & \text { Professor } & \text { University of Bristol (UK) } \\ \text { Advisors: } & \text { Sébastien TANZILli } & \text { DR CNRS } & \text { Université Nice Sophia Antipolis } \\ & \text { Virginia D'AURIA } & \text { MCF } & \text { Université Nice Sophia Antipolis }\end{array}$





\title{
All-optical synchronization for quantum networking.
}

\begin{abstract}
This manuscript reports the development of fundamental resources for long distance quantum communication based on fibre telecom technology and non-linear optical waveguides. After a general introduction on quantum communication, the thesis is structured along three parts.

The first part illustrates the development of two photonic polarization entanglement sources suitable for quantum networking. Both sources allow the generation of paired photons in the telecom C-band of wavelengths via spontaneous parametric down conversion (SPDC) in periodically poled lithium niobate waveguides (PPLN/W). The first source relies on type-II phase matching. In this configuration, two important elements, namely fiberbased walk-off compensation, and deterministic separation of the paired photons using lowloss, dense wavelength division multiplexing (DWDM) filters, are detailed. On the other hand, the second source relies on type-0 SPDC process. Thanks to advanced filtering and interferometric strategies, the produced paires photons can be manipulated at will regarding both their spectral bandwidth and entanglement state, respectively. The source is therefore adapted to a broad variety of quantum photonics applications.
\end{abstract}

In the second part, two high quality heralded single photon sources (HSPS) are highlighted. The first HSPS relies on on-chip generation and spatial multiplexing of heralded C-band telecom single photons towards achieving higher bit rates. This source relies on the hybridization of two enabling technologies, namely four PPLN/Ws integrated on a single substrate for photon generation by SPDC and laser-written waveguide technologies for routing and multiplexing purposes. Interestingly, such a hybridization has never been demonstrated before. The second HSPS takes advantage of passive temporal multiplexing of a single SPDC process. By taking advantage a $10 \mathrm{GHz}$ repetition rate telecom laser and DWDM filters, combined with an efficient type-0 PPLN/W, an ultra-fast HSPS emitting single photons in the $\mathrm{MHz}$ regime at a telecom wavelength is demonstrated.

Finally, an all-optical approach towards efficient and accurate synchronization of remote entangled photon pair sources within quantum relay architecture over long distances is presented. This particular synchronization technique highlights the use of ultra-fast 
picosecond pulsed telecom fiber laser, operating at $2.5 \mathrm{GHz}$ repetition rate, acting as a master optical clock, enabling to accurately synchronize the emission of photon pairs in the telecom C-band of wavelengths at two remote locations. This innovative approach is applied for synchronizing two remote PLLN/W based sources operated at $2.5 \mathrm{GHz}$, and preliminary results on two-photon interference obtained with single photons coming from each source are shown and discussed.

Keywords: quantum communication, quantum networking, integrated non-linear optics, quantum optics, PPLN waveguides, polarization entanglement, heralded single photon source, quantum interference, quantum relay. 


\section{Synchronisation toute optique d'un réseau de communication quantique.}

Résumé : Ce manuscrit expose le développement de ressources fondamentales pour les communications quantiques à longues distances basées sur les technologies des fibres optiques télécoms et des guides d'onde optiques non linéaires. Après une introduction générale sur les communications quantiques, cette thèse est structurée en trois parties principales.

La première partie illustre le développement de deux sources d'intrication photonique en polarisation adaptées aux réseaux quantiques. Les deux sources permettent la génération de paires de photons dans la bande de longueur d'onde $\mathrm{C}$ des télécoms via la conversion paramétrique spontanée (SPDC) dans des guides d'ondes non linéaires intégrés sur niobate de lithium périodiquement polarisé (PPLN/W). La première source s'appuie sur un accord de phase de type-II. Dans cette configuration, sont détaillés deux éléments importants, à savoir la compensation par une fibre optique de la biréfringence induite dans le cristal non linéaire, et la séparation déterministe des paires de photons par le biais de filtres à démultiplexage en longueur d'onde (DWDM) à faibles pertes. La deuxième source repose sur un accord de phase de type-0. Dans cette configuration, grâce à des solutions de filtrage et d'interférométrie avancées mises en place après le cristal non linéaire, les photons produits peuvent être manipulés à volonté, aussi bien en terme de largeur spectrale que d'état d'intrication. La source est par conséquent adaptée à une grande variété d'applications en photonique quantique.

Dans la seconde partie, sont discutées les réalisations de deux sources de photons uniques annoncés (HSPS) de grande qualité. La première s'appuie sur la génération et le multiplexage spatial sur puce de photons uniques annoncés dans la bande $\mathrm{C}$ des télécoms dans le but d'atteindre des taux de génération accrus. Cette source repose sur l'hybridation de deux technologies clé, à savoir quatre guides d'onde non linéaires intégrés sur une même puce pour la génération des photons et la technologie d'écriture de guides d'onde par laser pour le routage et le multiplexage. Notons que ce type d'hybridation n'avait jamais été démontré auparavant. La seconde HSPS exploite le multiplexage temporel passif d'un processus SPDC unique. En effet, l'utilisation d'un laser télécom cadencé à $10 \mathrm{GHz}$ et de filtres DWDM, combinés à un guide d'onde non linaire de type-0, a permis d'implémenter une source ultra-rapide émettant des photons uniques à des cadences de l'ordre de quelques 
$\mathrm{MHz}$ dans la bande $\mathrm{C}$ des télécoms.

Enfin, nous présentons une approche tou-optique visant la synchronisation efficace et précise de sources distantes de paires de photons intriqués, agencées selon une architecture de type relais quantique distribué. Cette technique de synchronisation innonvante met en avant l'utilisation d'un laser télécom impulsionnel picoseconde cadencé à $2,5 \mathrm{GHz}$ en tant qu'horloge optique de référence. Cette horloge autorise la synchronisation précise de l'émission de paires de photons dans la bande $\mathrm{C}$ des télécoms en deux lieux distants. Cette approche est appliquée à la synchronisation de deux sources basée sur des guides d'onde non linéaires opérant à $2,5 \mathrm{GHz}$. À ce titre, des résultats préliminaires d'interférence à deux photons obtenue à partir de photons uniques en provenance de chacune de ces sources sont montrés et discutés.

Mots clés : communication quantique, réseaux quantiques, optique intégrée, optique quantique, guides PPLN, intrication en polarisation, source de photons uniques annoncés, interférence quantique, relais quantique. 


\section{Acknowledgements}

First and foremost I would like to express my special appreciation and my most heartfelt thanks to my main thesis supervisor, Sébastien Tanzilli for being my mentor and also a very good friend. I wholeheartedly appreciate everything you've done for me, everything. To my thesis co-director, Virginia D'Auria, I will never forget the time we spent working together in the CONNEQT lab. I will always remember your kindness, and our tea time together with Bruno! Olivier Alibart, you taught me a lot of things, in the lab, as well as in Sydney. Laurent Labonté, I really appreciate your kindness and all your helps. Not to forget Dan Ostrowsky for all the hot sauces, and the lunches at your wonderful place. Thanks Dan. To Anders Kastberg who also happens to be a Chelsea fan like me, I appreciate all the discussions and insights from you. To Eric Picholle, I still remember the late evenings we spent in the lab, preparing me for the thesis defense. Séb, Virginia, Olivier, Laurent, Anders and Eric, thank you very much. All of you have been there to support me and words cannot express how grateful I am to you guys.

I would also like to thank the members of my dissertation comittee: Prof. Sara Ducci, Prof. Rosa Tualle-Brouri and Prof. John G. Rarity for their time and insightful comments.

Florian Kaiser, I still remember the day when I first met you in the lab. During my time in LPMC, I learned a lot from you. We also shared a lot of unforgettable moments together, memories that I will always cherish. Florent Doutre, Anthony Martin, Amandine Issautier, Djeylan Aktas, Bruno Fedrici , Panagiotis Vergyris, Tommaso Lunghi, Michele Traetta, Marco Bentivegna, Florent Mazeas, Mathieu Pellegrin, Julian Böehm, Nahed Sihem Bounoua, Chiara Scognamiglio, Martina Iapichino, Jean-Bernard Duchez, Jean-François Lupi, Manuel Vermillac, Alexandre Cohen and others, you guys are the best! Thank you very much for letting my stay in LPMC be an enjoyable moment.

Also, I would like to thank the director of LPMC, Fabrice Mortessagne, the administrative team comprising Nathalie Koulechoff, Christine Chassagneux and Christine BoudardMorteau, programming and IT experts Bernard Gay-Para, Grégory Sauder and Jérôme Mauro, the masters of electronics and mechanics Frédéric Juan, Frédéric Lippi, Christophe Pitiot, Jean-Luc Vanni, and to the lovely chemistry expert Yaroslava Izmaylov. Also, a special thanks to Jean-Pierre Romagnan for all the helps and advises on Helium. 
Last but not least, I thank my wife Raja Fazliza Raja Suleiman and my parents, Ngah Bidin and Che Ku Zawiah Binti Che Ku Ibrahim, for all of the sacrifices that they have made on my behalf. Not to forget my younger brothers and sisters (seven of them), and my housemates in France Dr. Nazrol, Naji, Asyraf (Bo), Hisham, Zul Farid, Izzat, and Isham, who believed in me.

Thank you. 


\section{Contents}

I Fundamental aspects of quantum communication $\quad 9$

1 Optical Communication: from the classical to the quantum world 11

1.1 Classical information and communication . . . . . . . . . . . . 11

1.2 Quantum communication ... . . . . . . . . . . . . . . 19

2 Towards long-distance quantum communication $\quad 47$

2.1 Pushing the "quantum" distance . . . . . . . . . . . . . . . . . . . 47

II Polarization entanglement generation at telecom wavelength $\quad 59$

$\begin{array}{lll}3 & \text { Advances in polarization entangled photon pair generation } & 61\end{array}$

3.1 Advances in polarization entanglement via SPDC . . . . . . . . . . . . 61

3.2 The relevance of polarization entanglement . . . . . . . . . . . 65

3.3 A quick recap on generating photon pairs via SPDC in PPLN/W . . . . . . 67

4 Compact polarization entanglement source based on a type-II PPLN/W 71

4.1 Motivation: quantum key distribution . . . . . . . . . . . 71

4.2 Compact polarization entanglement generator: the experiment . . . . . . . . 72

4.3 Conclusion and perspectives . . . . . . . . . . . . . . . . . 97

5 Versatile polarization entanglement source based on a type-0 PPLN/W 99

5.1 Motivation: a quest for "versatility" . . . . . . . . . . . . . . . . . 100

5.2 Versatile polarization entanglement source: the experiment . . . . . . . . . 100

5.3 Conclusion and perspectives . . . . . . . . . . . . . . . . . . . . . . . 121

III High-rate heralded single photon sources at telecom wavelength 123

6 Prominent strategies for producing single photons $\quad \mathbf{1 2 5}$

6.1 Single photon sources: state-of-the-art . . . . . . . . . . . . . . . . . 125

6.2 Gauging the quality of an HSPS . . . . . . . . . . . . . . . 131

6.3 Battling the multi-photon . . . . . . . . . . . . . . 136

7 Spatially multiplexed single photons through hybridization 139

7.1 Uniting FLDW and PPLN $/ \mathrm{W}$. . . . . . . . . . . . . . . . . . . . . . 139

7.2 Experimental arrangement highlighting hybridization . . . . . . . . . . . . 144

7.3 Experimental results and discussion . . . . . . . . . . . . . . . . 147

7.4 Conclusion and perspectives of the source . . . . . . . . . . . . . . 152 
8 Ultra-fast heralded single photon source based on telecom technologies $\mathbf{1 5 5}$

8.1 Inspirations from the telecom industry . . . . . . . . . . . . . . . 155

8.2 Ultra-fast generation of heralded single photons: the experiment . . . . . . . 157

8.3 Characterizing the quality of single photon sources via HBT . . . . . . . . 166

8.4 Conclusion and perspectives . . . . . . . . . . . . . . . . . . . 170

IV Quantum networking based on entanglement swapping 173

9 All-optical synchronization for quantum networking $\quad \mathbf{1 7 5}$

9.1 Theoretical framework . . . . . . . . . . . . . . . . . . . . . 176

9.2 The quantum relay dilemma . . . . . . . . . . . . . . . . . . . . . . . . . 179

9.3 Our approach: all-optical synchronization . . . . . . . . . . . . . . . . . 181

9.4 Preliminary tests . . . . . . . . . . . . . . . . . . . . . 188

9.5 Towards the first prototype of HOM experiment . . . . . . . . . . . . . . . . 191

$\begin{array}{ll}\text { Overall summary } & 205\end{array}$

A Single photon detectors and coincidence electronics 209

A.1 InGaAs avalanche photo-diodes: working principle . . . . . . . . . . . . . 209

A.2 Superconducting single photon detectors: working principle . . . . . . . . 210

A.3 Figures-of-merit and comparison . . . . . . . . . . . . . . . . . . 212

A.4 Coincidence detection scheme . . . . . . . . . . . . . . . . . . . . . 213

$\begin{array}{ll}\text { Bibliography } & 215\end{array}$ 


\section{General Overview}

A brief overview, from the birth of quantum physics

to the exploitation of entanglement in quantum communication.

Disregarded by some, loved by some, and admitted by many. This statement perfectly describes quantum physics, from the time of its infancy up to nowadays. This is not surprising considering all the weird phenomena that it predicts, notably coherent superposition and entanglement, which have no common-sense, i.e. classical explanation. Despite the doubt from a number of world-renowned physicists, including Albert Einstein, the quantum theory backers, led by Niels Bohr and Werner Heisenberg (among others), had somehow managed to refute every single criticism expressed against quantum physics. Progressive debates between Einstein and Bohr since the early 1920s was somehow stopped in 1935 when Einstein and his co-workers proposed an important challenge to the quantum community through one of the most influential paper of the $20^{\text {th }}$ century entitled "Can quantum-mechanical description of physical reality be considered complete?" [Einstein et al. 1935]. Ironically, beyond schrödinger's insight on entanglement, it was no other than Einstein, Podolsky and Rosen (EPR) who first went deep inside the question of entanglement. Among others, EPR trio introduced the concept of local hidden variables in order to have possible explanation for the strong correlations that could be revealed using entangled particles. Being caught off-guard with this new implication of quantum theory, it took months for Bohr to come forward with his arguments refuting the idea of EPR. Since this challenge (known today as the EPR paradox), Einstein was no longer active in defending his views. Nonetheless, during his lifetime, all the debates were purely philosophical. Only in 1964, John S. Bell took Einstein's arguments very seriously and proposed a way to verify the existence, or not, of these so-called local hidden variables [Bell 1964]. This was the kick-off point where physicists became more motivated than ever to test for themselves, the new found inequality. Five years later, John Clauser, Michael Horne, Abner Shimony, and Richard Holt proposed a way to obtain entangled photon pairs, emitted in an atomic cascade of calcium. They also outlined the experimental procedure for the violation of the Bell's inequality with the generated 
entangled photons [Clauser et al. 1969]. Clauser himself, together with Stuart Freedman, demonstrated, for the very first time, such a violation [Freedman \& Clauser 1972]. Just a few years later, the most convincing violations of Bell's inequality were demonstrated in Orsay between 1981 and 1982 by Alain Aspect, Philippe Grangier, Jean Dalibard and Gérard Roger [Aspect et al. 1981, Aspect et al. 1982a, Aspect et al. 1982b]. However, the quest has not stopped there since people started questioning the possible loopholes of Bell's inequality tests. As an answer to non-believers, very recently, the Delft experiment has succeeded in violating the Bell's inequality in a loophole-free environment [Hensen et al. 2015].

A year after the historic Orsay experiment, quantum physics finally met information science. A seminal paper written in 1984 by Charles Bennett and Gilles Brassard, entitled "Quantum cryptography: public key distribution and coin tossing" had give birth to BB84, standing as the very first quantum key distribution protocol (QKD) [Bennett \& Brassard 1984]. Without doubt, this marked the beginning of the quantum communication era [Gisin et al. 2002]. In particular, quantum communication deals with transferring qubits to a remote locations, with the principal objective of implementing QKD protocols over long distances. All over these years, different types of QKD protocols have been proposed, from a simple one way protocol exploiting single photons [Bennett \& Brassard 1984, Scarani et al. 2004a, Gisin et al. 2004, Lo et al. 2005, Stucki et al. 2009, Scarani et al. 2009] to the use of entanglement as enabling resource [Ekert 1991, Gisin et al. 2002, Scarani et al. 2004b]. With QKD, truly random secret keys can be established between two users at remote locations within the possibility of detecting the presence of an eavesdropper. Besides QKD, single photons are heavily exploited, namely in linear optical quantum computing [Knill et al. 2001, Tillmann et al. 2013, Broome et al. 2013] and in quantum metrology [Polyakov \& Migdall 2009, Avella et al. 2011]. We have seen how researchers put some tremendous efforts toward producing high quality single photons. Along the line, single quantum emitters have been employed, so as to emit only one photon at a given time window. Theses type of emitters include quantum dots [Santori et al. 2001, Press et al. 2007] and nitrogen-vacancy (NV) center in diamonds [Tamarat et al. 2006, Mizuochi et al. 2012]. We also have seen single photons being produced, notably at telecom wavelength, by heralded single photon sources (HSPS) based on spontaneous parametric down conversion process in non-linear crystals [Fasel et al. 2004, Alibart et al. 2005, Pomarico et al. 2012,Krapick et al. 2013,Ngah 
et al. 2015]. Entanglement, on the other hand, continues to be one of the major enabling resource for various quantum applications, including also fundamental tests in quantum optics [Kaiser et al. 2012a, Peruzzo et al. 2012]. In the context of long distance quantum communication, entanglement is mandatory in enabling entanglement swapping in quantum relay configuration [Collins et al. 2005,Zukowski et al. 1993, Gisin et al. 2002, Jennewein et al. 2002, Aboussouan et al. 2010]. Moreover, the recently introduced DLCZ protocol [Duan et al. 2001] has successfully called for the development of high quality, narrow band, polarization entangled photon pair sources [Kuklewicz et al. 2006, Piro et al. 2009, Kaiser et al. 2013].

We have seen how vibrant is the field of quantum communication. Quantum communication has somehow attracted a lot of attention in just 30 years. Since its earlier days, much effort has been devoted to various aspects of this field. Already, we have seen some commercial quantum communication devices, namely integrated QKD solutions and quantum random number generators by idQuantique have been used in real world applications. This manuscript particularly focuses on the domain of quantum communication. Throughout it, we propose several, fully guided-wave, quantum experiments by exploiting photon pairs emitted in the telecom C-band of wavelengths, generated using periodically poled lithium niobate waveguides and widely manipulated using standard fiber optical components.

Content of this manuscript, a brief outline of the following chapters.

Part I Fundamental aspects of quantum communication

Chapter 1: Optical Communication: from the classical to the quantum world.

The first chapter of this thesis gives a brief discussion focused on the basics of classical and quantum information sciences. Starting with the classical world, the notion of "bit" which represents the most fundamental unit of classical information is recalled. Furthermore, security issues concerning classical communication are also mentioned. Then, the discussion shifts toward the field of quantum communication where the quantum bit counterpart, the so-called qubit, is described. The most intriguing quantum phenomenon of 
entanglement is also subsequently entailed. In the context of quantum communication, the advantages offered by quantum physics are summed up with the introduction of quantum key distribution (QKD) protocols.

Chapter 2: Towards long-distance quantum communication.

In this chapter, we first outline several factors that limit the distance of which a secret key can be established by QKD. We then highlights the concept of quantum relay such that the distance of a quantum channel can be further increased using entanglement as enabling resource. Furthermore, we compare different forms of quantum communication by calculating their expected secret key rate as a function of the distance separating two communicating parties, say Alice and Bob. Last but not least, enabling quantum protocols required for quantum relay configuration, namely quantum teleportation and entanglement swapping, are also discussed.

Part II Polarization entanglement generation at telecom wavelength

Chapter 3: Advances in polarization entangled photon pair generation.

This chapter is mainly focused on the prior art of photonic polarization entanglement sources. The quest of polarization entangled photons from the early days of atomic-cascade decays until the era of integrated photonics is described. Along the line, this chapter also cites various efforts that have been carried out in photonic polarization entanglement engineering, notably from different type of non-linear interaction of spontaneous parametric down conversion (SPDC) in non-linear crystals. In parallel, a quick description on the generation of photon pairs via SPDC in periodically poled lithium niobate waveguides $(\mathrm{PPLN} / \mathrm{W})$ is presented.

Chapter 4: Compact polarization entanglement source based on a type-II PPLN/W.

The development of photonic polarization entanglement sources for future quantum networking solutions stands as the main aim of this chapter. We basically highlights a compact and practical approach for generating high-quality polarization entanglement in a fully guided-wave fashion. Such a source benefits from photon pair generation in the 
telecom C-band of wavelengths via SPDC in type-II PPLN/W. Within this framework, two important elements, namely fiber-based walk-off compensation, and deterministic separation of the paired photons in two adjacent telecommunication channels using low-loss, dense wavelength division multiplexing (DWDM) filters, are detailed. Eventually, excellent entanglement quality is observed through Bell-CHSH inequality test [Kaiser et al. 2012b].

Chapter 5: Versatile polarization entanglement source based on a type-0 PPLN/W.

With this chapter, another high-quality, telecom-wavelength, polarization entangled photon pair source is considered and demonstrated. Considered versatile, the photons produced by this particular source can be manipulated, in term of both their spectral bandwidth, and entanglement state, to be adapted to broad variety of quantum applications, namely QKD and atomic ensemble-based quantum memory devices. On the contrary to the development outlined in Chapter 2, we rely in this chapter on the use of type-0 SPDC in PPLN/W toward generating photon pairs. This chapter also outlines the engineering of photonic entanglement from a co-polarized photon pair using an advanced interferometric solution. As a proof for high-quality entanglement, the outcomes from Bell-CHSH inequality tests are also presented [Kaiser et al. 2013].

Part III High-rate heralded single photon sources at telecom wavelength

Chapter 6: Prominent strategies for producing single photons.

This chapter highlights various strategies toward producing pure single photon states, notably the use of single quantum emitters such as quantum dots and nitrogen-vacancy center in diamond, and in particular the heralding technique benefiting from two simultaneously generated photons. This chapter focuses on the latter, i.e. the so-called heralded single photon sources (HSPS). Then the general method of Hanburry Brown and Twiss enabling to infer the singleness of the produced single photons, via the second-order correlation function of light $g^{(2)}(0)$, as well as the notion of coincidence-to-accidental ratio $(C A R)$, are outlined [Hanbury Brown \& Twiss 1956]. We note that both $g^{(2)}(0)$ and $C A R$ represent the figures-of-merit, gauging the quality of single photon sources. To finish, this chapter proposes some possible ways of improving the performances of such sources. 
Chapter 7: Spatially multiplexed single photons through hybridization.

This chapter stresses not only the generation of C-band telecom single photons via spatial multiplexing of several PPLN/Ws, but also on the hybridization aspect combining two different prominent technologies in the field of integrated optics permitting the development of such sources. By taking advantage of both PPLN/W and femtosecond laser direct-write (FLDW) integrated photonic chips, a compact, spatially multiplexed HSPS is demonstrated. Interestingly, such hybridization has never been demonstrated before. Besides the type-0 PPLN/W as the workhorse for generating photon pairs, two integrated FLDW chips are also discussed in this chapter. Moreover, this chapter also outlines the use of fast optical switching strategy in order to correctly route the correct heralded photons. The performance of the source is gauged upon $C A R$ measurements and the corresponding results are discussed [Meany et al. 2014].

Chapter 8: Ultra-fast heralded single photon source based on telecom technologies.

The quest for high quality HSPS is continued in this chapter. Based on a different approach compared to that of Chapter 6, we highlight passive temporal multiplexing of a single SPDC process. By taking advantage a $10 \mathrm{GHz}$ repetion rate telecom laser and DWDM filters, combined with an ultra-efficient type-0 photon pair generator based on a PPLN/W, an ultra-fast HSPS emitting single photons in the $\mathrm{MHz}$ regime at a telecom wavelength is demonstrated. Moreover, the single photon character of the source is inferred by measuring the second-order autocorrelation function $g^{(2)}(0)$ [Ngah et al. 2015].

Part IV Quantum networking based on entanglement swapping

Chapter 9: All-optical synchronization for quantum networking.

This final chapter represents the last major experimental work of this thesis. The aim here is to demonstrate an all-optical approach towards efficient and accurate synchronization of remote entangled photon pair sources within quantum relay architecture over long distances. This chapter highlights the use of ultra-fast picosecond pulsed telecom laser, operating at $2.5 \mathrm{GHz}$ repetition rate, acting as a master optical clock, enabling to accurately 
synchronize the emission of photon pairs in the telecom C-band of wavelengths at two remote locations. Within this chapter, a HOM interference experiment is demonstrated so as to validate our proposed synchronization scheme. 

Part I

Fundamental aspects of quantum communication 



\section{Optical Communication: from the} classical to the quantum world

\section{Contents}

1.1 Classical information and communication . . . . . . . . . 11

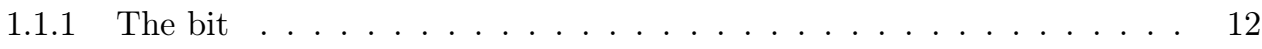

1.1.2 Optical communication . . . . . . . . . . . . . 12

1.1.3 Classical approaches for secure communication, a very brief description 15

1.2 Quantum communication . . . . . . . . . . . . . . 19

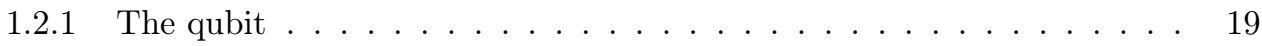

1.2 .2 Cloning a qubit . . . . . . . . . . . . . . . . . . . 22

1.2.3 Entanglement in bipartite quantum systems . . . . . . . . . . . . 25

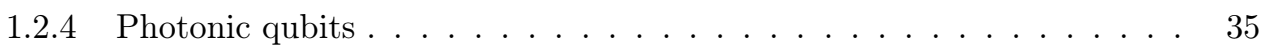

1.2.5 Secret key establishment, "quantumly" . . . . . . . . . . . . . . 40

\subsection{Classical information and communication}

In our "modern" society, the way we live is in constant evolution, such that it not only influences the way we communicate, but also the very way we see the information. Through massive technological advances, we have seen revolutionary methods in communicating and processing information. From radio broadcast to the use of fiber optical networks, from fluctuating analog signals to the digitization, from bulk to pocket-size devices, from oldschool magnetic tapes to high capacity solid-state storage devices, we are indeed living the marvel of information age. 


\subsubsection{The bit}

Older communication systems used to rely on analog signals. These analog signals are transferred from a sender to a receiver through a medium that is known to be the channel of communication. Depending on the carrier, the channel can be anything ranging from atmospheric elements such as air and water, to wire-like structures of coaxial cable and optical fibers. In reality, transmission of information within the communication channel is unpredictable. Due to channel impurities, the signals can be heavily distorted resulting in false interpretation of information by the receiver. To battle signal distortions in such hostile environments, the digitization was introduced. Digitization consists in introducing a binary representation of analog signals. A unit of digital information can only take a single binary value, either 0 or 1 , and this is what is famously known as bit (binary digit). For instance, bits of information can be encoded with the reference to low (0) and high (1) voltage level in telephony electrical circuitry. In optical communication, this is equivalent to the luminosity level in a light pulse (see Fig.1.1 (a)). Information transferred or processed in this way is normally immune to the channel impurities.

\subsubsection{Optical communication}

Since the advent of the single mode optical fibers, related standard telecom components, and conventional computers, the way we communicate and process information have completely revolutionized our society. Today, high-speed Internet comes in (almost) every home, and almost everyone has a personal computer. Communication and data processing fluxes are considerable, as are data storage capacities. To reach this level of maturity, major technological advances have emerged in many areas related directly or indirectly to Physics, from both its fundamental and applied aspects.

On one hand, through the use of semiconductor devices, lasers have been miniaturized (see Fig.1.1 (b)) and microprocessors have seen their speed constantly augmented. In order to synchronize information transfer, these lasers, operating initially in the in continuouswave regime, are coupled to intensity modulators made of integrated optics (see Fig.1.1 (c)). On the other hand, optical fiber technology (see Fig.1.1 (d)) allowed establishing real data distribution channels due to low propagation loss experienced by telecom wavelengths. 

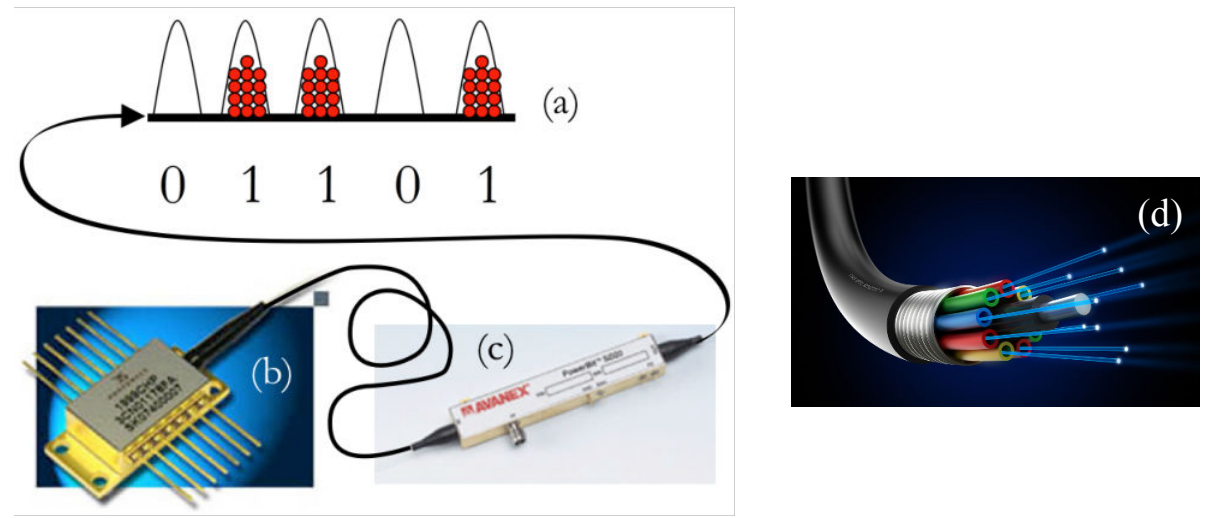

(f)
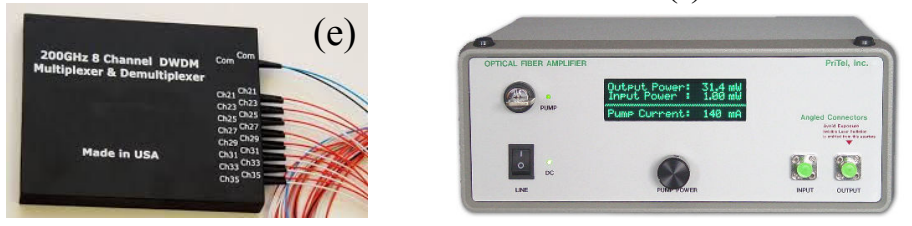

(g)

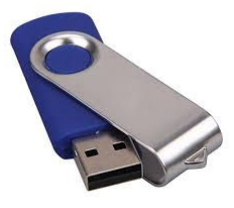

Figure 1.1: Conventional devices used in standard optical communication. (a): Representation of bits in the form of luminosity level contained in optical pulses. (b): An example of widely used, semiconductor diode laser, emitting light in telecom range of wavelength. (c): High-speed optical modulator used for information encoding. (d): Conventional lowloss telecom optical fibers. (e): High-performance and low-loss dense wavelength division multiplexing (DWDM) device. (f): Erbium doped fiber amplifier (EDFA). (g): Pocket-size USB storage device. 
Today these channels are standardized (ITU standard for International Telecommunication Union) and allow, in the telecom $\mathrm{C}$ band (1530-1565 nm), actual wavelength multiplexing and demultiplexing of the conveyed data. This is referred to as dense wavelength division multiplexing (DWDM), see Fig.1.1 (e), and there are up to 70 available channels, spanning each $125 \mathrm{GHz}$ ( $\leftrightarrow 1 \mathrm{~nm}$ ). By combining the capabilities of DWDMs, the possibility to compensate the effects of chromatic dispersion in the fibers, and the advent of optical pulse fiber amplifiers (called erbium doped fiber amplifiers (EDFA), see Fig.1.1 (f)), information data fluxes are nowadays considerable. Commonly achieved rates in standard optical communication links scale up to $40 \mathrm{Gbits} / \mathrm{s}$, and current prototypes already reach the Tbits/s! As such, Fig.1.2 shows the operating principle of an optical DWDM broadband link. Finally, it is possible to store all this data in ever-augmented capacity systems, as is the case of solid-state storage devices and USB sticks (see Fig.1.1 (g)).

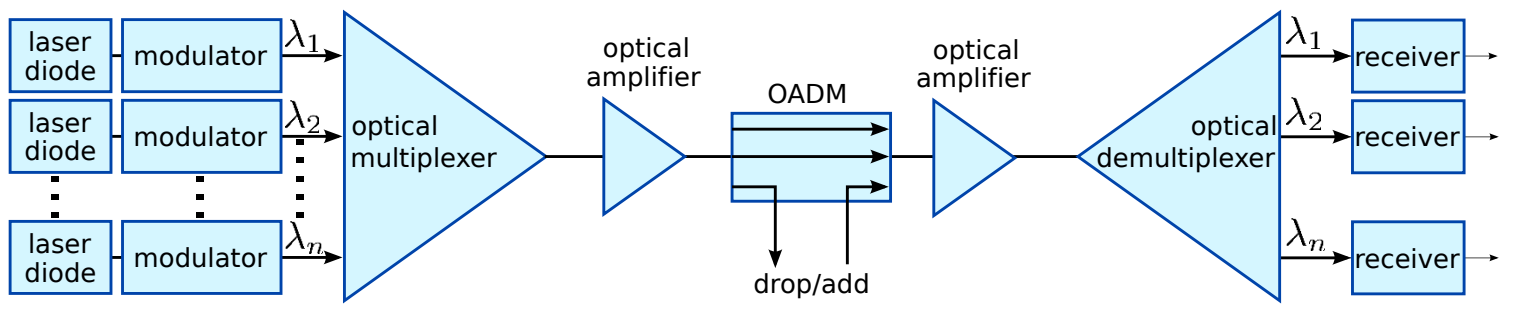

Figure 1.2: Schematic showing the principle of broadband DWDM communication link. A series of high-performance telecom lasers emit each continuous light in their respective wavelengths $\lambda_{1} \cdots \lambda_{n}$. The intensities of these lasers are then modulated via high-speed modulators. Lights propagate in several different wavelengths are eventually multiplexed by a DWDM (see Fig.1.1 (d)), and they are recombined in a single mode optical fiber. About every $500 \mathrm{~km}$, an optical amplifier is positioned in the communication channel so as to compensate for unwanted losses and chromatic dispersion due to fiber-based distribution. Interestingly, an optical add/drop module (OADM) can be used to extract or subtract, any carrier within the multiplexed channel. At the end of the line, a second DWDM is used to demultiplex the incoming signals and proper redistribution of the carrier to final destination is carried out.

If there is a take-home message to be remembered, out of the above discussion, we can for sure say that everything is possible with today's optical communication, and processing of classical information. As a matter of fact, we know how to generate information using semiconductor laser coupled to integrated intensity modulators, distribute it over virtually unlimited distances via optical amplifiers and pulse amplifiers, store it without loss, with an efficiency of $100 \%$ and over quasi-infinite time, handle and treat it at will. In particular, 
Fig.1.3 provides a map (probably non exhaustive) of all optical undersea cables linking the five continents on Earth.

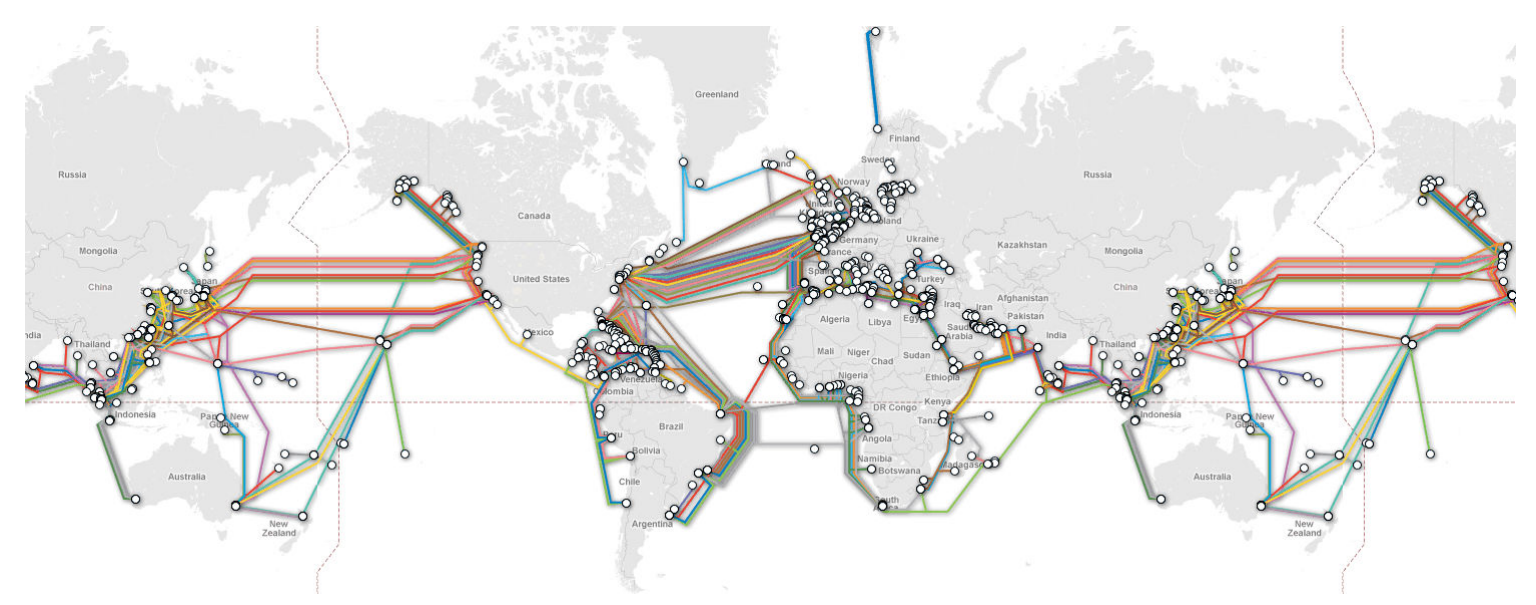

Figure 1.3: Fast digital connectivity across five continents via undersea fiber optical networks.

We will see all along this manuscript that quantum communication is still far away for the above mentioned classical network performances. However, the field is advancing rapidly. The problem we are facing lies in the very way quantum information is coded, namely that it has to be manipulated coherently in order to protect its quantum essence. This is why stringent technical problems have to be addressed for future developments of long-distance and high-speed quantum communication networks. However, quantum information is a very broad research area, spanning photonics, atomic physics, superconductors, crystals, etc., and its future probably lies in the marriage of the most advanced technologies out of both fundamental and applied physics research laboratories.

\subsubsection{Classical approaches for secure communication, a very brief de- scription}

Today, digitization is everywhere. For instance, an instant messaging or skype session between family members in Kuala Lumpur and Nice is done in a fully digital fashion. The same applies for uploading a document to a cloud server based in San Francisco. So, the only question left open is whether we are capable to ensure the security of our digital information at hand.

Among other things, history tells us so much about previous civilizations. As we are 
now today, they were also concerned with the necessity of securing the information and their ingenious methods dealing with such issue have laid the foundation of the art of cryptography. In its simplest form, cryptography consists of writing messages, in such a way that only a few can understand the underlying hidden information. In the ancient times, the Egyptians already used hieroglyphics as early as 1900 B.C. [Singh 2000]. Once considered to be bullet-proof, the mystery of ancient hieroglyphics was finally understood with the discovery of Rosetta Stone in 1799. In ancient Greece, the "scytale cipher" or "transposition cipher" was used in Sparta around the $5^{\text {th }}$ century B.C. It involves the use of a leather band spool written with alphabets and a cylinder having flat rectangles. For the scytale cipher, the hidden message is revealed when the leather band is put in place, over the cylinder. This is what we identify as the "algorithm". Meanwhile, the diameter of the cylinder and the size or period of the flat rectangles represent the "cipher key" that needs to be secretly shared between two communicating parties. Later, in the $1^{\text {st }}$ century B.C., a sort of "substitution cipher" was frequently used by Julius Caesar where the nominal alphabet is replaced by an alphabet located further down the sequence (algorithm). Therefore, the shifting size (cipher key) needs to be agreed between the sender and the receiver beforehand. The improvement of such substitution cipher would be the use of random shifting and this is what has been achieved in the middle ages with the introduction of the cipher of Mary Queen of Scots and Vigenère ciphers. However, such substitution ciphers can be easily cracked with the frequency analysis of letters redundancy. This method of analysis was pioneered in the $9^{\text {th }}$ century by a renowned Arab polymath, Al-Kindi from Iraq. Due to his contributions, he is highly regarded as the father of cryptanalysis.

The use of algorithm and cipher key still remain as the foundation of modern cryptography. Compared to the ancient times, the applied algorithm becomes more and more sophisticated. For instance, complex electro-mechanical rotor couplings are used as the algorithm in the Enigma machine during the World War II. Today, the use of machines in cryptography has been replaced by computers as we are benefiting from integrated logic circuits, e.g. microprocessors, for fast digital information processing. Advantageously, any imaginable superior algorithms can be constructed from just mere lines of programming codes. Furthermore, the problem of distributing keys which represents the biggest concern since ancient times has been circumvented by the introduction of public-key cryptosystem. 


\section{Public key encryption: the RSA protocol}

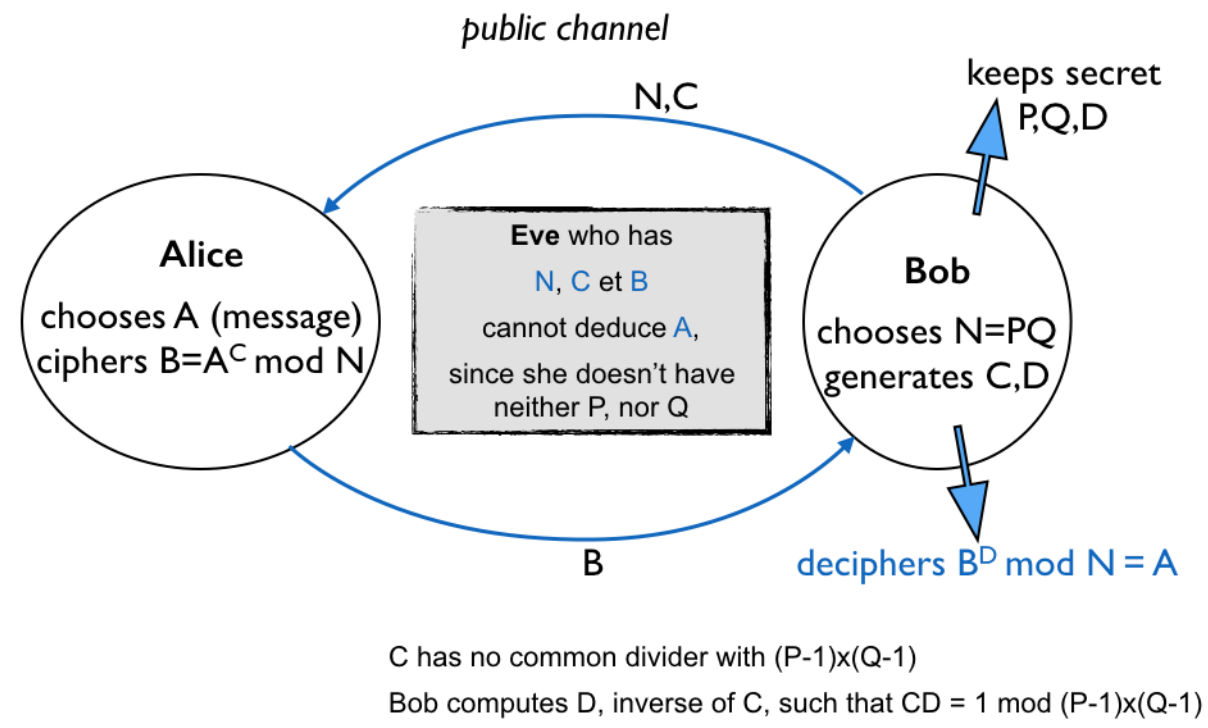

Figure 1.4: The schematic showing the principle of RSA protocol.

In public key cryptosystems (see Fig.1.4), two communicating parties each acquire two keys. One key is private and it is kept locally by one user, say Bob. Contrarily, the second one is public, and this particular key is made available for the rest of the world to see, say any potential Alice. In this case, the ciphering of secret messages is done via the use of the announced public key. Only the owner of such public key can decode the ciphered messages using his/her own private key. This can be achieved, if and only if, both private and public keys are somehow mathematically related.

The original idea behind the public-key cryptosystem comes from "Diffie-Hellman-Merkle key exchange" concept. However, it was a devised version, jointly developed by R. Rivest, A. Shamir and L. Adleman (RSA) in 1983 which has been extensively used until today. The basic operation of RSA works as follows (see also Fig.1.4):

1. Initially, Bob chooses randomly two large and and distinct prime integers $p$ and $q$.

2. Bob then computes $p \cdot q=n$ and $n$ is called the modulus.

3. He then chooses an integer $e$ with the condition that $e<n$ and $e$ is co-prime to $(p-1)(q-1)$.

4. Next, he computes another parameter $d$ for which $(d \cdot e-1)$ is divisible by $(p-1)(q-1)$. 
5. Eventually, $n$ and $e$ are announced by Bob as the public key. He keeps $d$ to himself and together with $n$, they act as the private key.

6. Alice or any potential user receive both $n$ and $e$. She then uses them to encrypt the message $m$. A ciphered message $c$ is derived from $c=m^{e} \bmod n$. The ciphered message $c$ is then sent to Bob.

7. Bob finally decrypts the ciphered message $c$ by performing $c^{d} \bmod n=m$.

Obviously, an eavesdropper Eve can intercept $n$ and can try to factorize the modulus $n$ in order to find $p$ and $q$ which leads to $d$. However, such an operation is extremely hard to compute, it requires heavy resources and is time consuming. For instance, consider two prime numbers $a=31$ and $b=51$. While calculating $a \times b=x=1829$ is relatively easy, deriving $a$ and $b$ from $x=1829$ is not something that we can do on-the-fly as it requires special algorithm. This is what considered to be the mathematical trap-door problem. Even though interesting, such type of security has never been proven. Today, it is still considered secure due to the lack of better factorization algorithm on top of slow performing computers. For what it's worth, the RSA cryptosystem is still widely used, for instance, by financial institutions and all over the Internet.

\section{Private key encryption: the OTP protocol}

When asked if there exists a conventional cryptography that is mathematically proven to be absolutely secure, it would be the Vernam's one-time pad (OTP) encryption protocol. OTP is proven to be uncrackable. It is immune even against cryptanalysis. In order to really understand the OTP protocol, we consider the following scenario between Alice and Bob:

1. Initially, Alice and Bob share a private random key $k \in\{0,1\}^{n}$, where $n$ is the key size. In OTP implementation, the key needs to be of the same size as the intended message.

2. A message $m \in\{0,1\}^{n}$ is prepared by Alice. Next, she performs the modulo-2 addition (XOR operation) between $k$ and $m$, resulting in a completely random encrypted message $e \rightarrow m \oplus k=e$. 
3. Alice sends $e$ to Bob.

4. Upon receiving $e$, Bob performs the same XOR operation between $k$ and $e$, resulting in $c \oplus k=m$.

The strength of this encryption method lies in the fact that the generated encrypted message is built up from a completely random sequence of bits $k$, showing hence zero redundancy nor correlation. However, the key itself needs to be generated in a completely random manner and can only be used once per message, hence the name "one-time pad". Yet secure, OTP is still considered inefficient. Similar to older cryptosystems, the big problem in clouding OTP implementation is that the key needs to be secretly shared by Alice and Bob. Furthermore, true randomness needs to be available at the key creation process for which such element is something that our macroscopic world will never provide.

It is the aim of this chapter to show that quantum physics can actually provide viable solutions to the problems discussed above. It is all summed up in the development of quantum key distribution (QKD) protocols. As a whole, QKD itself cannot be regarded as a complete cryptosystem, but it will act as the perfect complementary tool for the OTP technique and QKD can somehow be seen as "secret key provider" tool. Starting from the next section, we will explore, briefly, the world of quantum information, starting from the explanation of the qubit, which is the smallest unit of quantum information.

\subsection{Quantum communication}

Analogously to classical information, a smallest unit for quantum information can also be defined. Likewise, this quantum unit can also take either the value 0 and 1 . However, in the quantum world, the information can be treated differently, in a way that it will open the door into new possibilities and paradigms. So, what is all the fuss about?

\subsubsection{The qubit}

The counterpart of the bit is the qubit (quantum bit). In general, we define a qubit as a two-level quantum system. Such system exists in its own restricted, 2-dimensional complex vector space, i.e. Hilbert space. Here, a quantum state simply points to a position in that 
space for which the position can be further expressed in term of the basis vectors. This can be represented on the Bloch-Poincare sphere of Fig.1.5. Using the so-called Dirac notation, we can introduce the natural computation basis for a qubit which is $\mathcal{B}:\{|0\rangle,|1\rangle\}$, where $|0\rangle$ and $|1\rangle$ represent a pair of linearly independent vectors in a 2-dimensional Hilbert space.

What makes a qubit so special? Well, instead of being either only 0 or 1 like for classical bits, a qubit can exist in a state such as

$$
|\psi\rangle=\alpha|0\rangle+\beta|1\rangle
$$

The state $|\psi\rangle$ represents the general form of a qubit. As can be seen in Eq.(1.1), quantum theory defines a qubit as a coherent superposition of sates (in this case, these two states are labeled $|0\rangle$ and $|1\rangle)$. Already, this makes a qubit itself more general compared to a classical bit. Here, $\alpha$ and $\beta$ are complex numbers. As in common practice, $|\psi\rangle=\alpha|0\rangle+\beta|1\rangle$ can also be rewritten as

$$
|\psi\rangle=\left(\begin{array}{l}
\alpha \\
\beta
\end{array}\right),
$$

where

$$
|0\rangle=\left(\begin{array}{l}
1 \\
0
\end{array}\right),|1\rangle=\left(\begin{array}{l}
0 \\
1
\end{array}\right)
$$

Looking at such property of qubit $|\psi\rangle$, one can ask a very important question: how do we interpret the value of a qubit? Although it may seem paltry to some, for us to know what state the qubit $|\psi\rangle$ actually is in, a projective measurement has to be applied. Here, we stress that a quantum system can simultaneously be in different states before measurement. Having said that, the outcome of such measurement is actually simpler than one can imagine. In the simplest form, a measurement of the general state $|\psi\rangle=\alpha|0\rangle+\beta|1\rangle$ in the standard computational basis $\mathcal{B}:\{0,1\}$ yields, randomly, either $|0\rangle$ or $|1\rangle$ which can be associated with classical bits of 0 and 1 , respectively. This is because the probabilities of detection are given by the square modulus of the superposition coefficients $\alpha$ and $\beta$. In other words, $|\alpha|^{2}$ defines the probability of collapsing $|\psi\rangle$ in 0 and $|\beta|^{2}$ defines the probability of collapsing $|\psi\rangle$ in 1 . Therefore, in the quantum world, the following normalization condition 
should be satisfied,

$$
|\alpha|^{2}+|\beta|^{2}=1
$$

Ergo, the qubit state $|\psi\rangle$ can be represented by the following form

$$
|\psi\rangle=\cos \theta|0\rangle+e^{i \varphi} \sin \theta|1\rangle,
$$

where $\theta \in\left[0: \frac{\pi}{2}\right]$ and $\varphi \in[0: 2 \pi]$. So, it is possible to define a qubit state from those two parameters $\theta$ and $\varphi$, thus allowing such state to point at the surface of the Bloch-Poincaré sphere (see Fig.1.5). On the sphere, $|0\rangle$ represents the north pole while $|0\rangle$ depicts the south pole. Meanwhile, the other two complementary bases $\left\{\frac{1}{\sqrt{2}}(|0\rangle+|1\rangle), \frac{1}{\sqrt{2}}(|0\rangle-|1\rangle)\right\}$ and $\left\{\frac{1}{\sqrt{2}}(|0\rangle+i|1\rangle), \frac{1}{\sqrt{2}}(|0\rangle-i|1\rangle)\right\}$, located on the equator, represent the linear combination of the orthogonal basis $\{|0\rangle ;|1\rangle\}$.

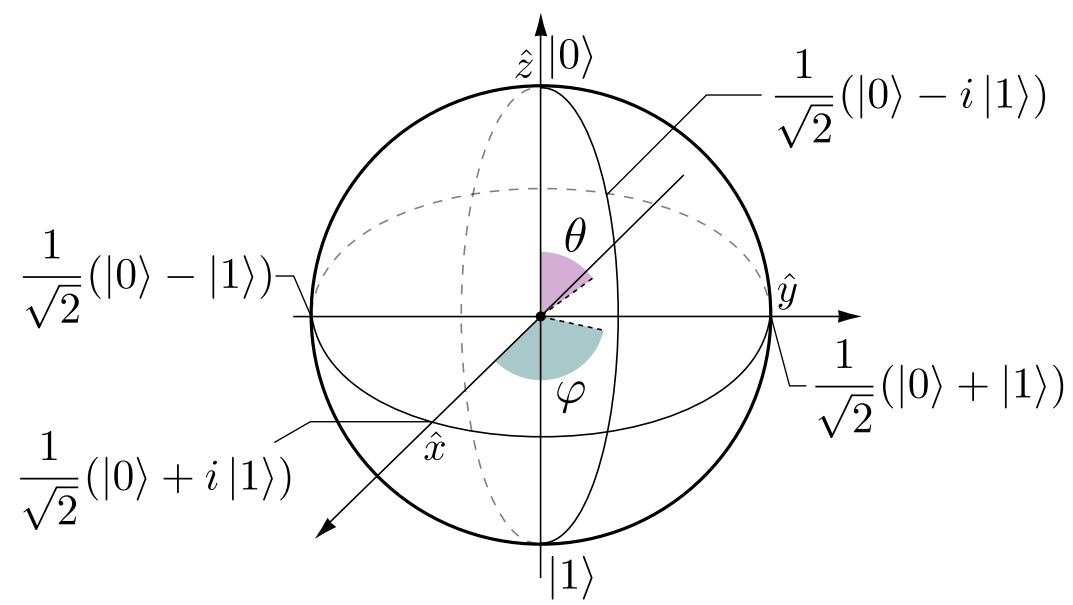

Figure 1.5: The Bloch-Poincaré sphere representation for the qubit $|\psi\rangle$ described in Eq.(1.5). The north and south poles are represented by the state $|0\rangle$ and $|1\rangle$, respectively. Meanwhile, the other four poles, located on the equator, depict the linear combination of the orthogonal basis vectors $\{|0\rangle ;|1\rangle\}$.

It is also important to note that a measurement perturbs the initial state of the qubit, meaning the state after the measurement is associated with the outcome of the measurement.

\subsubsection{A short note on complementary bases}

In a 2-dimensional Hilbert space, any pair of vectors that is linearly independent could form a basis. With this definition, we already defined the computational basis $\mathcal{B}:\{|0\rangle,|1\rangle\}$. The 
basis $\mathcal{B}$ is said to be orthonormal as the inner products of the vectors $\langle 0 \mid 0\rangle=\langle 1 \mid 1\rangle=1$ and $\langle 0 \mid 1\rangle=\langle 1 \mid 0\rangle=0$.

Conveniently, other orthonormal bases can also be introduced, for instance $\mathcal{B}^{\prime}$ : $\left\{\frac{|0\rangle+|1\rangle}{\sqrt{2}}, \frac{|0\rangle-|1\rangle}{\sqrt{2}}\right\}$. Now, let us perform a simple calculation involving two different vectors correspond each to $\mathcal{B}$ and $\mathcal{B}^{\prime}$,

$$
\mid\left\langle 0\left|\frac{|0\rangle+|1\rangle}{\sqrt{2}}\right|^{2}=\frac{1}{2}(\langle 0 \mid 0\rangle+\langle 0 \mid 1\rangle)=\frac{1}{2} .\right.
$$

The result obtained in Eq.(1.6) strongly suggests that both bases $\mathcal{B}$ and $\mathcal{B}^{\prime}$ are incompatible, i.e. they are complementary from the measurement point of view [Bengtsson 2007]. The above calculation can be interpreted as finding $\frac{1}{2}$ probability of obtaining the state $|0\rangle$ when the qubit $\frac{|0\rangle+|1\rangle}{\sqrt{2}}$ is projected on the basis state $|0\rangle$. As a side note, the outer product $|0\rangle\langle 0|$ represents the projection operator on the state $|0\rangle$.

Alternatively, we explain the concept of complementary bases as follows: we consider two parties Alice and Bob. In her lab, Alice prepares a large number of qubits, solely in the basis $\mathcal{B}:\{|0\rangle,|1\rangle\}$. She then sends all the prepared qubits to Bob. Bob, on the other hand, is completely clueless about the preparation basis used by Alice. Within his capability, he has only two choices for the measurement basis. If he decides to measure the qubits in the $\mathcal{B}$ basis, he will then obtain deterministic results with unit probability. Otherwise, with basis $\mathcal{B}^{\prime}:\left\{\frac{|0\rangle+|1\rangle}{\sqrt{2}}, \frac{|0\rangle-|1\rangle}{\sqrt{2}}\right\}$, his measurements will yield probabilistic outcomes with the probability of $\frac{1}{2}$.

An example of probabilistic measurement concerning polarized photons is depicted in Fig.1.6. We will see later in Sec.1.2.5 how the concept of probabilistic measurement plays a major role in quantum key distribution (QKD) protocols.

\subsubsection{Cloning a qubit}

It is obvious that due to quantum superposition, a performed measurement will perturb a quantum system, thus our knowledge on an unknown $|\psi\rangle$ is limited. Could we really use this kind of incomplete information, for example, to regenerate the unknown state of $|\psi\rangle$ ? In other words, is it possible to perform a perfect cloning of $|\psi\rangle$ ?

Imagine a perfect quantum cloning machine depicted in Fig.1.7 for which the working 
a)

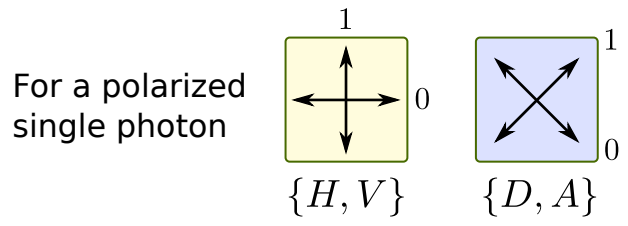

b)

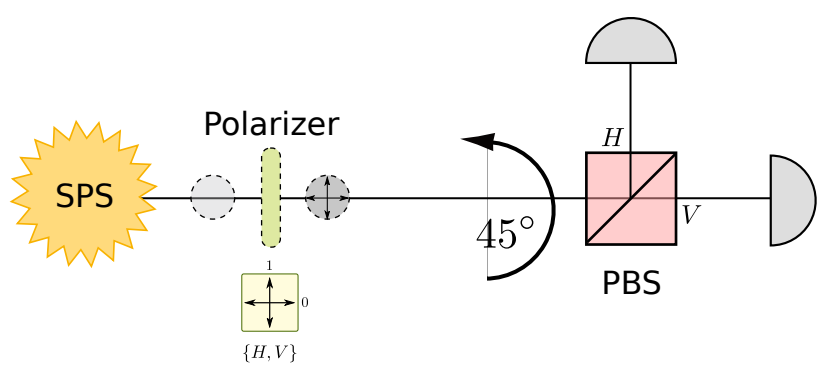

Figure 1.6: (a): An example of two complementary bases for polarized single photons. The polarization states are defined as H: horizontal, V: vertical, D: diagonal, A: anti-diagonal. 0 and 1 are the associated logic bit values. (b): A single photon produced by a single photon source (SPS) is prepared in the $\{H, V\}$ basis with the help of a polarizer. The photon is then analyzed by employing a polarizing beam splitter (PBS) and two single photon detectors. If the PBS has the same orientation as that of the creation basis, the outcomes are deterministic. If the PBS is rotated by $45^{\circ}$, the creation and analysis bases are complimentary, thus resulting in probabilistic outcomes with 50/50 chances for the single photon to be either detected by the upper or the lower detector.

principle can be modeled as

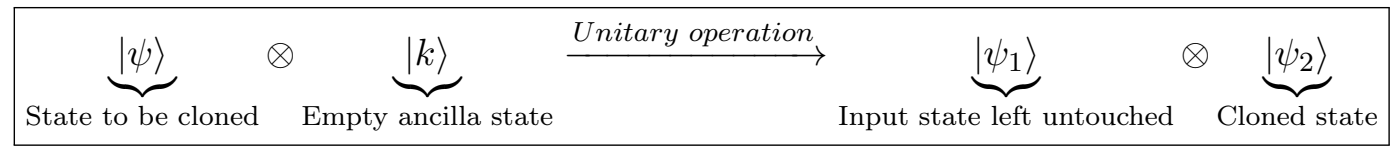

where $\left|\psi_{1}\right\rangle=\left|\psi_{2}\right\rangle=|\psi\rangle$. Here, the ultimate goal is to reproduce the state $|\psi\rangle$ of an unknown qubit on an empty ancillary qubit $|k\rangle$. If the perfect cloning operation is successfully performed, two states $|\psi\rangle_{1}$ and $|\psi\rangle_{2}$ will be produced as perfect replica of the input state $|\psi\rangle$. In general, an "ideal" QCM should feature three major characteristics:

- Universality: the quantum cloning machine should work identically regardless of the input state.

- Optimum fidelity: it is the overlap between the output states and the input state, $\mathrm{F}_{i}=\left\langle\psi\left|\psi_{i}\right| \psi\right\rangle$. Ideally, $\mathrm{F}_{i} \rightarrow 1$.

- Symmetry: it defines the indistinguishability between the two output states. 


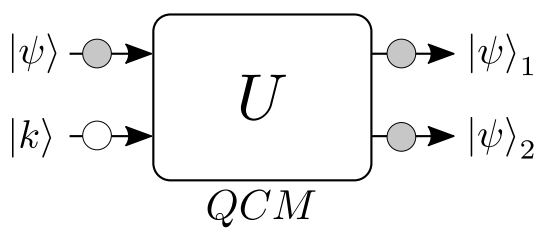

Figure 1.7: Representation of a quantum cloning machine (QCM). The system is fed with two inputs, e.g. two photonic qubits. Here, we aim to clone the state $|\psi\rangle$ of the gray photon to an ancillary blank photon which is initially prepared in the state $|k\rangle$. Once the machine performs the unitary transformation $U$, two gray photons in the state $|\psi\rangle_{1}$ and $|\psi\rangle_{2}$ are made available at the output, with $|\psi\rangle=|\psi\rangle_{1}=|\psi\rangle_{2}$. It is important to note that the initial gray photon is not destroyed by the cloning operation.

Suppose that a unitary transformation $U$ (energy conserving and universal) can actually permit a perfect cloning of an unknown state $|\psi\rangle$ on an empty ancilla state $|k\rangle$, i.e.,

$$
U(|\psi\rangle|k\rangle)=|\psi\rangle|\psi\rangle
$$

In the quantum world, the state $|\psi\rangle$ can always be associated with a linear combination of orthogonal basis $\{|0\rangle ;|1\rangle\}$, leading to

$$
|\psi\rangle=\alpha|0\rangle+\beta|1\rangle
$$

Due to the universality of a perfect QCM, the unitary transformation $U$ should allow perfect cloning regardless of the input state. Therefore, $U$ should also be effective for cloning a general qubit state of the form $\alpha|0\rangle+\beta|1\rangle$. Due to the linearity of quantum physics,

$$
\begin{aligned}
U[(\alpha|0\rangle+\beta|1\rangle)|k\rangle] & =\alpha U(|0\rangle|k\rangle)+\beta U(|1\rangle|k\rangle) \\
& =\alpha|0\rangle|0\rangle+\beta|1\rangle|1\rangle .
\end{aligned}
$$

But this is in full contradiction to the fact that perfect cloning produces two identical outputs,

$$
\begin{aligned}
U[(\alpha|0\rangle+\beta|1\rangle)|k\rangle] & =(\alpha|0\rangle+\beta|1\rangle)(\alpha|0\rangle+\beta|1\rangle) \\
& =\alpha^{2}|0\rangle|0\rangle+\beta^{2}|1\rangle|1\rangle+\alpha \beta(|0\rangle|1\rangle+|1\rangle|0\rangle) .
\end{aligned}
$$


The difference in the results of Eq.(1.9) and Eq.(1.10) clearly states that a perfect cloning of an unknown quantum state $|\psi\rangle$ is not possible. This affair has been long discussed, and it was Wigner who was probably the first to emphasized the problem of selfcloning in quantum formalism, and the theory was later completed by Wootters and Zurek [Wootters \& Zurek 1982]. However, the impossibility of cloning an unknown quantum state never stopped researchers from proposing universal, symmetry quantum cloning machines. A pertinent and important of such machines has been reported by Bužek and Hillery, finding the optimum fidelity $\mathrm{F}$ of $\frac{5}{6} \simeq 83 \%$, and has been demonstrated experimentally via stimulated emission in non-linear crystals [Lamas-Linares et al. 2002] and in optical fiber amplifier as gain media [Fasel et al. 2002].

At a glance, such restriction imposed by quantum superposition is deemed troublesome, but the underlying advantage it brings may change the way we communicate.

\subsubsection{Entanglement in bipartite quantum systems}

A quantum system can also be composed of $n$-qubit systems, where each qubit can be defined in the computational basis $B=\{|0\rangle,|1\rangle\}$. For instance, a two-qubit system $a$ and $b$, also said bipartite system, comprises two 2-dimensional Hilbert spaces, $\mathcal{H}_{a}$ and $\mathcal{H}_{b} d \mathcal{H}_{a, b}=2$. Consequently, the Hilbert space, and its dimension, in which the bipartite system is defined are given by

$$
\mathcal{H}=\mathcal{H}_{a} \otimes \mathcal{H}_{b} \text { with } d \mathcal{H}=d \mathcal{H}_{a} \times d \mathcal{H}_{b}=4 .
$$

Therefore, the possible states of a bipartite qubit system in the computational basis are $|0\rangle_{a}|0\rangle_{b},|0\rangle_{a}|1\rangle_{b},|1\rangle_{a}|0\rangle_{b}$, and $|1\rangle_{a}|1\rangle_{b}$. Here, the coherent superposition of states governed by quantum physics is always applicable, thus a general bipartite system $|\psi\rangle_{a b}$ can be written as

$$
|\psi\rangle_{a b}=\alpha_{a b}|0\rangle_{a}|0\rangle_{b}+\beta_{a b}|0\rangle_{a}|1\rangle_{b}+\gamma_{a b}|1\rangle_{a}|0\rangle_{b}+\rho_{a b}|1\rangle_{a}|1\rangle_{b}
$$

with the normalization condition

$$
\left|\alpha_{a b}\right|^{2}+\left|\beta_{a b}\right|^{2}+\left|\gamma_{a b}\right|^{2}+\left|\rho_{a b}\right|^{2}=1
$$


In some cases, depending on the given superposition coefficients, the state $|\psi\rangle_{a b}$ can further be separated into two sub-states $|\psi\rangle_{a} \otimes|\psi\rangle_{b}$, which represents two independent quantum subsystems. Such a situation can be obtained, for instance, with $\alpha_{a b}=\beta_{a b}=$ $\gamma_{a b}=\rho_{a b}=\frac{1}{2}$,

$$
\begin{aligned}
|\psi\rangle_{a b} & =\frac{1}{2}\left(|0\rangle_{a}|0\rangle_{b}+|0\rangle_{a}|1\rangle_{b}+|1\rangle_{a}|0\rangle_{b}+|1\rangle_{a}|1\rangle_{b}\right) \\
& =\frac{1}{2}\left(|0\rangle_{a}+|1\rangle_{a}\right)\left(|0\rangle_{b}+|1\rangle_{b}\right)=\frac{1}{\sqrt{2}}\left(|0\rangle_{a}+|1\rangle_{a}\right) \frac{1}{\sqrt{2}}\left(|0\rangle_{b}+|1\rangle_{b}\right) \\
& =|\psi\rangle_{a} \otimes|\psi\rangle_{b} .
\end{aligned}
$$

In this case, $|\psi\rangle_{a} \otimes|\psi\rangle_{b}$ represents a product state, for which any measurement on quantum subsystem $a$ will not affect quantum subsystem $b$, and conversely.

However, there are also cases where the separation of a quantum system into two subsystems is not possible, $|\psi\rangle_{a b} \neq|\psi\rangle_{a} \otimes|\psi\rangle_{b}$. Here, the whole state $|\psi\rangle$ is "perfectly" defined but the individuals are not, i.e. $a$ and $b$ cannot belong to a single quantum system. We consider the following inseparable quantum state of the form

$$
|\psi\rangle_{a b}=\frac{1}{\sqrt{2}}\left(|0\rangle_{a}|0\rangle_{b}+|1\rangle_{a}|1\rangle_{b}\right)
$$

$|\psi\rangle_{a b}$ is called to be an entangled state. Any measurement on the quantum subsystem $a$ randomly reveals either $|0\rangle$ or $|1\rangle$, but at the same time, it imposes the state that belongs to the quantum subsystem $b$. In general, this inseparability is a specific property of a composed quantum system that can be formed from one, two or more subsystems, and this is what is known as quantum entanglement.

In a bipartite system, there are four particularly famous inseparable states,

$$
\left\{\begin{aligned}
\left|\Psi^{+}\right\rangle_{a b} & =\frac{1}{\sqrt{2}}\left(|0\rangle_{a}|1\rangle_{b}+|1\rangle_{a}|0\rangle_{b}\right), \\
\left|\Psi^{-}\right\rangle_{a b} & =\frac{1}{\sqrt{2}}\left(|0\rangle_{a}|1\rangle_{b}-|1\rangle_{a}|0\rangle_{b}\right), \\
\left|\Phi^{+}\right\rangle_{a b} & =\frac{1}{\sqrt{2}}\left(|0\rangle_{a}|0\rangle_{b}+|1\rangle_{a}|1\rangle_{b}\right) \\
\left|\Phi^{-}\right\rangle_{a b} & =\frac{1}{\sqrt{2}}\left(|0\rangle_{a}|0\rangle_{b}-|1\rangle_{a}|1\rangle_{b}\right)
\end{aligned}\right.
$$

where all the states are considered maximally entangled due to equally-weighted normalization. These four states are called the Bell states. 


\subsubsection{A case to ponder}

Alice

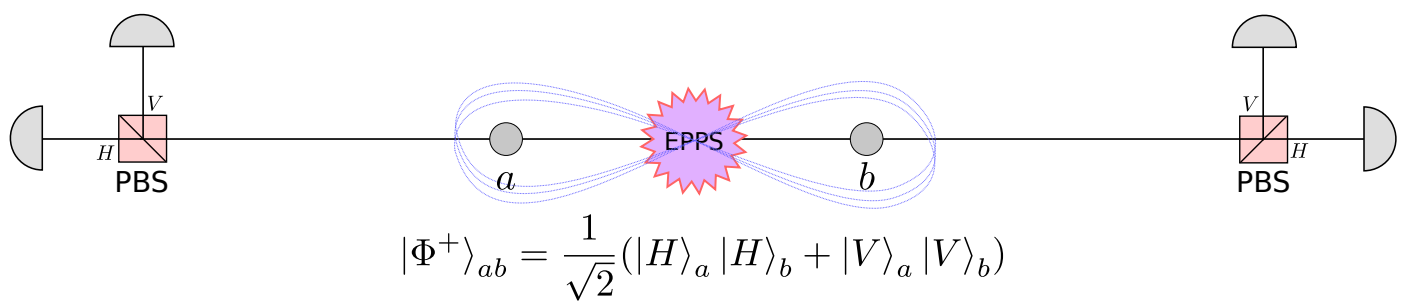

Figure 1.8: Alice and Bob share a quantum resource which is an entangled photon pair source (EPPS). The EPPS emits photon pairs in the entangled state $\left|\Phi^{+}\right\rangle_{a b}=\frac{1}{\sqrt{2}}\left(|0\rangle_{a}|0\rangle_{b}+\right.$ $\left.|1\rangle_{a}|1\rangle_{b}\right)$. Photons from the same pair will travel in opposite directions, one goes to Alice and the other one goes to Bob. Equipped with polarization measurement basis consists of a polarizing beam splitter (PBS) and two detectors, both parties will record which detector fires upon the arrival of each photons.

We consider a situation where an entangled photon pair source (EPPS) emits photon pairs in the maximally entangled state $\left|\Phi^{+}\right\rangle_{a b}=\frac{1}{\sqrt{2}}\left(|H\rangle_{a}|H\rangle_{b}+|V\rangle_{a}|V\rangle_{b}\right)$ (see Fig.1.8). Here, photons from the same pair are separated and travel in different directions, one goes to Alice and the other one goes to Bob. Upon receiving their respective photon, Alice and Bob both perform a measurement in $\{|H\rangle,|V\rangle\}$ basis. Let us suppose that Alice knows beforehand that the source is indeed an entanglement source and the second photon $b$ will end up at a trusted party Bob. Once Alice measures her qubit to be $|H\rangle$, she will know for sure that Bob's qubit will also collapse onto $|H\rangle$, even before Bob performs his measurement. The measurement outcomes are noted and Alice will eventually compare her results to that of Bob. Quantum physics predicts that there will be strong correlations between both measurement outcomes and this is exactly what Alice observes. The only thing that the quantum physics cannot predict is the state of Alice's (or Bob') photon before the measurement.

A second set of measurements is conducted, but this time the source is replaced by another source $S^{\dagger}$. The very same procedures will be conducted, but with the following conditions:

- Rather than entangled states, the source $S^{\dagger}$ produces a mere mixture of states made of $\left|\Phi^{2}\right\rangle_{a b}=|0\rangle_{a}|0\rangle_{b}$ and $\left|\Phi^{\imath}\right\rangle_{a b}=|1\rangle_{a}|1\rangle_{b}$, with $P_{\left|\Phi^{2}\right\rangle_{a b}}=P_{\left|\Phi^{u \prime}\right\rangle_{a b}}=\frac{1}{2}$. 
- The source $S^{\dagger}$ is kept secret to Alice.

Surprisingly, the new outcomes still show strong correlations as before. Therefore, Alice might think that the source $S^{\dagger}$ is indeed an entanglement source. Is she really capable of differentiating between the two type of sources? With current procedures, she can never tell. The truth is, the perfect correlation observed with the source $S^{\dagger}$ producing a mixture of states is only due deterministic outcomes as the analysis basis is the same as the preparation basis (see also Fig.1.6). So in this case, the simple trick for them would be to perform their measurements in the complementary basis and they will no longer observe the perfect correlation. In other words, with the EPPS, they will observe strong (quantum) correlation, while with $S^{\dagger}$, no correlation persist in the complementary basis. The correlation induced by entanglement is indeed invariant by rotation of the analyzers. This property is the key to actually verify and quantify quantum entanglement, and more importantly, it provides the answers to what is known today as the EPR paradox.

\subsubsection{The EPR paradox}

The Einstein-Podolsky-Rosen (EPR) paradox originally discussed in 1935 basically represented the most important challenge from Albert Einstein to the quantum community [Einstein et al. 1935]. The fact that quantum theory works really well was of no discussion to Einstein. All he ever wanted is the truth. For him, nothing could guarantee the truth even if a theory works for centuries (remember Newton's gravitation theory and special relativity discovery?). The very nature of quantum theory itself proved to be troublesome for Einstein. Worst, he just simply could not accept the idea of not having pre-determined momentum and position values for entangled quantum particles which is the essence behind the Heisenberg uncertainty principle. Even so, he never claimed that the quantum theory championed by Niels Bohr was wrong, but rather incomplete. Basically, through this challenge, he tried to explain the quantum physics by introducing local hidden variable theory (LHVT).

For now, we will continue on the previous experiment (see Fig.1.8). It represents an ideal scenario for discussing the EPR paradox as it provides the most crucial ingredient which is entanglement. We know that if Alice measures her photon, she will gain the 
information on Bob's state without he even needs to measure his photon. Specifically, if Alice's measurement reveals $|H\rangle$, so will Bob. This is what is predicted by quantum mechanics. As for Einstein, he predicted the same outcome. So where is the paradox?

EPR paradox, in layman's terms: Alice and Bob both share a pair of entangled photons whose quantum state reads $\left|\Phi^{+}\right\rangle_{a b}=\frac{1}{\sqrt{2}}\left(|H\rangle_{a}|H\rangle_{b}+|V\rangle_{a}|V\rangle_{b}\right)$. Say Alice measures her photon in the $\{|H\rangle,|V\rangle\}$ basis. If Alice's measurement reveals $|H\rangle$, she can deduce the outcome of Bob's measurement to be $|H\rangle$, provided Bob uses compatible basis. To her surprise, Bob decides to go with the complementary basis $\{|D\rangle,|A\rangle\}$ instead. Let us assume Bob finds $|D\rangle$ as the outcome. At the same time, he knows that from Alice's measurement, should he used the same basis as Alice, i.e. $\{|H\rangle,|V\rangle\}$, he would got $|H\rangle$ instead. Therefore, Bob now has information on outcomes from two complementary bases. However, according to Heisenberg's uncertainty principle, this is just plain wrong. But how these two photons know what is going on on the other side of the experiment? Generally, people come up with two explanations, either these two photons possibly "talk" to each other in superluminal fashion, or, there is pre-existing information embedded on these two photons when they were first simultaneously generated at the source. Due to special relativity, Einstein champions the latter hence the introduction of the local hidden variable models. Nonetheless, it seems that people forget that those two photons were actually entangled to begin with. When Alice performed her measurement, the entangled state had already collapsed. Surely after that, Bob can measure his respective photon in complementary basis, but what he will ever get is a meaningless, truly randomized outcome.

Since its appearance, the EPR paradox had been under serious debates between scientists for about 30 years. As for Niels Bohr, he stood firm with his opinion that the measurement outcomes are not pre-determined as well as the single qubit states are composing an entangled pair of qubits. For Bohr's and his supporters, an entangled state, as a whole, is "perfectly" defined but the individuals are not. As in 1964, there came Bell that took Einstein's arguments very seriously and proposed a way to verify the existence, or not, of these so-called local hidden variables. 


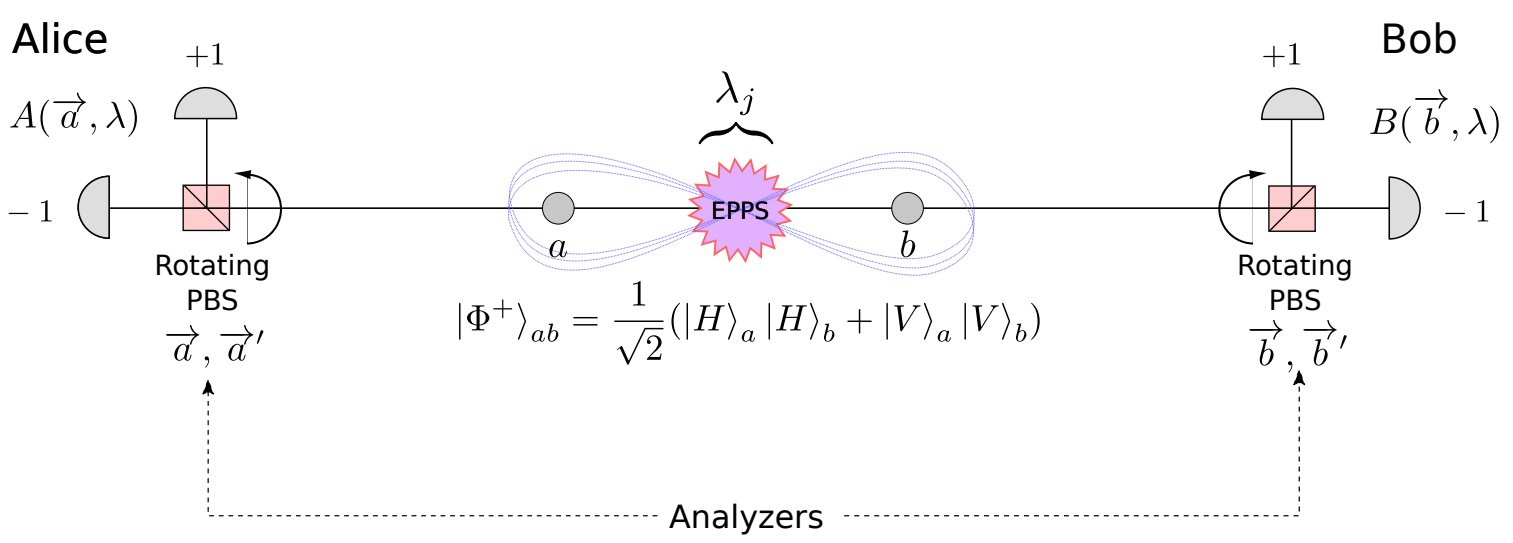

Figure 1.9: The experimental setup for the violation of Bell-CHSH inequality. It is more or less the same with the experiment discussed in Sec.1.2.3.1. The only noticeable difference is the introduction of rotating polarizing beam splitters (PBS) as polarization-state analyzers.

\subsubsection{The Bell-CHSH inequality}

Originally, Bell simply suggested to define a specific boundary on the statistical results of space-separated measurements which can be used to differentiate between the quantum and the classical worlds. Considering Bohr's opinion that the measurement outcomes cannot not be pre-determined, random measurement settings are applied. Thus, different measurement outcomes are expected. The outcomes for repeated measurements are recorded and the corresponding statistics is computed. If the statistics violates the Bell's inequality, local hidden variable (LHV) theories are then simply out of the picture.

To further describe the idea of Bell, again, we can always resort to the previous experiment discussed in section 1.2.3.1. An updated version of such experiment is depicted in Fig.1.9 where the use of entangled photon pair source producing the maximally entangled state

$$
\left|\Phi^{+}\right\rangle_{a b}=\frac{1}{\sqrt{2}}\left(|H\rangle_{a}|H\rangle_{b}+|V\rangle_{a}|V\rangle_{b}\right)
$$

is maintained.

First, let us consider Einstein's arguments of the LHV theory. For this, we define $\lambda$ as the set of local hidden variables manifested in each $j^{\text {th }}$ generated photon pairs. Photons from the same pair then travel in opposite direction toward two different measurement apparatuses belonging to Alice and Bob, respectively. Alice and Bob are space-separated so as to comply with special relativity. This way, any outcome on Alice's measurements is 
independent on Bob's, and conversely, which ensures the condition of locality.

Alice and Bob both employ an analyzer followed by two detectors so as to perform a linear polarization measurement on each incoming photons with a dichotomous outcome spectrum of \pm 1 . As the quantum system is supposedly governed by the LHV model, detector in which the photon ends up not only depends on the adjustable analyzer parameters $\vec{a}$ and $\vec{a}^{\prime}$ at Alice's, $\vec{b}$ and $\vec{b}^{\prime}$ at Bob's, but also on the the local hidden variable $\lambda$. As a matter of fact, $\vec{a}, \vec{a}^{\prime}$ and $\vec{b}, \vec{b}^{\prime}$ are two possible orientations of the analyzers at Alice's and Bob's locations, respectively. Consequently, the possible outcomes for each detected photon by both parties can be represented by the following deterministic functions

$$
\begin{gathered}
A(\vec{a}, \lambda)=A, \\
A\left(\vec{a}^{\prime}, \lambda\right)=A^{\prime}, \\
B(\vec{b}, \lambda)=B, \\
B\left(\vec{b}^{\prime}, \lambda\right)=B^{\prime} .
\end{gathered}
$$

As per Bell's theorem, we then consider then the following quantity $S$,

$$
S=A B+A B^{\prime}-A^{\prime} B+A^{\prime} B^{\prime}=\left(A-A^{\prime}\right) B+\left(A+A^{\prime}\right) B^{\prime} .
$$

By taking into account Einstein's LHV theory, we can straightforwardly deduce the limits of the quantity $S$ for $A, A^{\prime}, B, B^{\prime}= \pm 1$,

$$
-2 \leq S \leq+2
$$

which can be further expressed as

$$
-2 \leq E(\vec{a}, \vec{b})+E\left(\vec{a}^{\prime}, \vec{b}\right)+E\left(\vec{a}^{\prime}, \vec{b}^{\prime}\right)-E\left(\vec{a}, \vec{b}^{\prime}\right) \leq+2
$$

where $E$ is the expectation values for each combination of orientations.

Eq.(1.24) simply refers to a derived version of Bell's inequality proposed by Clauser, Horne, Shimony and Holt, i.e. Bell-CHSH inequality [Clauser et al. 1969] which is better adapted to real experiments. Here, the quantity $S$ is famously known as the Bell parameter 
which can be used to quantify the level of entanglement contained in a particular entangled state.

From now on, we are going to investigate whether or not quantum physics is in agreement with Einstein's LHV model. As per Bell's theorem requirement, two measurement settings for each adjustable analyzer parameters $\vec{a}$ and $\vec{b}$ are fixed. The new corresponding bases for $\vec{a}$ and $\vec{b}$ will be $\left\{|\varphi\rangle_{a},|\varphi\rangle_{a}^{\prime}\right\}$ and $\left\{|\varphi\rangle_{b},|\varphi\rangle_{b}^{\prime}\right\}$, respectively, where the states $|\varphi\rangle$ and $|\varphi\rangle^{\prime}$ point at relative angles $\varphi$ and $\varphi^{\prime}$ to the horizontal axis, respectively.

We actually can rewrite the individual state of a photon with unknown polarization in the new defined bases of $\left\{|\varphi\rangle_{a},|\varphi\rangle_{a}^{\prime}\right\}$ and $\left\{|\varphi\rangle_{b},|\varphi\rangle_{b}^{\prime}\right\}$. This leads to

$$
\begin{aligned}
|H\rangle_{a} & =\cos \varphi_{a}|\varphi\rangle_{a}-\sin \varphi_{a}|\varphi\rangle_{a}^{\prime} \\
|V\rangle_{a} & =\sin \varphi_{a}|\varphi\rangle_{a}+\cos \varphi_{a}|\varphi\rangle_{a}^{\prime} \\
|H\rangle_{b} & =\cos \varphi_{b}|\varphi\rangle_{b}-\sin \varphi_{b}|\varphi\rangle_{b}^{\prime} \\
|V\rangle_{b} & =\sin \varphi_{b}|\varphi\rangle_{b}+\cos \varphi_{b}|\varphi\rangle_{b}^{\prime}
\end{aligned}
$$

Straightforwardly, the maximally entangled state of Eq.(1.17) can be rewritten as

$$
\begin{aligned}
\left|\Phi^{+}\right\rangle_{a b}= & \frac{1}{\sqrt{2}}\left(\cos \left(\varphi_{a}-\varphi_{b}\right)\left|\varphi_{a}\right\rangle\left|\varphi_{b}\right\rangle+\sin \left(\varphi_{a}-\varphi_{b}\right)\left|\varphi_{a}\right\rangle\left|\varphi_{b}\right\rangle^{\prime}\right. \\
& \left.-\sin \left(\varphi_{a}-\varphi_{b}\right)\left|\varphi_{a}\right\rangle^{\prime}\left|\varphi_{b}\right\rangle \cos \left(\varphi_{a}-\varphi_{b}\right)\left|\varphi_{a}\right\rangle^{\prime}\left|\varphi_{b}\right\rangle^{\prime}\right)
\end{aligned}
$$

To actually compute the corresponding $S$ parameter, we need first to define the probabilities for the different possible measurement outcomes,

$$
\begin{gathered}
P_{a b}^{++}=\left|\left\langle\varphi_{a} \varphi_{b} \mid \Phi_{a b}^{+}\right\rangle\right|^{2}=\frac{1}{2} \cos ^{2}\left(\varphi_{a}-\varphi_{b}\right), \\
P_{a b}^{+-}=\left|\left\langle\varphi_{a} \varphi_{b^{\prime}} \mid \Phi_{a b}^{+}\right\rangle\right|^{2}=\frac{1}{2} \sin ^{2}\left(\varphi_{a}-\varphi_{b}\right), \\
P_{a b}^{-+}=\left|\left\langle\varphi_{a^{\prime}} \varphi_{b} \mid \Phi_{a b}^{+}\right\rangle\right|^{2}=\frac{1}{2} \sin ^{2}\left(\varphi_{a}-\varphi_{b}\right), \\
P_{a b}^{--}=\left|\left\langle\varphi_{a^{\prime}} \varphi_{b^{\prime}} \mid \Phi_{a b}^{+}\right\rangle\right|^{2}=\frac{1}{2} \cos ^{2}\left(\varphi_{a}-\varphi_{b}\right) .
\end{gathered}
$$

Here, $P_{a b}^{++}$and $P_{a b}^{--}$are the probabilities of having both photons exit through the same output on both sides of the experiment. Meanwhile, the probabilities for both photons to 
exit through different outputs are represented by $P_{a b}^{+-}$and $P_{a b}^{-+}$. With these probabilities, we can calculate the expectation values $E_{a b}$ ( $\sum$ correlations $-\sum$ anticorrelations) that compose the Bell parameter $S$ (see Eq.(1.23)) by taking into account all the measurement probabilities. For example, we have

$$
\begin{aligned}
E_{a b} & =P_{a b}^{++}+P_{a b}^{--}-P_{a b}^{+-}-P_{a b}^{-+} \\
& =\cos ^{2}\left(\varphi_{a}-\varphi_{b}\right)-\sin ^{2}\left(\varphi_{a}-\varphi_{b}\right) \\
& =\cos \left(2\left(\varphi_{a}-\varphi_{b}\right)\right)
\end{aligned}
$$

Eventually, the $S$ parameter can be computed by summing up four expectation values where each corresponds to four different analyzer settings, as required for the violation of Bell's inequality,

$$
\begin{aligned}
S_{Q M} & =E(\vec{a}, \vec{b})+E\left(\vec{a}^{\prime}, \vec{b}\right)+E\left(\vec{a}^{\prime}, \vec{b}^{\prime}\right)-E\left(\vec{a}, \vec{b}^{\prime}\right) \\
& =\cos \left(2(\underbrace{\varphi_{a}-\varphi_{b}}_{\Theta})\right)+\cos \left(2(\underbrace{\varphi_{a}-\varphi_{b}^{\prime}}_{\Theta})\right)+\cos \left(2(\underbrace{\varphi_{a}^{\prime}-\varphi_{b}}_{\Theta})\right)-\cos \left(2(\underbrace{\varphi_{a}^{\prime}-\varphi_{b}^{\prime}}_{3 \Theta})\right) \\
& =\cos (2 \Theta)-\cos (6 \Theta),
\end{aligned}
$$

where $\Theta$ is the angel difference between analyzer settings. In Fig.1.10, we can see that the maximum violation of Bell's inequality is found at $\Theta=\frac{\pi}{8}$. This leads to

$$
S_{Q M}=3 \cos \left(\frac{2 \pi}{8}\right)-\cos \left(\frac{6 \pi}{8}\right)=\frac{3}{\sqrt{2}}+\frac{1}{\sqrt{2}}=2 \sqrt{2}
$$

and obviously, $S_{Q M}=2 \sqrt{2}>2$. As a consequence, quantum physics predictions clearly violate the Bell's inequality. In other words, this means that the concept of local hidden variables, i.e., pre-existing information, simply does not exist in the quantum world. Beyond that, later in Chapters 4 and 5 , the same condition of $S_{Q M}=2 \sqrt{2}>2$ will be demonstrated with our own polarization entangled photon pair sources.

Since Bell's theorem was introduced in 1964, the quest for experimental violations of his inequality has been long and hard. It actually started in 1972 when Freedman and Clauser tried for the first time to violate the Bell's inequality using polarization entangled photons emitted in an atomic cascade of calcium [Freedman \& Clauser 1972]. With their 


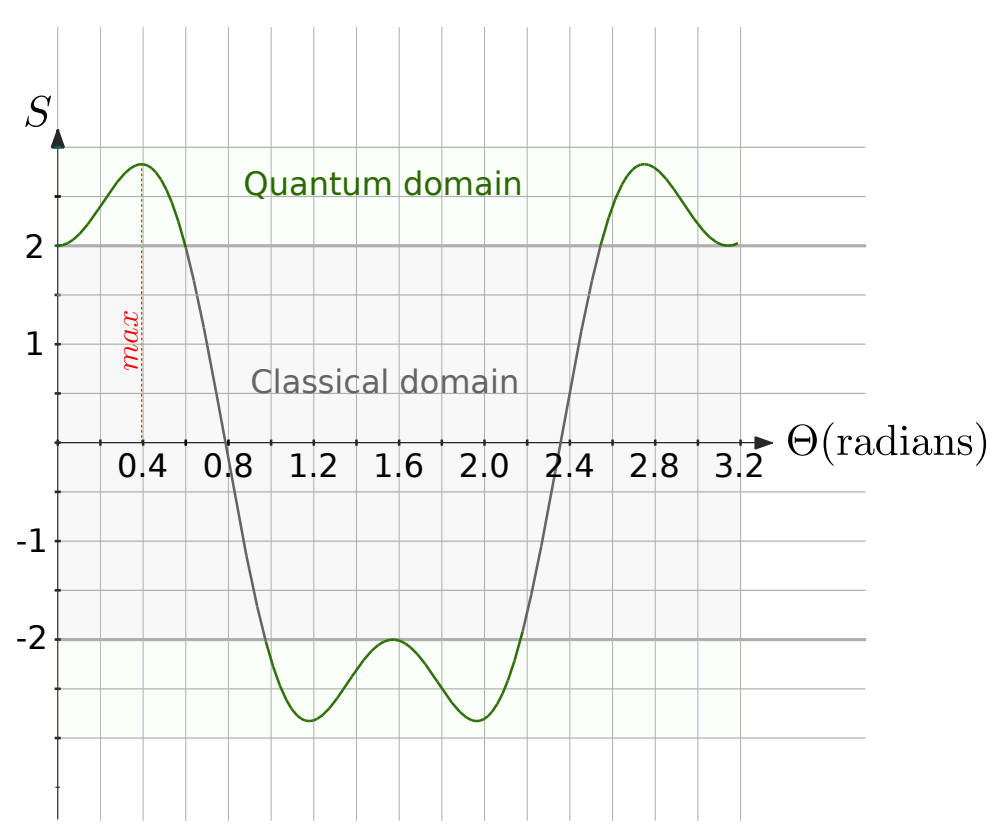

Figure 1.10: Evolution of the $S$ parameter as a function of $\Theta$ where the the boundaries between the classical and the quantum domains are clearly visible. The maximum violation of Bell's inequality is observed for $\Theta=\frac{\pi}{8}$, leading to $S_{Q M_{\max }}=2 \sqrt{2}$.

experiment, they could see a violation of the Bell's inequality. This first trial has been followed by several other attempts, violating or not, quantum theory. The uncertainty on the results was highly affected by these experimental constraints and difficulties. Then came in 1982 the "Orsay experiments" carried out by Aspect and his collaborators which were deemed to be the most successful attempt in violating Bell's inequality during that era [Aspect et al. 1981,Aspect et al. 1982a]. However, the quest was not over since loopholes, introduced by quantum physics non-believers, entered the game. In short, the two most wellknown loopholes are the locality [Bell 1964] and the detection [Pearle 1970] loopholes. Since then, we needed to wait thirty more years until a loophole-free Bell test was experimentally demonstrated by a group of researchers at Technical University of Delft [Hensen et al. 2015]. Alternatively, entanglement has become a key resource for quantum communication and processing, notably within the perspective of building device independent quantum systems. As a side note, information contained in [Aktas et al. 2015] might be of interest to some readers.

We will see in the next chapters of this manuscript how to exploit photonic entanglement for implementing long distance quantum networks operating at telecom wavelengths. 


\subsubsection{Photonic qubits}

Quantum superposition and entanglement are indeed mind boggling, so weird that nothing in our macroscopic world can imitate such behaviors. It is only when we zoom deeper to the extent of manipulating quantum systems that we can observe such intriguing phenomena.

Over the past few decades, we have seen tremendous progresses in the domain of photonics. From the first demonstration of Colladon's water jet to the actual use of guided waves in optical fibers for ultra-fast long distance networking, photonics is, together with electronics, somewhat we are best at. We are advancing to the extent where the generation, manipulation and detection of light all the way down to the single photon level are accessible. Together with enabling photonic technologies, photons are without doubt representing the quanta that we are most comfortable with.

In quantum communication, we are dealing with transferring qubits from one location to another over long distances. For this, nothing is better than the use of photons as information carrier. They are fast and can be emitted at wavelengths compatible with current deployed telecom infrastructures. Furthermore, they feature many observables that are suitable for quantum information encoding, being more or less robust against decoherence effects in optical fiber links. In the next section, we are going to explore the three most employed qubit encoding strategies in quantum communication.

\subsubsection{Qubit encoding strategies for quantum communication}

\section{Polarization encoding}

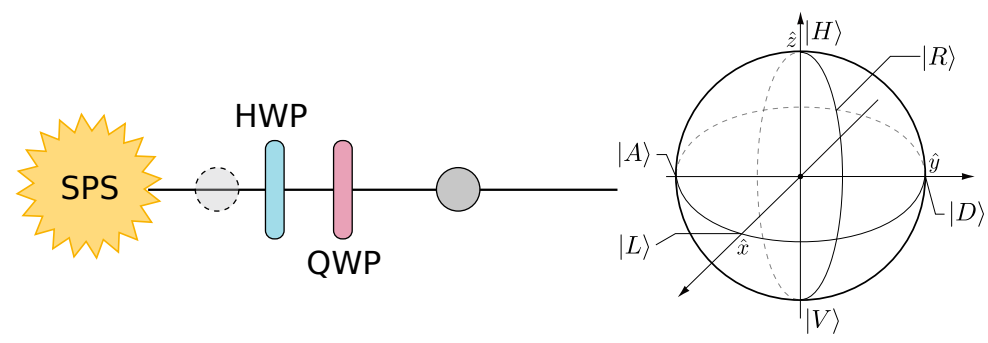

Figure 1.11: Using only common linear optical components found in most photonic laboratories, for instance, a set of of a half-wave (HWP) and a quarter-wave (QWP) plates to transform the polarization state of the emitted single photons. With these apparatuses, any polarization state in the 2-dimensional Hilbert space, represented by a Bloch-Poincaré sphere. 
Feasible manipulation of photonic polarization using elementary linear optical components [bin Abdul Khir 2012] has made it the most popular choice in photonic qubit encoding. By employing, for instance, a combination of a half-wave and a quarter-wave plates, one can engineer any state of polarization in the 2-dimensional Hilbert space (see Fig.1.5). Both qubit states $|0\rangle$ and $|1\rangle$ are represented by the orthogonal polarization of horizontal and vertical, respectively, i.e. $|0\rangle \equiv|H\rangle$ and $|1\rangle \equiv|V\rangle$. Meanwhile, by simply rotating the half-wave plate, we can define the diagonal and anti-diagonal polarization states $|D\rangle \equiv \frac{1}{\sqrt{2}}(|0\rangle+|1\rangle)$ and $|A\rangle \equiv \frac{1}{\sqrt{2}}(|0\rangle-|1\rangle)$, respectively. Eventually, thanks to the rotating quarter-wave plate we can have access to circular-left and circular-right polarization states, $|L\rangle \equiv \frac{1}{\sqrt{2}}(|0\rangle+i|1\rangle)$ and $|R\rangle \equiv \frac{1}{\sqrt{2}}(|0\rangle-i|1\rangle)$, respectively. Polarization-encoded photonic qubits can be conveniently analyzed using a polarizing beam splitter (PBS) and wave-plate followed by single photon counting devices. However, when the aim is to demonstrate quantum communication over long distances, the use of optical fibers as the channel medium is undeniable. Unfortunately, in optical fibers, photons suffer from polarization drifts and dispersion due to fiber birefringence itself and birefringence fluctuation induced by the enviromental changes. However, active compensation of fiber birefringence fluctuations can be now be adopted to ensure the overall polarization stability in optical fibers [Xavier et al. 2009], [Chen et al. 2007], [Chen et al. 2009].

\section{Phase encoding}

In the early days of quantum communication, researchers introduced phase-encoded photonic qubits. Back then, phase-shift keying was already a conventional method of digital modulation used in standard telecommunication, not to mention the readily available, highperformance, electrically-controlled phase modulators. Furthermore, the phase of a single photon is much more stable in optical fibers compared to polarization. These are the reasons why phase encoding is preferred for long distance quantum communication. The most basic implementation of phase coding can be done using a deployed balanced Mach-Zehnder interferometer configuration where single-photon interference effect can be observed (see Fig.1.12). In this case, the qubit states can be associated with the phase difference between the two arms of the interferometer. For example, the state $|0\rangle$ represents the case where no phase difference is observed while the state $|1\rangle$ denotes a phase difference of $\pi$. In the 


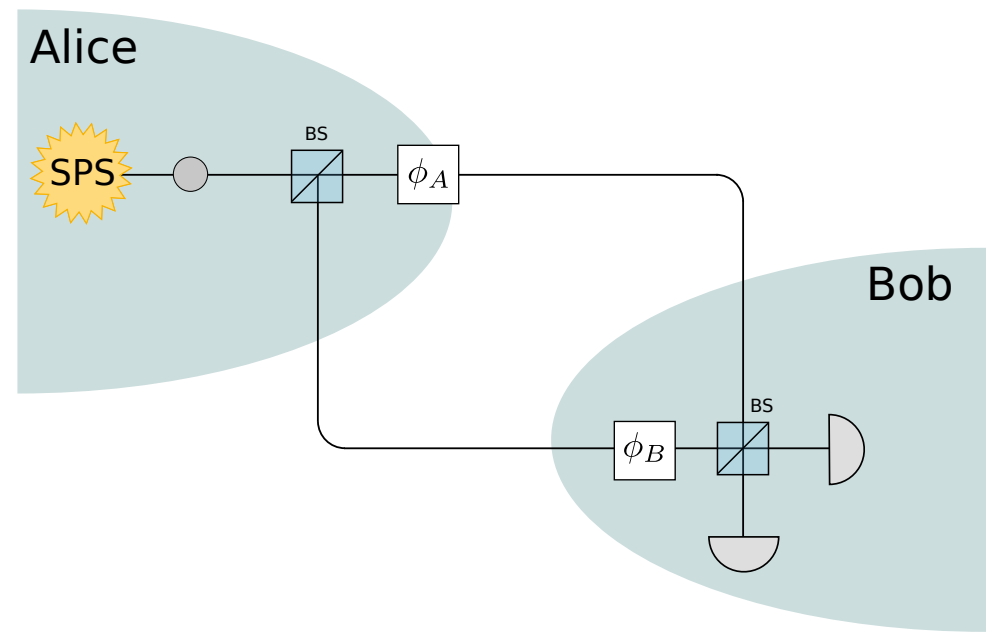

Figure 1.12: The most simplistic model of phase encoding technique used in quantum communication. Photons produced by a single photon source (SPS) are injected one by one into a balanced Mach-Zehnder interferometer. The encoding and decoding of quantum information are performed with the help of two phase modulators, $\phi_{A}$ and $\phi_{B}$. In the case of compatible bases, $\phi_{A}-\phi_{B}$ gives $\pi$ or zero. For the complementary bases, $\phi_{A}-\phi_{B}$ gives $\frac{\pi}{2}$ or $\frac{3 \pi}{2}$.

principle of quantum communication, the control of the phase encoding-decoding by two communicating parties is performed with the help of two phase modulators, located in each arm of the interferometer and at each user's location. With this technique, there are two major conditions that need to be fulfilled:

- The coherence length of the single photons $\mathcal{L}_{\text {coh }}^{\text {photon }}$ should be longer than the path length difference between the two arms $(\Delta L)$ so as to ensure single-photon interferencecan occur. This condition reads $\mathcal{L}_{\text {coh }}^{\text {photon }} \gg \Delta L$.

- $\Delta L$ of the interferometer needs to be kept stable during the measurement for avoiding interference pattern fluctuations.

\section{Time-bin encoding}

However, researchers were much more concerned with the latter (phase drift problem). The resolution to this problem is actually to reduce the individual phase shift experienced by each arm to the minimum [Townsend et al. 1993]. This can be done by simply joining together, for the most part, the two arms in the same spatial mode. For this, two additional 
beam splitters can be used and eventually we end up in a dual-interferometer configuration as depicted in Fig.1.13. In this case, one interferometer is used for encoding and the other one for decoding the quantum information.

Interestingly, the use of dual-interferometer configuration has led to the demonstration of another type of photonic qubits. Time-bin qubits were actually first demonstrated in Geneva [Brendel et al. 1999] and till this very day, such qubits are considered to be the best candidates for battling the decoherence effects in optical fibers. As the name suggests, the encoding of information can be performed considering the arrival times of the qubits. For example, if an optical pulse containing one photon is injected into an unbalanced MachZehnder interferometer, there will be only two possibilities: either the photon takes the long arm or the short arm. Consequently, we are dealing with two output states namely the early and the late time-bins which can further be associated with states $|0\rangle$ and $|1\rangle$, respectively. Obviously in this case, single-photon interference needs to be avoided, both in the preparation and analysis unbalanced Mach-Zehnder interferometers at Alice's and Bob's locations, respectively $\left(\mathcal{L}_{\text {coh }}^{\text {photon }} \ll \Delta L_{A, B}\right)$. A simple measurement of analyzing the time interval between the trigger signal from optical source and the firing of a single photon detector can easily reveal which arm the detected photon had taken previously.

For the moment, the qubits are prepared only in $\{|0\rangle ;|1\rangle\}$ basis. By adding a phase modulator, we can have access to the phase coding capabilities. Moreover, by replacing beam splitter in the interferometer by a variable one we can conveniently produce any pure qubit state within 2-dimensional Hilbert space (see Fig.1.13). To decode such timebin information in different basis, the output photon is directed toward a second, equally similar interferometer. From the very beginning, for the injected photon to arrive at the last beam splitter, there are four different paths that the photon can propagate through. Within these possibilities, there are two paths that interfere, thus no which-path information can be gained. Those paths are $l_{A} s_{B}$ and $s_{A} l_{B}$, where $s$ and $l$ denote short and long paths of the interferometer, and $A$ and $B$ stand for Alice and Bob, respectively. Nonetheless, this serves as another orthogonal measurement basis of $\left\{\frac{1}{\sqrt{2}}\left(|0\rangle+e^{i \Phi}|1\rangle\right), \frac{1}{\sqrt{2}}\left(|0\rangle-e^{i \Phi}|1\rangle\right)\right\}$, where $\Phi$ denotes the phase difference between the two interferometers.

Aside from higher resistance to decoherence in optical fibers, we also benefit from the fact that the time-bin encoding technique is not just limited to qubit [de Riedmatten et al. 2002]. 


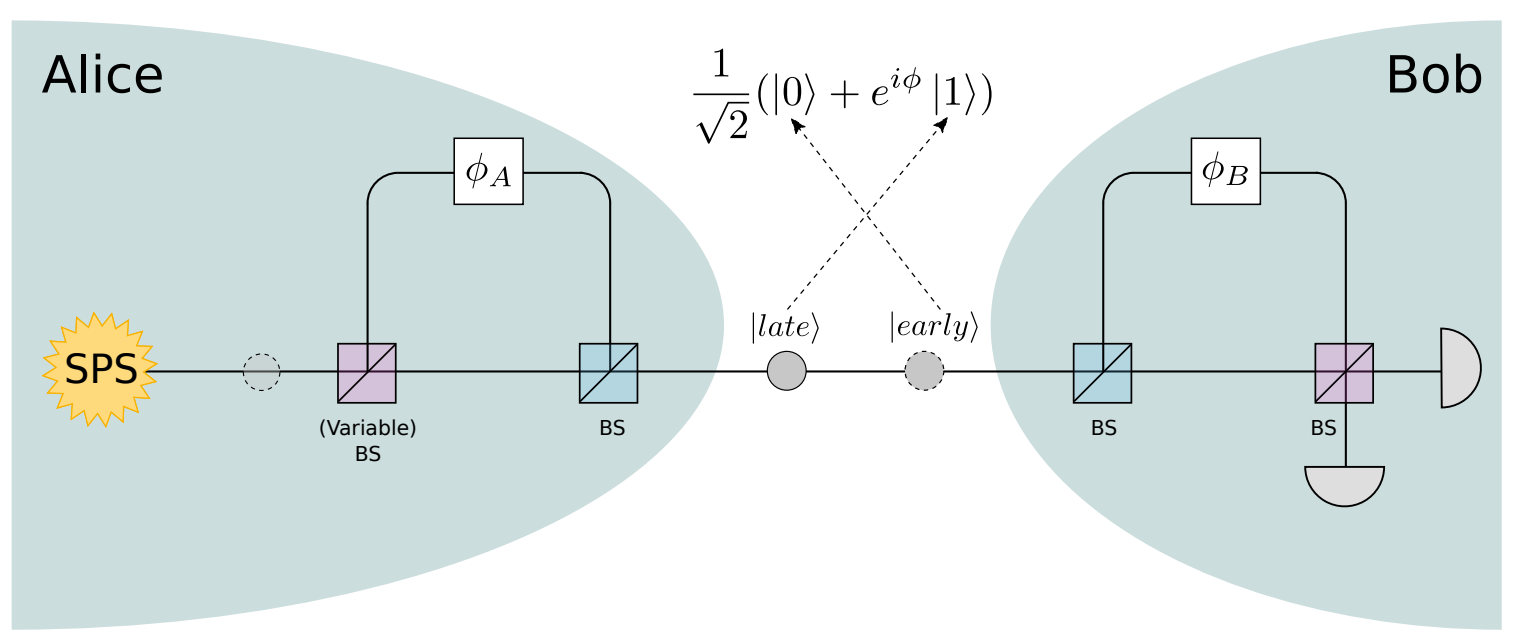

Figure 1.13: Time-bin qubits can be generated considering the arrival times of the carrier single photons. In this example, a single photon is sent to an unbalanced Mach-Zehnder interferometer. At the output of it, the photon will be in the superposition of early and late states $\frac{1}{\sqrt{2}}(\mid$ early $\rangle+e^{i \phi} \mid$ late $\left.\rangle\right) \rightarrow \frac{1}{\sqrt{2}}\left(|0\rangle+e^{i \phi}|1\rangle\right)$. From the previous equation, the phase $\phi$ is defined by the setting of the preparation interferometer, which is the path length difference between the long and the short arms. The encoding-decoding of quantum information are performed by the help of variable beam splitters and phase modulators.

As opposed to the polarization observable, it is much easier to create the high-dimensional time-bins and this can be achieved simply through the use of unbalanced interferometer configuration. Moreover, time-bin observable has also been used in linear optical quantum computing applications [Humphreys et al. 2013].

\section{Other qubit encoding strategies}

Among various photonics observables, one also can use, for instance, frequency-bin or angular momentum qubits for quantum communication and processing. With frequencybin observable, qubit manipulation is done through the implementation of acousto-optical modulators or combinations of electro-optical modulators with optical filters [Olislager et al. 2010b, Olislager et al. 2010a, Olislager et al. 2011, Ramelow et al. 2013]. Meanwhile, angular momentum qubits may not represent a household name, particularly in quantum communication. However, more and more interests have been given recently toward angular momentum at single photon level [Mair et al. 2001, Lea 2002, Nagali et al. 2009, Nicolas et al. 2014]. 


\subsubsection{Secret key establishment, "quantumly"}

So far, we have witnessed the concept of quantum randomness in measurement outcomes due to quantum superposition which can be further elaborated with the use of complementary bases. On top of that, we know already that an unknown qubit state cannot be perfectly cloned. How come researchers invest so much interest in these weird aspects of quantum mechanics? The answer is simple: beyond the no-go theorems of quantum physics, physicists discovered a means to exploit them for advanced quantum applications, quantum key distribution (QKD) being part of these.

Earlier in this chapter, we have discussed the limitations of classical information in terms of data exchange security. For instance, an ideal implementation of the Vernam's one-time pad (OTP) method requires randomness in the key creation and the key itself must be kept secret only between Alice and Bob. Interestingly, the resolution to these two problems actually resides in the quantum world. We will shortly introduce the underlying ideas of QKD. As QKD is ruled by the quantum aspects discussed above, a key can be constructed with true randomness and simultaneously, it can be distributed secretly between Alice and Bob with the certainty of detecting any attempt of eavesdropping. With the generated key, messages can be conveniently encrypted and decrypted using the powerful OTP method.

\subsubsection{The ideal case of BB84}

The BB84 protocol, invented by Charles Bennett and Gilles Brassard in 1984 [Bennett \& Brassard 1984] (see Fig.1.14), is the most famous among the various QKD protocols as it was the first to be discovered and as it permitted to actually launch the field. The essence behind BB84 is quite easy to understand from what has been presented so far in this chapter. In this section, the protocol itself will not be discussed thoroughly, but the important mechanism allowing absolutely secure key sharing between Alice and Bob will be highlighted.

The most difficult part of the protocol involves the transmission of a set of truly random qubits from one party to the other. To encode the information, for instance, on the polarization state of a single photon, two complementary bases in the same 2-dimensional Hilbert space are used, such as: 
- Rectilinear basis of $\{H, V\}$.

- Diagonal basis of $\{D, A\}$.

To begin with, Alice randomly chooses between these two bases. In this fashion, every single photon that is transmitted by Alice is truly random in polarization. Although difficult, this can be achieved by using a quantum random number generator (QRNG) to drive the basis selection.

The randomness in qubit preparation is crucial to ensure the security of the protocol. Imagine an eavesdropper Eve is secretly listening to the transmission. For her to gain some information, she is forced to make measurements. However, if the basis selection by Alice is completely random, this will prevent Eve from knowing which basis she must choose for her measurements. For the moment, the best she can do to hide her presence is to regenerate the measured photons and send them back to Bob (commonly known as the intercept-resend attack). Sure, half of the time, her guesses are spot on. But in the other half of the cases, she will definitely introduce errors to the system due to wrong basis selections and this is how her presence can be detected. In the literature, this error rate is defined as quantum bit error rate $(Q B E R)$. For a simple intercept-and-resend attack in BB84 implementation with single photons, $Q B E R$ is equal to $25 \%$.

On the other hand, every transmitted photon is measured by Bob, also in a randomly chosen basis such that even himself does not know whether his measurements yield deterministic or probabilistic results (see Sec.1.2.1.1). Once the whole transmission is over, Bob publicly announces the choice of his bases. Alice will simply reply whether or not he used the correct basis for each measurements. But, neither Alice nor Bob reveals their measurement results. To this point, they reconstitute a string of bits by discarding the cases where Bob's basis is incompatible with that of Alice. Provided there is no interception by an eavesdropper, Alice and Bob should now share a common, genuinely randomized, sifted key.

\subsubsection{BB84 in a noisy channel}

Is the obtained sifted key enough to be used for encrypting a secret message with the OTP protocol? Well, it is not that simple. In reality, the communication channel can 
be considered noisy, where for instance, a transmitted qubit can be flipped with a certain probability. Obviously, this also can contribute to errors. More importantly, we also need to take into account the fact that the mighty Eve may have the possibility to replace the noisy channel with a perfect one such that errors are solely contributed by her action of eavesdropping. In this case, trying to discriminate natural errors from Eve-induced ones is just impossible. Due to the latter, not all uncorrelated bits will be discarded during the key sifting. Still, a smarter way of thinking would be to reconsider all errors are actually originated from Eve.

In the face of such situation, any possible amount of information that can possibly be gained by Eve should be reduced to the minimum. This includes further treatment to the obtained sifted key involving privacy amplification at the price of heavily reduced key rates. However, before performing privacy amplification, all errors in the sifted key need to be removed through an error correction procedure. It is not the scope of this thesis to actually discuss in details the operation associated with error correction procedure and privacy amplification. For further insight into that matter, we would like to invite reader to have a look on a review article referenced in [Gisin et al. 2002].

\subsubsection{Enhancing QKD through Ekert protocol}

A QKD system can also be based on the use of photonic entanglement, as was proposed by Artur Ekert in 1991 [Ekert 1991]. In this case, the photons are no longer prepared by Alice nor Bob. Instead, both receive each a photon from a common, entangled photon pair source (EPPS) located in the middle of the communication channel. Considering, for instance, the polarization observable for a maximally entangled state $\left|\Phi^{+}\right\rangle_{a b}=\frac{1}{\sqrt{2}}\left(|H\rangle_{a}|H\rangle_{b}+|V\rangle_{a}|V\rangle_{b}\right)$, Alice and Bob perform a measurement in one of the two bases (either rectilinear or diagonal basis) where the basis selection is also made completely random (ideal case). Being really similar to the procedure of BB84, a sifted key can be constructed from the correlations in the measurement results featuring only compatible bases. Thanks to the use of entanglement, the mechanism to detect the presence of Eve is based on testing Bell's inequality, as discussed in Sec.1.2.3.3.

Truly, such protocol further benefits highly from entanglement. For instance, one can elegantly eliminate the need of external QRNGs, notably for driving the random measure- 
ment basis selection. By employing a beam splitter and two polarizing beam splitters at both sides of the experiment, we can implement the so-called "passive basis selection" [Rarity et al. 1994], allowing the photons themselves to personally choose between the two complementary measurement bases. Furthermore, even if Eve has a total control over the communication channel whereby she could alternate between the use of an EPPS and a source producing a mixture of states (see Sec.1.2.3) such that extra information can be gained, this can be revealed by verifying, as mentioned above, the so-called $S$ parameter through the Bell's inequality test (see again Sec.1.2.3.3). In the same regard, other eavesdropping activities can also be revealed through the same strategy.

\subsubsection{Recent interests in QKD}

Even though the security of QKD is backed up by quantum theory itself, the compelling idea of unconditional security has somehow been tarnished by the so-called "side-channel" attacks targeting specific components in a QKD system. Worse, due to such attacks, even the "supposed-to-be-absolutely-secure" commercialized QKD system has been hacked [Lydersen et al. 2010a].

To battle all the side-channels, entanglement-based device-independent QKD (DI-QKD) has been introduced [Acín et al. 2007, Gisin et al. 2010, Pütz et al. 2015, Aktas et al. 2015]. In standard QKD, Alice and Bob somehow should have, if not all, some insights regarding their devices. For instance, in Ekert protocol, both Alice and Bob needs to assume that the measurement basis selection is actually reflecting the true intended basis. If their measurement system are fabricated by a third-party provider, they have no choice other than to blindly trust the system. This is only one example among various assumptions that define the level of security concerning any QKD implementations. The aim of DIQKD is to bring assumptions like this to the minimum which in return will strengthen the security. In the meantime, the security of DI-QKD is granted with the violation of Bell's inequality. However, the protocol demands an overall detection efficiency nearing unity $(\geq 0.83)$ so as to close the detection loophole as required by Bell's theorem [Massar et al. 2002, Massar 2002]. This remarkably represents the biggest challenge toward efficient implementation of DI-QKD protocol.

However, it is realized that most of demonstrated side-channel attacks on a QKD system 
are detector-related. These mainly involves the time-shift [Qi et al. 2007, Zhao et al. 2008], the bright illumination [Makarov 2009, Lydersen et al. 2010a, Lydersen et al. 2010b, Lydersen et al. 2011, Wiechers et al. 2011], the device calibration [Jain et al. 2011] and the detector dead-time [Weier et al. 2011] attacks. Based on this fact, researchers have came up with an alternative solution which is, in a sense, more realistic. The measurement-deviceindependent QKD (MDI-QKD) [Lo et al. 2012, Valivarthi et al. 2015] basically involves the Bell state measurement by an untrusted third-party, thus eliminating all the side-channels related to the detector, provided the sources are trusted. Apart from that, MDI-QKD has another underlying advantage. It paves the way toward efficient quantum networking with untrusted nodes due to the fact that its building blocks are identical to that of quantum repeaters. Consequently, we are indeed one step closer to the realization of satellite-toground QKD as the untrusted satellite issue can now be resolved with the implementation of MDI-QKD.

For the moment, both DI-QKD and MDI-QKD represent hot topics in the field of QKD. 


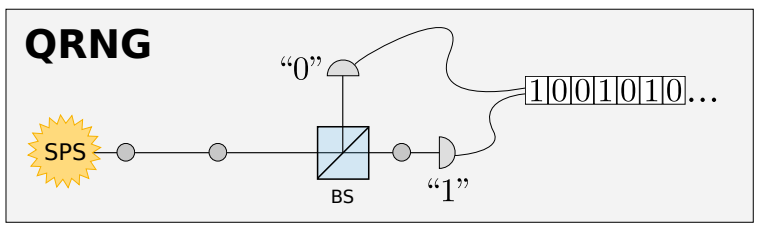

\section{QRNG}

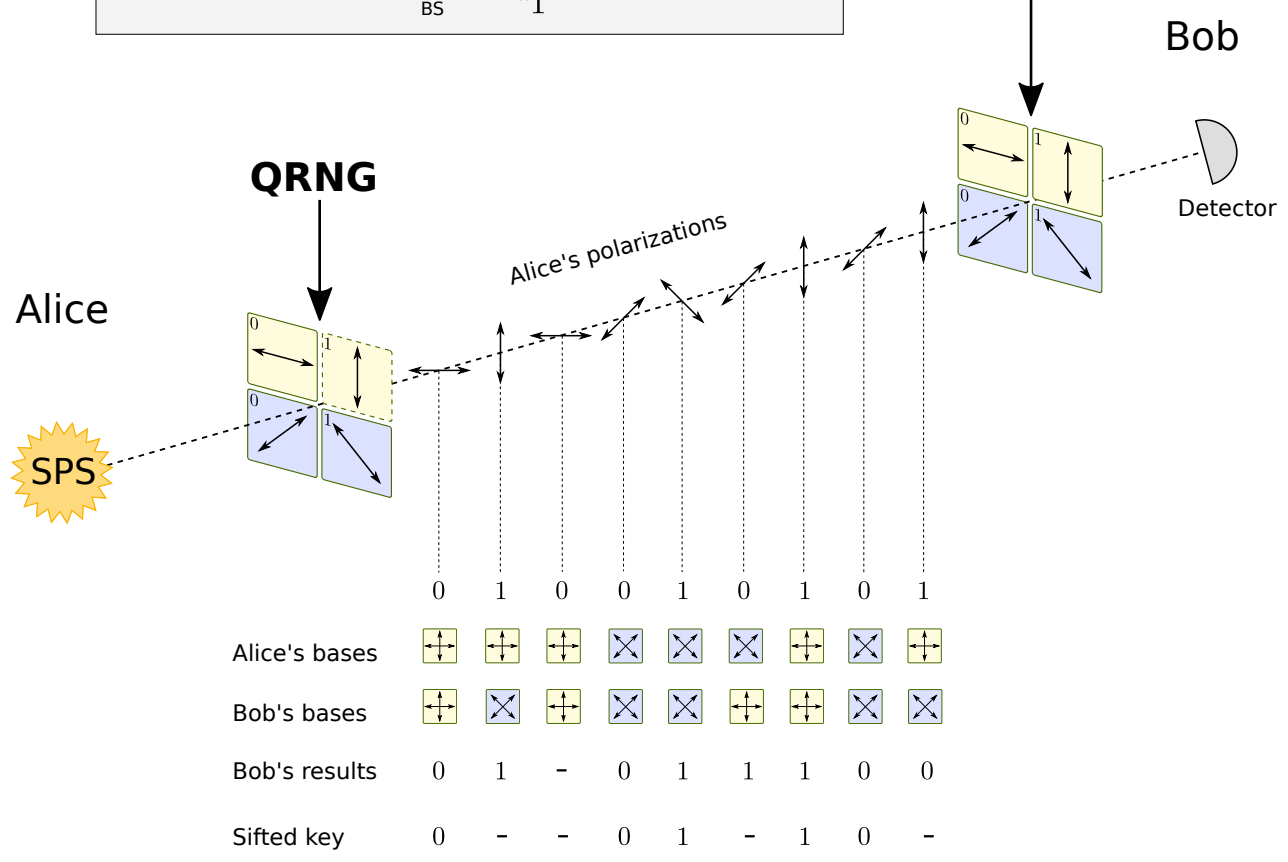

Figure 1.14: The working principle of BB84 QKD protocol. Alice encodes her qubits randomly using one of the complementary bases, i.e. using rectilinear or diagonal orientations. On the other hand, Bob measures the incoming qubits also in a random manner. Only the bits corresponding to compatible bases between Alice and Bob are kept $\rightarrow$ sifted key. In this example, the random selection of preparation and measurement bases are driven by a separate quantum random number generator (QRNG). In the above gray box labeled QRNG, we find an illustration of random bit generation based on the superposition of two paths. Once the emitted single photon is incident on a beam splitter (BS), the photon will be detected in either detector with an equal probability of $\frac{1}{2}$. If we discriminate the detection events between the upper and lower detectors with a binary representation, a pure random string of bits can be produced. As a side note, QRNG can also be based on more efficient methods namely the vacuum noise [Gabriel et al. 2010] and the laser phase noise [Qi et al. 2010a] [Xu et al. 2012] [Nie et al. 2015]. 


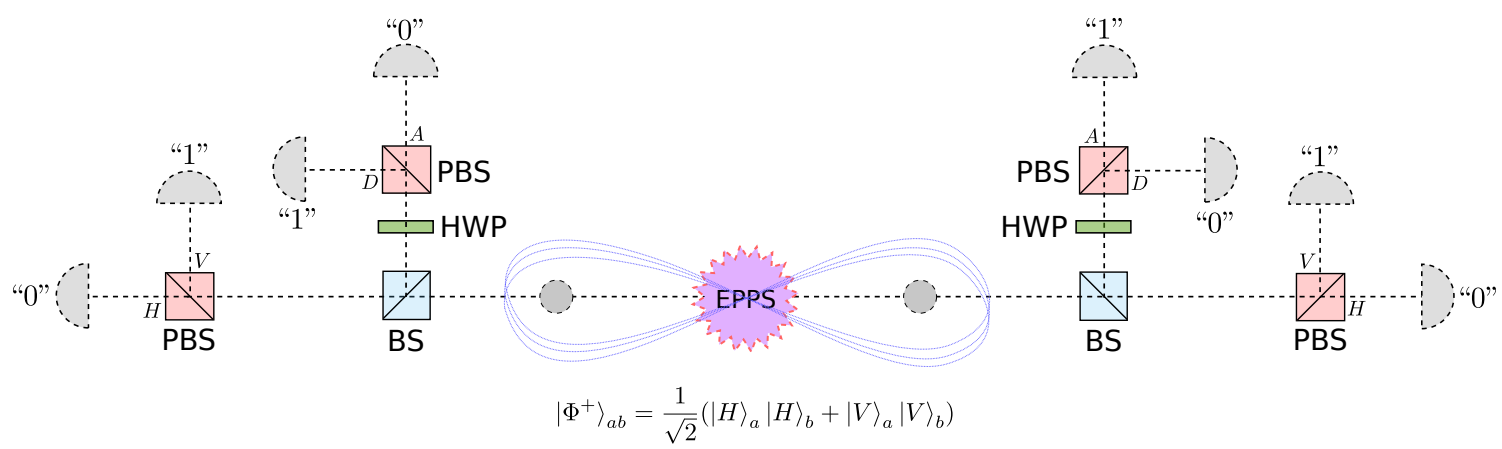

Figure 1.15: Example of Ekert QKD protocol highlighting "passive basis selection". An entangled photon pair source (EPPS) is employed in the middle of the communication channel. The source emits entangled photons in the state of $\left|\Phi^{+}\right\rangle_{a b}=\frac{1}{\sqrt{2}}\left(|H\rangle_{a}|H\rangle_{b}+\right.$ $\left.|V\rangle_{a}|V\rangle_{b}\right)$. Both Alice and Bob will receive and measure each a photon from the same pair. In this example, the random selection of measurement bases are performed through the so-called "passive basis selection", i.e. the need of external quantum random number generators is eliminated. In the case of Ekert protocol, the key is constructed from correlated measurement outcomes between Alice and Bob featuring only compatible bases. 
Chapter 2

\section{Towards long-distance quantum}

\section{communication}

\section{Contents}

2.1 Pushing the "quantum" distance ............... 47

2.1.1 The case of one section: one-way BB84 . . . . . . . . . . . . 49

2.1.2 The case of two sections: entanglement-enabled Ekert protocol . . . . 51

2.1.3 The case of three or more sections: the quantum relay . . . . . . . . . 52

\subsection{Pushing the "quantum" distance}

Regarding QKD, the short distance of which a secret key can be established represents its major limitation. Evidently, dark counts in single photon detectors and losses in optical fibers contribute much to such constraint. Even though photons at telecom wavelength face less absorption in conventional optical fibers (typically around $0.2 \mathrm{~dB} / \mathrm{km}$ at $1550 \mathrm{~nm}$ ), establishing a QKD link beyond hundreds of kilometers is simply something that is difficult to realize. One should also not forget, simply amplifying the light is of no help due to the no-cloning theorem (see Sec.1.2.2).

However, beyond such losses, there lie other factors at play. The fragile aspect of a QKD system can also be related to:

- Imperfect single photon sources. One of the possible attack exploiting the imperfection of a single photon source is the photon number splitting (PNS) attack [Lütkenhaus 2000]. This type of attack could happen if the single photon source emits more 
than just single photon at a given time. This will give Eve the possibility to "steal" one (or a portion) of the transmitted photons. Consequently, Eve can perform her measurement on the stolen photon without even affecting the measurement at Bob's. Closing the window of such vulnerability would require the use of better single photon sources. In recognizing how important this is to the advancement of $\mathrm{QKD}$, it is one of the commitments of this thesis to answer such demand. A state-of-the-art review, along with not one, but two realizations of high-performance single photon sources will be demonstrated thoroughly in Chapters 6, 7 and 8 .

- Imperfect single photon detectors. This represent another critical point that we should pay close attention to. Aside of having the reputation of being the "weakest link" in the security of a QKD system (see Sec.1.2.5.4), most of the single photon detectors, notably in the telecom range of wavelength, exhibit low quantum detection efficiency and high dark count rates. For a detector, having the efficiency below unity simply translates to the extra losses gained by the system which further reduces the maximum achievable key rates. Meanwhile, the dark counts are classified as the detection events in the absence of actual photons which can lead to errors in the communication and limited, or reduced, signal-to-noise ratios (SNR). Notably, after a certain propagation distance, the probability of detecting the photons will plunge below the intrinsic dark count level of the employed detector, leading to irreversible loss of information.

It is an ugly truth that QKD is too dependent on technology. Slow technological improvement considering huge financial and time constraints has indeed stagnated the advancement of QKD in term of maximum achievable distances. This is where quantum entanglement comes in handy. One important point that has been left out from the discussion of the Ekert protocol (see Sec.1.2.5.3) is the fact that the produced correlated photons by the entangled photon pair source are highly synchronized. This opens the possibility for Alice and Bob to perform their measurements in terms of coincidences. Here, the advantage would be the increase in the signal-to-noise ratio (SNR) due to the decrease of the probability of registering, simultaneously, a dark count in each associated detectors, such that higher separation distance between Alice and Bob is attained. This brings us to the introduction of the quantum relay concept. 
Next, we are going to calculate, for each scenarios, from the one-way implementation of BB84 protocol down to quantum relay configuration consists of multiple sections, the secret key rate $r$ as a function of the distance $d$ separating two communicating parties, Alice and Bob.

a)

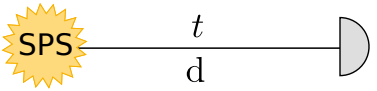

b)

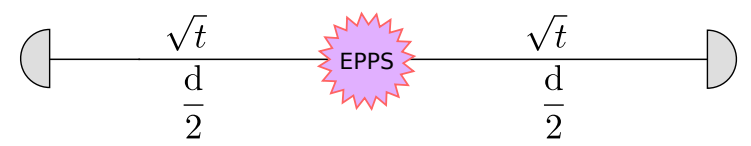

c)

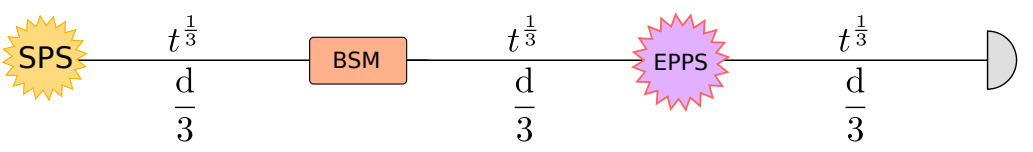

d)

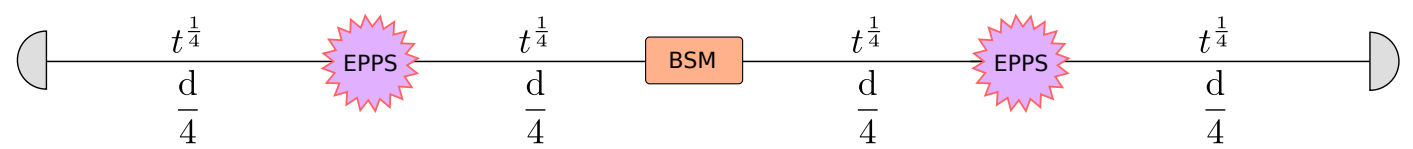

Figure 2.1: Illustrations of different scenarios for quantum communication. (a): The most simplest form of point-to-point communication involving two communicating parties. Single photons emitted by the single photon source (SPS) are directly sent to the destination. (b): Both communicating parties share a quantum resource which is located in the middle of the communication channel. Here, an entangled photon pair source (EPPS) emits entangled photons in two different directions toward Alice and Bob. (c) and (d): The cases of quantum relay. The enabling quantum protocols for (c) and (d) are quantum teleportation and the entanglement swapping, respectively. In the those graphics, BSM stands for Bell state measurement, or joint measurement. BSM is capable of projecting two independent single photons, when sent to a 50/50 beam splitter, onto an entangled state.

\subsubsection{The case of one section: one-way BB84}

We will first consider, for instance, the implementation of the BB84 QKD protocol discussed in Sec.1.2.5.1 in this chapter. As has been depicted in Fig.2.1 (a), the one-way communication involves the unidirectional transmission of Alice's qubits generated by a single photon source to Bob. Before proceeding further, it is necessary to highlight some important remarks:

- We define the transmission of the communication channel as $t$ parameter and $t$ is actually none other than the probability of successfully transmitting a photon. We 
also define $\alpha$ as the losses in the optical channel for which $\alpha=0.2 \mathrm{~dB} / \mathrm{km}$ (typical value involving the use of optical fibers at telecom wavelength). In this case, the transmission $t$ can further be described as $t=10^{\frac{-\alpha \cdot d}{10}}$ where $d$ represents the distance separating Alice and Bob in km.

- The use of avalanche photo-diodes (APDs) based on indium-gallium-arsenide (InGaAs) represents the most common approach toward detecting single photons at telecom wavelength (1300-1600 nm). Famous for its poor performances, this kind of APD is usually limited to a typical value of $20 \%$ in term of quantum detection efficiency $(\eta=0.2)$. Such APDs also exhibit a dark count probability $P_{D C}$ of $10^{-6} / \mathrm{ns}$.

From the discussed parameters, we begin with defining the probability of detecting a photon at the end of the line which is

$$
P_{\text {signal }}=t \cdot \eta
$$

Meanwhile, the probability of registering a false detection $P_{\text {noise }}$ depends on the followings:

- The transmitted photon is left undetected $\rightarrow t(1-\eta) P_{D C}$.

- No transmission of photon at all $\rightarrow(1-t) P_{D C}$.

Therefore, $P_{\text {noise }}$ reads

$$
P_{\text {noise }}=t(1-\eta) P_{D C}+(1-t) P_{D C}=(1-t \cdot \eta) P_{D C}
$$

Consequently, the probability of having any detection by the APD is given by

$$
P_{\text {det }}=P_{\text {signal }}+P_{\text {noise }}
$$

For a secure communication, a certain level of errors in the transmission can be tolerated depending on the protocol itself. If this level is too high, it may suggest that the errors are not all natural, but rather, some might be caused by Eve. This threshold can be expressed 
in term of the maximum tolerable quantum bit error rate $(Q B E R)$

$$
Q B E R=\frac{P_{\text {noise }}}{P_{\text {det }}+P_{\text {noise }}} .
$$

Throughout this section, we will consider a general threshold for QBER of $15 \%$ under individual attacks assumption [Gisin et al. 2002]. Eventually, the QBER leads us to the calculation of the key rates $r$,

$$
r=P_{\text {det }}\left(1-\frac{Q B E R}{Q B E R_{\text {thres }}}\right)=P_{\text {det }}-\frac{P_{\text {noise }}}{Q B E R_{\text {thres }}} .
$$

In a one-way implementation of BB84 protocol (see Fig.2.2), the key rate at zero distance implies the loss-less scenario of the transmission channel thus the produced rate depends heavily on the APD's efficiency. The key rate then drops linearly as a function of the losses $\alpha$ until a point where there is a sudden collapse due to signal-to-noise ratio in the transmission equals or below unity, i.e. the probability to detect a single photon is comparable, or below, the intrinsic dark count level of the employed APD. Before the collapse, the distance can go up to $160 \mathrm{~km}$ (see Fig.2.2).

\subsubsection{The case of two sections: entanglement-enabled Ekert protocol}

Here, we consider the Ekert protocol depicted in Sec.1.2.5.3 where a photon pair source is employed to provide Alice and Bob entangled qubit. In this case, the secret key is constructed from the correlations between Alice's and Bob's respective measurements. Therefore, a valid bit can only be obtained through the detection from both detectors (issued by photons from the same pair). Accordingly, the probability of detecting the transmitted photons at both extremities $P_{\text {signal }}^{2 \text { sec }}$ is given by

$$
P_{\text {signal }}^{2 \text { sec }}=t \cdot \eta^{2}
$$

Meanwhile, by taking into account the following cases:

- One photon is successfully detected by Alice at the same time as the detection of a dark count in Bob's detector, or the other way around $\rightarrow 2 \sqrt{t} \eta(1-\sqrt{t} \eta) P_{D C}$. 
- The coincidence is due only to dark counts in both detectors, for which both photons might get lost along the communication channel or simply not detected $\rightarrow(1-\sqrt{t} \eta)^{2} P_{D C}^{2}$.

We can define the probability of registering a false coincidence $P_{\text {noise }}^{2 s e c}$, such that

$$
\begin{aligned}
P_{\text {noise }}^{2 \text { sec }} & =2 \sqrt{t} \eta(1-\sqrt{t} \eta) P_{D C}+(1-\sqrt{t} \eta)^{2} P_{D C}^{2} \\
& =\left[\sqrt{t} \eta+(1-\sqrt{t} \eta) P_{D C}\right]^{2}-t \eta^{2} .
\end{aligned}
$$

Eventually, by injecting $P_{\text {signal }}^{2 s e c}$ and $P_{\text {noise }}^{2 s e c}$ into Eq.(2.5), we can see that the maximum achievable distance for Ekert protocol is considerably increased (up to $320 \mathrm{~km}$ ) compared to the one-way implementation of BB84 protocol (see Fig.2.2). However, due to lower probability of detecting a true coincidence, the key rate is lower than the previous case.

\subsubsection{The case of three or more sections: the quantum relay}

By shifting toward the use of an EPPS, the number of section through which (entangled) single photons propagate is increased which leads to overall longer achievable communication distances. The underlying factor that directly contributes to such advantage is the use of coincidence type detection, such that the probability of registering, simultaneously, a dark count in each associated detectors becomes very low. In other words, this strategy provides an increase of SNR or at similar SNR, hence higher maximum achievable distances. Thus, it is relatively intuitive to presume that the more sections are involved within a communication link, i.e. emitting and detecting the $n$ single photons, the farther the separation is between Alice and Bob.

For a communication link involving $n$-section, the probability of having a good detection reads

$$
P_{\text {signal }}^{n-s e c}=t \cdot \eta^{n}
$$

while the probability of having a false detection is given by

$$
P_{\text {noise }}^{n-s e c}=\left[t^{\frac{1}{n}} \eta+\left(1-t^{\frac{1}{n}} \eta\right) P_{D C}\right]^{n}-t \eta^{n}
$$


To further increase the distance (see Fig.2.2), we can resort, for instance, to the implementation of quantum relay configuration within the framework of three or more sections. Therefore, it is imperative to define the enabling quantum protocols making those situations possible. For that purpose, we will next discuss the quantum teleportation (see Sec.2.1.3.1) and the teleportation of entanglement (see Sec.2.1.3).

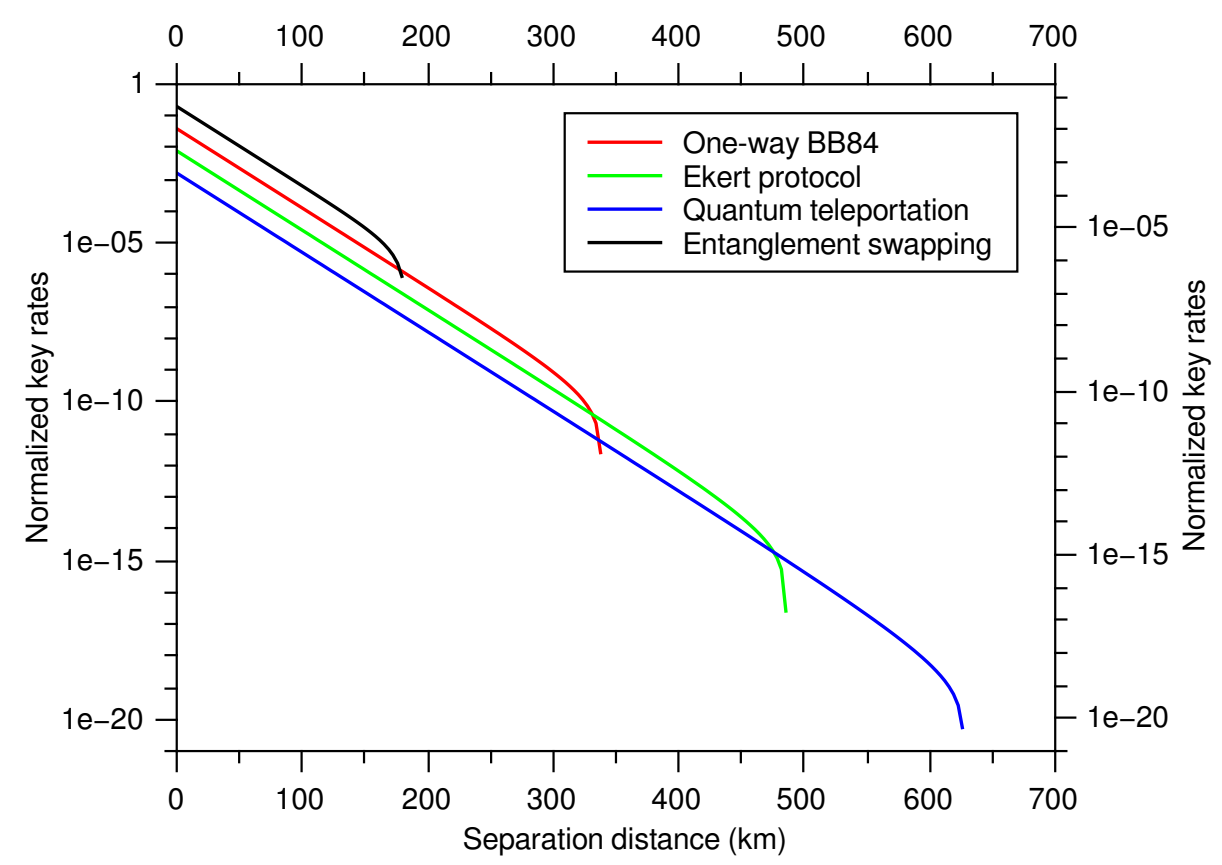

Figure 2.2: The generated key rates as a function of the separation distance between two communicating parties for different scenarios. The following parameters are adopted for the simulation purposes: the losses of optical channel $\alpha=0.20 \mathrm{~dB} / \mathrm{km}$, the quantum efficiency of employed detectors $\eta=20 \%$, the detectors' dark count probability $P_{D C}=10^{-6} / \mathrm{ns}$ and a QBER threshold of $15 \%$.

\subsubsection{Quantum teleportation}

Two best friends at remote locations want to establish a quantum link. For that purpose, the receiver Bob asks the sender Alice to prepare an unknown qubit in the general state (pure state) of

$$
|\psi\rangle_{1}=\alpha|0\rangle_{1}+\beta|1\rangle_{1}
$$

where the normalization of $|\alpha|^{2}+|\beta|^{2}=1$ is satisfied. Unfortunately, Alice cannot send her prepared qubit $|\psi\rangle_{1}$ directly to Bob because they are not directly connected. However, 
they are somehow joined, indirectly, by an entangled photon pair source (EPPS) located in the middle such that Alice and Bob share an entangled state that reads

$$
|\psi\rangle_{23}=\frac{1}{\sqrt{2}}\left(|0\rangle_{2}|0\rangle_{3}+|1\rangle_{2}|1\rangle_{3}\right)
$$

where the subscripts 2 and 3 represent the individual photons. Consequently, both Alice and Bob are found in a situation as depicted in Fig.2.3.

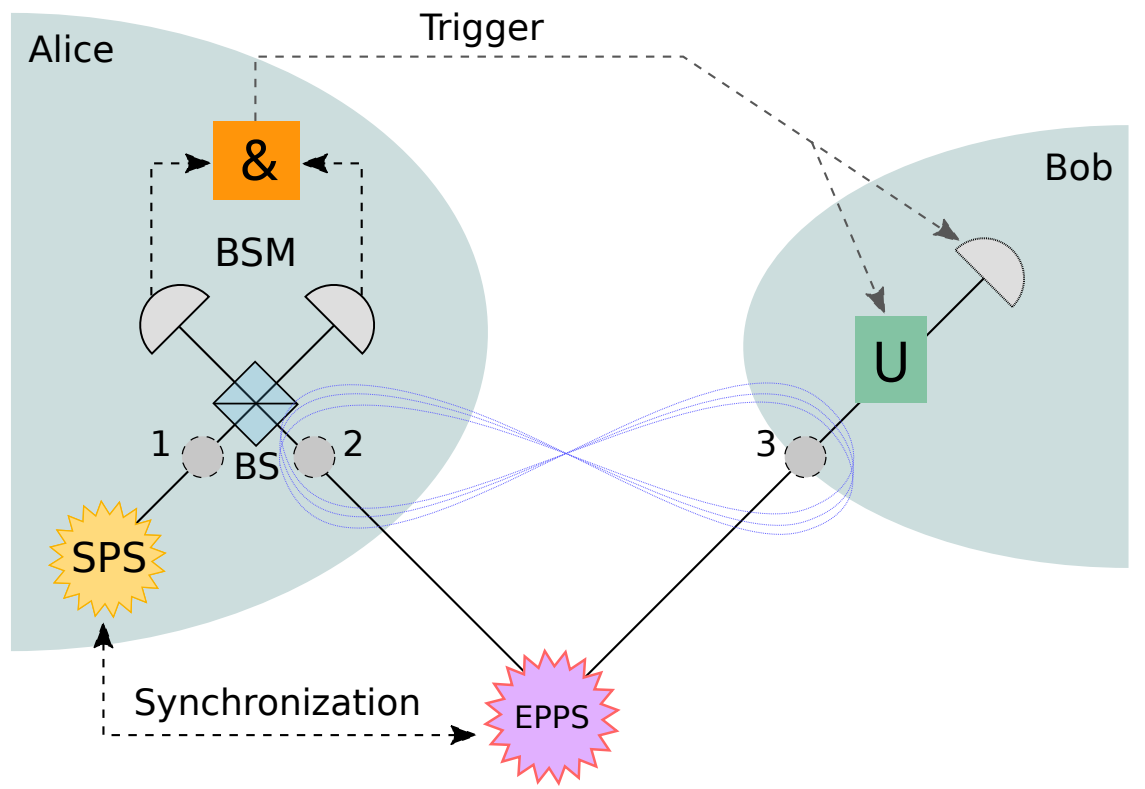

Figure 2.3: Schematic diagram showing the principle of quantum teleportation. Teleportation involves the use of two quantum resources, a single photon source (SPS) and an entangled photon pair source (EPPS). With the SPS, Alice prepares the photon 1 which carries an unknown qubit $|\psi\rangle_{1}=\alpha|0\rangle_{1}+\beta|1\rangle_{1}$. In the meantime, the EPPS emits a photon pair consists of photons 2 and 3 defined in the entangled state $|\psi\rangle_{23}=\frac{1}{\sqrt{2}}\left(|0\rangle_{2}|0\rangle_{3}+|1\rangle_{2}|1\rangle_{3}\right)$. Together with photon 1 and 2, Alice performs a Bell state measurement (BSM) which allows projecting both photons on one of the four maximally entangled Bell states $\left(\left|\Phi^{+}\right\rangle_{12}\right.$, $\left|\Phi^{-}\right\rangle_{12},\left|\Psi^{+}\right\rangle_{12},\left|\Psi^{-}\right\rangle_{12}$, see Eq.(1.16)). The outcome of such measurement will produce an electrical signal, i.e. a trigger, alerting Bob on when to open his detector and on the choice of the unitary transformation $U$ that he has to apply on photon 3. BS: $50 / 50$ beam splitter.

Thanks to the shared quantum resource, Alice can now "transfer" the state of her qubit to Bob without letting go the originally prepared qubit. This trick is famously known as the quantum teleportation protocol [Bennett et al. 1993]. In other words, teleportation involves the transfer, through entanglement, of an independent single qubit onto another photon initially entangled with a third one. 
As Alice and Bob both deal with three-qubit, i.e. tripartite system, issued by two independent quantum systems, they can define a product state $|\psi\rangle_{123}$ that reads

$$
\begin{aligned}
|\psi\rangle_{123} & =|\psi\rangle_{1} \otimes|\psi\rangle_{23} \\
& =\frac{1}{\sqrt{2}}\left(\alpha|0\rangle_{1}|0\rangle_{2}|0\rangle_{3}+\alpha|0\rangle_{1}|1\rangle_{2}|1\rangle_{3}+\beta|1\rangle_{1}|0\rangle_{2}|0\rangle_{3}+\beta|1\rangle_{1}|1\rangle_{2}|1\rangle_{3}\right)(2.2
\end{aligned}
$$

By rewriting the computational basis as:

$$
\left\{\begin{aligned}
|0\rangle|0\rangle & =\frac{1}{\sqrt{2}}\left(\left|\Phi^{+}\right\rangle+\left|\Phi^{-}\right\rangle\right), \\
|0\rangle|1\rangle & =\frac{1}{\sqrt{2}}\left(\left|\Psi^{+}\right\rangle+\left|\Psi^{-}\right\rangle\right), \\
|1\rangle|0\rangle & =\frac{1}{\sqrt{2}}\left(\left|\Psi^{+}\right\rangle-\left|\Psi^{-}\right\rangle\right), \\
|1\rangle|1\rangle & =\frac{1}{\sqrt{2}}\left(\left|\Phi^{+}\right\rangle-\left|\Phi^{-}\right\rangle\right)
\end{aligned}\right.
$$

Now, we can conveniently introduce the four Bell states in the tripartite state $|\psi\rangle_{123}$ such that

$$
\begin{aligned}
|\psi\rangle_{123}= & \frac{1}{2}\left(\left|\Phi^{+}\right\rangle_{12}\left(\alpha|0\rangle_{3}+\beta|1\rangle_{3}\right)+\left|\Phi^{-}\right\rangle_{12}\left(\alpha|0\rangle_{3}-\beta|1\rangle_{3}\right)\right. \\
& \left.+\left|\Psi^{+}\right\rangle_{12}\left(\beta|0\rangle_{3}+\alpha|1\rangle_{3}\right)+\left|\Psi^{-}\right\rangle_{12}\left(-\beta|0\rangle_{3}+\alpha|1\rangle_{3}\right)\right) .
\end{aligned}
$$

The principle of quantum teleportation protocol can be understood through the interpretation conveyed by Eq.(2.14). From this equation, it is found already that for each projected Bell states for photons 1 and 2, the state of photon 3 appears really close to that initial state of photon 1 .

The Bell state measurement (BSM) done by Alice permits projecting, randomly, photons 1 and 2 onto one of the four maximally entangled Bell states with an equal outcome probability of $\frac{1}{4}$. As of the moment, if measured, the state of photon 3 will also appear random to Bob unless this particular photon is acted upon a specific unitary transformation $U$. For this reason, Alice must somehow inform her measurement results to Bob through a classical channel. Based on the information provided by Alice, Bob can conveniently choose an appropriate unitary transformation (see Tab.2.1) so as to recover, by "teleportation", the initial state carried by photon 1 .

The quality of teleportation experiments is gauged upon the fidelity which corresponds to 
Table 2.1: The relations between the results obtained through the Bell state measurement (BSM) of photon 1 and 2, and the choice of suitable $U$ transformation on photon 3. $\sigma_{x, y, z}$ are the two-qubit Pauli operators.

\begin{tabular}{lll}
\hline $\begin{array}{l}\text { Projected Bell } \\
\text { states for } \\
\text { photon } 1 \text { and } 2\end{array}$ & Suitable $U$ transformation & $\begin{array}{l}\text { Corresponding } \\
\text { operators }\end{array}$ \\
\hline$\left|\Phi^{+}\right\rangle_{12}$ & nothing & $I$ \\
$\left|\Phi^{-}\right\rangle_{12}$ & changing of the superposition's sign & $\sigma_{z}$ \\
$\left|\Psi^{+}\right\rangle_{12}$ & swapping of the superposition coeff. ${ }^{1}$ between $|0\rangle$ and $|1\rangle$ & $\sigma_{y}$ \\
$\left|\Psi^{-}\right\rangle_{12}$ & both transformations mentioned above & $\sigma_{x}$ \\
\hline
\end{tabular}

the overlap of the teleported state and the initial state to be teleported. For an ideal case, the protocol should yield a unit fidelity, thus at the same time allows increasing the separation distance between Alice and Bob (see Fig.2.2). So far, experimental demonstrations of the quantum teleportation protocol have been reported. Among others, we find in the literature [Boschi et al. 1998] which dealt with polarization qubit and is recognized to be the very first implementation of teleportation, other polarization qubit-based demonstrations include [Bouwmeester et al. 1997, Ma et al. 2012a, Yin et al. 2012, Bussières et al. 2014], and several time-bin implementations such as [Marcikic et al. 2003, de Riedmatten et al. 2004a, van Houwelingen et al. 2006, Takesue et al. 2015].

Two final, important remarks:

1. Vis-a-vis quantum teleportation, one might associate the scheme to a quantum cloning machine as the final state of Bob's photon after the transformation $U$ corresponds exactly to the initial state of photon 1. However, such perspective is completely wrong as the photon 1 is destroyed during the Bell state measurement. Therefore, no qubit cloning can be achieved this way.

2. The use of the classical communication is compulsory towards communicating the Bell state measurement results. Without classical communication, the state of photon 3 will be meaningless to Bob. It also ensures the non-violation of the special relativity since there is no superluminal communication taking place between Alice and Bob. 


\subsubsection{Entanglement swapping}

Fruitful lesson learned from previous section: the quantum teleportation protocol benefits from the fact that two independent photons 1 and 2 (see Fig.2.3), i.e. sharing no common "quantum past", can be made entangled through the Bell state measurement. Genuinely, this notion can enable, not just the teleportation of the state of individual qubits, but also the entangled states. The principle of entanglement swapping (see Fig.2.4) is quite similar to the standard quantum teleportation protocol. Despite those protocols being completely similar, a thorough discussion involving the mathematical framework and experimental setup on entanglement swapping in quantum relay configuration will be presented later in Chapter 9 of this manuscript. Experimental realizations of entanglement swapping have been carried out, among others, using polarization qubits [Pan et al. 1998, Yang et al. 2006, Kaltenbaek et al. 2009, Jin et al. 2015], as well as time-bin qubits [De Riedmatten et al. 2005, Takesue $\&$ Miquel 2009].

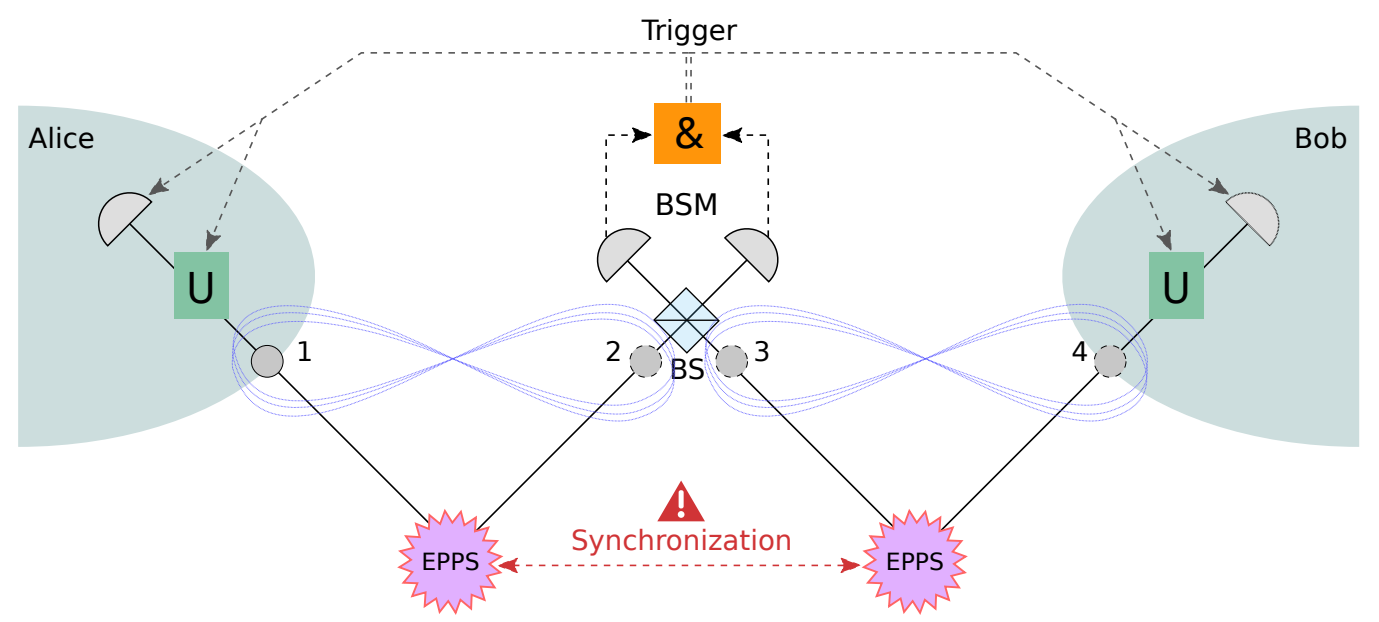

Figure 2.4: Entanglement swapping in the quantum relay configuration involves the use of two entangled photon pair sources (EPPS). The two inner photons, namely photons 2 and 3 are directed toward the Bell state measurement apparatus. Consequently, these two photons are now projected onto one of the maximally entangled Bell states. Such measurement will produce a trigger signal which alerts Alice and Bob on when to open their respective detectors and on the choice of unitary transformation $U$ enabling the reconstruction of the intended correlation between photons 1 and 4. As we know already, the BSM produces probabilistic outcomes, making the trigger signal mandatory, granting, by the way, special relativity. Remarkably, this condition holds true for all quantum protocols involving any type of teleportation.

As a side note, in any "teleportation" protocol, whether it be the standard teleportation 
or even the entanglement swapping, the synchronization between photon sources (single or entangled) represents an actual challenge for most experimentalists. This synchronization issue depends heavily on the working regime of the employed sources such that one can tackle this issue by operating either in continuous or pulsed regime. Nonetheless, both solutions have their specific advantages and disadvantages, as addressed in [Aboussouan et al. 2010]. Yet, one can always define a trade-off between the two. We will also discuss this profoundly again in Chapter 9 . 
Part II

Polarization entanglement generation at telecom wavelength 

Chapter 3

\section{Advances in polarization entangled}

\section{photon pair generation}

\section{Contents}

3.1 Advances in polarization entanglement via SPDC . . . . . . . 61

3.2 The relevance of polarization entanglement $\ldots \ldots \ldots \ldots$

3.3 A quick recap on generating photon pairs via SPDC in PPLN/W 67

It is fair to say that the challenge posed by the EPR trio [Einstein et al. 1935] has somehow ignited the pursuit of photonic polarization entanglement sources. As a matter of fact, some of the very first attempts to violate the Bell's inequality [Bell 1964] involves the exploitation of polarization entangled photon pairs generated through the implementation of atomic-cascade decays [Freedman \& Clauser 1972, Clauser \& Shimony 1978, Aspect et al. 1981, Aspect et al. 1982a, Aspect et al. 1982b]. With such a strategy, relatively "bulky" sources are implemented in order to obtain polarization entangled photons emitted by single calcium ions via a two-photon cascade process. By "bulky", we literally mean that such sources, entirely, can easily dominate the available space offered by a standard lab room.

\subsection{Advances in polarization entanglement via SPDC}

Eventually, more and more compact entangled photon pair sources were introduced thanks to the exploitation of spontaneous parametric down conversion (SPDC) in bulk non-linear crystals. Within the framework of photonic polarization entanglement, pioneering works were mostly based on the exploitation of the so-called type-II phase matching of SPDC 
process. In 1993, T. E. Kiess and his co-workers demonstrated for the first time the violation of Bell inequality with 22 standard deviations [Kiess et al. 1993]. In this experiment, cross-polarized photon pairs at $702.2 \mathrm{~nm}$ are generated thanks to the use of a $351.1 \mathrm{~nm}$ laser light pumping a bulk, type-II beta barium borate (BBO) crystal. The entanglement postselection is then performed. Vis-a-vis the implementation, it is much easier to realize this type of source compared to the atomic-cascade decays. More recently, the similar idea of post-selecting the entangled state from a type-II generated photon pairs has been reported in [Kuklewicz et al. 2006]. However, a major disadvantage concerning the entanglement post-selection is the reduction of the achievable experimental rate by $50 \%$. However, it was in 1995 that P. G. Kwiat and his co-workers first demonstrated a "true" photonic polarization entanglement source that eliminates the need of any post-selection procedure. In this work, again, a laser light at $351 \mathrm{~nm}$ is incident on a BBO crystal (see Fig.3.1). Consequently, cross-polarized photon pairs at $702 \mathrm{~nm}$ are generated via the type-II SPDC process, but, in contrast to [Kiess et al. 1993], the pairs are produced in two distinct emission cones. In this case, the incident angle of the pump light on the BBO crystal has to be properly adjusted such that the two emission cones can be made overlapped. Horizontally polarized photons can be found in the extraordinary cone while vertically polarized ones are found in the ordinary cone. By collecting photons only at the intersection points, say $a$ and $b$ (see Fig.3.1), it is impossible to know the polarization mode associated with a photon that belongs to that spatial mode. Therefore, the entangled state of $\left|\Psi^{+}\right\rangle_{a b}=\frac{1}{\sqrt{2}}\left(|H\rangle_{a}|V\rangle_{b}+|V\rangle_{a}|H\rangle_{b}\right)$ is obtained. In the quantum photonics community, this type of polarization entanglement source is referred as the "Kwiat's source" or the "cone source". Since then, countless experiments in various branches of quantum information science have successfully benefited from such cone source. To name a few, this includes the violation of the Bell inequality [Strekalov et al. 1996, Weihs et al. 1998], the demonstration of the famous quantum teleportation protocol [Bouwmeester et al. 1997, Boschi et al. 1998], the implementation of both free-space and fiber-based quantum key distribution (QKD) protocol [Jennewein et al. 2000, Erven et al. 2008], the introduction of quantum dense coding for quantum communication [Mattle et al. 1996], as well as entanglement-based delayed-choice experiment [Kaiser et al. 2012a].

The generation of polarization entanglement is not limited only to type-II SPDC. One can also implement a suitable experimental arrangement permitting the engineering of po- 


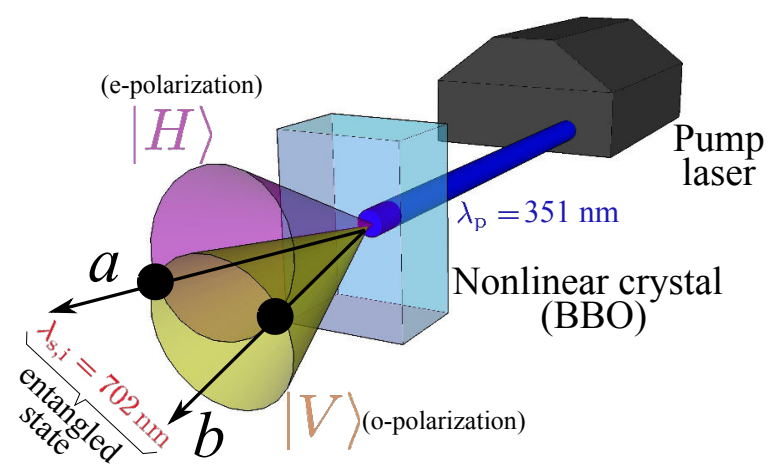

Figure 3.1: Working principle of polarization entangled photon pair sources demonstrated by P. Kwiat in 1995 [Kwiat et al. 1995].

larization entanglement from the use of either type-0 and type-I bulk crystals. For instance in [Kim et al. 2000], two type-I bulk BBO crystals are installed on each arm of a balanced Mach-Zehnder interferometer and pumped by the same laser source, resulting in the generation of $|\psi\rangle=\frac{1}{\sqrt{2}}(|2 H, 0\rangle+|0,2 V\rangle)$ states. Such states can be obtained through a proper rotation of one of the employed bulk crystal. By post-selecting the events of interest upon the action of the second beam splitter of the interferometer, an entangled state of the form $|\psi\rangle=\frac{1}{\sqrt{2}}(|H\rangle|H\rangle+|V\rangle|V\rangle)$ is obtained. However, such Mach-Zehnder interferometric solution is prone to the environmental fluctuations such that the phase inside the interferometer may vary. Even though in a free-space interferometric configuration, the effect of such disturbances is much less severe than in a fiber-based one, the phase still should be kept stable in order to observe high-quality entanglement. Therefore, the interformeter should be stabilized either passively or actively, which adds to the burden of the experimentalist. Such stabilization issue can be relaxed, for instance, by implementing a Sagnac interferometric configuration, as demonstrated by B-S. Shi and A. Tomita in 2004. In their experiment, generation of polarization entangled photon pairs is achieved through the use of a type-I bulk BBO crystal positioned in a Sagnac loop [Shi \& Tomita 2004]. As a matter of fact, both presented interferometric solutions still rely on entanglement post-selection procedures. It is interesting to note that several years before, this issue has again been addressed by Kwiat and his co-workers. In 1999, they demonstrated another "cone" photonic polarization entanglement source by stacking together two identical type-I bulk BBO crystals [Kwiat et al. 1999]. In this case, one crystal must be oriented perpen- 
dicularly to the other one in term of optical axis (see Fig.3.2). Then, when a diagonally polarized pump field is incident on the stacked crystals, an entangled state of the form $|\psi\rangle=\frac{1}{\sqrt{2}}(|H\rangle|H\rangle+|V\rangle|V\rangle)$ can be obtained automatically. As a side note, there exists a variety of methods for optimizing this particular type of source, as later proposed by their group [Rangarajan et al. 2009]. The "stack" solution for polarization entanglement has also been implemented with bulk type-0 periodically poled potassium titanyl phosphate (KTP) crystals [Steinlechner et al. 2012].

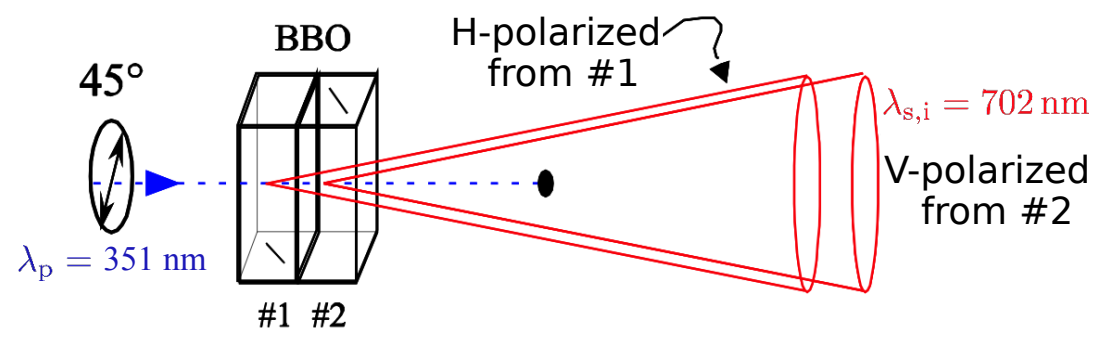

Figure 3.2: Working principle of polarization entangled photon pair sources by stacking together two identical type-I bulk BBO crystals. one crystal must be oriented perpendicularly to the other one in term of optical axis.

More recently, following past implementations for energy-time entanglement [Tanzilli et al. 2001, Sanaka et al. 2001, Tanzilli et al. 2002] (note that all mentioned realizations have relied on type-0 SPDC co-polarized photon pairs ), we have seen also the exploitation of waveguide structures embedded in or integrated of the surface of the non-linear crystal in which optical fields can strongly be confined over longer interaction lengths. This way, high field intensities are available for any desired non-linear interactions. In order to satisfy the conditions for the SPDC to take place in such structures, the quasi phase matching (QPM) is implemented. In this sense, such structures are generally known as periodically poled waveguide devices. Concerning the generation of the polarization entanglement in periodically poled waveguide-based devices, several works have been reported on the use of type-II SPDC [Fujii et al. 2007, Suhara et al. 2007, Martin et al. 2009, Martin et al. 2010, Zhong et al. 2010]. This also includes the work of this thesis presented in chapter 3 (see also [Kaiser et al. 2012b]). As has been discussed earlier, with bulk solution, polarization entanglement can also be engineered from co-polarized photon pairs generated via type-0 and type-I interactions. The same could be achieved with waveguide-based implementation. 
Notably, the same experimental principle found in [Kim et al. 2000] has been implemented using PPLN/W [Yoshizawa et al. 2003]. Interestingly, the work of Yoshizawa and his coworkers that was performed in 2003 actually represents the first ever reported polarization entanglement at the telecom wavelength of $1550 \mathrm{~nm}$. In this particular experiment, two type0 PPLN/Ws are positioned each in one arm of a Mach-Zehnder interferometer and they are pumped by the same laser source. Meanwhile in 2005, Takesue and his co-workers have benefited from an unbalanced Michelson interferometer in order to generate polarization entanglement from type-0 SPDC in a single PPLN/W [Takesue et al. 2005]. In 2007, Jiang and Tomita have once again employed a Sagnac loop configuration. Only this time, PPLN/W is employed in order to generate polarization entangled photon pairs at telecom wavelength [Jiang \& Tomita 2007]. Interesting enough, a combination of Sagnac and MachZehnder interferometers in a single experimental setup has led to a recent demonstration of three-photon polarization entanglement [Hamel et al. 2014]. Recently, serious efforts have also been made on electrically driven, photon pair generation in aluminium gallium arsenide $(\mathrm{AlGaAs})$ ridge waveguide layered structures toward generating polarization entanglement [Boitier et al. 2014, Autebert et al. 2015].

As a short note, photon pairs can also be generated in a guided-wave manner via the four-wave mixing (FWM) found in optical fibers [Fiorentino et al. 2002, Li et al. 2004, Takesue \& Inoue 2004, Dyer et al. 2009], photonic crystal fibers [Fan et al. 2005, Rarity et al. 2005, Fulconis et al. 2005] and more particularly in silicon nanophotonic devices [Sharping et al. 2006, Harada et al. 2008, Clemmen et al. 2009, Xiong et al. 2011]. Concerning polarization entanglement, several demonstrations have been reported in the literature. Good examples can be found in [Li et al. 2005, Takesue et al. 2008, Takesue et al. 2010, Almeida et al. 2011, Lv et al. 2013, Zhou et al. 2013]. However, the performance of FWM sources are generally affected by Raman scattering and multi-photon absorption.

\subsection{The relevance of polarization entanglement}

Indeed, polarization entanglement represents the most natural and accessible entanglement observables there is. Easy manipulation and analysis of photonic polarization state have made it the most popular choice toward studying fundamental quantum effects in the early 
days of quantum physics. Sure, various entanglement sources based on other photonic observables have been demonstrated over time. Particularly, since the introduction QKD in 1983, we have seen highlights from a series of novel experiments exploiting, for instance, the "time" correlation in the form of energy-time [Franson 1989, Kwiat et al. 1993, Tanzilli et al. 2002], as well as time-bin [Tanzilli et al. 2002, Thew et al. 2002, Dynes et al. 2009] entanglement in order to quantumly communicate over long distances. Facing to such situation, it is not possible for someone to bring up the following question: is the photonic polarization entanglement still relevant in this modern day of quantum communication where long distance quantum link based on optical fibers is considered as the holy grail? Or, is it only suitable for fundamental studies of the quantum mechanics?

It is an undeniable fact that in optical fibers, photons suffer from the polarization drifts due to birefringence fluctuations induced by the enviromental changes. However, active compensation of fiber birefringence fluctuations can be now be adopted to ensure an overall polarization stability [Chen et al. 2007, Xavier et al. 2009, Chen et al. 2009]. Moreover, the not so good perspective toward polarization entanglement has now been shifted thanks to a breakthrough article published in 2001 entitled Long-distance quantum communication with atomic ensembles and linear optics [Duan et al. 2001]. Through this theoretical proposal, it is understood that the achievable distance of QKD can be considerably increased as per following. While creating a single atomic excitation, an atomic ensemble can emit a single photon. Similarly, the same behavior can be set-up for a second atomic ensemble. If these emitted single photons are subjected to a projective measurement, these two distanced atomic ensembles can be made entangled. In this case, proper entanglement generation is expected when the photons are projected onto a polarization entangled state. Intuitively, the same can be observed when considering two separate atomic ensembles share a pair of polarization entangled photons. Consequently, both ensembles can also be entangled, i.e. the entanglement is stored in the ensembles. By doing so, these separated ensembles can act as quantum memory devices, aiding the synchronization between separated polarization entangled photon pair sources for entanglement swapping applications, leading to more efficient overall network [Chanelière et al. 2005a, Sangouard et al. 2011, Kaiser et al. 2013]. In order to ensure the correct functionality of this method, the single photon spectral bandwidth should be adapted to the absorption bandwidth of the implemented 
quantum memories. Interestingly, this becomes one of the headlines to this thesis where a photonic polarization entanglement source whose the emitted photon spectral bandwidth is compatible with certain quantum memory devices is demonstrated.

Vis-a-vis the relevance toward demonstrating high-quality photonic entanglement sources, two of such sources based on both type-II and type-0 SPDC in PPLN/W are highlighted in this thesis. With the type-II source, we want to show how relatively compact a polarization entanglement source for quantum communication applications at telecom wavelength could be [Kaiser et al. 2012b]. Meanwhile, with a type-0 source, we aim to demonstrate the versatility in term of single photon spectral bandwidth, to be adapted to broad variety of quantum applications. To kick off, we first briefly describe the photon pair generation via SPDC [Kaiser et al. 2013].

\subsection{A quick recap on generating photon pairs via SPDC in $\mathrm{PPLN} / \mathrm{W}$}

Spontaneous parametric down conversion (SPDC) process in nonlinear crystals has a good reputation of being the most convenient tool for generating photonic entanglement. Being a three-wave mixing process, SPDC is responsible for giving birth, with a certain probability, to a a pair of lower energy signal (s) and idler (i) photons when a high energy pump photon ( $\mathrm{p}$ ) is passing through a birefringent crystal featuring $\chi^{(2)}$ non-linearity such as lithium niobate (LiNbO3), potassium titanyl phosphate (KTP) and beta barium borate (BBO). For all the three photon fields, conservation of energy and momentum must be obeyed such that

$$
\begin{array}{r}
\omega_{p}=\omega_{s}+\omega_{i}, \\
\vec{k}_{p}=\vec{k}_{s}+\vec{k}_{i},
\end{array}
$$

where $\omega$ denotes the photon angular frequency. Meanwhile, $\vec{k}$ depicts the photon momentum where

$$
\vec{k}_{x}=\frac{2 \pi n_{x}}{\lambda_{x}} \vec{u} \text { with } x \in\{p, s, i\}
$$


Here, $n$ is the non-linear medium refractive index, $\lambda$ represents the photon vacuum wavelength and $\vec{u}$ is the normalized vector along the light propagation axis.

In the early days, photon pair generation via SPDC has been demonstrated in many quantum experiments, particularly, through the use of bulk crystals [Ghosh \& Mandel 1987, Hong et al. 1987, Ou \& Mandel 1988, Shih \& Alley 1988, Kiess et al. 1993]. In this case, the condition of the Eq.(3.2), i.e. the phase matching condition is mostly fulfilled via critical phase matching (CPM), also known as angle phase matching. As being circumstanced by Eq.(3.3), CPM is achieved by selecting the good emission angle of a pump field at particular wavelength to the optical axis of the bulk crystal. However, through CPM, it is really difficult to maintain high field intensities over more than a few $\mathrm{mm}$, thus reducing the interaction lengths. To circumvent such issue, the employed crystals can be put inside an optical cavity [Kuklewicz et al. 2006, Neergaard-Nielsen et al. 2007].

Another pertinent method to obtain the required high field intensities in non-linear crystals is to confine light along a waveguide structure which is buried in or integrated of the surface of the material itself. By taking advantage of such structures, light can be strongly confined over much longer lengths, on the order of a few $\mathrm{cm}$. Consequently, unprecedented SPDC efficiency can be achieved. However, the conservation of momentum is not naturally satisfied a waveguide structure. It is due to the dispersion of the material itself that the phase of the interacting fields are potentially mismatched. Consequently, after a short distance in the crystal, the signal and idler fields interfere destructively. In the case of bulk crystals, the phase matching is fulfilled by compensating the dispersion through the exploitation of the crystal natural birefringence [Giordmaine 1962, Maker et al. 1962].

As an alternative, the so-called quasi phase matching (QPM) can be implemented in order to prevent the phase shift between the interacting fields, thus satisfies the conditions for SPDC to take place in a waveguide structure. The QPM was early discussed in [Armstrong et al. 1962] and [Franken \& Ward 1963]. Thanks to QPM, the non-linear $\chi^{(2)}$ coefficient of the material is periodically inverted along the light propagation axis and thus compensates any potential phase mismatches such that the original phase matching condition of Eq.(3.2) is transformed into

$$
\vec{k}_{p}=\vec{k}_{s}+\vec{k}_{i}+\frac{2 \pi}{\Lambda} \cdot \vec{u}
$$


where $\Lambda$ denotes the poling period and $\vec{u}$ is the normalized light propagation vector. In this case, an appropriate choice of $\Lambda$ permits obtaining an artificial phase matching condition for a desired combination of pump, signal and idler wavelengths within the transparency window of the crystal (considering no technological constraint). Moreover, thanks to the high dependency of crystal birefringence to the temperature, a fine tuning of phase matching can be performed by simply heating up or cooling down the crystal. Note that in terms of the transparency window, LiNbO3, KTP, and BBO materials are highly transparent over wavelength ranges of 350-5200 nm, 350-2700 nm, and 190-3300 nm, respectively.

Concerning SPDC in LiNbO3, the highest non-linear coefficient $d_{33} \approx 30 \mathrm{pm} / \mathrm{V}$ used for type-0 interaction can be exploited through the QPM. Such a value is generally six times higher than the $d_{31}$ coefficient of type-I interaction used in bulk crystals. Therefore LiNbO3 can exhibit high conversion efficiency in the order of up to $10^{-5}$, leading to high generation rate of photon pairs at a relatively low pump power. As a side note, the use of bulk crystal configuration leads to conversion efficiency in the order of $10^{-12}-10^{-10}$. In order to compensate the lower conversion efficiency for high photon pair generation rate, higher pump powers are therefore required.

Historically speaking, the persuasion of entangled photon pair source via SPDC is not the main reason why QPM is heavily studied. Rather, the motivation is driven by the inverse process of SPDC which is the second harmonic generation (SHG) [Fejer et al. 1992]. Nevertheless, the exploitation QPM to fulfill the conditions for SPDC in, for instance, LiNbO3 has its own place in the world of quantum information science. It was in the early of $21^{\text {st }}$ century that we saw some of the very first demonstration of quantum entanglement concerning photon pairs produced by QPM-based sources [Tanzilli et al. 2001, Sanaka et al. 2001, Tanzilli et al. 2002].

Until today, most of the experiments involving the generation of photon pairs in periodically poled crystals basically exploit the three main interaction types which relate to the polarization states of the pump, signal and idler photons (see Tab.3.1). For this thesis, the exploitation of type- 0 and type-II SPDC interactions in PPLN/Ws toward generating photon pairs at telecom wavelength are highlighted. 
Table 3.1: The most exploited interaction types of SPDC for generating photon pair in PPLN/W. In this thesis, the exploitation of type-0 and type-II SPDC interactions in a PPLN/W are highlighted. Please note that the fabrication techniques concerning PPLN/W is not this thesis' aim. For further insight into the matter, information contained in [Fouchet et al. 1987], [Sohler 1989], [Chanvillard et al. 2000] might be of interest to certain readers.

\begin{tabular}{llll}
\hline Interaction type & type-0 & type-I & type-II \\
\hline Polarization & $|V\rangle_{p} \rightarrow|V\rangle_{s}|V\rangle_{i}$ & $|V\rangle_{p} \rightarrow|H\rangle_{s}|H\rangle_{i}$ & $|H\rangle_{p} \rightarrow|H\rangle_{s}|V\rangle_{i}$ \\
$\chi^{(2)}$ coeff. of LiNbO3 & $d_{33} \approx 30 \mathrm{pm} / \mathrm{V}$ & $d_{31} \approx-5 \mathrm{pm} / \mathrm{V}$ & $d_{24} \approx 10 \mathrm{pm} / \mathrm{V}$ \\
$\eta_{S P D C}$ in & $10^{-6}-10^{-5}$ & $10^{-7}$ & $10^{-9}$ \\
$\Delta \lambda$ at $1550 \mathrm{~nm}$ & $20-100 \mathrm{~nm}$ & $20-100 \mathrm{~nm}$ & $0.8-3 \mathrm{~nm}$ \\
$\Delta \nu$ at $1550 \mathrm{~nm}$ & $2.5-12.5 \mathrm{THz}$ & $2.5-12.5 \mathrm{THz}$ & $0.1-0.375 \mathrm{THz}$ \\
Natural entanglement & Energy-time & Energy-time & Polarization \\
\hline
\end{tabular}




\section{Compact polarization entanglement}

\section{source based on a type-II PPLN/W}

\section{Contents}

4.1 Motivation: quantum key distribution . . . . . . . . . . 71

4.2 Compact polarization entanglement generator: the experiment . . 72

4.2 .1 Photon pair generation $\ldots \ldots \ldots \ldots \ldots$

4.2.2 Temporal walk-off compensation _ . . . . . . . . . . . 75

4.2.3 Low-loss and deterministic photon pair separation . . . . . . . . . 82

4.2.4 Entanglement quality measurement . . . . . . . . . . . . . . . . 84

4.2 .5 Entangled state symmetry manipulation . . . . . . . . . . . . . 89

4.2 .6 Source's performances . . . . . . . . . . . . . . . . . . . . . . . . . . 93

4.3 Conclusion and perspectives $\ldots \ldots \ldots \ldots \ldots \ldots$

Some results presented in this chapter were published in F. Kaiser, A. Issautier, L. A. Ngah et al., High-quality polarization entanglement state preparation and manipulation in standard telecommunication channels, New Journal of Physics, Volume 14, Number 8, pages 085015, August 2012.

\subsection{Motivation: quantum key distribution}

Photonic entanglement is a key resource for quantum communication protocols, among which today's main application is quantum key distribution (QKD) in standard dense wavelength division multiplexing (DWDM) ITU channels [Qi et al. 2010b, Yoshino et al. 2012,Patel et al. 2014]. Producing entangled photons within the telecom C-band of wavelengths 
(1530-1565 nm) enables implementing relatively long-distance quantum links, thanks to lowloss optical fibers and high performance standard components. One of the most natural and accessible entanglement observables is the polarization state of the photons.

In this chapter, we discuss a simple and compact, yet practical approach for generating high-quality polarization entanglement in a fully guided-wave fashion. We take advantage of a type-II photon pair generator. Moreover, both deterministic pair separation in two adjacent telecommunication channels and walk-off compensation are achieved using standard fiber components. Two-photon interference experiments are carried out both for precisely determining the length of the fiber compensation stage, and for manipulating the produced state between bosonic $\left|\Psi^{+}\right\rangle$and fermionic $\left|\Psi^{-}\right\rangle$symmetries [Matthews et al. 2013]. Eventually, we perform a Bell inequality test with the $\left|\Psi^{+}\right\rangle$state to quantitatively demonstrate the relevance of both our approach and results.

\subsection{Compact polarization entanglement generator: the exper- iment}

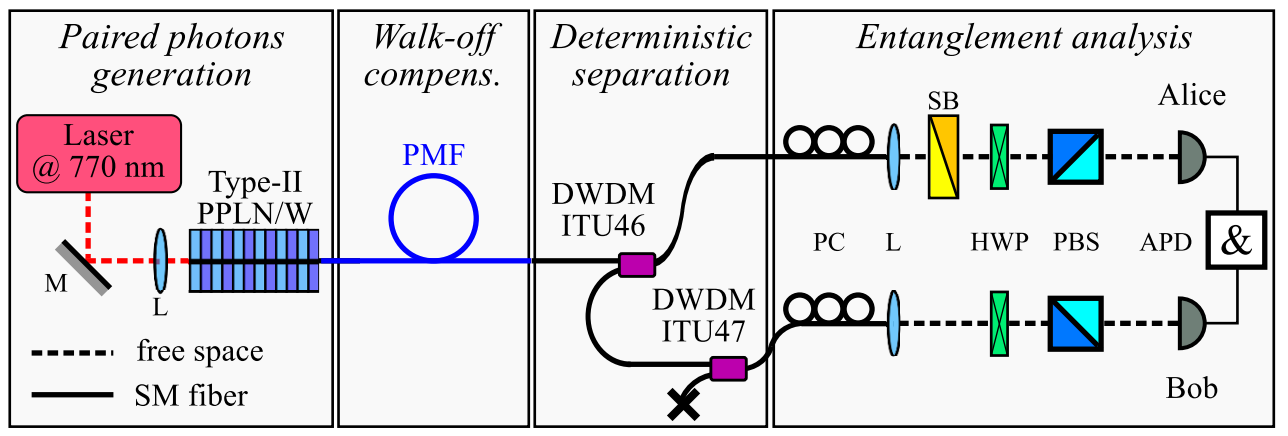

Figure 4.1: Experimental setup for generating polarization entangled photons at $1540 \mathrm{~nm}$ taking advantage of a type-II spontaneous parametric down conversion (SPDC) in a periodically poled lithium niobate waveguide $(\mathrm{PPLN} / \mathrm{W})$ pumped by a $770 \mathrm{~nm}$ laser. A polarization maintaining fiber (PMF) is used as the birefringence compensator and a set of two DWDMs separates the photons deterministically into the standard 46 and 47 ITU channels. A standard Bell inequality apparatus measures the entanglement quality. On Alice's side, a Soleil-Babinet phase compensator (SB) permits adjusting the phase of the entangled state. L: lens, PC: fiber polarization controller, HWP: half wave-plate, PBS: polarising beam splitter, \& : coincidence counter.

The overall experimental setup of the compact polarization entanglement source based 
on a type-II PPLN/W is depicted in Fig.4.1 where it can be broken into several categories or segments which are:

- The generation of photon pairs;

- The walk-off compensation;

- The deterministic separation of photon pairs;

- The entanglement analysis.

As a matter of fact, this section is organized accordingly to the "development flow" of the source. Note that any measurement outcomes concerning a specific segment are directly discussed.

\subsubsection{Photon pair generation}

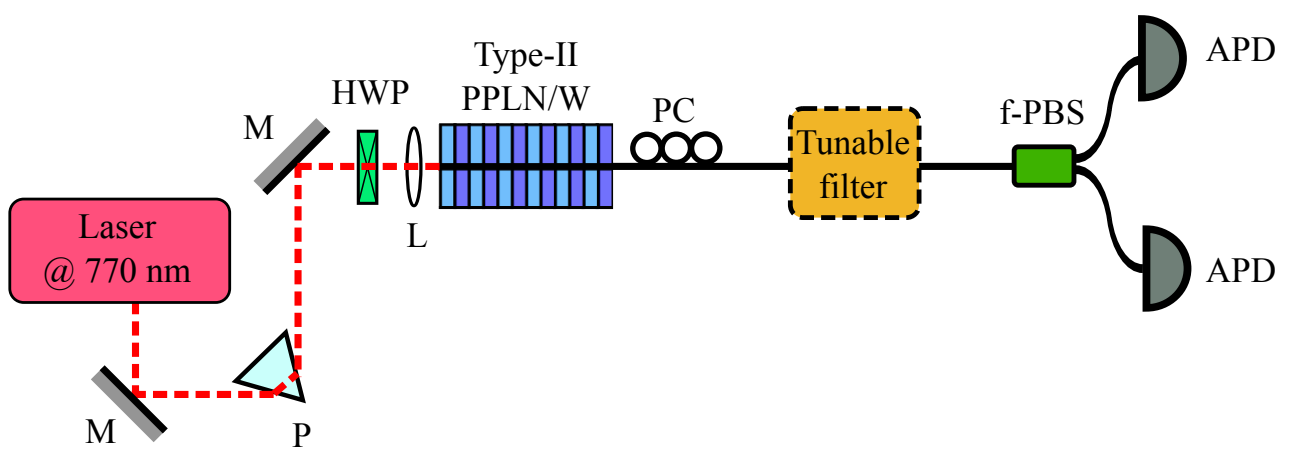

Figure 4.2: Outline of the experimental setup used for observing the polarization-dependent, type-II SPDC emission spectrum. The pump light at $770 \mathrm{~nm}$ is first filtered by a prism (P) in order to get rid of any unwanted infrared emission. The pump light is then horizontally polarized by a half-wave plate (HWP) before being injected into a type-II PPLN/W. The generated photon pairs are collected by a single mode fiber and sent toward a polarization controller (PC) followed by a tunable filter. Next to the filter, a fiber polarizing beam splitter is employed to split the incoming horizontal and vertical polarized photons. Eventually, the separated photons are detected by two avalanche photo-diodes (APD).

We first begin by describing, experimentally, the photon pair generator itself. Let us consider the experimental setup shown in Fig.4.2. Here, a continuous-wave (CW) laser source (Coherent MBR110) is employed as the pump light. The laser pump light at $770 \mathrm{~nm}$ is first incident onto a prism so as to filter out any undesired infrared emission produced 
by the laser. Consequently, only NIR light is allowed to be injected in a $3.6 \mathrm{~cm}$ long typeII periodically poled lithium niobate waveguide $(\mathrm{PPLN} / \mathrm{W})^{1}$. Through the SPDC nonlinear interaction in such a waveguide, cross-polarized photon pairs $|H\rangle_{s}|V\rangle_{i}$ around the degenerate wavelength of $1540.2 \mathrm{~nm}$ are generated. One should note, however, that the input light should be horizontally polarized so as to fulfill the QPM condition. This is simply done by employing a rotating half wave plate just before the PPLN/W.

Already, we can proceed to the first, yet important, characterization of the waveguide which consists of verifying the spectral indistinguishability of the produced polarizationdependent SPDC emission spectrum. To this end, the generated photon pairs are collected by butt-coupling a single mode fiber (SMF) to the PPLN/W. The collected photon pairs are then directed toward a tunable optical filter (Yenista XTM-50). The transmission bandwidth of such filter is fixed at $80 \mathrm{pm}$ which is small enough to provide sufficient resolution to the measurement as it is much narrower than the expected emission bandwidth. Following the spectral filtering, the pairs are then sent to a fiber polarizing beam splitter (f-PBS) where the transmitted and reflected photons are then detected by two free-running single photon detectors (IDQ 220). Eventually, the corresponding count rates are recorded as a function of the wavelength given by the tunable filter. In order to optimize the detection, the global polarization drift experienced by both polarization modes across the experimental setup is compensated by a fiber polarization controller (PC).

As shown in Fig.4.3, rather narrow SPDC emission spectra for both polarization modes centered at the wavelength of $1540.2 \mathrm{~nm}$ are observed. Such a phase matching condition is obtained through the use of a temperature stabilized PPLN $/ \mathrm{W}$ at $110.2^{\circ} \mathrm{C}$, having a width of $6 \mu \mathrm{m}$ and a poling period of $9 \mu \mathrm{m}$. For both spectra, the full width at half maximum (FWHM) is measured to be $0.85 \mathrm{~nm}$. For most part of the spectrum, potential mismatch in term of the wavelength, notably in the outer side peak regions, is observed. Remarkably, this represents a major concern as any mismatch of the two polarization modes (aside the polarization itself) will degrade the quality of the expected entanglement [Martin et al. 2010]. Basically, any measurement that reveals the wavelength can indeed tell us in what polarization state the photon is actually in. However, good overlapping regions can be conveniently

\footnotetext{
${ }^{1}$ The sample is designed and fabricated at the University of Paderborn in Germany. Dr. Harald Herrmann and Prof. Dr. Wolfgang Sohler are warmly acknowledged for their collaboration.
} 


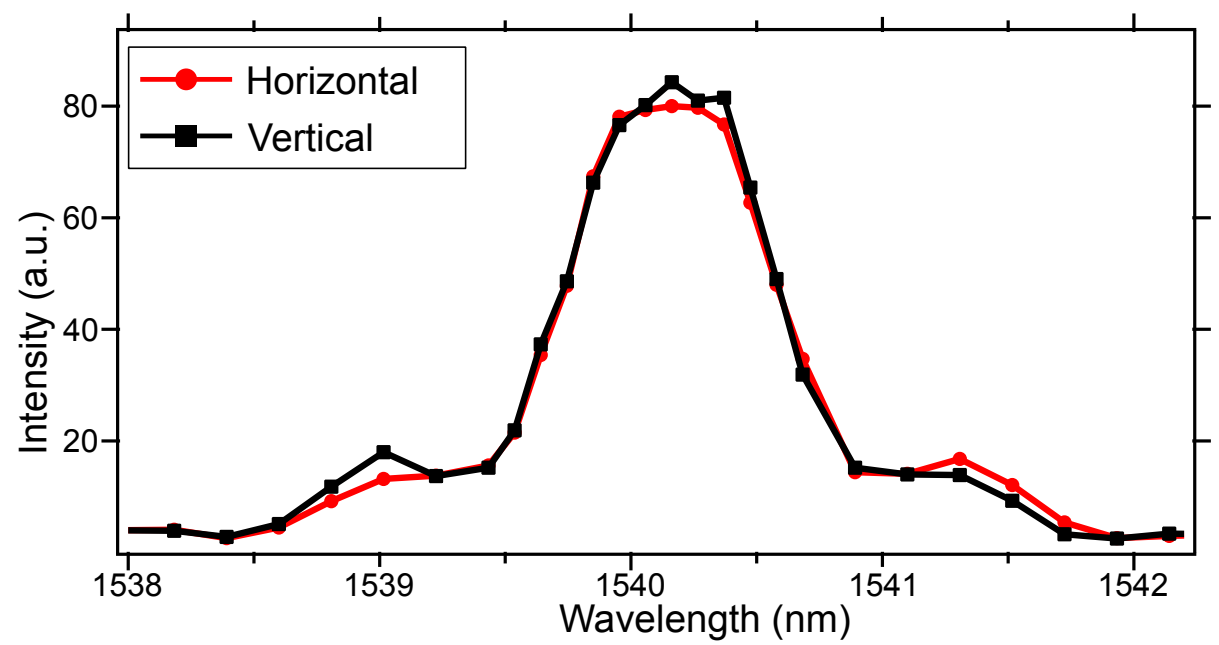

Figure 4.3: Polarization-dependent SPDC emission spectrums for both polarization modes. For both emission modes, the full width at half maximum (FWHM) is measured to be around $0.85 \mathrm{~nm}$. The phase matching conditions are obtained through the use of a PPLN $/ \mathrm{W}$ at having a sidth of $6 \mu \mathrm{m}$ and a poling period of $9 \mu \mathrm{m}$. The PPLN/W is also temperature stabilized at $110.2^{\circ} \mathrm{C}$. The two polarization modes show a near-perfect overlap with the exception in the outer side peak regions.

distilled from the overall spectrum through a suitable spectral filtering. For instance, one can simply employ a standard band-pass filter centered a the degenerate wavelength of the produced photon pair $(1540.2 \mathrm{~nm})$, provided the acceptance bandwidth is adequately chosen. Perhaps even more intriguingly, by cleverly arranging the filtering scheme, we can kill two birds with one stone. It means that, first, such scheme can ensure high-quality entanglement by rejecting the unwanted photon on the side peaks and secondly, it can separate deterministically the photon pairs at low level of losses.

\subsubsection{Temporal walk-off compensation}

As has been discussed previously, any physical parameter that could lead to some distinguishability between the two polarization modes would indeed degrade the quality of entanglement. In this case, the temporal distinguishability, i.e. the temporal walk-off caused by the waveguide birefringence, is no exception. During the photon pair creation process, the birefringence inherent to the PPLN/W forces one photon to exit the waveguide slightly earlier compared to the other one. Theoretically, this yields a time delay between the two 
$|H\rangle$ and $|V\rangle$ photons of

$$
\Delta t=\frac{L \cdot \Delta n}{2 c}
$$

where $L=0.036 \mathrm{~m}$ is the sample length, $c=299792458 \mathrm{~ms}^{-1}$ is the speed of light in vacuum, and $\Delta n$ represents the difference between the ordinary and the extraordinary refractive indices. At the telecom wavelength of $1540 \mathrm{~nm}$, the ordinary and extraordinary refractive indices of LiNbO3 are 2.2115 and 2.1378, respectively. This leads to $\Delta n=0.0737$. The factor $\frac{1}{2}$ arises from the fact that in average, the photon pairs are generated at half the length of the sample. By plugging in all the required parameters in Eq.(4.1), $\Delta t$ for our sample is computed to be $\sim 4.3 \mathrm{ps}$. This value is proved to be comparable to the emission coherence time of the PPLN $/ \mathrm{W}$ itself $(\Delta \lambda=0.85 \mathrm{~nm} \rightarrow \Delta \tau=4.11 \mathrm{ps})$. Consequently, this temporal mismatch cannot be considered negligible. Naturally, one would consider to apply narrow filtering on the generated photons so as to greatly increase the photons' coherence time [Piro et al. 2009]. If the photons' coherence time is much greater than $\Delta t$, the constraint on the temporal walk-off can be completely relaxed. However, the price to pay for increasing the coherence time is in a reduced available photon pair rate. On the other hand, one could also apply the adjustable birefringence compensator setup [Martin et al. 2010] as illustrated in Fig.4.4 in order to erase such temporal mismatch between two polarization modes.

However, both solutions depicted above share the same drawback which is the acquisition of unwanted additional propagation losses. In this regard, it is the aim of this chapter to actually introduce an essentially loss-free, temporal walk-off compensation technique. Our method simply involves the use of standard polarization maintaining fiber (PMF). This is made possible by employing a PMF showing an opposite birefringence sign compared to that of PPLN/W. The question is how long should the PMF be in order to erase the $\Delta t$ contribution inherent to our sample.

Experimentally, the delay between two different polarization modes of incoming photons $|H\rangle$ and $|V\rangle$, which represents the natural birefringence of our sample, can be conveniently estimated through a two-photon interference experiment. The quantum effect of two-photon interference has long been used in HOM dip type experiments such that $100 \%$ dip visibility is expected when two indistinguishable photons are simultaneously sent to a beam splitter 


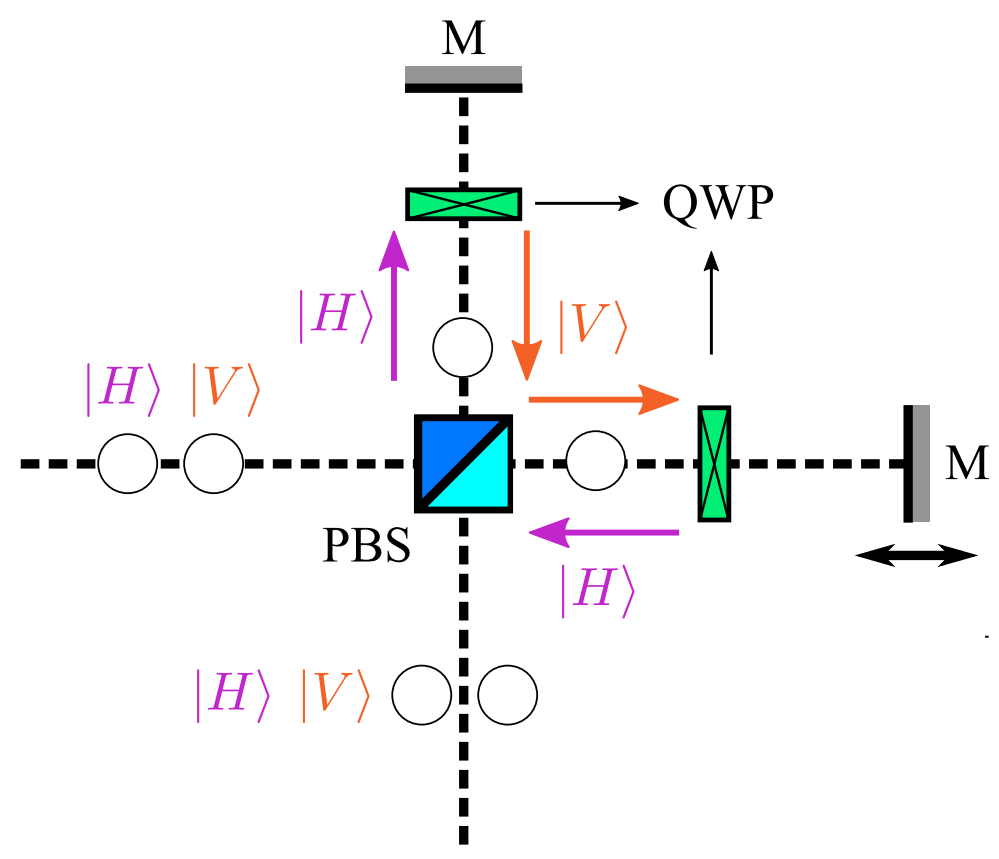

Figure 4.4: Adjustable birefringence compensator. The experimental setup is very similar to that Michelson interferometer. The principle of this birefringence compensator is very simple. The incoming cross-polarized photon pair is first separated by a polarizing beam splitter (PBS). In the path of each separated photon, a quarter-wave plate (QWP) and a mirror $(\mathrm{M})$ are employed. Each paired photon propagates twice through the respective QWP fixed at $45^{\circ}$, resulting in the rotation in its polarization state by $90^{\circ}$. Eventually, the paired photons leave the PBS through the same output. In order to erase the temporal walk-off induced by the PPLN/W natural birefringence, the path of the initial $|V\rangle$ photon needs to be made longer. A precise scanning of this parameter can be performed with the help of, for instance, a motorized mirror.

[Hong et al. 1987]. In essence, this type of experiment requires the use of a delay scanner so that the arrival time of one photon on the beam splitter can be easily adjusted relatively to the other. Consequently, any delay between these two photons can be associated with the position of the delay scanner. Inspired by such technique, we take advantage of the adjustable birefringence compensator depicted in Fig.4.4 as a polarization-dependent delay scanner apparatus. Eventually, we end up in a experimental configuration illustrated in Fig.4.5.

Generally, information from two different cases of two-photon interference measurement are required toward estimating correctly the length of PMF fiber so as to erase the temporal walk-off induced by the PPLN/W. The first case corresponds to the natural case where the temporal walk-off across the whole setup is defined only by the PPLN/W birefringence 
(a) fs pulsed laser

(b) PPLN/W + SMF

(c) $P P L N / W+P M F$
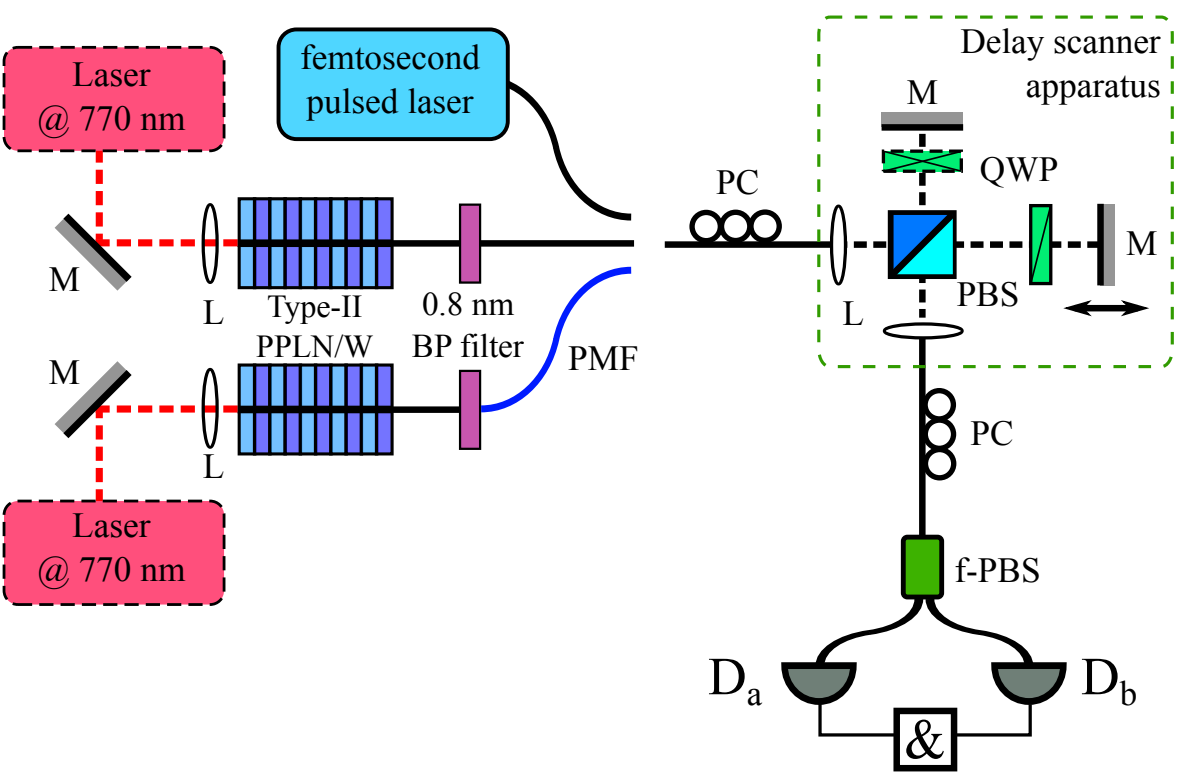

Figure 4.5: Multiple measurements toward estimating the correct length of the the polarization maintaining fiber (PMF) for temporal walk-off compensation. (a): Experimental arrangement to calibrate the delay scanner apparatus. Here, a femtosecond pulsed laser $(\lambda=\sim 1552 \mathrm{~nm})$ is employed as light source. Through a first-order interference measurement, the zero delay position of the delay scanner apparatus concerning both polarization modes of the incoming photons can be inferred. (b): The first two-photon interference measurement where the resulting outcome reflects the natural case where the temporal walk-off across the whole setup is defined only by the PPLN/W birefringence. In this measurement, the actual generated photon pairs by the type-II PPLN/W are used and only single mode fibers (SMF) are employed throughout the setup. (c): The second two-photon interference measurement. In this case, the outcome reflects the combined birefringence of both the $\mathrm{PPLN} / \mathrm{W}$ and the chosen compensator being the polarization maintaining fiber (PMF). As a side note, the principle of the delay scanner apparatus is exactly the same as the one explained in Fig.4.4.

(Fig.4.5 (b)). Meanwhile, the second case represents the combined birefringence of both the PPLN/W and the chosen compensator (PMF) (Fig.4.5 (c)). However, it is imperative to first calibrate the employed delay scanner (Fig.4.5 (a)) through a first-order interference experiment to know exactly the zero delay position of the delay scanner apparatus concerning both polarization modes of the incoming photons.

The calibration of the delay scanner involves the use of a femtosecond pulsed laser as the photon source (Calmar Mendocino). Here, optical pulses at $\sim 1552 \mathrm{~nm}$ from such laser 
is polarized diagonally using a polarization controller $(\mathrm{PC})$ such that it reads

$$
|\psi\rangle_{\text {calib }}=\frac{1}{\sqrt{2}}(|H\rangle+|V\rangle)
$$

Diagonally polarized photons are injected into the polarization-dependent delay scanner apparatus for which the following quantum state is found at the output:

$$
|\psi\rangle_{\text {calib }}=\frac{1}{\sqrt{2}}\left(|H, 0\rangle+e^{i \phi_{M}}\left|V, \Delta t_{M}\right\rangle\right)
$$

where $\Delta t_{M}$ depicts the delay induced by the movable mirror affecting the $|V\rangle$ photon and $\phi_{M}$ is the acquired phase directly related to $\Delta t_{M}$. The photons are then rotated by $45^{\circ}$ using the second PC and after the fiber polarizing beam splitter, the photon is found in the state of

$$
|\psi\rangle_{c a l i b}=\frac{1}{\sqrt{2}}\left(|H, 0\rangle_{a}+|V, 0\rangle_{b}+e^{i \phi_{M}}\left|V, \Delta t_{M}\right\rangle_{b}-e^{i \phi_{M}}\left|H, \Delta t_{M}\right\rangle_{a}\right) .
$$

Here, the subscript $a$ and $b$ represent the path chosen by the particular photon for which it will be eventually detected either by detector $\mathrm{D}_{a}$ or $\mathrm{D}_{b}$. Consequently, the detection probabilities concerning both detectors $\mathrm{D}_{a}$ or $\mathrm{D}_{b}$ are given by

$$
P_{\text {calib }, \mathrm{D}_{a}}=\frac{1}{2}\left(1+e^{-\frac{4 \cdot \ln (2) \Delta t_{M}^{2}}{\tau_{c o h}^{2}}} \cdot \sin ^{2} \frac{\phi_{M}}{2}\right) ; \quad P_{\text {calib }, \mathrm{D}_{b}}=1-P_{\text {calib }, \mathrm{D}_{a}}
$$

where $\tau_{c o h}$ denotes the coherence time of the employed calibration laser. Eq. (4.5) suggests that the oscillation in the output intensity depends on the phase $\phi_{M}$ and one can straightforwardly deduce that when the delay between both polarization modes $|H\rangle$ and $|V\rangle$ is equal to zero $\left(\Delta t_{M}=0\right)$, the oscillation reaches its maximum. As for the real measurement, the recorded outcome is shown in Fig.4.6 where the intensity in detector $\mathrm{D}_{a}$ is obtained as a function of the position of the delay scanner.

Now, we are interested in the first case of the two-photon interference experiment. From the outcome of such measurement, the experimental value of $\Delta t$ due to natural PPLN/W birefringence can be inferred. For this, we make use of cross-polarized photon pairs generated by the type-II PPLN/W. The generated photon pairs are collected using a standard 


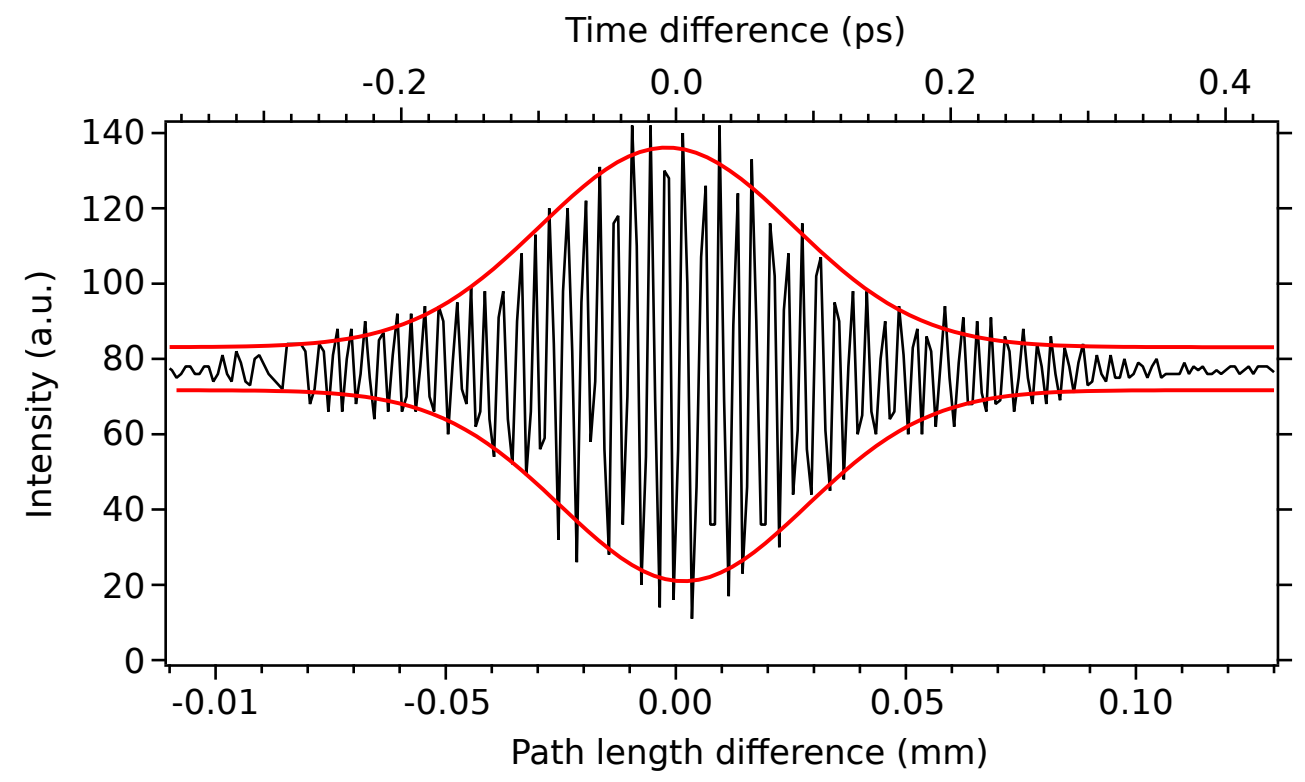

Figure 4.6: The outcome from the delay scanner apparatus calibration via a first-order interference measurement. The plotted data represents the intensity recorded by the employed detector as a function of the movable mirror's position of the scanner apparatus. The black curve originates from the actual experimental data while the red curves are the result of a Gaussian fitting function. The reference zero position of the delay scanner apparatus is found at $2 \mu \mathrm{m}$.

single mode fiber (SMF), thus the following quantum state is obtained:

$$
|\psi\rangle_{S M F}=|H, 0\rangle\left|V, \Delta t_{S M F}\right\rangle .
$$

Here, $\Delta t_{S M F}$ denotes the temporal delay between both polarization modes which is solely defined by the natural PPLN/W birefringence. Both photons are then directed toward the delay scanner and the state at the output of such scanner reads

$$
|\psi\rangle_{S M F}=e^{i \phi_{M}}|H, 0\rangle\left|V, \Delta t_{S M F}+\Delta t_{M}\right\rangle .
$$

Next, after experiencing a rotation of $45^{\circ}$, the quantum state after the f-PBS is given by

$$
\begin{aligned}
|\psi\rangle_{S M F}= & \frac{1}{2} e^{i \phi_{M}}\left(|H, 0\rangle_{a}\left|V, \Delta t_{S M F}+\Delta t_{M}\right\rangle_{b}-|H, 0\rangle_{a}\left|H, \Delta t_{S M F}+\Delta t_{M}\right\rangle_{a}\right. \\
& \left.+|V, 0\rangle_{b}\left|V, \Delta t_{S M F}+\Delta t_{M}\right\rangle_{b}-|V, 0\rangle_{b}\left|H, \Delta t_{S M F}+\Delta t_{M}\right\rangle_{a}\right) .
\end{aligned}
$$


As opposed to first-order interference, the two-photon interference cannot be observed through the detection events of a single detector. Instead, the coincidence events registered by two detectors $\mathrm{D}_{a}$ and $\mathrm{D}_{b}$ located each at the outputs of the f-PBS are used to reveal the signature of this phenomenon. Therefore, we define the probability of observing a coincidence event between $\mathrm{D}_{a}$ and $\mathrm{D}_{b}$ as

$$
P_{S M F, \mathrm{D}_{a} \mathrm{D}_{b}}=\frac{1}{2}\left(1-e^{-\frac{4 \cdot \ln (2)\left(\Delta t_{S M F}+\Delta t_{M}\right)^{2}}{\tau_{c o h}^{2}}}\right),
$$

where, this time, $\tau_{c o h}$ denotes the coherence time of the photons which is defined by the transmission bandwidth of the employed band-pass filter.

The step-by-step elaboration of the second case of the two-photon interference experiment is actually the same as the first one. The only difference is that the PMF walk-off compensator is now introduced (see Fig.4.5 (c)) leading to a combined birefringence of the PMF itself and the natural one induced by the PPLN/W. From Eq.(4.6) to Eq.(4.8), the $\Delta t_{S M F}$ parameter can simply be represented by $\Delta t_{P M F}$ and it is stressed again that $\Delta t_{P M F}$ represents the combined birefringence across the experimental setup. For this reason, we can straightforwardly write the probability of having a coincidence event between $\mathrm{D}_{a}$ and $\mathrm{D}_{b}$ as follows:

$$
P_{P M F, \mathrm{D}_{a} \mathrm{D}_{b}}=\frac{1}{2}\left(1-e^{-\frac{4 \cdot \ln (2)\left(\Delta t_{P M F}+\Delta t_{M}\right)^{2}}{\tau_{c o h}^{2}}}\right) .
$$

From Eq.(4.9) and Eq.(4.10), we can clearly see that the corresponding coincidence probability goes down to zero when $\Delta t_{S M F}+\Delta t_{M}=0$ or $\Delta t_{P M F}+\Delta t_{M}=0$, for which this represents the optimal position of the HOM dip.

As can be seen in Fig.4.7, the obtained HOM dips correspond to different cases of twophoton interference measurement. The HOM dips are obtained through the registration of coincidence rate between detector $\mathrm{D}_{a}$ and $\mathrm{D}_{b}$ as a function of the delay scanner position, i.e. the position of the movable mirror. Without the PMF, i.e only with the SMF, the HOM dip is centered at a $\Delta t_{M}$ position of $-4.4 \mathrm{ps}$ which is in good agreement with the theoretical $\Delta t$ discussed earlier (refer to Eq.(4.1)). Meanwhile, with a $6 \mathrm{~m}$ long PMF, the HOM dip center is found at $\Delta t_{M}$ position of $3.38 \mathrm{ps}$. With this kind of information, we are able to compute the specific compensation factor concerning the use of PMF at 
$1540 \mathrm{~nm}$ which is $1.38 \mathrm{ps} / \mathrm{m}$. Accordingly, to erase the temporal walk-off effect induced by the natural PPLN/W brefringence, a $3.2 \mathrm{~m}$ long PMF should be added to the experimental setup. This is proven by a third HOM dip measurement where the the dip is now centered at $\Delta t_{M}=0.03 \mathrm{ps}$, indicating the temporal walk-off is nearly perfectly compensated.

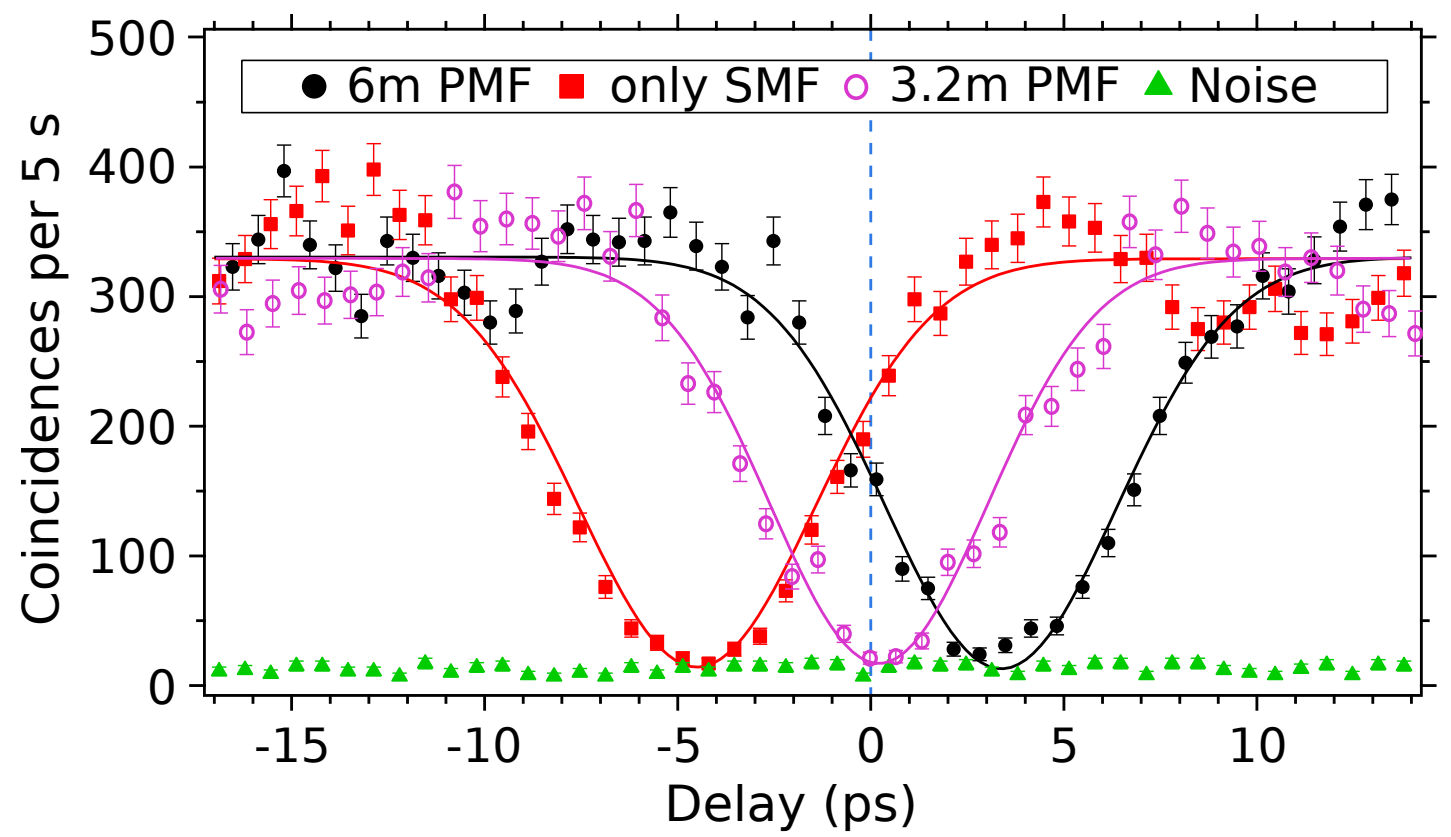

Figure 4.7: Hom dips with and without the PMF walk-off compensator. The associated raw visibilities for the three dips are $94 \pm 5 \%$. We note that, without the PMF compensator, the HOM dip is centered at $-4.4 \mathrm{ps}$ (red curve) which is in good agreement with the theoretical $\Delta t$ (refer to Eq.(4.1)). With a $6 \mathrm{~m}$ long PMF compensator, the HOM dip center is found at $3.38 \mathrm{ps}$ (black curve). Finally, with the estimated compensation length of $3.2 \mathrm{~m}$ of the PMF, the dip is centered at $\Delta t_{M}=0.03 \mathrm{ps}$ (magenta curve) indicating that the temporal walk-off is nearly perfectly compensated. The blue colored vertical dashed line symbolizes the zero delay position. As a side note, all of the above measurements are conducted with the help of two free-running indium-gallium-arsenide (InGaAs) avalanche photo-diodes (APD, IDQ 220 ) featuring each a quantum detection efficiency of $25 \%$ and a dark count probability of $10^{-6} / \mathrm{ns}$.

\subsubsection{Low-loss and deterministic photon pair separation}

In both two-photon interference experiments discussed in Sec.4.2.2, we saw that the photon pairs generated by the type-II PPLN/W are separated by a fiber polarizing beam splitter (f-PBS) acting like a 50/50 beam splitter. The latter is achieved by turning the photon polarization by $45^{\circ}$ in front of the f-PBS. In this case, there are only two out of four cases 
where a photon pair is actually separated. This leads to a separation probability of $\frac{1}{2}$ which represents $3 \mathrm{~dB}$ of loss affecting the measurement. This is what is known as probabilistic separation.

Nonetheless, we stated before that suitable filtering of the photon pairs can lead to two simultaneous advantages being:

- Ensuring high-quality entanglement by rejecting the unwanted photons in the mismatched regions of SPDC emission spectrua associated with different polarization modes $|H\rangle$ and $|V\rangle$;

- Achieving low-loss deterministic separation of photon pairs.

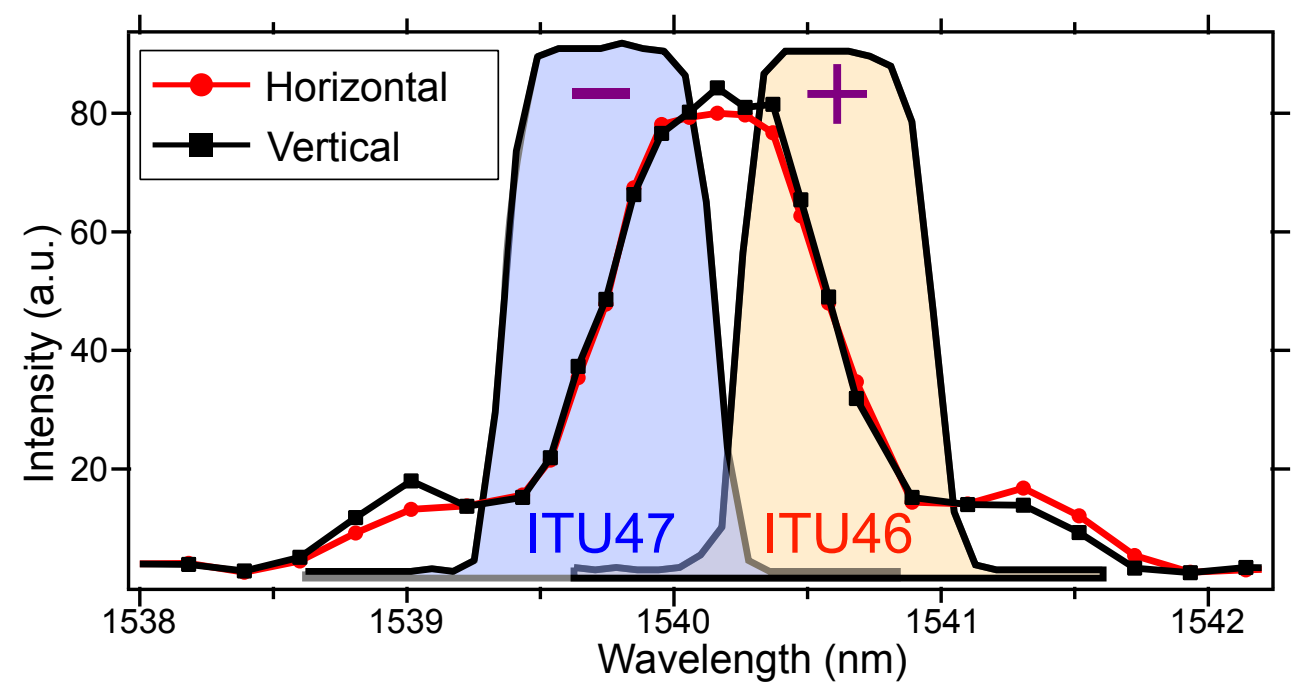

Figure 4.8: High (+) and low (-) regions of the SPDC emission spectrum. The filled curves represent the transmission spectra of the two employed DWDM filters used to deterministically separate the generated photon pairs and at the same time rejecting the unwanted photons in the mismatched regions.

The main idea toward such advantages is to divide the emission spectrum into two regions, namely the high $(+)$ and low (-) wavelength regions. As shown in Fig.4.8, the nonoverlapping outer side peaks are completely left out while most of the overlapping central peak is covered. Consequently, the unwanted photons on the side peaks are rejected while avoiding any major waste concerning the useful photons in the central peaks. This idea of deterministic separation of photon pairs is made possible thanks to the conservation of 
energy, stating that if one photon is found, for instance, in the + region, the other complementary, i.e. energy correlated, photon is found in - region. Furthermore, our choice of the SPDC phase matching condition permits us to have both + and - regions fell into standard, $100 \mathrm{GHz}$ ITU $^{2}$ channel 46 and 47, respectively. In conventional communication system, the ITU defines the frequency grid for dense wavelength division multiplexing (DWDM) technology which uses the full fiber bandwidth. Therefore, we can benefit from readily available, high-performance, low-loss, and cost-effective DWDM filters to actually separate the photon pairs such that two communicating parties Alice and Bob receive each a correlated photon. Let us assume that photons from the + region is destined for Alice. On the other hand, Bob receives the complementary photons belonging to the - region.

Interestingly, neither + nor - regions ever reveal the polarization state of the photons because the related emission spectra overlay perfectly (see Fig.4.8). Consequently, we obtain, in a coherent manner, $|H\rangle_{+}|V\rangle_{-}$or $|V\rangle_{+}|H\rangle_{-}$contributions, hence the generation of a maximally polarization entangled Bell state $\left|\Psi^{+}\right\rangle$,

$$
\left|\Psi^{+}\right\rangle=\frac{1}{\sqrt{2}}\left(|H\rangle_{+}|V\rangle_{-}+|V\rangle_{+}|H\rangle_{-}\right) .
$$

\subsubsection{Entanglement quality measurement}

Up to this point, a $3.2 \mathrm{~m}$ long polarization maintaining fiber (PMF) is introduced so as to erase the temporal walk-off induced by the PPLN/W birefringence. Furthermore, the deterministic separation of photon pairs is implemented thanks to a combination of two DWDM filters. Eventually, the maximally polarization entangled state $\left|\Psi^{+}\right\rangle=\frac{1}{\sqrt{2}}\left(|H\rangle_{+}|V\rangle_{-}+|V\rangle_{+}|H\rangle_{-}\right)$is shared between Alice and Bob. To actually measure or quantify the level of entanglement contained in such a state, a simple experimental setup of entanglement analyzer is implemented (see Fig.4.9). To this end, Alice and Bob both possess identical arrangement of entanglement analyzer involving a fiber polarization controller (PC), a half-wave plate (HWP), a polarization beam splitter (PBS) and an avalanche photo-diode (APD). Both employed APDs are again free-running InGaAs detectors (IDQ 220 ) featuring each $20 \%$ of quantum detection efficiency and $\sim 10^{-6} /$ ns dark count prob-

\footnotetext{
${ }^{2}$ ITU stands for International Telecommunication Union, an international organization headquartered in Geneva.
} 


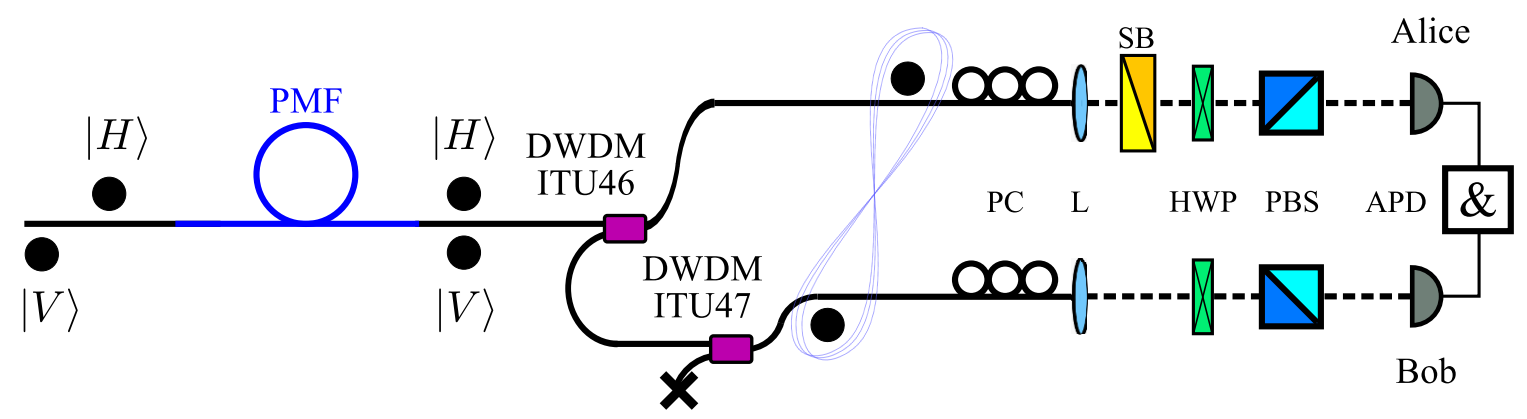

Figure 4.9: Experimental apparatus for quantifying the level of entanglement contained in the generated maximally polarization entangled state of $\left|\Psi^{+}\right\rangle=\frac{1}{\sqrt{2}}\left(|H\rangle_{+}|V\rangle_{-}+\right.$ $\left.|V\rangle_{+}|H\rangle_{-}\right)$. Alice and Bob implement identical arrangement of entanglement analyzer comprising a fiber polarization controller (PC), a half-wave plate (HWP), a polarization beam splitter (PBS) and an avalanche photo-diode (APD). Particularly on Alice's side, a Soleil-Babinet (SB) compensator is placed along other entanglement analyzer elements so as to compensate any potential biefringence induced by the employed single mode fibers (SMF) after the photon pair separation.

ability. A coincidence counter (\&) is also employed to acquire the coincidence detection events.

\subsubsection{Phase measurement}

With an exception to Alice's analyzer, she is equipped with one more optical component, being a Soleil-Babinet (SB) compensator so as to compensate any potential biefringence induced by the employed single mode fibers (SMF) after the photon pair separation. Due to such birefringence, a phase relation $\phi$ can be introduced between the contributions of the entangled state $\left|\Psi^{+}\right\rangle$. This way, Eq.(4.11) can be rewritten as

$$
|\Psi(\phi)\rangle=\frac{1}{\sqrt{2}}\left(|H\rangle_{+}|V\rangle_{-}+e^{i \phi}|V\rangle_{+}|H\rangle_{-}\right)
$$

As of our aim to generate the maximally entangled Bell state $\left|\Psi^{+}\right\rangle$with no phase relation between its two contributions, mainly for ease of analysis purposes, the phase $\phi$ needs to be canceled. Alternatively, the phase $\phi$ can also be set to $\pi$ if one decides to produce the other Bell state $\left|\Psi^{-}\right\rangle$, also maximally entangled. For this reason, the two HWP on both 
sides of the experiment are rotated to $22.5^{\circ}$ such that we transform Eq.(4.12) into

$$
\begin{aligned}
|\Psi(\phi)\rangle_{22.5^{\circ}} & =\frac{1}{2 \sqrt{2}}\left(\left(|H\rangle_{+}+|V\rangle_{+}\right)\left(|V\rangle_{-}-|V\rangle_{-}\right)+e^{i \phi}\left(|V\rangle_{+}-|H\rangle_{+}\right)\left(|H\rangle_{-}+|V\rangle_{-}\right)\right) \\
& =\frac{1+e^{i \phi}}{2} \cdot \underbrace{\frac{\left(|V\rangle_{+}|V\rangle_{-}-|H\rangle_{+}|H\rangle_{-}\right)}{\sqrt{2}}}_{\text {coincidence }}+\frac{1-e^{i \phi}}{2} \cdot \underbrace{\frac{\left(|V\rangle_{+}|V\rangle_{-}-|H\rangle_{+}|H\rangle_{-}\right)}{\sqrt{2}}}_{\text {anti-coincidence }} .
\end{aligned}
$$

As we are dealing with coincidence events between the two employed single photon detectors, only photons that are simultaneously transmitted (or reflected) by both PBS are concerned. In this regard, only $|V\rangle_{+}|V\rangle_{-}$and $|H\rangle_{+}|H\rangle_{-}$contributions out of Eq.(4.13) can give rise to coincidence events. Correspondingly, the other two contributions $|H\rangle_{+}|V\rangle_{-}$and $|V\rangle_{+}|H\rangle_{-}$yield anti-coincidence events. Thus, we can define two probabilities concerning each observation as follows:

$$
\begin{aligned}
P_{\text {coinc }} & =\cos ^{2}\left(\frac{\phi}{2}\right) . \\
P_{\text {anti-coinc }} & =\sin ^{2}\left(\frac{\phi}{2}\right) .
\end{aligned}
$$

According to Eqs.(4.15) and (4.15), the phase $\phi$ indeed represents a strong experimental parameter affecting the coincidence rate of the measurement. For instance, a straightforward calculation of imposing $\phi=0$ in Eq.(4.15) yields a unit probability of observing coincidence events. This can be observed experimentally through the use of the Soleil-Babinet compensator where the coincidence rate oscillate sinusoidally with the artificial phase introduced by this device. The observed sinusoidal oscillation of the coincident rate is shown in Fig.4.10.

As expected, the observed coincidence rate attains its maximum at $\phi=0$. It is also interesting to note that at $\phi=\pi$, the rate drops to its minimum. As a matter of fact, these two conditions on the $\phi$ parameter corresponds to the generation of two maximally entangled Bell states, being $\left|\Psi^{+}\right\rangle$for $\phi=0$, and $\left|\Psi^{-}\right\rangle$for $\phi=\pi$. 


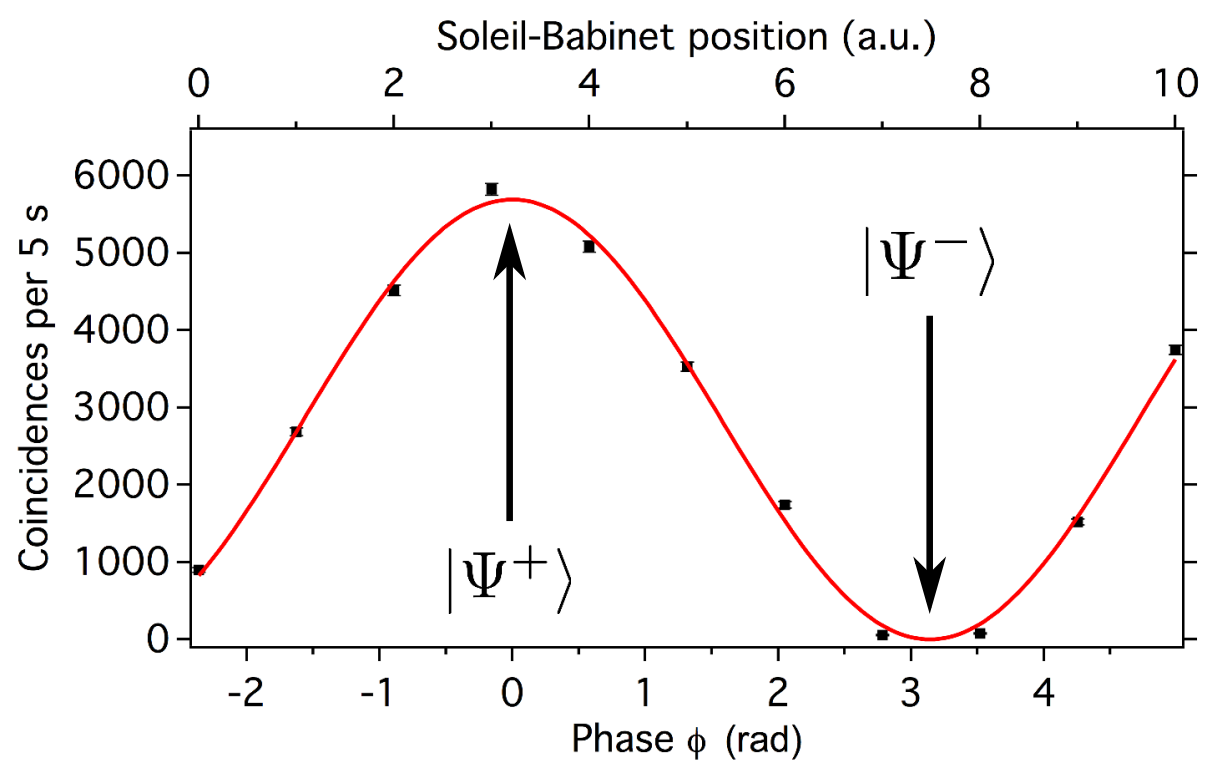

Figure 4.10: The observed sinusoidal oscillation of the coincident rate. The two experimental points correspond to the maximum and the minimum coincidence rates symbolize the generation of two maximally entangled Bell states of $\left|\Psi^{+}\right\rangle$for $\phi=0$, and $\left|\Psi^{-}\right\rangle$for $\phi=\pi$.

\subsubsection{Entanglement analysis}

From the previous measurement, we deliberately set the Soleil-Babinet compensator such that the phase relation $\phi$ is canceled, thus mandating the generation of the maximally polarization entangled Bell state $\left|\Psi^{+}\right\rangle=\frac{1}{\sqrt{2}}\left(|H\rangle_{+}|V\rangle_{-}+|V\rangle_{+}|H\rangle_{-}\right)$. At this point, the registered coincidence rate attains its maximum. Now, we suppose that Alice's and Bob's respective half-wave plate (HWP) are turned by the same angle. According to quantum theory, we should expect the same coincidence rate as the correlation induced by the state $\left|\Psi^{+}\right\rangle$is indeed invariant by rotation of the analyzer's orientation. Otherwise, we are dealing with a classical mixture of states.

In order to prove what is predicted by quantum theory and at the same time violating the Bell-CHSH inequality [Clauser et al. 1969], we first define the following standard orientation for Alice's HWP:

- $0^{\circ} \Rightarrow|H\rangle$

- $22.5^{\circ} \Rightarrow|D\rangle$

- $45^{\circ} \Rightarrow|V\rangle$; 
$-22.5^{\circ} \Rightarrow|A\rangle$.

For each of the above, the detection rates between the two employed detectors are recorded as a function of the continuous change of Bob's HWP angle. With the data, we are able to plot the so-called correlation curves which represents the simplest proof of quantum entanglement. As can be seen in Fig.4.11, the coincidence rates oscillate sinusoidally as a function of the angle of Bob's HWP on all four occasions. Thus, we can confidently say that the observed correlations are indeed induced by quantum entanglement as a measurement on one side is correlated to the measurement on the other side. Therefore, this further demonstrates the inseparability in term of polarization states that belongs to an entangled photon pair. Such strong correlation in polarization can be quantified through the visibility of the oscillations which can be defined as

$$
V=\frac{C_{\max }-C_{\min }}{C_{\max }+C_{\min }}
$$

where $C_{\max }\left(C_{\min }\right)$ represents the maximum (minimum) coincidence rate. In order to violate Bell's inequality, the average visibility of the oscillations should exceed $71 \%$. In this regard, the higher the visibility, the greater will be the entanglement quality. As in our case, the raw visibility is computed to be $V_{\text {raw }}=97.3 \pm 0.6 \%$. When subtracting the accidental coincidence events due to the dark counts in the detectors, we obtain the net visibility, $V_{\text {net }}=99.5 \pm 0.8 \%$.

Another way of proving entanglement can be done by computing the so-called Bell parameter $S$ from the measurement outcomes which is defined by (see also 1.2.3.3)

$$
S=E(\vec{a}, \vec{b})+E\left(\vec{a}^{\prime}, \vec{b}\right)+E\left(\vec{a}^{\prime}, \vec{b}^{\prime}\right)-E\left(\vec{a}, \vec{b}^{\prime}\right)
$$

where

$$
E(\vec{a}, \vec{b})=\frac{C(\vec{a}, \vec{b})-C\left(\vec{a}, \overrightarrow{b_{\perp}}\right)-C\left(\overrightarrow{a_{\perp}}, \vec{b}\right)+C\left(\overrightarrow{a_{\perp}}, \overrightarrow{b_{\perp}}\right)}{C(\vec{a}, \vec{b})+C\left(\vec{a}, \overrightarrow{b_{\perp}}\right)+C\left(\overrightarrow{a_{\perp}}, \vec{b}\right)+C\left(\overrightarrow{a_{\perp}}, \overrightarrow{b_{\perp}}\right)}
$$

Here, $C(\vec{a}, \vec{b})$ denotes the amount of obtained coincidences for a combination of HWP orientation $\vec{a}$ and $\vec{b}\left(\overrightarrow{a_{\perp}}\right.$ and $\overrightarrow{b_{\perp}}$ are perpendicular to $\vec{a}$ and $\vec{b}$, respectively). By extracting the experimental points from Fig.4.11, we are able to compute the Bell parameters 


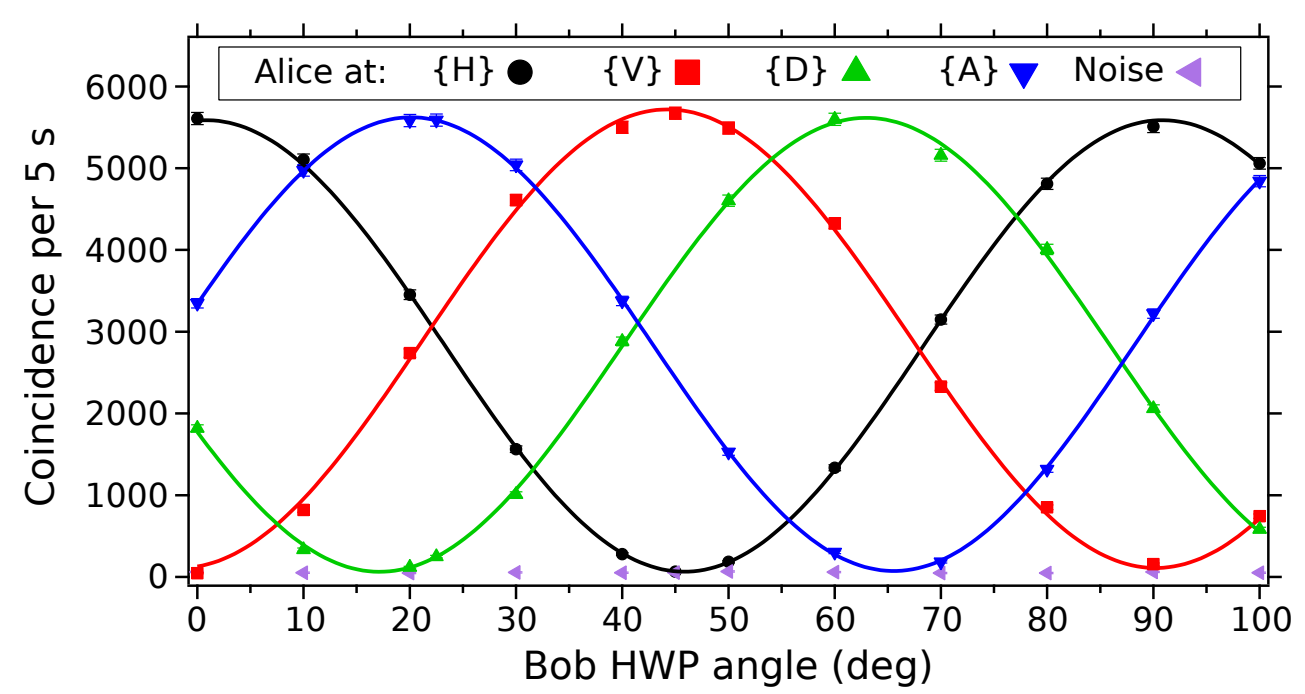

Figure 4.11: The obtained correlation curves. The coincidence rates oscillate sinusoidally as a function of the angle of Bob's HWP for all settings on Alice's HWP. A direct observation of these curves represents the simplest proof of quantum entanglement.

$S_{\text {raw }}=2.806 \pm 0.005$ and $S_{\text {net }}=2.824 \pm 0.007$ which violates the classical limit $S=2$ by more than 100 standard deviations for each value.

\subsubsection{Entangled state symmetry manipulation}

The two-photon interference experiments performed in Sec.4.2.2 involves wavelength degenerate photon pairs. In those cases, the type-II SPDC emission spectrum is filtered by a $0.8 \mathrm{~nm}$ band-pass optical filer centered at the degenerate wavelength of the emission spectrum $(1540 \mathrm{~nm})$. Even though the two photons are cross-polarized, i.e they are distinguishable in term of polarization, the HOM interference effect can still be observed in such experiment.

To explain this, assume that a pair of independent single photons is made available for the interference at a polarizing beam splitter (PBS) and it is defined by the state $|\psi\rangle=|H\rangle|V\rangle$. Then, their polarization states are turned by $45^{\circ}$, therefore the state $|\psi\rangle$ reads

$$
|\psi\rangle=\frac{1}{2}(|H\rangle|V\rangle-|H\rangle|H\rangle+|V\rangle|V\rangle-|V\rangle|H\rangle)
$$

If these two photons are indistinguishable in any other degree of freedom, namely the spatial, spectral and temporal modes except for their polarization upon impinging the PBS, 
the $|H\rangle|V\rangle$ and $|V\rangle|H\rangle$ contributions interfere destructively. Therefore, only $|H\rangle|H\rangle$ and $|V\rangle|V\rangle$ contributions are left and as a consequence, both photons will always exit the PBS through the same output. This is how the indistinguishability between two cross-polarized photons can be quantified by a HOM-type experiment.

Surely, the above arguments can be used to explain the HOM dips observed in Sec.4.2.2. However, could we base on other fundamental reasons to explain the observed HOM dips? Nevertheless, the above explanation cannot be used to describe the dip observed in all HOM-related experiments, at least in our next proposed experiment. To prove this, we will later demonstrate a new set of two-photon interference experiments involving cases where two separated photons arrive at a fiber PBS in an untimely manner. Concurrently, we also benefit from the manipulable, constant phase relation $\phi$ between the two contributions of the general entangled state $|\Psi(\phi)\rangle=\frac{1}{\sqrt{2}}\left(|H\rangle_{+}|V\rangle_{-}+e^{i \phi}|V\rangle_{+}|H\rangle_{-}\right)$in order to deliberately produce the maximally polarization entangled Bell states being either $\left|\Psi^{+}\right\rangle$or $\left|\Psi^{-}\right\rangle$.

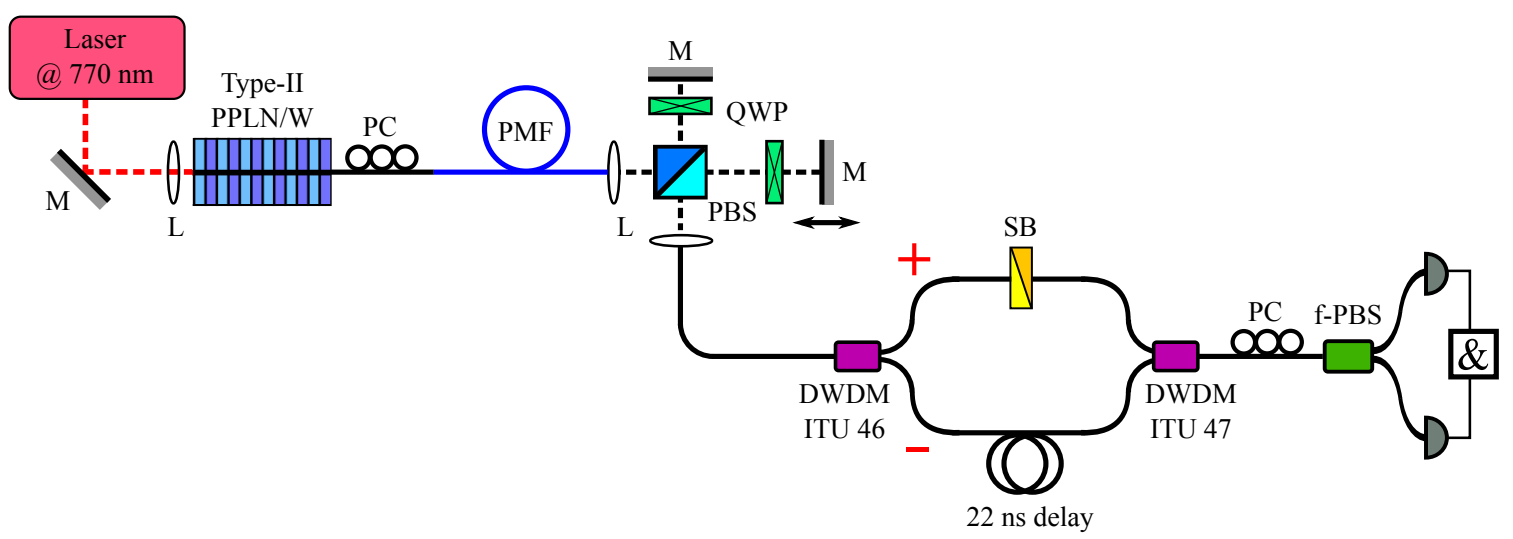

Figure 4.12: Experimental setup for manipulating the quantum state symmetry. Crosspolarized photon pairs are made available after the delay scanner apparatus. The pairs are then deterministically separated by the action of the first DWDM that belongs to an interferometer-like configuration, such it splits paired photons that can be associated with two different wavelength region of + and -. + label photons travel in the short arm of the interferometer and experience a certain phase shift $\phi$ induced by the Soleil-Babinet compensator. Meanwhile, - photons that propagate in the long arm experience a delay of $22 \mathrm{~ns}$. The paired photons are then recombined into a single spatial mode by another DWDM. Afterward, the polarization state of the photon pairs are rotated by $45^{\circ}$ using a PC and they are subsequently subjected to an f-PBS having two detectors at its outputs.

We consider the experimental setup depicted in Fig.4.12. Basically, one should be already familiar with most parts of the experiment. As usual, cross-polarized photon pairs are 
made available after the delay scanner apparatus. The photon pairs then first encounter a DWDM (ITU 46) which belongs to a Mach-Zehnder interferometer-type arrangement. Things are getting interesting when the photon pairs are deterministically separated by the action of the DWDM, such that paired photons are split and can be associated with two different wavelength regions + and -. Just after the separation, the quantum state reads

$$
\left|\Psi^{+}\right\rangle=\frac{1}{\sqrt{2}}\left(|H\rangle_{+}|V\rangle_{-}+|V\rangle_{+}|H\rangle_{-}\right) .
$$

The Soleil-Babinet (SB) is positioned in the + arm so as to introduce a phase shift between the $|H\rangle_{+}$and $|V\rangle_{+}$contributions. Meanwhile, in the opposite arm, the contributions $|H\rangle_{\text {- }}$ and $|V\rangle_{\text {- }}$ experience a delay of $22 \mathrm{~ns}$ which is equivalent to a $5 \mathrm{~m}$ long optical fiber. Eventually, another DWDM (ITU 47) is employed to recombine the photon pairs back to a single spatial mode. And now comes the big question on how we describe the state after such recombination of photon pairs.

For discussion's sake, let us assume hypothetically that the SB introduces a zero phase $(\phi=0)$ and that the delay between the two arms of the interferometer is also zero $(d=0)$, such that both photons arrive exactly at the same time at the f-PBS. One would consider to describe, for instance, the state $|\psi\rangle_{\text {recombine }}=|H\rangle_{+}|V\rangle_{-}$to the photon pairs exiting the interferometer. The polarization of the pairs are turned by $45^{\circ}$ by a $\mathrm{PC}$ and the pairs are then sent to an f-PBS. The quantum state after the f-PBS reads

$$
|\psi\rangle_{\text {recombine }}=\frac{1}{2}\left(|H\rangle_{+}|V\rangle_{-}-|H\rangle_{+}|H\rangle_{-}+|V\rangle_{+}|V\rangle_{-}|V\rangle_{+}|H\rangle_{-}\right) .
$$

Obviously, in this hypothetical situation, two-photon interference concerning crosspolarized photons is absent due to the additional wavelength labeling on photons that reside in the same pair. This is very contradictory to what we actually observed in the actual experiment, not to mention the fact that the separated photons never arrived at the f-PBS at the same time.

From now on, let us take the high road by considering the real implementation of the experiment. Due to the phase $\phi$ introduced by the SB and the delay $d$ between + and - 
contributions, we obtain after recombination

$$
|\psi\rangle_{\text {recombine }}=\frac{1}{\sqrt{2}}\left(|V, 0\rangle_{+}|H, d\rangle_{-}+e^{i \phi}|H, 0\rangle_{+}|V, d\rangle_{-}\right) .
$$

After a rotation of $45^{\circ}$ by the $\mathrm{PC}$, we have

$$
|\psi\rangle_{P C}=\frac{1+e^{i \phi}}{2} \cdot \underbrace{\frac{\left(|V, 0\rangle_{+}|V, d\rangle_{-}-|H, 0\rangle_{+}|H, d\rangle_{-}\right)}{\sqrt{2}}}_{\text {anti-coincidence }}+\frac{1-e^{i \phi}}{2} \cdot \underbrace{\frac{\left(|H, 0\rangle_{+}|V, d\rangle_{-}-|V, 0\rangle_{+}|H, d\rangle_{-}\right)}{\sqrt{2}}}_{\text {coincidence }}
$$

According to Eq.(4.23), only photons pairs that are separated by the f-PBS can give rise to the coincidences. Meanwhile, photons that exit through the same output of the f-PBS correspond to the case of anti-coincidences. The probabilities of observing such cases are given by

$$
\begin{aligned}
P_{\text {coinc }} & =\sin ^{2}\left(\frac{\phi}{2}\right) . \\
P_{\text {anti-coinc }} & =\cos ^{2}\left(\frac{\phi}{2}\right) .
\end{aligned}
$$

The contributions related to the coincidences (anti-coincidences) always show an even (odd) spatial distribution due to their bosonic (fermionic) nature. This corresponds to the maximally entangled Bell state of $\left|\Psi^{+}\right\rangle\left(\left|\Psi^{-}\right\rangle\right)$, associated with an even (odd) wave-function parity. The "switching" between the symmetry of the entangled state can be conveniently achieved by fixing the phase $\phi$, from 0 to $\pi$, corresponding to $\left|\Psi^{+}\right\rangle$and $\left|\Psi^{-}\right\rangle$, respectively.

A set of two-photon interference experiment for $\phi=0$ and $\phi=\pi$ is conducted where the outcomes are represented in Fig.4.13. As for $\phi=0$, the state $\left|\Psi^{+}\right\rangle$is generated, thus the photon coalescence in the form of a HOM dip is observed. When the the phase $\phi$ is set to $\pi$, we deliberately turn the state $\left|\Psi^{+}\right\rangle$into $\left|\Psi^{-}\right\rangle$. As expected, a HOM peak is obtained as the manifestation of the anti-coalescence behavior. Remarkably, both HOM dip and peak are observed despite the "interfering" photons are not at the same wavelength and are timedelayed by $22 \mathrm{~ns}$. As a matter of fact, after the deterministic separation by the DWDM, they never meet each other as the $22 \mathrm{~ns}$ delay is much greater than their coherence $(\sim 5 \mathrm{ps})$. 
Therefore, the observed interference is not due to conventional two-photon interference.

Let us rewind a little bit. We assumed $\phi$ is set to zero previously and after the recombination of paired photons by the DWDM, we have a quantum state $\left|\Psi^{+}\right\rangle=$ $\frac{1}{\sqrt{2}}\left(|V, 0\rangle_{+}|H, d\rangle_{-}+|H, 0\rangle_{+}|V, d\rangle_{-}\right)$. Is there any way to tell which contribution is responsible for a detected coincidence between the two detectors after the f-PBS? The answer is no even though we are aware of the fact that both photons have different wavelengths ( + or - ) and one of them is greatly delayed in time compared to the other. In fact, the two contributions are indeed indistinguishable and they simply interfere.

This indicates that this quantum effect, i.e. the interference observed in this particular experiment resulting either in HOM dip or HOM peak, does not simply originate from a conventional two-photon interference, but rather from an entangled state post-selected by the measurement apparatus. Ultimately, the photon pairs produced by the type-II PPLN/W are directly and naturally available in an entangled state after the PM fiber.

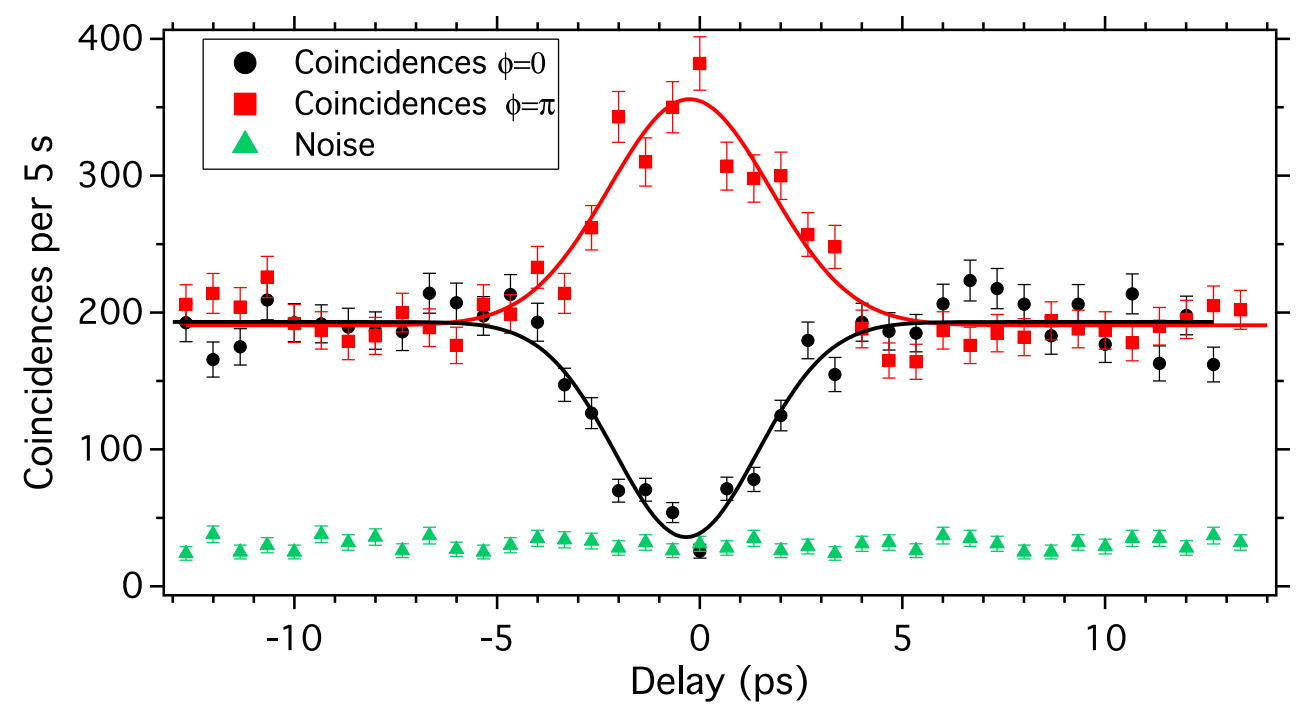

Figure 4.13: HOM dip and peak obtained for two different settings of the Soleil-Babinet compensator. For $\phi=0$, the state $\left|\Psi^{+}\right\rangle$is generated, thus photon coalescence in the form of a HOM dip is observed. Changing the phase to $\pi$ turns the state to $\left|\Psi^{-}\right\rangle$for which a HOM peak is obtained as the manifestation of the anti-coalescence behavior.

\subsubsection{Source's performances}

Once the correlated photons are observed in term of coincidence events, two important figures of merit concerning the performance of our type-II photon pair generator can be 
conveniently measured experimentally, namely:

- The brightness $B$ of the SPDC source [Tanzilli et al. 2002];

- The total internal SPDC efficiency $\eta_{S P D C}$ [Tanzilli et al. 2001, Tanzilli et al. 2002].

The normalized brightness $B$ of an SPDC source simply means the expected amount of photon pairs $\left(N_{\text {pair }}\right)$ being generated per $\mathrm{mW}$ of pump power, per $1 \mathrm{~s}$ of integration time, and per $\mathrm{GHz}$ of spectral bandwidth, which is described by:

$$
B=\frac{N_{\text {pair }}}{\mathrm{mW} \cdot \mathrm{s} \cdot \mathrm{GHz}}
$$

Our method to measure the brightness of the SPDC source is inspired by previous work reported in [Tanzilli et al. 2002]. First and foremost, in the pursuance of estimating the numerator $N_{\text {pairs }}$, of Eq.(4.26), the following experimental parameters in the most original configuration shown in Fig.4.1 are measured:

- $S_{1}$ : the single count rate recorded by Bob's detector per second;

- $S_{2}$ : the single count rate recorded by Alice's detector per second;

- $C$ : the coincidence rate between the two detectors per second.

In the following, the role of the above parameters $S_{1}, S_{2}$ and $C$ in evaluating $N_{\text {pairs }}$ is progressively described. To begin with, $S_{1}$ can be theoretically defined by

$$
S_{1}=\frac{1}{2} \mu_{1} \eta_{1} N_{\text {pair }} \rightarrow \frac{1}{2} \mu_{1} \eta_{1}=\frac{S_{1}}{N_{\text {pair }}}
$$

Here, $\mu_{1}$ represents the overall transmission from the SPDC waveguide until Bob's detector whose quantum detection efficiency is denoted by $\eta_{1}$. Note that the factor $\frac{1}{2}$ comes from the PBS found inside Alice's and Bob's respective entanglement analyzers. Due to the PBS, half of the photons are lost. Similarly, $S_{2}$ can be described as:

$$
S_{2}=\frac{1}{2} \mu_{2} \eta_{2} N_{\text {pair }} \rightarrow \frac{1}{2} \mu_{2} \eta_{2}=\frac{S_{2}}{N_{\text {pair }}} .
$$


Meanwhile, the coincidences $C$ between Alice's and Bob's respective detectors:

$$
C=\frac{1}{2} \mu_{1} \eta_{1} \mu_{2} \eta_{2} N_{\text {pair }}
$$

Finally, by plugging in the parameters $\frac{1}{2} \mu_{1} \eta_{1}$ and $\frac{1}{2} \mu_{2} \eta_{2}$ in Eq.(4.29) by their previously derived values from Eq.(4.27) and (4.28), respectively, we obtain

$$
N_{\text {pairs }}=\frac{2 S_{1} \cdot S_{2}}{C}
$$

In our case, with a pump power of $1 \mathrm{~mW}$ in front of the SPDC waveguide, we obtained $S_{1}=28000 \mathrm{~s}^{-1}$ in Bob's detector, $S_{2}=25000 \mathrm{~s}^{-1}$ in Alice's detector and $C=1100 \mathrm{~s}^{-1}$ prompting a $N_{\text {pair }}$ of $1.25 \cdot 10^{6} \mathrm{~s}^{-1}$. Thus, this leads to a brightness of

$$
B=\frac{1.27 \cdot 10^{4}}{\mathrm{~mW} \cdot \mathrm{s} \cdot \mathrm{GHz}}
$$

for our type-II photon pair generator.

Meanwhile, by definition, the total internal SPDC efficiency, $\eta_{S P D C}$, represents the probability of successfully generating a photon pair from a single pump photon, within the full emission bandwidth of the PPLN waveguide, formulated by the following equation

$$
\eta_{S P D C}=\frac{N_{\text {pair }}}{N_{\text {pump }}}
$$

Here, $N_{\text {pump }}$ represents the number of pump photons injected into the SPDC waveguide and can be defined as

$$
N_{\text {pump }}=\frac{P_{\text {pump }} \cdot \lambda_{\text {pump }}}{h \cdot c}
$$

where $P_{\text {pump }}$ is the coupled pump power measured in $\mathrm{W}, \lambda_{\text {pump }}$ defines the pump wavelength (in our case $770 \mathrm{~nm}), h$ represents Planck's constant $\left(h=6.62607 \cdot 10^{-34} \mathrm{~J} \ldots\right)$ and $c$ is the speed of light in vacuum $\left(c=299792458 \mathrm{~m} \cdot \mathrm{s}^{-1}\right)$.

Being closely related to the brightness $B$ of the SPDC source through the term $N_{\text {pair }}$, $\eta_{S P D C}$ can be further rewritten as

$$
\eta_{S P D C}=\left(B \cdot P_{\text {pump }} \cdot \Delta \nu \cdot 10^{3}\right) \times\left(\frac{h \cdot c}{P_{\text {pump }} \cdot \lambda_{\text {pump }}}\right) .
$$


Table 4.1: Experimental results for different state-of-the-art type-II polarization entanglement, waveguide or bulk, source. $\eta_{S P D C}$ : SPDC efficiency, $V_{\text {net }}$ : calculated net entanglement visibility by subtracting the accidental events, DSF: dispersion shifted fiber, PPKTP: periodically poled potassium titanyl phosphate.

\begin{tabular}{lllll}
\hline Reference & Configuration & $\eta_{S P D C}$ & $V_{\text {net }}$ & Coincidence rate \\
\hline [Lee et al. 2006] & DSF & $3.2 \cdot 10^{-32}$ & $98.3 \%$ & $80 \mathrm{~s}^{-1}$ \\
[Suhara et al. 2007$]$ & type-II PPLN/W & $5.3 \cdot 10^{-10}$ & $90 \%$ & $\mathrm{NA}$ \\
[Piro et al. 2009] & type-II PPKTP & $2.8 \cdot 10^{-10}$ & $98 \pm 1 \%$ & $5 \mathrm{~s}^{-1}$ \\
[Medic et al. 2010$]$ & DSF & $\mathrm{NA}$ & $99.4 \pm 1.2 \%$ & $5 \mathrm{~s}^{-1}$ \\
[Martin et al. 2010$]$ & type-II PPLN/W & $1.1 \cdot 10^{-9}$ & $99 \pm 2 \%$ & $800 \mathrm{~s}^{-1}$ \\
This thesis [Kaiser et al. 2012b] & type-II PPLN/W & $3.5 \cdot 10^{-10}$ & $99.5 \pm 0.8 \%$ & $1100 \mathrm{~s}^{-1}$ \\
\hline
\end{tabular}

It is interesting to note that in order to evaluate $\eta_{S P D C}$, we only consider the natural emission bandwidth of the employed SPDC waveguide. This way, the calculated $\eta_{S P D C}$ is not just limited to specific cases where spectral filtering of the generated photons is implemented, but also satisfies the scenario of applications where no spectral filtering is required. Thus, within Eq.(4.34), $\Delta \nu$ represents the natural emission bandwidth of the $\mathrm{SPDC}$ waveguide in $\mathrm{GHz}$ (in our case, the emission bandwidth of $0.85 \mathrm{~nm}$ corresponds to $107 \mathrm{GHz})$. Apart from that, the factor $10^{3}$ is introduced in such equation due to the fact that the previous brightness $B$ is measured per $\mathrm{mW}$ of pump power. By substituting all the parameters in Eq.(4.34) with their respective values, we show that

$$
\eta_{S P D C}=3.5 \cdot 10^{-10} \frac{\text { pairs }}{\text { pump photon }} .
$$

In Tab.A.1, our measured SPDC efficiency value is compared to other realizations of type-II polarization entanglement sources.

In addition, the propagation losses of about $3 \mathrm{~dB}$ and $3.5 \mathrm{~dB}$ are measured for both Alice's and Bob's respective single photons, from the output of the generator to the measurement apparatus. This is an exceptionally good loss figure, and mainly ascribed to the advantageous use of off-the-shelf standard telecom components, notably the DWDMs for deterministic separation of photon pair. Note that the main loss of around $2 \mathrm{~dB}$ comes from the butt-coupling between the PPLN/W and the collection optical fiber. 


\subsection{Conclusion and perspectives}

In conclusion, we have demonstrated a compact, high-quality polarization entanglement source based on a non-linear type-II SPDC interaction in a PPLN/W. The reliability of the source is guaranteed through the use of high performance standard telecom components notably, the standard polarization maintaining fiber for waveguide birefringence compensation and a set of DWDMs for deterministic pair separation into two standard telecommunication channels ITU 46 and 47 . The observed excellent quality entanglement obtained through the Bell-CHSH inequality test, combined with the simplicity of the setup, make this approach a good candidate for future quantum networking solutions based on photonic entanglement.

In the meantime, the compactness of the source can be further improved if the output of the $770 \mathrm{~nm}$ main pump laser is pigtailed to an optical fiber. Moreover, the same pigtailing concept can also be applied on both input and output facets of the PPLN/W. Overall, this would provide more stabilization to the system and at the same time considerably improves collection efficiency of the generated photon pairs out of the waveguide. Alternatively to PPLN/W pigtailing, one can implement a segmented tapered waveguide structure for enabling a better channel waveguide to fiber mode overlap [Castaldini et al. 2009]. Moreover, the free-space based entanglement analyzer can be replaced with a fully-fibered one, comprising a piezo-driven fiber polarization controller and a fiber polarizing beam splitter. Note that the free-space configuration of the entanglement analyzer typically reduces the single photon transmission by $10 \%$. 



\title{
Versatile polarization entanglement
}

\author{
source based on a type-0 PPLN/W
}

\section{Contents}

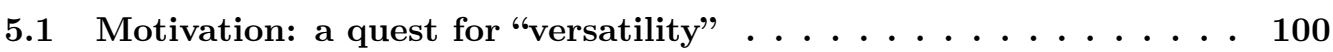

5.2 Versatile polarization entanglement source: the experiment . . . 100

5.2 .1 Photon pair generation via type-0 SPDC . . . . . . . . . . . . . . 102

5.2.2 Adaptable photon bandwidth via suitable spectral filtering . . . . . . 102

5.2 .3 Polarization entanglement preparation stage . . . . . . . . . . . . 106

5.2 .4 Phase stabilization . . . . . . . . . . . . . . . . . 111

5.2.5 Filter-associated coincidence histogram . . . . . . . . . . . . . 114

5.2 .6 Entanglement analysis . . . . . . . . . . . . . . . . . . 116

5.2 .7 Source's performances . . . . . . . . . . . . . . . . . . 118

5.3 Conclusion and perspectives $\ldots \ldots \ldots \ldots \ldots \ldots$

Some results presented in this chapter were published in:

- F. Kaiser, A. Issautier, L. A. Ngah et al., A versatile source of polarisation entangled photons for quantum network applications, Laser Physics Letters, Volume 10, pages 045202, February 2013.

- F. Kaiser, L. A. Ngah et al., Polarization entangled photon-pair source based on quantum nonlinear photonics and interferometry, Optics Communications, Volume 327, Special Issue on Nonlinear Quantum Photonics, pages 7-16, September 2014. 


\subsection{Motivation: a quest for "versatility"}

In this chapter, we are interested in demonstrating the generation of polarization entanglement by taking advantage of type-0 spontaneous parametric down conversion (SPDC) process in periodically poled lithium niobate waveguide (PPLN/W). Contrarily to type-II, type-0 is known to yield around two orders of magnitude higher interaction efficiency due to the exploitation of the $d_{33}$ coefficient which is the most efficient non-linear coefficient in LiNbO3. Consequently, a reasonable overall experimental rate can still be maintained even though the generated photons are subjected to suitable narrowband filtering. In this regard, the photons produced by our source can be engineered, in term of their spectral bandwidth, to be adapted to broad variety of quantum applications, namely QKD in standard 200, 100, or $50 \mathrm{GHz}$ ITU channels, as well as cold-atom based quantum memory applications exhibiting absorption bandwidth up to a few tens of MHz. Furthermore, our type-0 source emits photon pairs at a wavelength that belongs to the C-band $(1530-1565 \mathrm{~nm})$ telecom specification, thus allowing long distance propagation in standard optical fibers. Concerning the worries about the polarization observable for long distance quantum communications, active compensation of fiber birefringence fluctuations can be now adopted to ensure an overall polarization stability [Chen et al. 2007, Xavier et al. 2009, Chen et al. 2009]. Last but not least, the information inferred by the polarization state of single photons can be conveniently analyzed in an interferometer-free environment, using only simple linear optical components.

\subsection{Versatile polarization entanglement source: the experi- ment}

The different building blocks of our versatile photonic polarization entanglement source are depicted in Fig.5.1. Throughout this section, we will comprehensively describe the source by respecting the chronology order, starting from the generation of photon pairs via type- 0 SPDC in a PPLN/W, down to the demonstration of high-quality polarization entanglement via the violation of the Bell-CHSH inequality. 


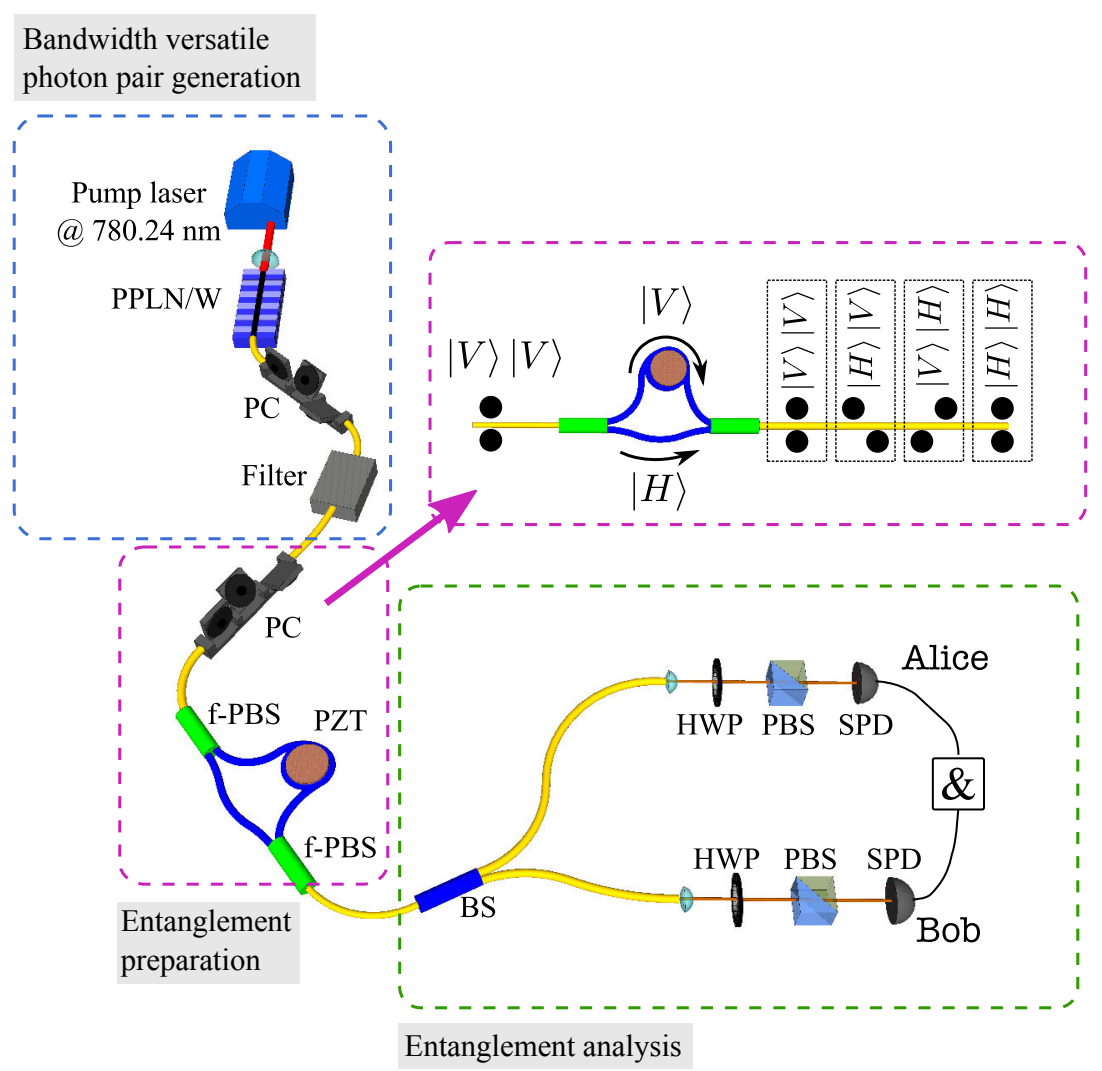

Figure 5.1: The effective building blocks of our "versatile" polarization entanglement source based on non-linear type-0 SPDC process. A diode laser is employed to provide the required SPDC pump field at $780.24 \mathrm{~nm}$. It allows generating co-polarized photon pairs in the type- 0 PPLN/W. The photon pairs are then collected by a single mode telecom fiber and directed toward a suitable filter whose transmission bandwidth corresponds to a specific, high demanding, quantum application. Next, a polarization controller (PC) is used to rotate the polarization state of the photon pairs by $45^{\circ}$. An unbalanced Mach-Zehnder interferometer (UMI) is then used in order to engineer polarization entangled state of the form $\frac{1}{\sqrt{2}}\left(|H\rangle|H\rangle+e^{i \phi}|V\rangle|V\rangle\right)$. The UMI is made of two fiber polarizing beam splitters (f-PBS) connected by polarization maintaining fibers (PMF). The photon pairs are then probabilistically separated by a fiber beam splitter (f-BS) and the successful separated paired photons are sent to two separated users Alice and Bob. Each user has a set of entanglement analyzer, comprising a half-wave plate (HWP), a polarizing beam splitter (PBS) and an avalanche photo-diode (APD). The zoomed inset shows the principle of the entanglement preparation stage. Diagonally polarized photon pairs $|D\rangle|D\rangle$ enter the UMI and consequently four possibilities are made available at the output. If a photon pair is separated inside the UMI, we end up with either $|H\rangle|V\rangle$ or $|V\rangle|H\rangle$ possibility. If not, the possibilities are reduced to either $|V\rangle|V\rangle$ or $|H\rangle|H\rangle$, i.e. the contributions to the desired entangled state. 


\subsubsection{Photon pair generation via type-0 SPDC}

As usual, we begin by describing, experimentally, the generation of the photon pairs. Let us take a closer look at Fig.5.1. Here, vertically polarized light at $780.24 \mathrm{~nm}$ from a continuous wave, commercial tapered amplifier system (Toptica Photonics TA pro), is injected into a $4.5 \mathrm{~cm}$ long, home-made type-0 PPLN/W. As a result, co-polarized photon pairs, around the degenerate wavelength of $1560.48 \mathrm{~nm}$, are generated via type-0 SPDC process inside the PPLN/W. The down conversion process can be described by $|V\rangle_{p} \stackrel{\text { type }-0}{\longrightarrow}|V\rangle_{s}|V\rangle_{i}$ where the subscripts $p, s$ and $i$ denote the pump, signal and idler photons, respectively. The desired phase matching condition, i.e. $780.24 \mathrm{~nm} \stackrel{\text { SPDC }}{\longrightarrow} 1560.48 \mathrm{~nm}$, is achieved through the use of a PPLN/W having a width of $7 \mu \mathrm{m}$ along with a poling period of $16.3 \mu \mathrm{m}$. Additionally, the PPLN/W is temperature stabilized at $113.85^{\circ} \mathrm{C}$.

As for the SPDC characterization purposes, we have followed the experimental procedure described in the previous Chapter 3. The resulting temperature dependent emission spectrum from the SPDC characterization for this particular type-0 photon pair generator is presented in Fig.5.2. As can be seen in (a), the wavelength of the signal and the idler components can be tuned over more than $50 \mathrm{~nm}$ in spectral range for a temperature change of $3^{\circ} \mathrm{C}$ to the PPLN/W. Meanwhile in (b), we see a degenerate spectrum at around $1560 \mathrm{~nm}$ when the PPLN $/ \mathrm{W}$ is temperature stabilized at $113.85^{\circ} \mathrm{C}$. The full width at half maximum (FWHM) of the emission bandwidth spectrum is measured to be $32 \mathrm{~nm}$, which corresponds to $4 \mathrm{THz}$.

\subsubsection{Adaptable photon bandwidth via suitable spectral filtering}

Even though our type-0 photon pair generator exhibits a large SPDC emission bandwidth of $4 \mathrm{THz}$, the bandwidth can always be reduced, deliberately, via suitable spectral filtering, so as to match the acceptance bandwidth of any desired quantum applications. Within the scope of this thesis, we aim at demonstrating three spectral filtering solutions such that each solution is unique to a specific, high-demanding, application in quantum communication:

- The use of a standard $80 \mathrm{GHz}$ dense wavelength division multiplexing (DWDM) filter (AC photonics) to address DWDM based QKD protocols [Qi et al. 2010b, Kaiser et al. 2012b, Yoshino et al. 2012, Patel et al. 2014]; 
(a)

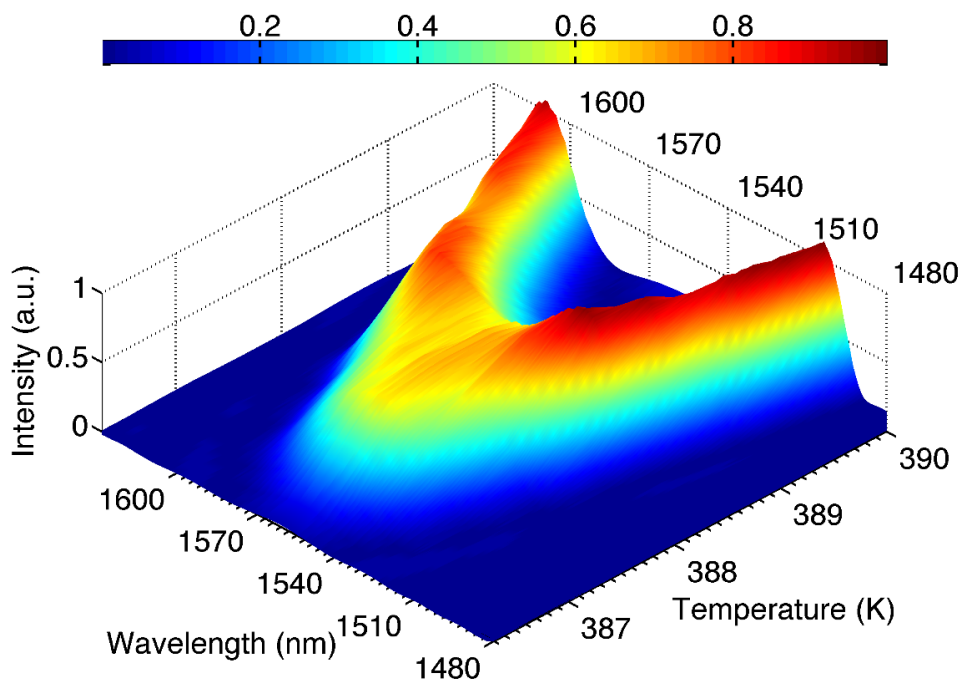

(b)

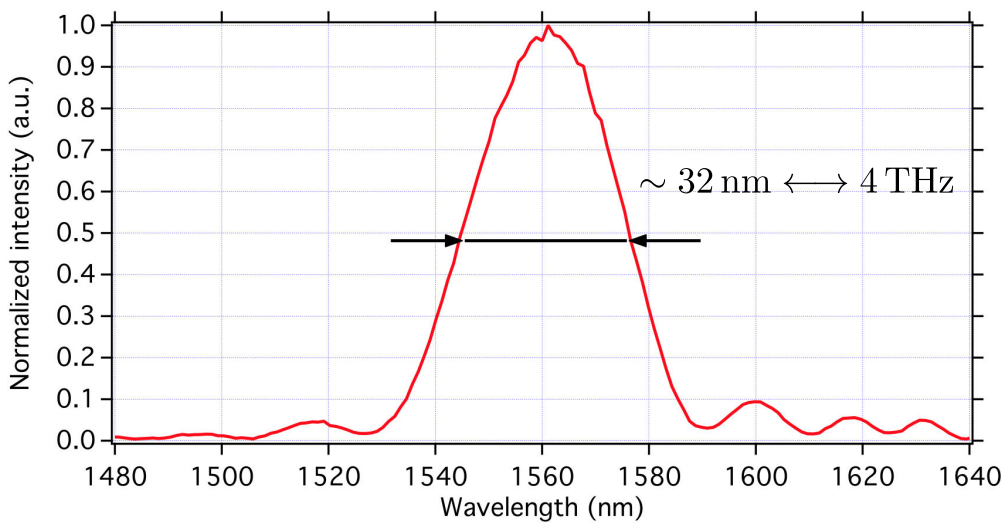

Figure 5.2: (a): 3-dimensional representation concerning the temperature dependent SPDC emission spectrum of our type-0 photon pair generator. The spectral density is visualized over the full phase matching curve as a function of the waveguide temperature. For ease of reading, the intensity is color coded, as indicated by the on-top color bar. From the data, we observe a wavelength tunability of our photon pair generator of more than $50 \mathrm{~nm}$ for a $3^{\circ} \mathrm{C}$ temperature change. (b): For the stabilized temperature of $113.85^{\circ} \mathrm{C}$, degenerate spectrum around $1560 \mathrm{~nm}$ is observed. We note that all of the above measurements are performed by pump light at $780.24 \mathrm{~nm}$ in a $4.5 \mathrm{~cm}$ long PPLN/W having a width of $7 \mu \mathrm{m}$ and a poling period of $16.3 \mu \mathrm{m}$. 
- The use of a $540 \mathrm{MHz}$ phase shifted fiber Bragg grating filter (PSFBG from AOS $\mathrm{GmbH})$ to answer the demand of broad acceptance bandwidth quantum memories, e.g. room temperature atomic vapors or ion doped crystals [Reim et al. 2010, Clausen et al. 2011, Saglamyurek et al. 2011].

- The use of a narrowband $25 \mathrm{MHz}$ PSFBG (Teraxion) which is compatible with the acceptance bandwidth of quantum memories based on cold-atoms and trapped ions [Chanelière et al. 2005b, Black et al. 2005, Choi et al. 2008, Chen et al. 2008, Piro et al. 2009, Choi et al. 2010,Specht et al. 2011,Wang et al. 2011,Bao et al. 2012,Bimbard et al. 2014].

Most of the optical elements of our source are indeed fiber-based, so are the three filters mentioned above. Consequently, a desired filter can be inserted or removed without much hassle, thus bringing huge advantages to the system. First, any additional losses introduced by mode-matching procedures, i.e. in and out fiber coupling can be totally avoided. Secondly, this "plug-and-play"-type filtering configuration can ensure the longterm stability of the system.

The use of a standalone standard DWDM filter is quite convenient as this type of filter is essentially temperature and polarization independent. Therefore, any additional stabilization setup accompanying the use of such filter is deemed unnecessary. The DWDM filter can simply be characterized by sending light from a tunable laser source (e.g. Tunics T100S-HP) to the common input, and the intensity of the transmitted lights can be monitored using a standard optical power meter (e.g. Coherent Fieldmax). As can be seen in Fig.5.3, such a filter exhibits a flat-top transmission bandwidth of about $80 \mathrm{GHz}$.

However, one should pay close attention to the residual birefringence of both PSFBG filters as such filters are capable of associating different polarization modes with the transmitted wavelengths $\left(|H\rangle_{\lambda_{1}}\right.$ and $\left.|V\rangle_{\lambda_{2}}\right)$. The corresponding disadvantage would be the degradation of the expected entanglement quality. Consequently, it is compulsory to position those filters where only co-polarized photons are available. In this case, the best choice is to place these PSFBGs directly after the PPLNW as $|V\rangle_{s}|V\rangle_{i}$ photon pairs are totally immune to such a problem. Moreover, such filters also show relatively strong temperature dependency of the transmission wavelength. As for the $540 \mathrm{MHz}$ PSFBG manufactured by AOS GmbH, 


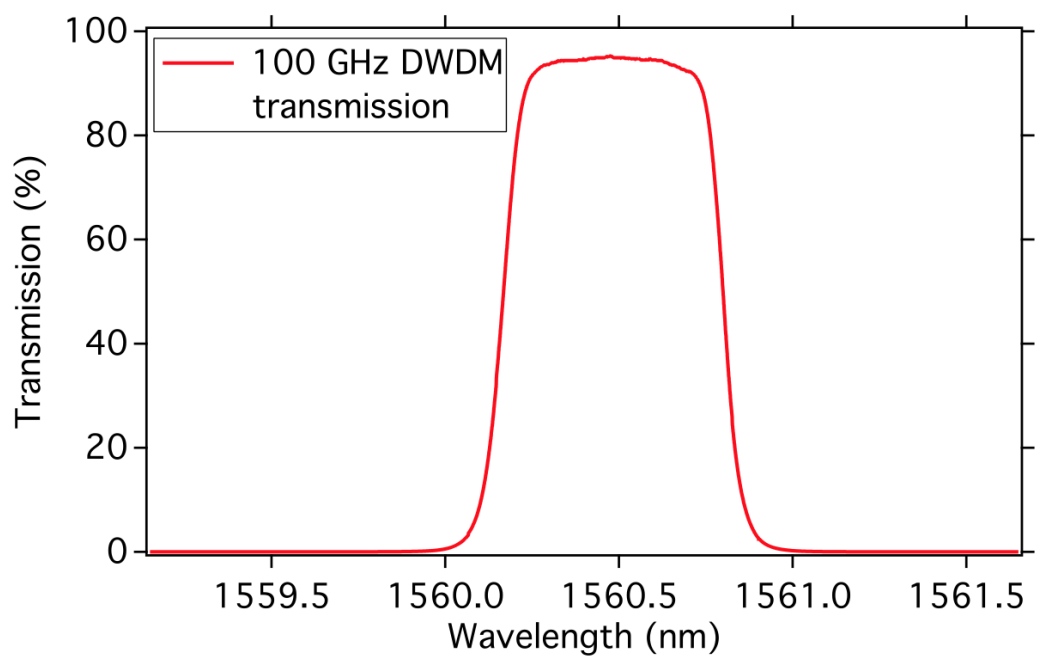

Figure 5.3: The flat-top spectral bandwidth of the $100 \mathrm{GHz}$ DWDM (AC Photonics) filter whose FWHM is measured to be about $80 \mathrm{GHz}$.

strong temperature-wavelength dependency of $\sim 1 \mathrm{GHz} / \mathrm{K}$ is observed. This explains why the filter is shipped in a pre-configured, Peltier element controlled, temperature stabilized box (AOS GmbH UltraBox), allowing the fine tuning of the PSFBG's central wavelength. Meanwhile, the $25 \mathrm{MHz}$ PSFBG from Teraxion somehow exhibits less dependency value compared to $540 \mathrm{MHz}$ one $(\sim 200 \mathrm{MHz} / \mathrm{K})$. Therefore, the Teraxion filter is placed in a home-made temperature stabilization box comprising inexpensive heating elements and a standard PID-type temperature controller (Thorlabs TED200C).

The PSFBG filters are simultaneously characterized and configured with the help of a laser spectroscopy setup (see Fig.5.4 (a)). For this purpose, a tunable diode laser (Toptica Photonics DL100 pro design) is employed. Light from the laser source is routed into a polarization controller $(\mathrm{PC})$ before passing through the desired filter. Eventually, the transmitted power at the output of the filter is monitored. At the same time, a small presplit fraction of the laser light is amplified using an erbium doped fiber amplifier (EDFA) resulting in $100 \mathrm{~mW}$ of power. The amplified light is then up-converted to $780.24 \mathrm{~nm}$ by second harmonic generation (SHG) process in an another home-made PPLN/W. The $3 \mathrm{~mW}$ SHG output then serves to perform the Doppler free saturated absorption spectroscopy in a rubidium cell. The obtained hyperfine structure absorption lines through the spectroscopy serve as absolute frequency references when scanning the laser. Using the above strategy, 
the transmission peaks of both PSFBG filters for vertically polarized light can be fixed at exactly twice the wavelength of the rubidium $|F=2\rangle \rightarrow\left|F^{\prime}=2 \times 3\right\rangle$ hyperfine transition, against which the pump laser is stabilized (see Fig.5.4 (b) and (c). In both figures, the stabilization point of the pump laser is marked by a star).

\subsubsection{Polarization entanglement preparation stage}

As been demonstrated in the previous Chapter 3, the type-II SPDC interaction can be exploited to produce cross-polarized photon pairs, i.e. $|H\rangle_{p} \stackrel{\text { type-II }}{\longrightarrow}|H\rangle_{s}|V\rangle_{i}$ and the polarization entanglement can simply be obtained through appropriate separation and postselection of the photon pairs. As for the type-0 interaction where vertically co-polarized photons are generated, i.e. $|V\rangle_{p} \stackrel{\text { type-0 }}{\longrightarrow}|V\rangle_{s}|V\rangle_{i}$, such straightforward approach is not applicable. In our case, to engineer the polarization entanglement from a type-0 generated photon pairs, an unbalanced Mach-Zehnder (UMI) is employed. Our technique is much more advanced compared to previous realizations [Sanaka et al. 2002, Takesue et al. 2005], as it permits to handle bandwidth as narrow as $25 \mathrm{MHz}$.

The UMI is built upon two fiber polarizing beam splitters (f-PBS) connected together by polarization maintaining fibers (PMF). We begin first by describing the initial state of a photon pair before the UMI,

$$
|\psi\rangle_{\text {init }}=|V\rangle|V\rangle
$$

For simplicity, both photons are considered to be perfectly indistinguishable in all degrees of freedom as we are dealing with degenerate photons which are collected by a single mode fiber. Therefore, common labeling of signal $(s)$ and idler $(i)$ to the photons is unnecessary. Before entering the UMI, the pair is subjected to a polarization rotation by $45^{\circ}$ using a polarization controller (PC) which, in return, transforms the $|V\rangle|V\rangle$ state pair into the diagonal polarization state,

$$
|\psi\rangle_{P C}=\frac{1}{\sqrt{2}}(|V\rangle-|H\rangle) \frac{1}{\sqrt{2}}(|V\rangle-|H\rangle)=|D\rangle|D\rangle .
$$

The pair then encounter the UMI. The UMI is pre-configured such that a delay of $\delta t$ is introduced between two different polarization modes $|H\rangle$ and $|V\rangle$. In this case, the horizon- 
(a)

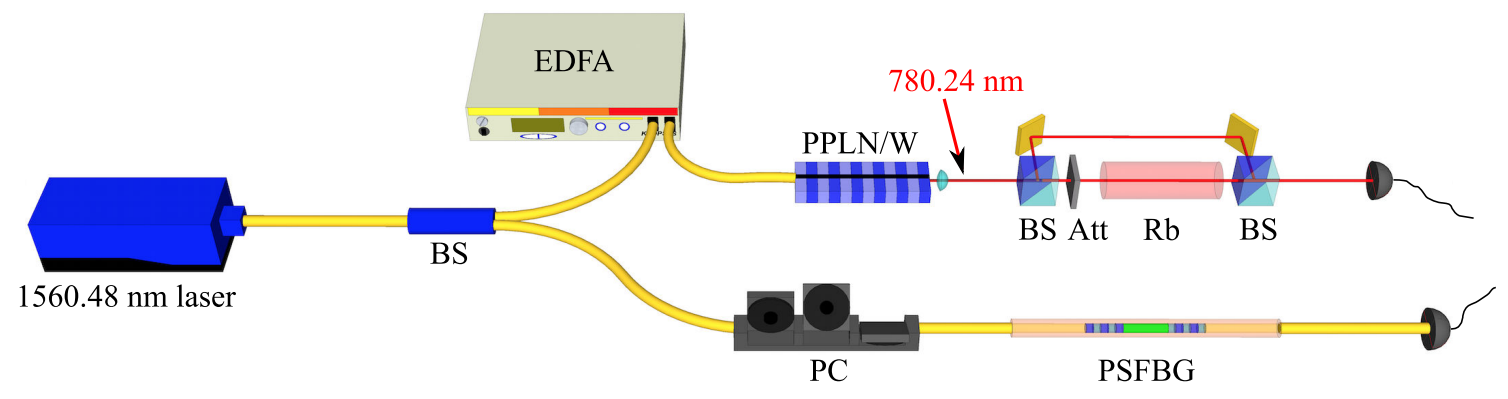

(b)

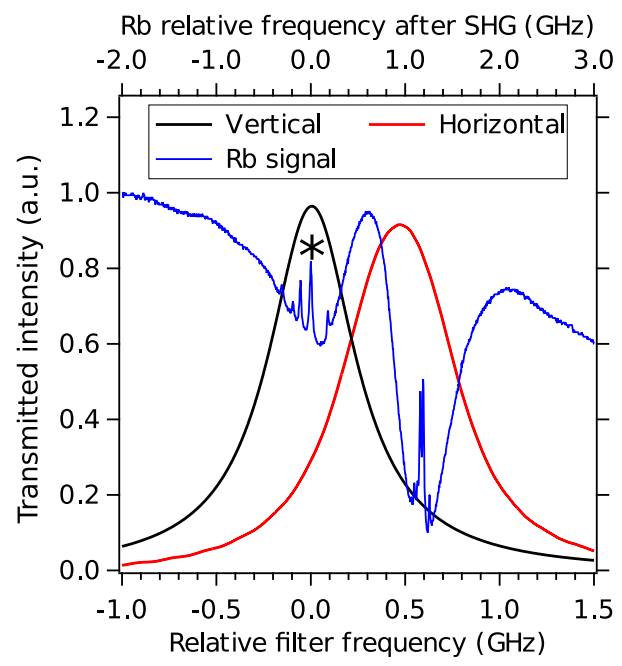

(c)

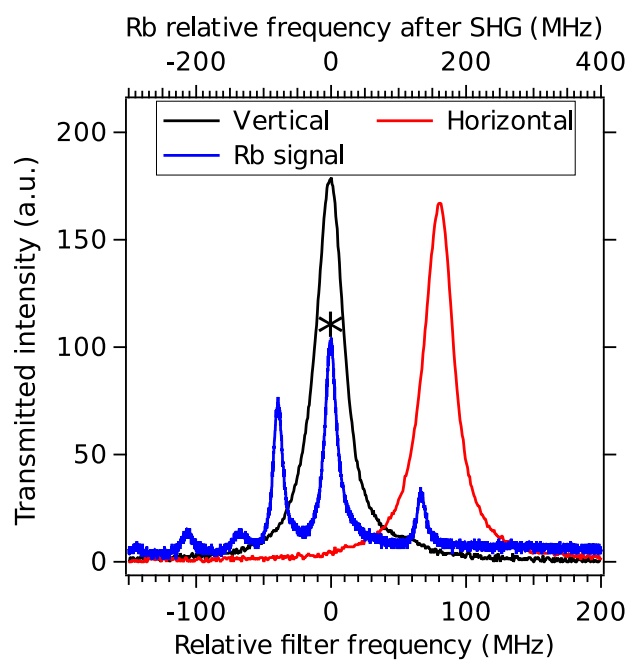

Figure 5.4: (a): Experimental setup toward simultaneous characterization and configuration of the two phase shifted fiber Bragg grating (PSFBG) filters. Optical light around $1560.48 \mathrm{~nm}$ from a tunable laser source is sent through the filter under test. Next, the polarization and wavelength dependent transmission is monitored. A laser spectroscopy setup is also employed in order to obtain an absolute frequency reference. For this purpose, a small fraction of the laser light is amplified by an erbium doped fiber amplifier (EDFA) and subsequently frequency doubled in a PPLN/W. The output light at $780.24 \mathrm{~nm}$ is then sent to a standard rubidium saturated absorption spectroscopy setup. (b) and (c): The Lorentzian transmission spectrum of $540 \mathrm{MHz}$ and $25 \mathrm{MHz}$ PSFBG filters, respectively. 
tally polarized photon is sent to propagate along the short arm while the vertically polarized one is sent into the opposite longer arm of the UMI. These two arms are recombined by another f-PBS where the quantum state of the exiting photon pair reads

$$
|\psi\rangle_{U M I}=\frac{1}{2}\left(|H\rangle_{e}|H\rangle_{e}+e^{i \phi}|V\rangle_{l}|V\rangle_{l}-e^{i \frac{\phi}{2}}|H\rangle_{e}|V\rangle_{l}-e^{i \frac{\phi}{2}}|V\rangle_{l}|H\rangle_{e}\right)
$$

Here, time-binning of the photons' arrival times is applicable due to $\delta t \neq 0$ and it is represented by $e$ ("early") and $l$ ("late') subscripts. Meanwhile, $\phi$ denotes the phase relation between the different arms of the UMI. If the recombined photon pair is subjected to a probabilistic separation by a fiber beam splitter (f-BS) whose outputs are associated with two separated users, namely Alice and Bob, we obtain the following output state of

$$
\begin{aligned}
|\psi\rangle_{B S}= & \frac{1}{4}\left(\left(|H\rangle_{e, a}|H\rangle_{e, a}+|H\rangle_{e, b}|H\rangle_{e, b}+2|H\rangle_{e, a}|H\rangle_{e, b}\right)\right. \\
& +e^{i \phi}\left(|V\rangle_{l, a}|V\rangle_{l, a}+|V\rangle_{l, b}|V\rangle_{l, b}+2|V\rangle_{l, a}|V\rangle_{l, b}\right) \\
& \left.-2 e^{i \frac{\phi}{2}}\left(|H\rangle_{e, a}|V\rangle_{l, a}+|H\rangle_{e, b}|V\rangle_{l, b}+|H\rangle_{e, a}|V\rangle_{l, b}+|H\rangle_{e, b}|V\rangle_{l, a}\right)\right)
\end{aligned}
$$

where the additional $a$ and $b$ subscripts correspond to Alice and Bob, respectively. From Eq.(5.4), if we consider only cases that give rise to coincidences between the two users, we obtain, from the post-selection, the following quantum state:

$$
\begin{aligned}
|\psi\rangle_{B S-p o s t}= & \frac{1}{2}\left(\left(|H\rangle_{e, a}|H\rangle_{e, b}+e^{i \phi}|V\rangle_{l, a}|V\rangle_{l, b}\right.\right. \\
& \left.\left.-e^{i \frac{\phi}{2}}|H\rangle_{e, a}|V\rangle_{l, b}-e^{i \frac{\phi}{2}}|H\rangle_{e, b}|V\rangle_{l, a}\right)\right)
\end{aligned}
$$

after suitable normalization.

From Eq.(5.5), if we further post-select only the events involving simultaneous arrival of the paired photons on the $\mathrm{f}-\mathrm{BS}$, it then reads

$$
|\psi\rangle_{B S-\text { post-post }}=\frac{1}{\sqrt{2}}\left(|H\rangle_{e, a}|H\rangle_{e, b}+e^{i \phi}|V\rangle_{l, a}|V\rangle_{l, b}\right),
$$

after suitable renormalization. 
However, the quantum state described in Eq.(5.6) can only be achieved by post-selection, provided $\delta t$, which is the delay between the two arms of the UMI, is greater than the coherence time of the photons $\tau_{\text {photon }}$ and the timing jitters $\tau_{j i t t e r s}$ of the employed detectors. Moreover, the labeling of "early" and "late" time-bins is valid, if and only if, the photon pair creation time is known. In the case of SPDC pumped with continuous laser, it is practically impossible to differentiate between those two time-bins thus the labeling of $e$ and $l$ can be completely dropped [Shih \& Alley 1988,Franson 1989, Brendel et al. 1992,Kwiat et al. 1993]. To actually observe entanglement, the phase relation $\phi$ between the two contributions of the state $|\psi\rangle_{B S-\text { post-post }}$ should stay constant, i.e stable, at least throughout the total duration of the experiment. In this regard, a constant $\phi$ can be preserved if the coherence time of the pump laser $\tau_{\text {pump }}$ is greater than $\delta t$, thus permitting the following polarization entangled state to be obtained:

$$
|\psi\rangle_{\text {final }}=\frac{1}{\sqrt{2}}\left(|H\rangle_{a}|H\rangle_{b}+e^{i \phi}|V\rangle_{a}|V\rangle_{b}\right)
$$

where, in overall, the inequality $\tau_{\text {pump }} \gg \delta t \gg \tau_{\text {photon }}+\tau_{\text {jitters }}$ is satisfied.

With our source, it is possible to prepare any arbitrary superposition of the maximally polarization entangled Bell states $\left|\Phi^{+}\right\rangle$and $\left|\Phi^{-}\right\rangle$. The latter can be achieved simply by manipulating the phase difference between the two arms of the UMI. Let us consider the following two-photon state available at the input of the UMI:

$$
|\psi\rangle_{\text {init }}=\left(\alpha_{s}|H\rangle_{s}+\beta_{s}|H\rangle_{s}\right)\left(\alpha_{i}|V\rangle_{i}+\beta_{i}|V\rangle_{i}\right)
$$

where the subscripts $s$ and $i$ denote signal and idler photons, respectively, and the normalization of $|\alpha|_{s, i}^{2}+|\beta|_{s, i}^{2}=1$ is satisfied. After appropriate post-selection involving only the cases of simultaneous arrival of paired photons, the following non-maximally polarization entangled Bell state is obtained:

$$
|\psi\rangle_{\text {post }}=\frac{1}{N}\left(\alpha^{2}|H\rangle_{s}|H\rangle_{i}+e^{i \phi} \beta^{2}|V\rangle_{s}|V\rangle_{i}\right)
$$

where the normalization $N=\sqrt{\alpha^{4}+\beta^{4}}$. Interestingly, we can also prepare non-maximally entangled states as the superposition of the other Bell states namely $\left|\Psi^{+}\right\rangle$and $\left|\Psi^{-}\right\rangle$simply 
by rotating the polarization of one photon that is successfully separated by the f-BS with the help of a half-wave plate.

\subsubsection{Satisfying $\tau_{\text {pump }} \gg \delta t \gg \tau_{\text {photon }}+\tau_{\text {jitters }}$}

The requirement $\tau_{\text {pump }} \gg \delta t \gg \tau_{\text {photon }}+\tau_{\text {jitters }}$ should be experimentally fulfilled in order to observe the entangled state $|\psi\rangle_{\text {final }}=\frac{1}{\sqrt{2}}\left(|H\rangle_{a}|H\rangle_{b}+e^{i \phi}|V\rangle_{a}|V\rangle_{b}\right)$. Let us assume the most extreme case where the generated photons are spectrally filtered by the ultra-narrowband $25 \mathrm{MHz}$ PSFBG filter. Correspondingly, the single photon coherence time $\tau_{\text {photon }}$ is expected to be $\sim 18 \mathrm{~ns}$, which is much greater compared to the timing jitters of typical indium-gallium-arsenide (InGaAs) avalanche photo-diodes (APD) specifically used for this experiment, $\tau_{j i t t e r s} \sim 200 \mathrm{ps}$. Therefore, $\tau_{j i t t e r s}$ contribution can be considered negligible. Meanwhile, the delay $\delta t$ is fixed at $\sim 76 \mathrm{~ns}$ which is equivalent to $18 \mathrm{~m}$ of path length difference between the two PMFs of the UMI. To this extent, the requirement of $\delta t \gg \tau_{\text {photon }}$ is fulfilled.

In the case of non-stabilized diode laser sources, it is typically difficult to maintain $\tau_{\text {pump }}$ over more than $100 \mathrm{~ns}$ in the long run, thus representing a major obstacle toward fulfilling $\tau_{\text {pump }} \gg \delta t$ requirement. However, $\tau_{\text {pump }} \gg \delta t$ can be greatly increased by stabilizing the pump laser. It is for this particular reason why we actually opted for $780.24 \mathrm{~nm}$ as the pump wavelength. At such wavelength, the pump laser can be conveniently locked to the $\mathrm{D}_{2}$ line of rubidium by means of a laser spectroscopy setup. For this particular purpose, a $\mathrm{mW}$ fraction of the laser light is directed toward a second set of saturated absorption spectroscopy setup. In our case, the stabilization is made on the $|F=2\rangle \rightarrow\left|F^{\prime}=2 \times 3\right\rangle$ hyperfine crossover transition of the $\mathrm{D} 2$ line of ${ }^{87} \mathrm{Rb}$. By using a small electromagnet, the Zeeman shifted resonance is modulated by $\sim 20 \mathrm{kHz}$. The saturation absorption signal is then demodulated using a lock-in amplifier (EG\&G 5210), and fed forward to the laser diode current through a home-made PID controller. The speed of the stabilization system is sufficient in order to achieve a laser linewidth $<150 \mathrm{kHz}$, over all the relevant time scales, which guarantees $\tau_{\text {pump }}>3 \mu \mathrm{s}$, therefore $\tau_{\text {pump }} \gg \delta t$. Overall speaking, our system now fulfills $\tau_{\text {pump }} \gg \delta t \gg \tau_{\text {photon }}$. 


\subsubsection{Phase stabilization}

For high-quality polarization entanglement demonstration via the violation of the BellCHSH inequality, the phase relation $\phi$ between $|H\rangle|H\rangle$ and $|V\rangle|V\rangle$ contributions of the desired entangled state needs to be kept stable throughout the full measurement time. This simply translates to a constant phase requirement between the short and the long arms of the UMI. In our case, the variation of the phase comes, notably from the surrounding thermal fluctuations affecting the polarization maintaining fibers (PMF) of the UMI. As a result, the refractive index of such fibers also fluctuates. It has been shown that in order to observe near perfect entanglement visibility with the Bell-CHSH inequality test, the phase fluctuation $\Delta \phi$ should be kept below $1 \%$, implying the following condition of $\Delta \phi<\frac{2 \pi}{50}$.

Concerning previous works, standard temperature stabilization system acting on the employed interferometer should deliver enough stability to the phase relation $\phi$ where the path length difference between both arms of the interferometer is on the order of a few tens of centimeters [Tanzilli et al. 2002, Sanaka et al. 2002, Takesue et al. 2005, Pomarico et al. 2009]. To give a basic idea, by implementing a standard, off-the-shelf stabilization system, a stability of about $10 \mathrm{mK}$ can be expected. Therefore, such system is capable to handle the temperature stabilization concerning a path length difference of the interferometer up to $400 \mathrm{~cm}$ while maintaining near $100 \%$ entanglement visibility. However, our experiment requires a much more capable system as we are dealing with photons exhibiting ultra-long coherence time in the extreme scenario. To accommodate such coherence times, we recall that the path length difference of our interferometer is of about $18 \mathrm{~m}$, such that the use of a temperature stabilization system featuring sub-mK stability is mandatory.

To avoid the latter constraint, we have decided to implement an active phase stabilization system for the UMI. In this case, the system relies on the continuous information on the accumulated phase inside the UMI. Thanks to such information, constant correction of the PMF's optical length can be applied via a home-made piezoelectric (PZT) fiber stretcher acting on the long arm of the UMI so as to reduce, to the minimum, the temperature induced phase fluctuations. As can be seen from Fig.5.5, a telecom diode laser source (Toptica Photonics DL100 pro design) is employed to emit low-power ( $\leq 100 \mathrm{nW})$ reference light at $1558.6 \mathrm{~nm}$, i.e. slightly shifted from the wavelength of the actual photon pairs so 


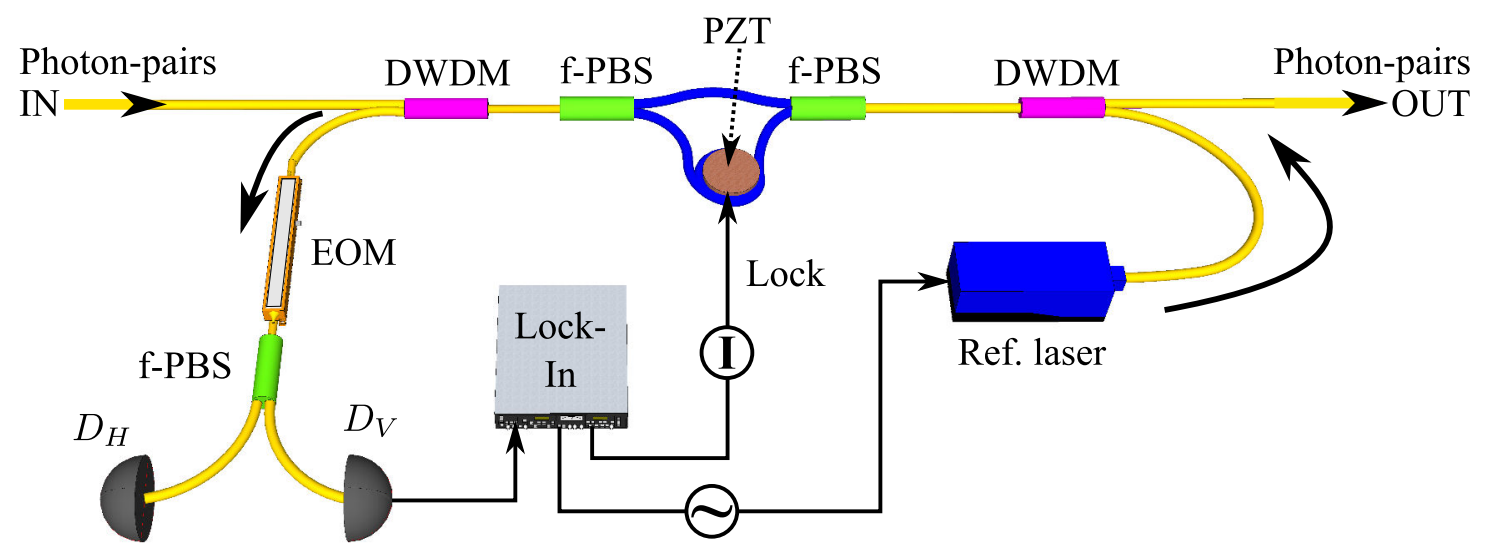

Figure 5.5: Implementation of the phase stabilization system of the UMI, i.e. entanglement preparation interferometer. The system relies on the information of the accumulated phase by a counter-propagating reference laser light in the UMI. Depending on the measured phase, a correction signal is sent to a home-made piezoelectric (PZT) fiber stretcher. Moreover, the phase inside the UMI can be accurately tuned with the help of an electro-optic modulator (EOM).

as to avoid noise background due to scattering in the optical setup. The reference light is then diagonally polarized and sent into the UMI via a $200 \mathrm{GHz}$ ITU 21/23 DWDM filter, i.e. through wavelength multiplexing. Note that the reference light is sent in the counterpropagative direction compared to that of the photon pairs at $1560.48 \mathrm{~nm}$. At the output of the UMI, the polarization state of the reference light reads

$$
|\psi\rangle_{r}=\frac{1}{\sqrt{2}}\left(|H\rangle_{r}+e^{i \phi_{r}}|V\rangle_{r}\right)
$$

where $\phi_{r}$ denotes the relative phase difference between the short (horizontal) and long (vertical) arms of the UMI. As a side note, the subscript $r$ represents every elements associated with the reference laser light. The reference light is again separated from main experimental setup via another identical DWDM filter, i.e. through wavelength demultiplexing. After the separation, light passes then through an electro-optic modulator (EOM). Up to this point, the previous polarization state evolves into

$$
|\psi\rangle_{E O M}=\frac{1}{\sqrt{2}}\left(|H\rangle_{r}+e^{i\left(\phi_{r}+\phi_{E O M}\right)}|V\rangle_{r}\right)
$$


where $\phi_{E O M}$ represents the manipulable artificial phase added by the EOM to the vertical contribution. Then the polarization state of the reference light is rotated by $45^{\circ}$ using a polarization controller (not shown in Fig.5.5 for simplicity reason) resulting in the following state:

$$
|\psi\rangle_{P C}=\frac{1-e^{\left(\phi_{r}+\phi_{E O M}\right)}}{2}|H\rangle_{r}+\frac{1+e^{\left(\phi_{r}+\phi_{E O M}\right)}}{2}|V\rangle_{r}
$$

A fiber beam splitter (f-PBS) is employed to separate the $|H\rangle_{r}$ and $|V\rangle_{r}$ modes. Eventually, light intensities $I_{H}$ and $I_{V}$ representing the accumulated phase fluctuations can be conveniently measured by two detectors $D_{H}$ and $D_{V}$, respectively, where

$$
\begin{gathered}
I_{H} \propto \sin ^{2}\left(\frac{\phi_{r}+\phi_{E O M}}{2}\right) . \\
I_{V} \propto \cos ^{2}\left(\frac{\phi_{r}+\phi_{E O M}}{2}\right) .
\end{gathered}
$$

Consequently, the detected intensity by $D_{V}$ permits inferring the phase term $\left(\phi_{r}+\right.$ $\left.\phi_{E O M}\right) \bmod 2 \pi$.

Overall, our phase stabilization scheme involves a standard feedback loop configuration. We begin with sending a $32 \mathrm{kHz}$ modulation signal originated from the lock-in amplifier to the modulation input of the reference laser diode resulting in the modulation of the reference wavelength. From Eqs.(5.14) and (5.14), it is obvious that the recorded intensities by both detectors $D_{H}$ and $D_{V}$ are wavelength independent, but depends only on the combined phase fluctuations. Therefore, the intensity, for instance $I_{V}$, is measured using a fast photodiode (Thorlabs DET10C), labeled $D_{V}$. The voltage output from such detector is sent back to lock-in amplifier in order to be demodulated. In the meantime, the incoming signal is multiplied with the $32 \mathrm{kHz}$ modulation signal and sent to a low-pass filtering. As a result, an error signal is obtained which corresponds to the first derivative of intensity $I_{V}$. Concerning the error signal, it needs to be kept always at zero level. In order to achieve such a phase correction, the error signal is sent to the PZT via an integrator circuit that guarantees to constantly keep the phase term $\phi_{r}+\phi_{E O M}$ at zero. Interestingly, an accurate tuning of $\phi_{r}$, which represents the phase inside the UMI, can be conveniently achieved simply by adjusting $\phi_{E O M}$. Furthermore, the response time of our system toward correcting the phase fluctuations is quite fast, i.e. on the order of $1 \mathrm{~ms}$ which is mainly limited by the response 
time of the PZT.

To ensure an overall long term stability of our system, we have employed the transfercavity lock configuration. In this case, the reference laser diode at $1558.6 \mathrm{~nm}$ is stabilized upon a cavity where the cavity itself is locked to the primary pump laser at $780.24 \mathrm{~nm}$. As a result, our system is capable to achieve an overall phase stability better than $\frac{\pi}{100}$ over several days.

\subsubsection{Filter-associated coincidence histogram}

Most of the experimental requirements are now fulfilled. Here, we are interested in observing the coincidence histogram associated with each filter configuration. The experimental setup allowing such observation is illustrated in Fig.5.6. As has been discussed before, it is imperative for the filters, notably the phase shifted fiber Bragg grating (PSFBG) ones, to be positioned in front of the entanglement preparation stage, i.e. UMI, so as to avoid any polarization dependent transmission. Moreover, as we are dealing with degenerate photon pairs, a fiber beam splitter (f-BS) is employed for probabilistic separation of the pairs. The truly separated photon pairs are then shared by Alice and Bob, who both use a free-running, InGaAs APD (IDQ 220) to detect the incoming photons. Each APD exhibits a quantum detection efficiency of $20 \%$ and a dark count probability of $10^{-6} /$ ns. The coincidence rate between the two APDs is processed by a coincidence counter (\&).

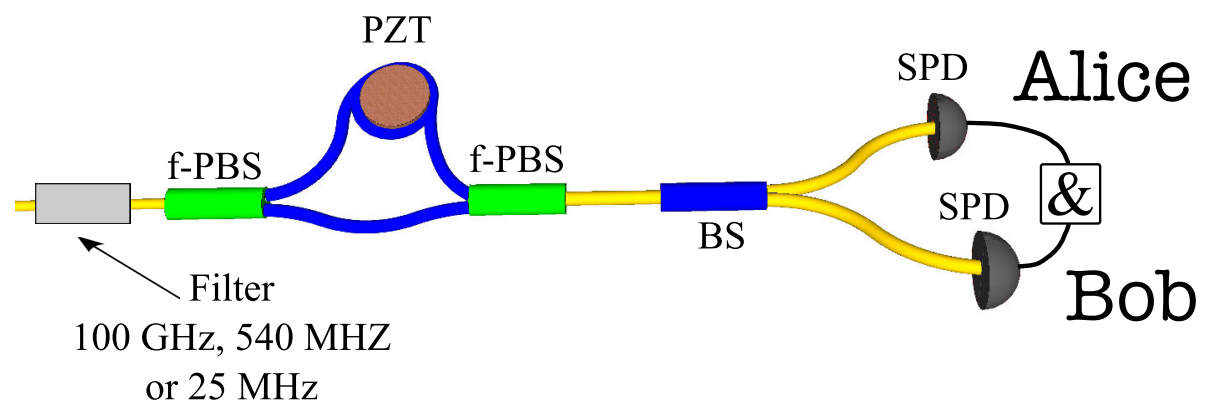

Figure 5.6: Representation of the experimental setup toward observing the coincidence histogram associated with three different filtering solutions. Once correlated paired photons are made available after the entanglement preparation stage (UMI), they are then probabilistically separated by a fiber beam splitter (f-BS). The separated photons are eventually detected by Alice and Bob. The coincidence is then processed by a coincidence counter $(\&)$ thus revealing different arrival times of the paired photons. 
As can be seen in Fig.5.7, the difference in delay $\delta t$ between two arms of the UMI is long enough to resolve the three separated coincidence peaks for all three filters. The outer peaks at \pm 76 ns contain the the cross-polarized photon pair contributions, i.e. pairs that have been separated inside the UMI. Meanwhile, the central peak at zero delay is twice as high as the outer ones as it contains the desired contributions of $|H\rangle|H\rangle$ and $|V\rangle|V\rangle$ to the entangled state. We note that only the contributions at zero delay lead to the expected entangled state described in Eq.(5.7). To observe such state, a post selection in the time domain has therefore to be applied thanks to a suitable time window that can be easily adjusted in position and duration with the coincidence counter. For the latter, a time-to-amplitude converter (Ortec 567) featuring single channel analyzing capability is employed.

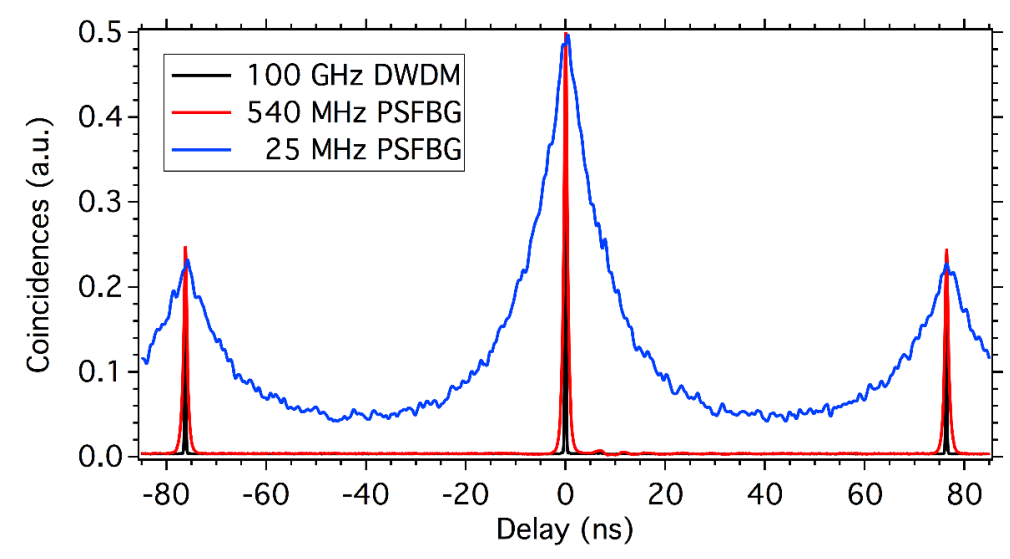

Figure 5.7: Coincidence histogram associated with all three filters. Three well separated coincidence peaks are observed on all occasions. This is a clear and valid confirmation for the chosen $\delta t$ of $76 \mathrm{~ns}$ thus allowing appropriate entanglement post-selection of $|V\rangle|V\rangle$ and $|H\rangle|H\rangle$ contributions in the central peak.

For the $80 \mathrm{GHz}$ DWDM filter, a photon coherence time of $5 \mathrm{ps}$ is expected. Therefore, the width of the observed coincidence peak associated with the DWDM filter is solely defined by the convolution of the detectors' timing jitters. In this case, the width of the coincidence peak is measured to be $230 \pm 2 \mathrm{ps}$ which leads to a timing jitter of $230 \mathrm{ps} / \sqrt{2}=163 \mathrm{ps}$ for a single detector (considering the Gaussian shaped profile), which is in good agreement with the manufacturer's specification. Meanwhile, the implementation of both PSFBG filters results in the broadening of the coincidence peaks reflecting the increase of the photons' coherence time. For the $540 \mathrm{MHz}$ and the $25 \mathrm{MHz}$ PSFBG filters, the respective coincidence peak widths are measured to be $800 \pm 20$ ps and $15.6 \pm 0.7 \mathrm{~ns}$, respectively, which are in good 
agreement with the corresponding filter specifications.

\subsubsection{Entanglement analysis}

The main aim of this subsection is to verify the level of the entanglement quality contained in the produced entangled state via the violation of the Bell-CHSH inequality for all three filter configurations. However, we first begin with the demonstration of our capability to accurately tune the phase inside the UMI to either 0 or $\pi$.

We note that all of the measurements conducted in this subsection follow the experimental setup shown in Fig.5.8.

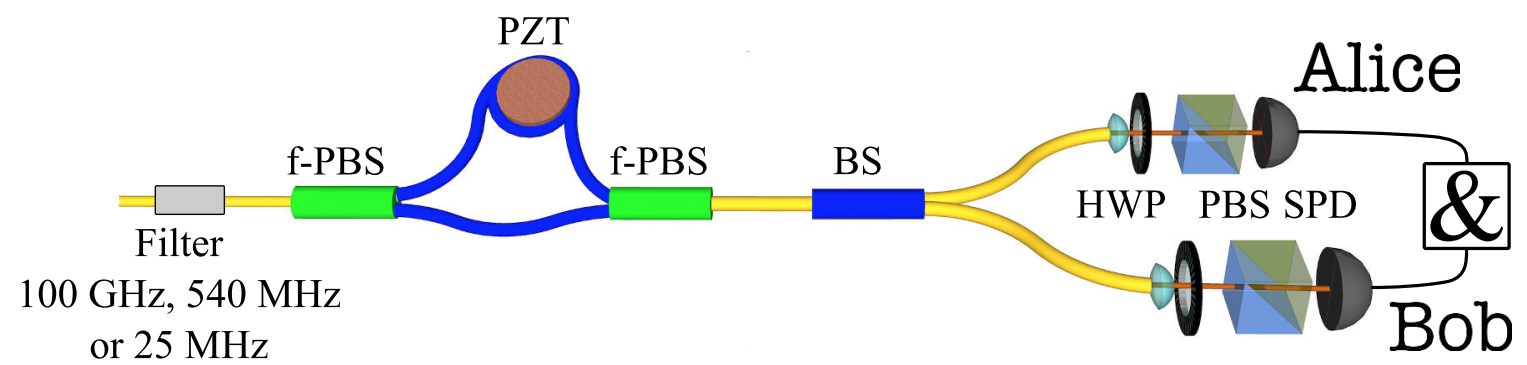

Figure 5.8: Experimental setup toward demonstrating the phase tuning capability as well as the violation of the Bell-CHSH inequality. As of now, Alice and Bob install, on their respective side, a set of entanglement analyzers comprising a half-wave plate (HWP), a polarizing beam splitter (PBS) and an avalanche photo-diode (APD).

\subsubsection{Phase tuning capability}

As a first measurement, the phase tuning capability is demonstrated. To this point, it is assumed that the general entangled quantum state of $|\psi\rangle_{\text {final }}=\frac{1}{\sqrt{2}}\left(|H\rangle_{a}|H\rangle_{b}+e^{i \phi}|V\rangle_{a}|V\rangle_{b}\right)$ is shared between two users Alice and Bob. As for now, they both fix their respective halfwave plates at $22.5^{\circ}$, thus projecting the entangled state into the phase sensitive diagonal basis $\{|D\rangle,|A\rangle\}$. In the meantime, a control signal in the form of a continuous voltage ramp is sent to the home-made piezo-electric (PZT) fiber stretcher acting on the long arm of the UMI. The resulting coincidence rate between Alice's and Bob's respective detectors is recorded as a function of the applied voltage on the PZT, therefore as a function of the phase $\phi$ set in the UMI. These measurements are repeated for all three filters.

The observed sinusoidal oscillation of the coincidence rate for all filter bandwidths is 
shown in Fig.5.9. Correspondingly, such behavior demonstrate the generation of the maximally entangled polarization Bell states, being $\left|\Phi^{+}\right\rangle$for $\phi=0$, and $\left|\Phi^{-}\right\rangle$for $\phi=\pi$. We observe raw visibilities of $99.9 \pm 1.2 \%, 97.1 \pm 1.2 \%$, and $88 \pm 2 \%$ associated with $100 \mathrm{GHz}$, $540 \mathrm{MHz}$ and $25 \mathrm{MHz}$ filter bandwidths, respectively. When subtracting the accidental coincidence events, the computed net visibilities are $99.9 \pm 1.2 \%, 99.4 \pm 1.5 \%$, and $97 \pm 2 \%$, respectively. Note that the corresponding visibilities for $25 \mathrm{MHz}$ filter are inferior compared to the other two filtering cases. As ultra-long coherence time photons are concerned, longer time windows are required for the entanglement post-selection, thus increasing the probability of detecting coincidence dark count events. Consequently, the signal-to-noise ratio (SNR) of the measurement is reduced.

To conclude, we stress that the curves shown in Fig.5.9 are already the signature that we have successfully prepare maximally entangled state $|\psi\rangle=\frac{1}{\sqrt{2}}\left(|H\rangle|H\rangle+e^{i \phi}|V\rangle|V\rangle\right)$. As a side note, the type of measurement we have been discussing here, i.e. where the phase of the entangled state is turned continuously, somehow correspond to what is done in energy-time entanglement based demonstration [Tanzilli et al. 2002].

For the upcoming measurement which is the violation of the Bell-CHSH inequality, we deliberately set the phase $\phi$ at $\pi$.

\subsubsection{Violation of Bell-CHSH inequality}

As for now, the maximally polarization entangled Bell state $\left|\Phi^{-}\right\rangle$is produced by our source. Alice now fixes her HWP in four different standard settings, $0^{\circ} \rightarrow|H\rangle, 22.5^{\circ} \rightarrow|D\rangle, 45^{\circ} \rightarrow$ $|V\rangle$ and $-22.5^{\circ} \rightarrow|A\rangle$. For each setting, the coincidence rate is recorded as a function of the continuous rotation of Bob's HWP angle. Note that, for the measurements corresponding to $100 \mathrm{GHz}$ and $540 \mathrm{MHz}$ filter bandwidths, Alice and Bob each employs a free-running InGaAs APD (idQuantique id220) for single photon detection. For the measurement involving the $25 \mathrm{MHz}$ filter, the previous APDs are replaced by two superconducting single photon detectors (SSPD, Scontel TCORPS-001). Even though both SSPDs exhibits a bit lower quantum detection efficiency of $\sim 7 \%$ at $1560 \mathrm{~nm}$, resulting in lower coincidence counts, they however produce less than 10 dark counts per second which in return increases the SNR.

The corresponding correlation curves associated with three different filters bandwidths 


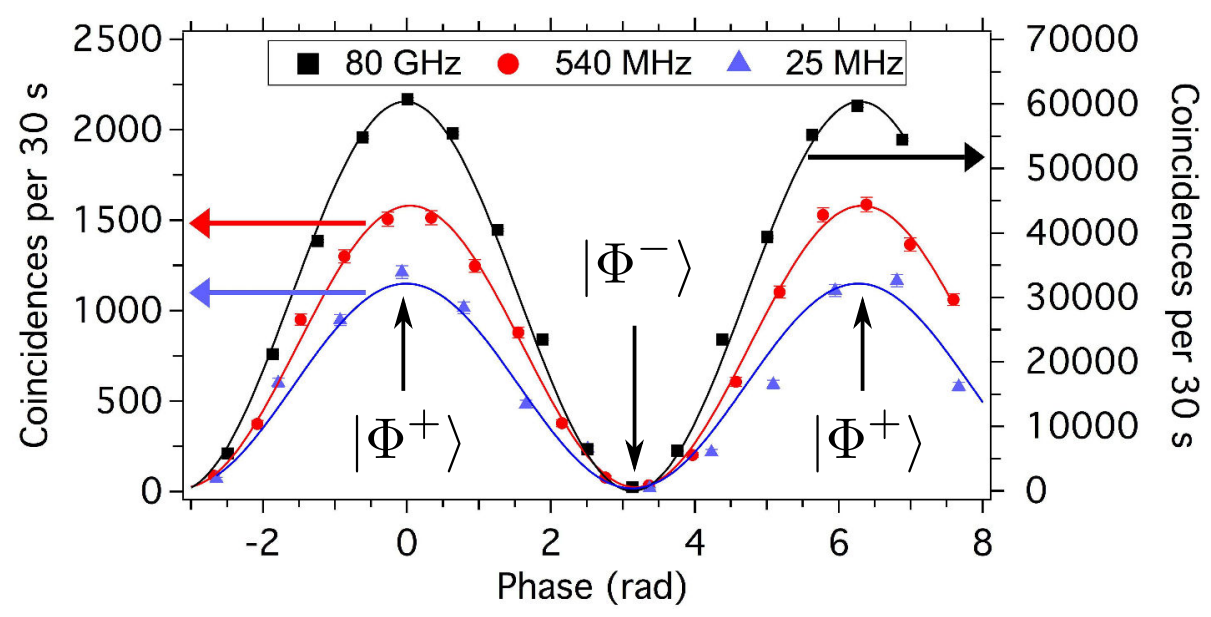

Figure 5.9: Results obtained through the demonstration of phase tuning capability. The measurement consists in fixing both Alice's and Bob's respective HWP to 22.5 thus, preparing their analyzers in the phase sensitive diagonal basis. The coincidence rate between Alice and Bob is then recorded as a function of the phase $\phi$ set in the UMI. We observe, for all filter options, high visibility coincidence rate oscillations thus validating our approach toward achieving high phase stability. As a side note, the computed visibility for $25 \mathrm{MHz}$ filter is a bit lower compared to the other two cases due to lower SNR.

are shown in Fig.5.10. We observe raw visibilities of $99.6 \pm 1.3 \%, 97.1 \pm 0.9 \%$, and $99 \pm 3 \%$ for $100 \mathrm{GHz}, 540 \mathrm{MHz}$ and $25 \mathrm{MHz}$ filter bandwidths, respectively, where such visibilities easily exceed the non-local threshold of $71 \%$ thus proving the high-quality of the expected polarization entanglement. Alternatively, we computed the associated raw $S$ parameters to be $2.82 \pm 0.01,2.80 \pm 0.02$, and $2.82 \pm 0.02$, respectively. These values represent violations of the Bell-CHSH inequality by at least 40 standard deviations. This further highlights the high-quality of the polarization entanglement produced by our source.

\subsubsection{Source's performances}

In addition to high entanglement qualities observed through the violation of the Bell-CHSH inequality, the brightness of the photon-pair generator represents one of its key features, since it defines the rate at which a quantum protocol can be operated. In the previous Chapter 4, we have described extensively the experimental procedure on how to conveniently estimate the brightness $B$ and the SPDC efficiency of the photon pair generator (see Eqs.(4.26) to (4.35)). Based on the same procedure (see Sec.4.2.6), we calculate from 
Legend for all insets

Alice at: $\square\{\mathrm{B}\} \quad \bullet \quad\{\mathrm{V}\} \quad \triangle \quad\{\mathrm{D}\} \quad \nabla \quad\{\mathrm{A}\} \triangleleft$ Noise

(a) $100 \mathrm{GHz}$ DWDM filter

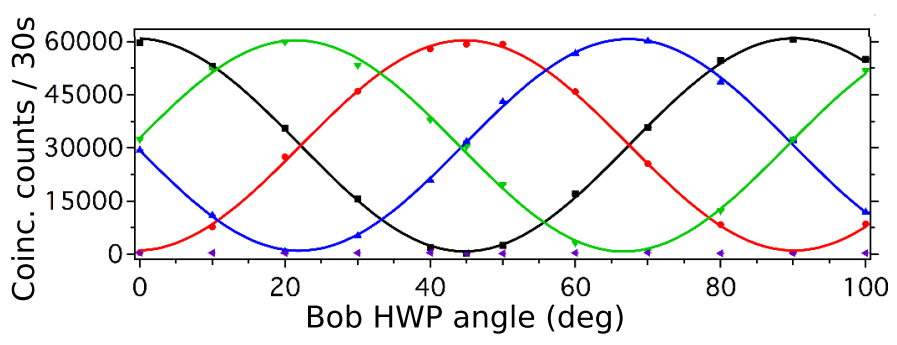

(b) $540 \mathrm{MHz}$ PSFBG filter

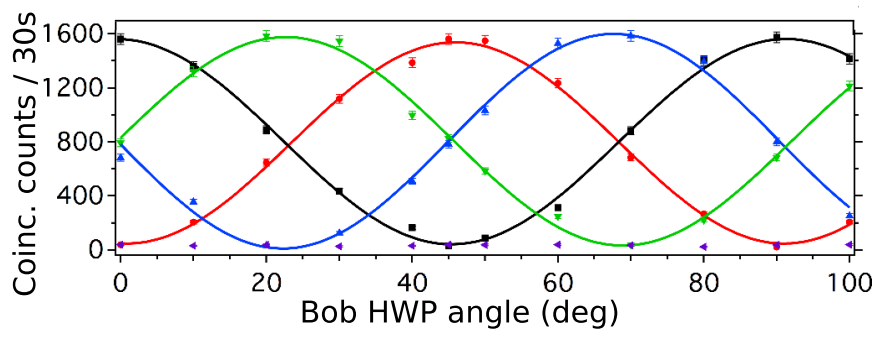

(c) $25 \mathrm{MHz}$ PSFBG filter

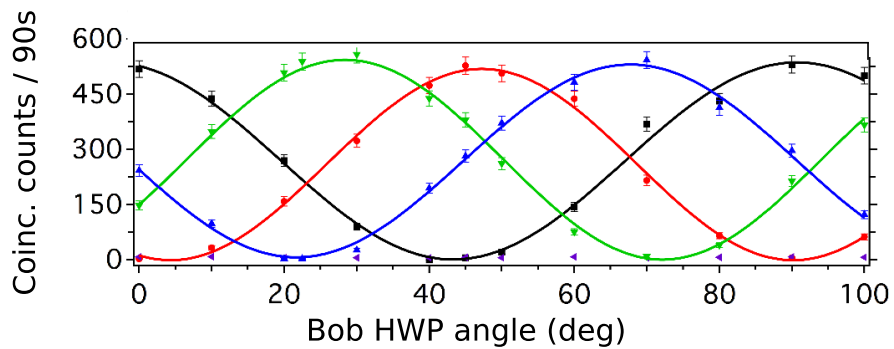

Figure 5.10: Violation of the Bell-CHSH inequality. (a), (b) and (c): Near perfect visibilities of the correlation curves are observed for filter bandwidths of $100 \mathrm{GHz}, 540 \mathrm{MHz}$, and $25 \mathrm{MHz}$, respectively. We note that, thanks to the use of ultra-low noise SSPDs, the SNR for the measurement concerning the $25 \mathrm{MHz}$ is improved such that the achieved high visibility is on par with the other two cases. 
Table 5.1: Summary of the relevant figures of merit concerning our versatile polarization entanglement source for the three filter configurations. All values correspond to raw values, where noise contribution is taken into account. $\frac{\bar{n}}{\tau_{\text {photon }}}$ : mean number of generated photon pairs per coherence time, $V_{\text {raw }}$ : raw entanglement visibility, $\mathcal{F}_{\text {raw }}{ }^{\left|\Phi^{-}\right\rangle}$: fidelity to the closest maximally entangled Bell state, $S_{\text {raw }}$ : the computed Bell parameter from the raw data. Note that the brightness $B$ is defined as the expected amount of photon pairs $\left(N_{\text {pair }}\right)$ being generated per $\mathrm{mW}$ of pump power, per $1 \mathrm{~s}$ of integration time, and per $\mathrm{MHz}$ of spectral bandwidth. The fidelity $\mathcal{F}_{\text {raw }}\left|\Phi^{-}\right\rangle$is obtained by computing $\frac{1+V_{\text {raw }}}{2}$.

\begin{tabular}{|l|l|l|l|}
\hline Bandwidth $(\mathrm{MHz})$ & $80 \cdot 10^{3}$ & 540 & $\mathrm{c}$ \\
\hline Photon coherence time $\tau_{\text {photon }}(\mathrm{ns})$ & $5.5 \cdot 10^{-3}$ & 0.803 & 15.6 \\
\hline PPLN/W coupled pump power $(\mathrm{mW})$ & 0.009 & 0.2 & 3.5 \\
\hline$\frac{\bar{n}}{\tau_{\text {photon }}}\left(\mathrm{ns}^{-1}\right)$ & $5 \cdot 10^{-5}$ & $2 \cdot 10^{-3}$ & $2 \cdot 10^{-2}$ \\
\hline Detected pair rate $\left(\mathrm{s}^{-1}\right)$ & 2000 & 50 & 6 \\
\hline Heralding efficiency (\%) & 13 & 4 & 5 \\
\hline Brightness $B$ & 960 & 300 & 380 \\
\hline$V_{\text {raw }}$ & $99.6 \pm 1.3$ & $97.1 \pm 0.9$ & $99 \pm 3$ \\
\hline $\left.\mathcal{F}_{\text {raw }}{ }^{-}\right\rangle$ & 0.998 & 0.985 & 0.995 \\
\hline$S_{\text {raw }}$ & $2.82 \pm 0.01$ & $2.80 \pm 0.02$ & $2.82 \pm 0.02$ \\
\hline
\end{tabular}

the outcomes, a brightness $B$ of

$$
B=\frac{4.6 \cdot 10^{6}}{\mathrm{~mW} . \mathrm{s} \cdot \mathrm{GHz}}
$$

for our type-0 photon pair generator. Concurrently, the SPDC efficiency is calculated to be

$$
\eta_{S P D C}=4.8 \cdot 10^{-6} \frac{\text { pairs }}{\text { pump photon }} .
$$

We also provide a summary of the relevant figures of merit concerning our versatile polarization entanglement source for the three filter configurations. The summary can be found in Tab.5.1. Finally, Tab.5.2 presents a detailed comparison of our source performances for the three utilized filters to other pertinent and concurrent realizations of ultra-narrow bandwidth photon pair entanglement sources found in the literature. 
Table 5.2: Experimental results for different realizations of ultra-narrow bandwidth photon pair entanglement sources. Note that the specified brightness $B$ values are defined as the expected amount of photon pairs $\left(N_{\text {pair }}\right)$ being generated per $\mathrm{mW}$ of pump power, per $1 \mathrm{~s}$ of integration time, and per $\mathrm{MHz}$ of spectral bandwidth. Also, BW. stands for the photon's bandwidth, $\lambda$ denotes the operation wavelength of the generated photon pairs, and $V_{\text {net }}$ depicts net entanglement visibility. KTP OPO: potassium titanyl phosphate optical parametric oscillator, PPKTP: periodically poled potassium titanyl phosphate.

\begin{tabular}{|c|c|c|c|c|c|c|}
\hline Reference & Configuration & Observable & Bw. $(\mathrm{MHz})$ & $\lambda$ & $B$ & $V_{\text {net }}$ \\
\hline [Kuklewicz et al. 2006] & KTP OPO & polar. & 22 & 795 & 0.7 & $77 \%$ \\
\hline [Halder et al. 2007] & $\mathrm{PPLN} / \mathrm{W}$ & time-bin & 1200 & 1560 & 446 & NA \\
\hline [Bao et al. 2008] & PPKTP OPO & polar. & 9.6 & 780 & 6 & $97 \%$ \\
\hline [Piro et al. 2009] & PPKTP & polar. & 22 & 854 & 3 & $98 \%$ \\
\hline [Pomarico et al. 2009] & PPLN/W OPO & time-bin & 117 & 1560 & 17 & $94 \%$ \\
\hline [Yan et al. 2011] & Rubidium atoms & polar. & 6 & 780 & 0.5 & $90 \%$ \\
\hline This thesis [Kaiser et al. 2013] & $\mathrm{PPLN} / \mathrm{W}$ & polar. & 540 & 1560 & 306 & $99 \%$ \\
\hline This thesis [Kaiser et al. 2013] & $\mathrm{PPLN} / \mathrm{W}$ & polar. & 25 & 1560 & 380 & $99 \%$ \\
\hline
\end{tabular}

\subsection{Conclusion and perspectives}

In conclusion, we have demonstrated remarkably versatile experimental outcomes towards producing polarization entangled photon pairs in the telecom C-band of wavelength (1530 $1565 \mathrm{~nm})$. As discussed earlier, the versatility refers to the fact that the photons produced by our source can be engineered, in the spectral domain, to be adapted to a broad variety of quantum applications. Depending on the application, by adapting the phase-matching condition and the filtering solution, the single photon wavelength can be tuned over more than $100 \mathrm{~nm}$ and associated spectral bandwidth chosen over more than five orders of magnitude. However, we can fairly say that the versatility also covers the aspect of the entangled state creation as well. By exploiting the advanced fiber-based entanglement preparator connected to a highly efficient type-0 photon pair generator, we are capable of preparing any arbitrary superposition of all the maximally polarization entangled Bell states. Our measured values of the normalized source brightness also stand among the highest ever reported for narrow band entangled photon pair generation. Furthermore, the correlation measurement outcome represents the violation of the Bell-CHSH inequality by at least 40 standard deviations for all three filters, i.e. $100 \mathrm{GHz}$ DWDM , $540 \mathrm{MHz}$ PSFBG, and $25 \mathrm{MHz}$ PSFBG.

As has been discussed before in Chapter 3, we have suggested some possible improve- 
ments that can help maximizing the true potential of our entanglement source (see 5.2.7), and similar suggestions also hold true this type-0 source. However, we could see a considerable improvements in the overall experimental rate if the generated photon pairs were separated in a deterministic manner. This is feasible but the price to pay would be on even more complex overall experimental apparatus than the current setup of Fig.5.1. 


\section{Part III}

\section{High-rate heralded single photon}

sources at telecom wavelength 

Chapter 6

\section{Prominent strategies for producing}

\section{single photons}

\section{Contents}

6.1 Single photon sources: state-of-the-art . . . . . . . . . . 125

6.1.1 True single photon generation . . . . . . . . . . . . . . 127

6.1.2 Non-linear optics based, heralded single photon sources . . . . . . . 129

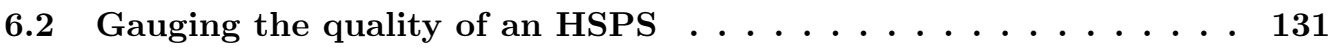

6.3 Battling the multi-photon $\ldots \ldots \ldots \ldots \ldots \ldots$

Photons play a pivotal role towards enabling various optical quantum technologies. Therefore, the development of high-quality photon sources that produce pairs of entangled photons, or simply a stream of well timed indistinguishable single photons, is required. Two demonstrations of polarization entangled photon pair sources for this thesis work have already been described in Chapters 4 and 5. Meanwhile, the next following Chapters 7 and 8 of this manuscript will deal, particularly, with the development of the so-called heralded single photon sources.

\subsection{Single photon sources: state-of-the-art}

Single photons remain one of the key ingredient for wide variety of quantum applications:

- In quantum key distribution (QKD), a qubit of information can be encoded, for instance, on the polarization state of a single photon. During the transfer of information, should more than one photon carry the same value of qubit at a given time window, 
an eavesdropper can exploit the photon number splitting (PNS) attack to extract part of the information [Brassard et al. 2000, Scarani et al. 2009]. Hence, single photon states are required to ensure the security of QKD.

- One of the emerging aspect of quantum information science is linear optical quantum computing (LOQC) [Knill et al. 2001]. With regard to LOQC, researchers manipulate single photon states by exploiting the very basic building blocks of linear optics such, as beam splitters, half-wave and quarter-wave plates, and phase shifters. More importantly, the so-called LOQC-based, CNOT gates which represent a critical "computing" element to LOQC [O'Brien et al. 2003, Nemoto \& Munro 2004], strictly require single photons so as to ensure the correct intended functionality. In parallel, single photons are also being used in boson sampling experiments [Tillmann et al. 2013, Broome et al. 2013].

- In quantum metrology, the calibration of, specifically, number-resolving single photon detectors should be done at the single photon level, hence the requirements of single photons [Polyakov \& Migdall 2009, Avella et al. 2011].

The above statements alone represent enough reasons to call for the development of highquality, single photon sources (SPS) for quantum information science (QIS) applications. Ideally, an SPS should be able to emit indistinguishable single photons in a deterministic way, i.e. on-demand, at an arbitrarily high repetition rate, and with zero probability of multi-photon emission [Scarani et al. 2009]. Ideal single photon sources should emit light in the Fock state $|1\rangle$. In experiments, the most common, yet trivial, means to attain the single photon regime is to strongly attenuate a laser beam so as to ensure negligible multi-photon generation events. In quantum physics, coherent states represent the most "classical" states of the harmonic oscillator, and they can be used to describe the quantum state of a laser. Basically, they are the eigenstates of the annihilation operator

$$
\hat{a}|\alpha\rangle=\alpha|\alpha\rangle
$$


and they can be expressed in the basis of number states, i.e. Fock states as:

$$
|\alpha\rangle=e^{\frac{-|\alpha|^{2}}{2}} \sum_{n} \frac{\alpha^{n}}{\sqrt{n !}}|n\rangle=e^{\frac{-|\alpha|^{2}}{2}}\left[|0\rangle+\alpha|1\rangle+\frac{\alpha^{2}}{\sqrt{2 !}}|2\rangle+\ldots\right] .
$$

The associated photon distribution follows Poissonian statistics, with a probability of obtaining $n$ photon(s) in a given time interval:

$$
P_{n}=e^{-\bar{n}} \frac{\bar{n}^{n}}{n !}
$$

where the average number of photons $\bar{n}=|\alpha|^{2}$ and the variance of $\Delta n^{2}=\overline{n^{2}}-\bar{n}^{2}=\bar{n}$. In most practical applications of quantum communication, the mean number of photons produced per pump pulse is kept below 0.1 [de Riedmatten et al. 2004b]. In this configuration, multi-photon events are negligible and $P_{1} \gg P_{n \geq 2}$. However, when imposing a low $\bar{n}$, one can straightforwardly deduce from Eq.(6.3) that vacuum represent the biggest chunk in photon distribution, i.e. $P_{0} \gg P_{1}$. Therefore, this type of source cannot be considered as an "ideal" single photon source.

\subsubsection{True single photon generation}

The quest for a true single photon source has continued with the exploitation of single quantum emitters [Grangier et al. 2004] such as quantum dots [Santori et al. 2001, Press et al. 2007], and color center in diamond [Brouri et al. 2000, Kurtsiefer et al. 2000]. A single photon source can be engineered from III-V semiconductor heterostructures. Particularly, quantum dots (QD) can be fabricated in galium arsenide(GaAs) where the use of, not just optical [Santori et al. 2001], but also electrical pumping is possible. The electrical pumping of such structure was first demonstrated in [Yuan et al. 2002] and previously considered as one of the major breakthroughs towards the realization of a robust and compact, future single photon source. In order to increase the emission rate and the directionality, researchers exploit the Purcell effect where a single quantum dot is deposited into a microcavity [Pelton et al. 2002, Bennett et al. 2005, Press et al. 2007, Wilk et al. 2007]. To date, both optically and electrically pumped sources have been proved to deliver indistinguishable, transform limited single photons [Santori et al. 2001, Bennett et al. 2008]. However, the investigation 
of, namely entanglement via the violation of Bell's inequality [Fattal et al. 2004b], as well as quantum teleportation [Fattal et al. 2004a], are demonstrated with optically pumped sources due to poor single photon indistinguishability from electrically driven ones. Apart from that, QDs have also been integrated in photonic crystal waveguides to produce single photons at telecom $\mathrm{O}$ band of wavelengths [Hoang et al. 2012]. A major drawback shared by all previously mentioned QD implementations is the requirement of cryogenic working condition due to limited material quality. Recently, a group of researchers in Japan has successfully demonstrated, for the first time, a single photon emission at room temperature by a gallium-nitride QD, fabricated at a pre-defined location [Holmes et al. 2014].

The nitrogen-vacancy (NV) center in diamond represents the first single color center ever detected [Gruber 1997] and since became the most widely studied approach towards producing single photons in solid-state. One of the biggest advantage of NV centers is the excellent stability at room temperature thanks to the rigidity of the diamond lattice. Furthermore, previously low luminescence collection efficiency due to high refractive index of the diamond itself has been solved by the use of nanocrystals, and recently, solid immersion lens technique [Marseglia et al. 2011] has become the preferred solution. There also has been a tremendous progress concerning the implementation of integrated diamond photonics. Recently, the coupling of individual NV centers to a microring resonator fabricated in singlecrystal diamond is demonstrated [Faraon et al. 2011]. Apart from that, hybridization aspect has also been demonstrated where a single NV center is successfully coupled to a fiberbased Fabry-Perot cavity in free-space [Albrecht et al. 2013]. Furthermore, an electrically driven single photon emission based on a single neutral NV center was demonstrated from a successful collaboration between the groups in Japan and Germany [Mizuochi et al. 2012]. Albeit the advantages presented so far, color centers in diamond, generally, only emits single photons in the visible range of wavelength, preventing such sources to be implemented in a long distance QKD links. Overall, NV centers might be a good choice for generating single photons for conventional scientific applications such as biological process imaging, but, the difficulty to obtain Fourier transform limited photons from NV centers by cryogenic cooling [Tamarat et al. 2006] made it less attractive for QIS applications. 


\subsubsection{Non-linear optics based, heralded single photon sources}

With regard to single quantum emitter approach, another fundamentally different strategy towards generating single photons is to implement heralded single photon source (HSPS) [Rarity et al. 1987]. Heralding technique is applicable when two simultaneously generated photons are available. Upon separation, one of the two photons is detected and this serves to announce the existence of the second one. In the meantime, the second photon, i.e. the heralded photon can propagate through a series of manipulation before being routed to the final destination. HSPS realizations have been demonstrated in asynchronous [Fasel et al. 2004, Alibart et al. 2005, Brida et al. 2012] and synchronous [Pomarico et al. 2012, Krapick et al. 2013], pair generation regimes. Through heralding, one can alter in a positive manner, the distribution of the heralded photons. As a result, a delivery of single photons approaching the ideal case of an SPS is obtained.

In experiments, pairs of photons are conveniently generated via:

- Four wave mixing $(\mathrm{FWM})$ in $\chi^{(3)}$ materials.

- Spontaneous parametric down conversion (SPDC) process in $\chi^{(2)}$ materials.

Due to probabilistic nature of of both nonlinear processes mentioned above, HSPS realization from ideal SPS as:

- HSPS is not an on-demand single source due to randomly generated photon pairs.

- HSPS is not a true single photon source due to multi-photon generation events, that cannot be discriminated due to the current technological limitation of photon number resolving detection capability concerning the heralding photons.

Despite this, an HSPS is indeed a viable alternative to single emitter technology towards producing single photons and at the same time it provides greater advantages over the use of weak coherent pulses.

Recently, FWM has experienced booming interests towards generating photon pairs in optical fibers [Fiorentino et al. 2002, Li et al. 2004, Takesue \& Inoue 2004, Dyer et al. 2009], photonic crystal fibers [Fan et al. 2005, Rarity et al. 2005, Fulconis et al. 2005] and more 
particularly in silicon nanophotonic devices [Sharping et al. 2006, Harada et al. 2008, Clemmen et al. 2009, Xiong et al. 2011]. In particular, with silicon nanophotonics, one can dream of future scalable quantum processing on-chip. Benefiting from the compatibility to CMOS technology platform and from the very high index of the silicon itself, dense integrated photonic chips can be fabricated [Bogaerts et al. 2006]. The efficiency of $\chi^{(3)}$ in silicon based devices can be significantly improved by exploiting the dispersion-engineered slow-light waveguide structure [Clark et al. 2013, Xiong et al. 2013]. For the first time, the produced heralded single photons at room temperature have been characterized in a $\chi^{(3)}$ silicon nanophotonic device [Davanço et al. 2012], and followed by [Clark et al. 2013]. More advanced multiplexing techniques so as to increase the performance of silicon-based HSPS are demonstrated [Collins et al. 2013,Zhang et al. 2015]. Additionally, the generation of photon pairs through FWM in silicon based devices has also led to the investigations of photonic entanglement generation, either outside [Takesue et al. 2007b, Wakabayashi et al. 2015], or on-chip configuration [Grassani et al. 2015, Xiong et al. 2015b].

Despite these promising results, however, the performance of FWM sources are heavily affected by Raman scattering and multi-photon absorption: as a matter of fact, these effects combined with generally small $\chi^{(3)}$ coefficients, result in limitations of the source brightness [Clark et al. 2012, Husko et al. 2013].

As valuable alternative to $\mathrm{FWM}, \mathrm{SPDC}$ in $\chi^{(2)}$ materials is today considered to be a main contributor to the continuous development of heralded single photon sources. As already seen in previous chapters, precise engineering of suitable $\chi^{(2)}$ materials via periodically poling can lead to an accurate phase matching condition where production of photon pairs at telecom wavelength can be realized [Tanzilli et al. 2002] as essential for long distance quantum transmission in optical fibers [Alibart et al. 2005, Aboussouan et al. 2010]. Furthermore, fine tuning of the phase matching can be easily achieved, either by modifying the pump wavelength or by adjusting the temperature of the material. Although, generated photon pairs via SPDC generally exhibit spectral correlations leading to a reduced purity of generated quantum states [Grice et al. 2001], such weakness can easily be compensated via suitable spectral filtering [Mosley et al. 2008a, Mosley et al. 2008b, Martin et al. 2009, Aboussouan et al. 2010]. Eventually, concerning the efficiency, SPDC is highly preferred to FWM as the second order non-linearity is much stronger than the third one. 
SPDC also has the reputation of being the most convenient tool for generating photonic entanglements, empowering various studies on quantum phenomena [Kwiat et al. 1995, Tanzilli et al. 2002, Kaiser et al. 2012a]. For these various reasons, SPDC $\chi^{(2)}$ remains the preferred photon pair generators towards generating photon pairs in HSPS implementation. Based on what has been discussed, we have opted for SPDC to be the workhorse for generating photon pairs in our HSPS realizations.

\subsection{Gauging the quality of an HSPS}

It is interesting to compare the performance of an HSPS to an ideal SPS. An ideal SPS should emit only single photons separated by a specific interval of time. By considering the previous statement, let us assume a thought experiment. For now, we consider a stream of well separated single photons as shown in Fig.6.1 (a). Quantumly speaking, if a single photon is incident on a 50/50 beam splitter, it will either be transmitted or reflected with an equal probability of $\frac{1}{2}$. Accordingly, if two single photon detectors $D_{1}$ and $D_{2}$ (with negligible dark counts) are placed separately at each output of the beam splitter, no temporal coincidences between their detection events will be observed. This is what is famously acknowledged as the antibunchness of a single photon.

(a)

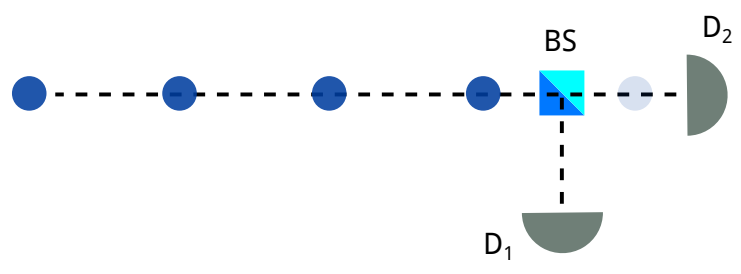

(b)

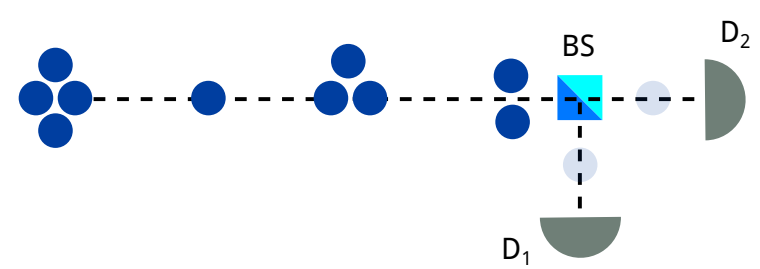

Figure 6.1: The thought experiment. (a): a stream of well separated single photons arriving at a beam splitter. (b): the single photons arrive in bunches at the beam splitter. 
In case of non negligible multi-photon contributions, we can imagine that bunches of simultaneous photons arrive at the beam splitter (see Fig.6.1 (b)). On average, half of the photons will be directed to $D_{1}$ and the other half, of course, will found the way to $D_{2}$ and coincidences between the detections of $D_{1}$ and $D_{2}$ can appear. A brilliant idea to observe the photon bunching was first established by Hanburry Brown and Twiss for an application in astrophysics [Hanbury Brown \& Twiss 1956]. In order to analyze the intensity correlations in a quantifiable manner, the second-order correlation function of light $g^{(2)}(0)$ is used and it is defined by

$$
g^{(2)}(\tau)=\frac{\langle I(t+\tau) I(t)\rangle}{\langle I(t)\rangle\langle I(t+\tau)\rangle}
$$

Here, $I(t)$ represents the intensity of the light beam at time $(t)$.

Eq.(6.4) can be rewritten as

$$
g^{(2)}(\tau)=\frac{\left\langle N_{D_{2}}(t+\tau) N_{D_{1}}(t)\right\rangle}{\left\langle N_{D_{1}}(t)\right\rangle\left\langle N_{D_{2}}(t+\tau)\right\rangle}
$$

since the recorded rates $N_{D_{1}}(t)$ and $N_{D_{2}}(t)$ for both detectors $D_{1}$ and $D_{2}$, respectively, are proportional to the intensity. Here, the second-order correlation function $g^{(2)}(\tau)$ signifies the correlation of detected photons at $D_{1}$ and $D_{2}$ separated by a time $\tau$. For bunched light, $g^{(2)}(0)>1$ where $1 \leq g^{(2)}(\tau)<g^{(2)}(0)$. Meanwhile, for the antibunched light $g^{(2)}(0)<1$ and in the case of an ideal SPS $g^{(2)}(0)=0$ (pure quantum effect described at the beginning of the thought experiment). Aside from that, coherent light exhibits $g^{(2)}(\tau)=1$ for all $\tau$.

A basic experimental arrangement for measuring $g^{(2)}(\tau)$ of an HSPS is depicted in Fig.6.2. The SPDC-based source is employed for creating photon pairs comprising signal and idler photons. Upon successful detection of a (heralding) signal photon by the detector $D_{H}$, trigger signals are released so as to gate the opening of detectors $D_{1}$ and $D_{2}$ placed in the HBT detection system.

For an HSPS, we are particularly interested in the second-order correlation $g^{(2)}(\tau)$ at zero delay. Accordingly, we are going to express $g^{(2)}(0)$ in term of photon number probabilities in weak gain regime $\left(P_{2} \gg P_{n \geq 3}\right)$. We will consider the case of negligible dark counts which is consistent to our experiments. We begin with the probability, $P_{D_{1}}^{(1)}$, to have a click in 


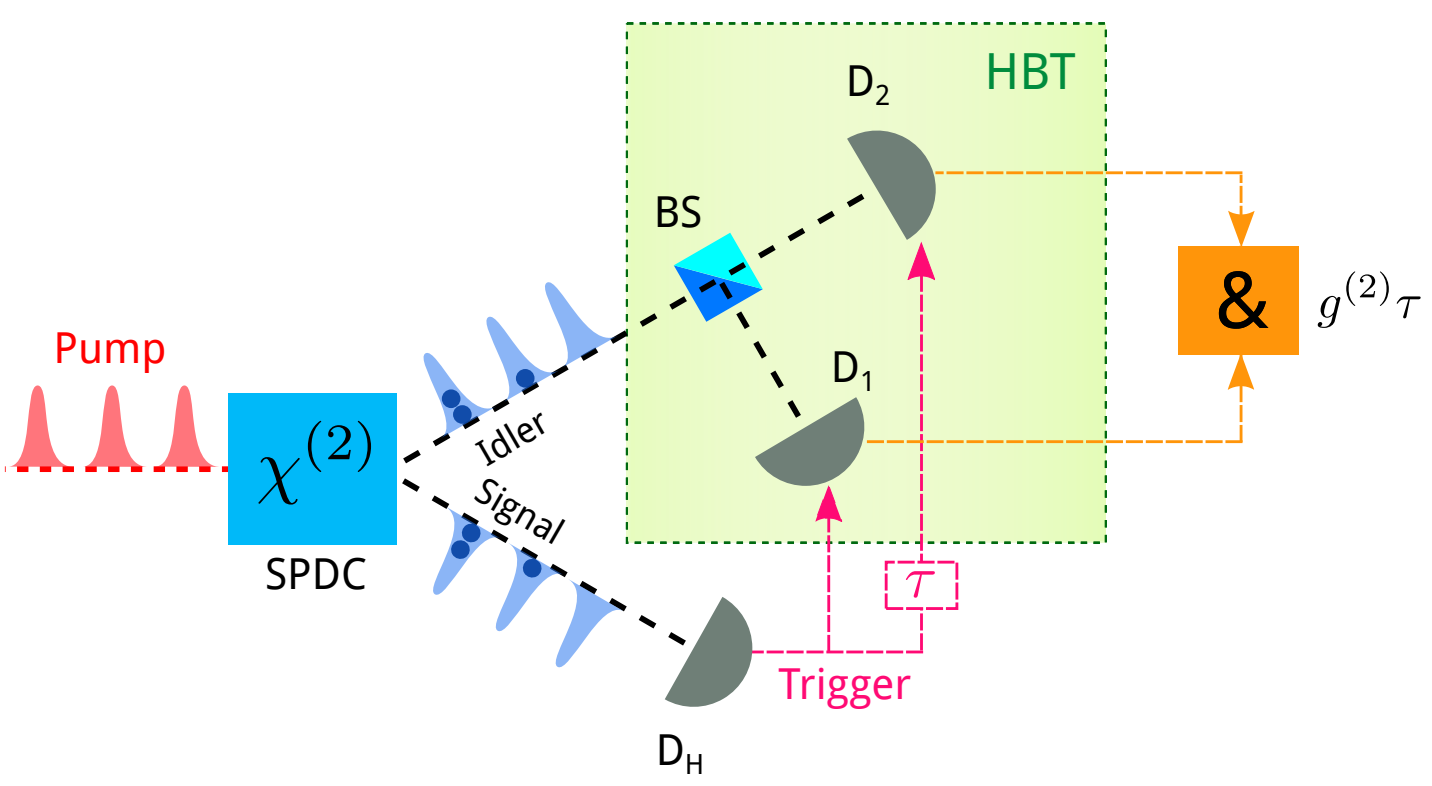

Figure 6.2: Original example behind the implementation of heralded single photon source, extended to a Hanburry Brown and Twiss (HBT) type configuration for measuring secondorder correlation $g^{(2)}(\tau)$. Nonlinear SPDC interaction in $\chi^{(2)}$ material is exploited for photon pair generation. Upon successful detection of signal photons by detector $D_{s}$, an electrical signal will be released and it will be used to trigger the opening of two other installed detectors, $D_{i 1}$ and $D_{i 2}$, in the HBT section. Eventually, the coincidence rates between $D_{i 1}$ and $D_{i 2}$ are recorded by an AND gate (\&). BS: beam splitter. 
detector $D_{1}$ given only one heralded photon is available in front of the beam splitter,

$$
P_{D_{1}}^{(1)}=\frac{\eta_{D_{i 1}} \cdot P_{1}}{2}
$$

where $\eta_{D_{1}}$ denotes the detection efficiency of detector $D_{1}$ and $P_{1}$ is the probability of having only one heralded photon per heralding event. Additionally, the factor $\frac{1}{2}$ comes from the beam splitter whereby the photon is transmitted or reflected with $\frac{1}{2}$ probability. Similarly, the probability to have a click in detector $D_{2}, P_{D_{2}}^{(1)}$, can be described by

$$
P_{D_{2}}^{(1)}=\frac{\eta_{D_{2}} \cdot P_{1}}{2}
$$

In the cases where two photons are available just before the HBT setup, the probability to have a simultaneous click event in both $D_{1}$ and $D_{2}$ can be written as

$$
P_{D_{1} D_{2}}^{(2)}=\frac{\eta_{D_{1}} \cdot \eta_{D_{2}} \cdot P_{2}}{2}
$$

Here, $P_{2}$ denotes the probability to have two simultaneous heralded photons impinging on the beam splitter. As for the main figure-of-merit, the second-order correlation function of $g^{(2)}(0)$, which quantify the singleness of the generated heralded photons can simply be expressed as

$$
g^{(2)}(0)=\frac{P_{D_{1} D_{2}}^{(2)}}{P_{D_{1}}^{(1)} \cdot P_{D_{2}}^{(1)}} .
$$

By replacing all the parameters in Eq.(6.9) with previously obtained in Eqs.(6.6), (6.7) and (6.8), we have

$$
g^{(2)}(0)=\frac{2 P_{2}}{P_{1}^{2}}
$$

Alternatively, the performance of an HSPS can also be discussed in term of the coincidence-to-accidental ratio $(C A R)$ [Harada et al. 2010]. Experimentally speaking, coincidence event registered between, for instance, $D_{H}$ and $D_{1}$ (see Fig.6.2) can be processed by a coincidence counter. The outcomes from such a device can be visualized in term of coincidence histogram. From the example shown in Fig.6.3, we can see a series of coincidence peaks separated by the repetition rate of the employed laser. In this case, the ratio between 
the counts in the highest and one of the small peaks defines the $C A R$. True coincidence events caused by the paired photons generated by the same pump pulse can only be found in the highest peak at zero delay. However, accidental coincidence counts due to multi-photon events by the same pump pulse also contribute to this peak. Meanwhile, the other small lateral peaks contain mostly accidental counts due to dark counts of the employed detectors $d_{D_{H}, D_{1}}$, and photon pairs generated by neighboring pump pulses.

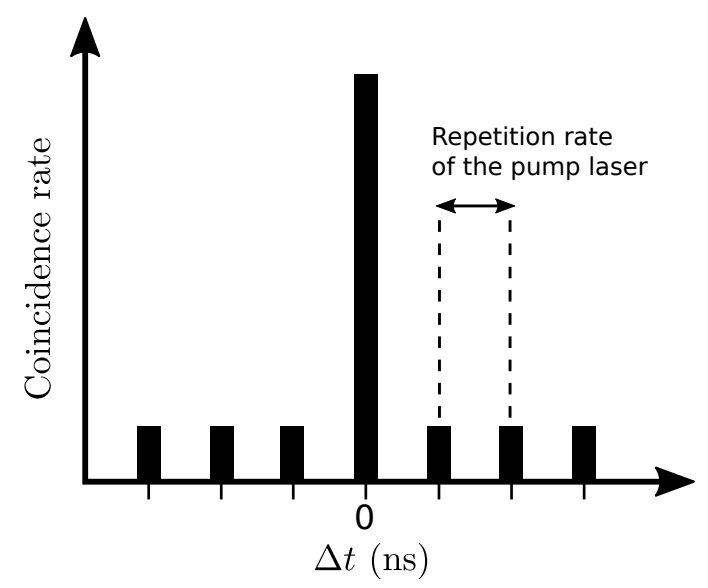

Figure 6.3: An example of coincidence histogram acquired by a coincidence counter. Each peak is separated by the repetition rate of the employed pump laser.

In order to calculate the $C A R$, we first define the coincidence rate between $D_{H}$ and $D_{1}$ :

$$
R_{C}=f \cdot \bar{n} \cdot \mu_{s} \mu_{i}
$$

where $f$ is the laser repetition rate, $\bar{n}$ denotes the mean number of photon pairs produced per pump pulse, and $\mu_{s, i}$ represents the overall single photon transmission in both signal $(s)$ and idler $(i)$ arms comprising coupling, i.e. collection, propagation and quantum detection efficiencies. Meanwhile, the accidental count rate is given by

$$
R_{C-A}=f\left[\left(\bar{n}+\bar{n}_{s}\right) \mu_{s}+d_{D_{H}}\right]\left[\left(\bar{n}+\bar{n}_{i}\right) \mu_{i}+d_{D_{1}}\right],
$$

where $\bar{n}_{s, i}$ is the probability of having a noise photon per pump pulse. 
Therefore, $C A R$ can be described as

$$
C A R=\frac{R_{C}+R_{C-A}}{R_{C-A}}=\frac{\bar{n} \mu_{s} \mu_{i}}{\left[\left(\bar{n}+\bar{n}_{s}\right) \mu_{s}+d_{D_{H}}\right]\left[\left(\bar{n}+\bar{n}_{i}\right) \mu_{i}+d_{D_{1}}\right]} .
$$

From Eq.(6.13), we can see that in weak and high gain regimes, the noise affecting the $C A R$ mainly comes from the dark count of the employed detectors and the multi-photon generation, respectively.

Both the second-order correlation function of $g^{(2)}(0)$ and the $C A R$ can be used as the figures-of-merit so as to gauge the performance of an heralded single photon source. In various HSPS demonstrations, the $g^{(2)}(0)$ represents the most highlighted characteristic which quantify the singleness of the heralded photons. The $C A R$ on the other hand is useful, for instance, in estimating the error rate of an entanglement-based quantum key distribution implementation [Takesue \& Inoue 2005].

\subsection{Battling the multi-photon}

Aside from having better $g^{(2)}(0)$ and $C A R$, among others, an HSPS should deliver single photons at an arbitrarily high rate. A simple gesture of increasing the pump power remains the easiest answer to such demand. This simply translates into higher mean number of photon pairs produced per pump pulse $\bar{n}$. Despite the convenience, increasing the pump power is not the best solution as the source is polluted more and more by multi-photon generation. New techniques should be introduced in order to increase the performance of the source in term of higher single photon rates while keeping the level of $\bar{n}$ to the minimum. Among the earliest, spatial multiplexing of separated photon pair sources has been proposed [Migdall et al. 2002]. The simplistic idea behind this scheme is to simultaneously generate photon pairs in separated sources but at low probability. Even though single photon rates per source is not high enough, collectively, the rates are greater. The major downside of this scheme is the scability, and this will become the aim of the next Chapter 7 .

Another technique is to implement passive time multiplexing in pulse regime. In a sense, this looks like much easier to be realized compared to the complicated spatial multiplexing scheme. The basic idea is to compensate the reduction of the pump power by increasing 
the repetition rate of the pump laser. Unlike previous approach, this technique somehow shows a very strong technological dependency. Nevertheless, the realization of an ultra-fast HSPS is dedicated in Chapter 8.

Meanwhile, very recently, researchers are also interested in demonstrating active temporal multiplexing in order to increase single photon generation probability [Mendoza et al. 2015, Xiong et al. 2015a, Kaneda et al. 2015] where photon pair generation are repeated continuously and through a network of optical switches, the photons are sent to a single mode output. 



\section{Spatially multiplexed single photons}

\section{through hybridization}

\section{Contents}

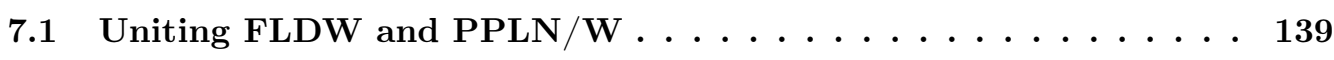

7.1.1 Characterization of the PPLN waveguides . . . . . . . . . 141

$7.1 .2 \quad$ Integrated FLDW chips $\ldots \ldots \ldots \ldots \ldots \ldots$

7.2 Experimental arrangement highlighting hybridization $\ldots \ldots \ldots 144$

7.2.1 Active and fast switching strategy . . . . . . . . . . . . 145

7.3 Experimental results and discussion $\ldots \ldots \ldots \ldots \ldots$

7.4 Conclusion and perspectives of the source $\ldots \ldots \ldots \ldots$

Some results presented in this chapter were published in T. Meany, L. A. Ngah et al., Hybrid photonic circuit for multiplexed heralded single photons, Laser \& Photonics Reviews, Volume 8, Issue 3, pages L42-L46, May 2014.

\subsection{Uniting FLDW and PPLN/W}

As has been discussed earlier in Chapter 6, one way to improve the performance of an heralded single photon source is by spatially multiplexing several SPDC sources. Ideally, this scheme allows obtaining greater brightness, while maintaining a certain accepted level of the injected pump power. Consequently, this leads to the lessening of multi-photon generation events and to the reduction of overall noise level. However, the spatial multiplexing strategy contributes to the size augmentation of such devices and moreover, the stability will become even more critical aspect affecting its performances. 
Today, integrating several waveguides in the same non-linear crystal, e.g. periodically poled lithium niobate (PPLN) crystal represents a standard achievement. For example, the homemade PPLN sample employed in our experiment contains more than hundred periodically poled waveguides. At the same time, femtosecond laser direct-write (FLDW) chips allow to simultaneously send and collect photons from different PPLN/Ws by buttcoupling technique. By butt-coupling, we mean that light exiting the PPLN/W can be coupled directly into the FLDW chip without any intermediary. Since first demonstrated in 1996, FLDW continue to be one of the most emerging research branch in integrated photonics [Davis et al. 1996]. The fabrication of FLDW chips resorts to the use of short, femtosecond-pulse laser which is tightly focused in high-quality transparent materials. The focused laser beam then induces a permanent and localized refractive index in the exposed area through nonlinear absorption [Little et al. 2008, Gross et al. 2012]. Thanks to the incredibly high-precision of modern control stages used to translate the movement of the transparent material with respect to the focalized laser beam, waveguide structures can easily be formed according to the planned design. Moreover, these waveguides can be formed at multiple depth of the transparent material, allowing the fabrication of the socalled 3-dimensional integrated chips [Owens et al. 2011, Crespi et al. 2013]. Additionally, evanescent-wave coupling can be achieved when two waveguides are inscribed close to each other making it possible to produce directional couplers-based devices such as wavelength division multiplexing (WDM) filters which are to be used later in our experiment.

By combining these two different technologies, we achieve the size reduction, i.e. miniaturization of the experimental setup, thus considerably increasing the overall stability of the system. In this chapter, we want to highlight, in particular, that such a new hybridization has never been demonstrated before. Through this hybridization, we want to demonstrate a spatially multiplexed heralded single photon source. The performances of the source are evaluated in terms of the coincidence-to-accidental ratio $(C A R)$. This hybridization can be achieved with ease thanks to the following factors:

- FLDW technique allows fabrication of waveguides whose the mode size is relatively close to that of PPLN waveguides and standard single mode fibers, hence justifying the implementation of butt-coupling method. 
- The high-quality glass material shows relatively large transparency range of wavelengths, including visible and infrared ranges, which are at the center of interest in quantum information science. This statement proves the use of two FLDW integrated chips to send and to collect, before and after the PPLN/Ws, both visible and infrared photons.

\subsubsection{Characterization of the PPLN waveguides}

In our experiment, a home-made PPLN sample is employed comprising a simultaneous use of four different waveguides. Through SPDC, for a pump wavelength at $710 \mathrm{~nm}$, the four waveguides each generates signal and idler photons in telecom wavelengths of $1310 \mathrm{~nm}$ and $1550 \mathrm{~nm}$, respectively. At the given pump wavelength, the main key is to have four waveguides exhibiting, more or less, the same phase matching condition for a fixed temperature stabilization. This represents a crucial requirement for the spatially multiplexed heralded single photon source. Simple enough, this can be identified by observing the SPDC emission spectra of the corresponding waveguides.

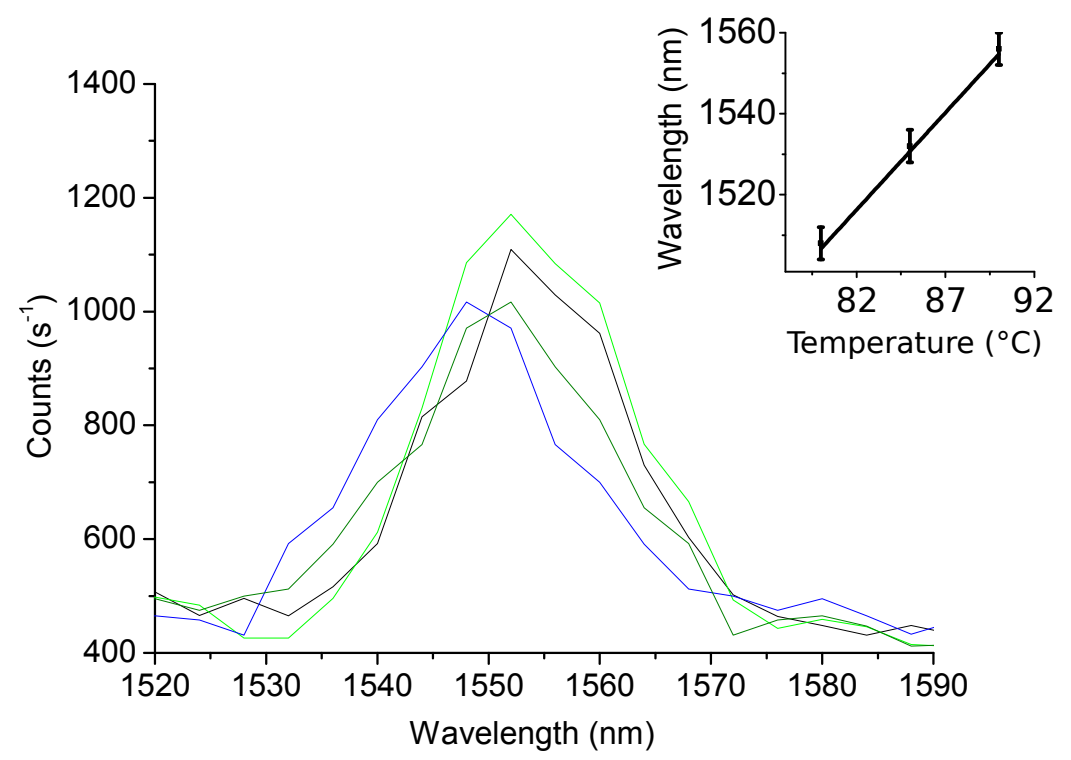

Figure 7.1: Output spectra for individual SPDC waveguides are color coded: PPLN 1 (black), PPLN 2 (light green), PPLN 3 (dark green), and PPLN 4 (blue). Due to the engineering of the PPLN/Ws, the spectra width of the idler photons are measured to be $30 \mathrm{~nm}$, centered around the wavelength of $1550 \mathrm{~nm}$. The inset describes the temperature dependence of the central wavelength. 
A selection of four waveguides had been characterized and good overlap is observed from multiple spectrum as can be seen in Fig.7.1. Here, the individual SPDC emission spectra are color coded: PPLN $1 \rightarrow$ black, PPLN $2 \rightarrow$ light green, PPLN $3 \rightarrow$ dark green, and PPLN $4 \rightarrow$ blue. Due to the engineering of the PPLN waveguides, the spectra width of the idler photons around $1550 \mathrm{~nm}$ are measured to be $30 \mathrm{~nm}$. Such a phase matching condition is obtained through the use of four temperature stabilized $1 \mathrm{~cm}$ long waveguides at $90^{\circ} \mathrm{C}$, having the same width of $7 \mu \mathrm{m}$ and the same poling period of $13.8 \mu \mathrm{m}$. The pump wavelength for such measurement is recorded at $710.21 \mathrm{~nm}$. Furthermore, the emission spectrum can be comfortably tuned by changing the temperature of the PPLN sample and due to the limitation concerning the wavelength range of our tunable filter (Yenista XTM-50) used for the spectrum measurement, we only manage to obtain the spectrum centered around $1550 \mathrm{~nm}$. We note that the central wavelength of the $1550 \mathrm{~nm}$ spectrum can be shifted by $4 \mathrm{~nm}$ for each $1^{\circ} \mathrm{C}$ of temperature change (see inset in Fig.7.1).

\subsubsection{Integrated FLDW chips}

Two FLDW integrated photonic chips are fabricated uniquely for our experiment. The first one contains several $1 \rightarrow 4$ waveguide splitters, while the second one contains integrated, inline, wavelength division multiplexing (WDM) filters. The spacing between the waveguides on the edges of both FLDW chips is identical to the one between the waveguides in PPLN sample $\sim 190 \mu \mathrm{m}$, and the width of waveguides inscribed on both FLDW chips is $8 \mu \mathrm{m}$ so as to increase spatial mode matching and coupling efficiency across the three devices.

Basically, the $1 \rightarrow 4$ waveguide splitter is a four-way beamsplitter (BS) comprising four different inputs and four different outputs. One of the input on the front facet is pigtailed with a polarization maintaining fiber (PMF). During the design process, 3-dimentional interaction geometry technique was used in order to produce an equal splitting ratio across the outputs [Meany et al. 2012]. At first, an array of four waveguides are buried with an equal $7 \mu \mathrm{m}$ separation from each other. This initial array is then spread out resulting in a new separation of $190 \mu$ (see Fig.7.2). From the initial point, the $1 \rightarrow 4$ waveguide splitter is $15 \mathrm{~mm}$ in length.

Meanwhile, the second $3 \mathrm{~cm}$ long FLDW chip consists of four WDM filters. These identical, rather coarse filters are used for light separation based on the input wavelength. In our 


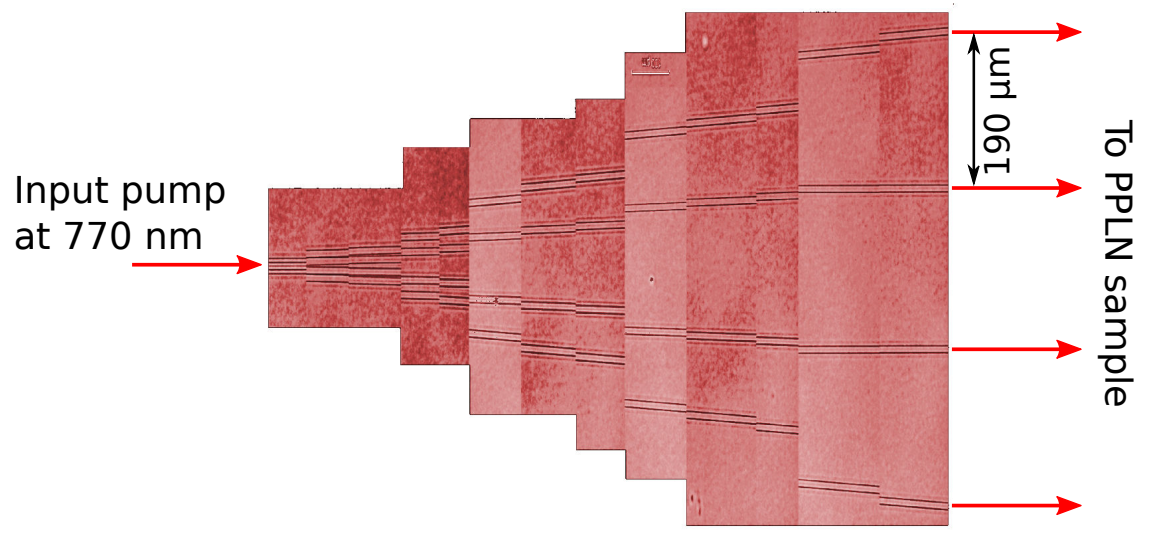

Figure 7.2: Stitched images of $1 \rightarrow 4$ waveguide splitter captured under a microscope. The pump light at around $710 \mathrm{~nm}$ is injected to one of the four pigtailed inputs. The light is then splitted and directed to the four outputs with equal intensity. The output waveguides are separated by $190 \mu \mathrm{m}$

case, attaining such a goal requires an accurate choice of device geometries, e.g. interaction region lengths, waveguide width and spacing, such that photons at different wavelength are directed towards different outputs with $100 \%$ transmission. For our experiment, the WDM filters are configured to separate lights between $1310 \mathrm{~nm}$ and $1550 \mathrm{~nm}$, with each wavelength region corresponds to a coarse transmission bandwidth of more than $20 \mathrm{~nm}$. Along the interaction region, the waveguide spacing is $7 \mu \mathrm{m}$. For the whole chip, all waveguides are $8 \mu \mathrm{m}$ in width. The wavelength dependent splitting ratio as a function of the coupling length at the $1310 \mathrm{~nm}$ output is illustrated in Fig.7.3. At an interaction length of $3.35 \mathrm{~mm}$, an extinction ratio of $10 \mathrm{~dB}$ is obtained where $\sim 90 \%$ is observed for $1310 \mathrm{~nm}$ light and $10 \%$ for the $1550 \mathrm{~nm}$ light. The recorded extinction ratio of $10 \mathrm{~dB}$ is comparable to previous reported demonstration [Eaton et al. 2009]. 


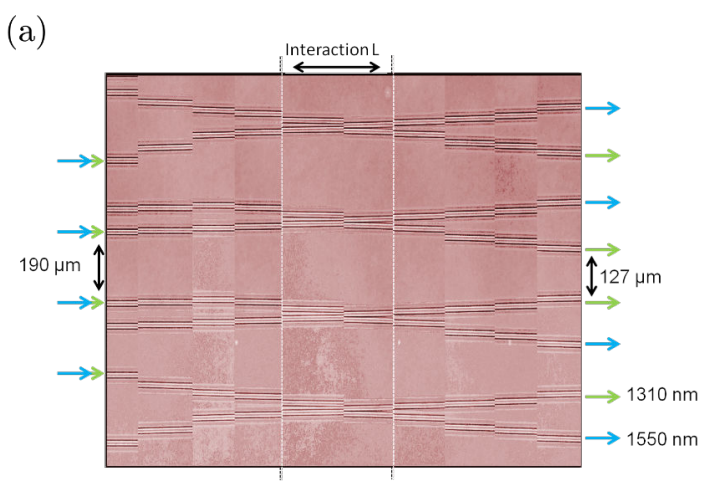

(b)

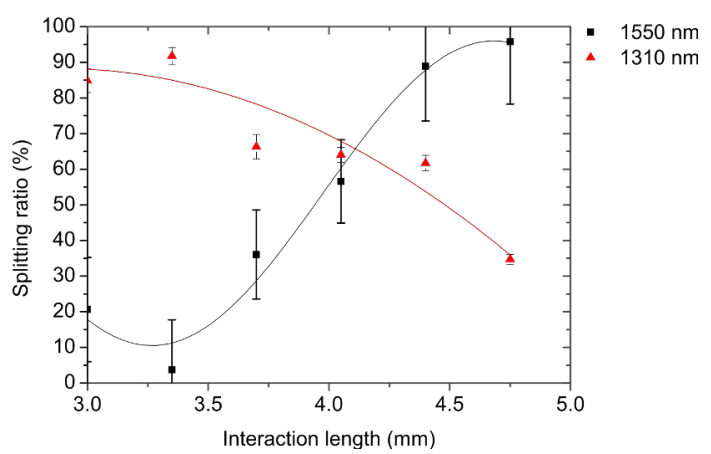

Figure 7.3: (a): The coarse WDM FLDW chip viewed under a microscope (reconstruction from stitched images) where the interaction regions are clearly visible. Four waveguides on the input facet are separated by $190 \mu \mathrm{m}$ which corresponds to the separation of waveguides in the PPLN chip. These inputs receive the down converted light consisting of signal (green arrow) and idler (blue arrow) photons from respective PPLN waveguides, and separate, spectrally, $1310 \mathrm{~nm}$ and $1550 \mathrm{~nm}$ lights from the full emission spectrum of the SPDC. (b): The splitting ratio as a function of the interaction region length is illustrated.

\subsection{Experimental arrangement highlighting hybridization}

The experimental setup of the spatially multiplexed heralded single photon source is illustrated in Fig.7.4. A pulsed, femtosecond laser (Coherent Chameleon) is employed as the pump laser. This particular laser originally provides $710 \mathrm{~nm}, \sim 100 \mathrm{fs}$ duration optical pulses at $76 \mathrm{MHz}$ repetition rate. The pump light is then directed towards a combination of diffraction gratings and standard spatial filter so as to provide linear pulse compression, resulting in broader pump pulse duration of $1.2 \mathrm{ps}$. This corresponds to an emission bandwidth of $\Delta \lambda_{p}=0.5 \mathrm{~nm}$. The pump light is then incident on a bulk half-wave plate (HWP) where the polarization of the light can be oriented at will. Next, through a lens, the pump light is coupled into a polarization maintaining fiber (PMF) which is pigtailed to the input of FLDW $1 \rightarrow 4$ waveguide splitter chip. The four outputs of this $1 \rightarrow 4$ splitter are aligned to four SPDC waveguides on a single type-0 PPLN sample, where signal and idler photons are generated via SPDC. Next to the PPLN chip, the FLDW chip integrating four $1310 \mathrm{~nm} / 1550 \mathrm{~nm}$ WDM filters is put in place, completing the hybridization of the system. In our experiment, the signal photons at $1310 \mathrm{~nm}$ serve as the heralding photons, announcing the existence of the heralded idler photons at $1550 \mathrm{~nm}$ in the setup. Here we stress that our source produces photon pairs at telecom wavelength thus allowing long distance 
propagation in standard optical fibers.

The heralding signal photons propagate in the so-called heralding arms, where they are eventually routed to a series of combined optical circulator and a core scanned, laser inscribed fibre Bragg gratings (FBG) filter [Williams et al. 2013] such that the reflected photons exhibit a spectral bandwidth of $85 \mathrm{GHz}$ at the central wavelength of $1312 \mathrm{~nm}$. These heralded photons are detected by four indium gallium arsenide (InGaAs) avalanche photo-diodes (APD, IDQ 210, 5 ns detection window, and $3 \mu$ s dead time) which are externally triggered by the repetition rate of the Chameleon laser at $76 \mathrm{MHz}$. Meanwhile in the heralded arms, the heralded idler photons pass through polarization controllers (PC) and adequate length of optical delays (optical fibers + electronically controlled optical variable delay) before arriving to a combination of high-speed, ultra-low loss lead lanthanum zirconum titanate (PLZT)-based, $2 \times 2$ optical switches (BATi Nanona ${ }^{\mathrm{TM}}$ ). The use of these optical switches is discussed in Sec.7.2.1. The PCs and the variable optical delays are used to ensure the polarization and temporal indistinguishability of all the produced heralded photons. Additionally, a tunable band-pass $100 \mathrm{GHz}$ filter (DiCon MTF-08) is placed at the switch combination output in order pick the heralded photons at $1548 \mathrm{~nm}$ who are energymatched with the heralding ones. The heralded photons are then detected by another APD (IDQ 201). It is important to note that, for both heralding and heralded photons, suitable spectral filtering are applied so as to ensure the purity in the single temporal mode of the heralded photons [Mosley et al. 2008b, Mosley et al. 2008a]. This is achieved through narrow filtering of the photons, leading to a coherence time greater than the pump pulse duration. Finally, a time interval analyzer (TIA, SensL HRM-TDC) is employed to measure timing delay between signals produced by both heralding and heralded detectors. The information processed by this electronic device is essential in evaluating the coincidences (both true or accidental ones, see Fig.7.5 for a conceptual example) thus leading to $C A R$ calculation, which is the figure-of-merit of our spatially multiplexed, heralded single photon source.

\subsubsection{Active and fast switching strategy}

In this experiment, the principal goal of the employed, active and fast switching strategy is to correctly route the heralded photon to the common output. Heralded photons available at the common output should be the ones that are correlated with detected heralding photons. 


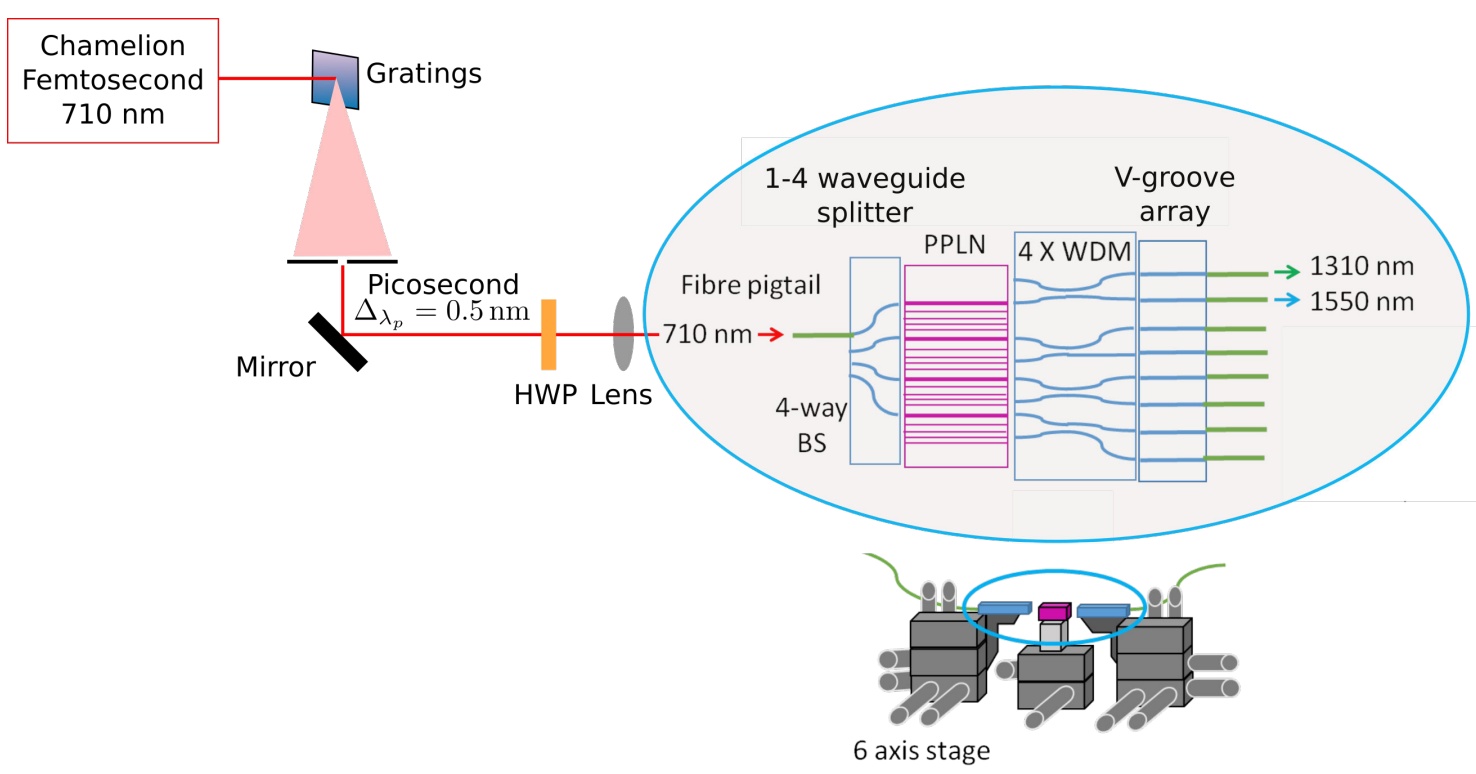

(a)

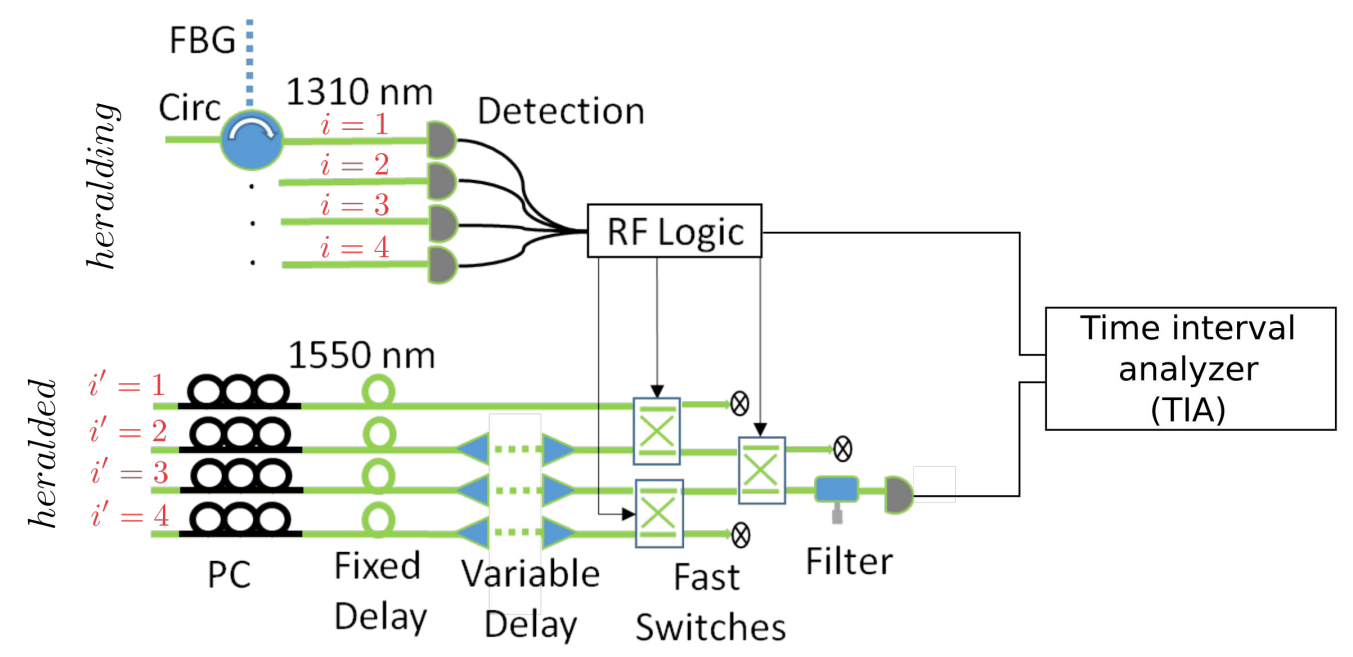

(b)

Figure 7.4: Experimental arrangement for the spatially multiplexed heralded single photon source. (a): Visualization of pump preparation stage and the hybrid system of integrated photonic chips. (b): Continuity from (a). Three fast photonic switches are controlled by a dedicated RF electronic circuit once the heralded photons are succesfully detected. A time interval analyzer (TIA) is used to register coincidences between the heralding and the heralded detectors which leads to coincidence-to-accidental ratio $(C A R)$ measurement. 


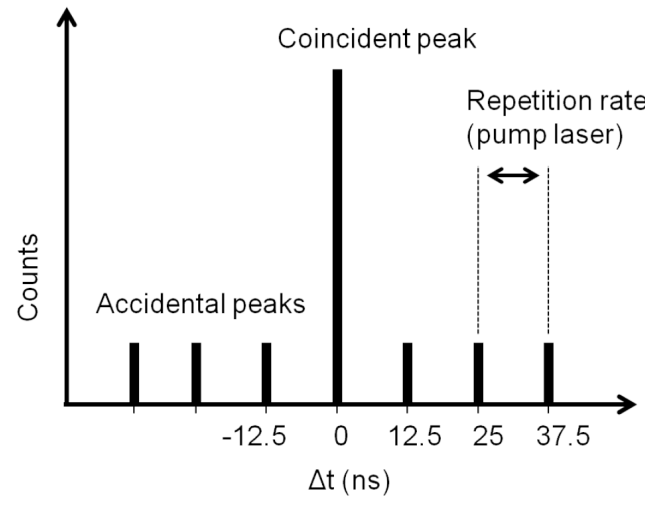

Figure 7.5: A conceptual example of coincidence histogram. Each peak is separated by $12.5 \mathrm{~ns}$, which corresponds well to the repetition rate of the laser.

As has been previously mentioned, these $2 \times 2$ optical switches are carefully aligned so as to implement an equivalent of a single $4 \times 1$ optical switch.

This can be achieved by implementing the configuration illustrated in Fig.7.4 (b). Upon successful detection of a heralding signal photon " $i$ " $(i=1,2,3,4)$, an electronic signal is sent to the switches on the heralded photon path so as to let only the corresponding heralded idler photon " $i$ " goes towards the detector. By doing so, the routing of paired photons can be guaranteed. In order to ensure the correct functionality of the employed switching strategy, the heralded photons need to be adequately delayed optically, prior their arrivals at these fast switches so as to allow relatively long, combined processing delays of the heralding detectors and the RF circuitry.

\subsection{Experimental results and discussion}

Some preliminary steps are taken before the experiment to ensure better overall results. As a matter of fact, experimental losses play a major role towards the reduction in coincidence rates, hence lower $C A R$. Therefore, every component is carefully selected from our inventory so as to reduce the overall insertion losses, thus leading to the increase of coincidence rate. The overall loss for each channel is measured (see Tab.7.1). We also employ optical filters with good extinction ratio in order to keep the photonic noise of the setup to the minimum.

First of all, the switches positions are fixed and we characterize individual PPLN waveguide by looking at the coincidence rate between the generated signal " $i$ " and idler " $i$ " pho- 


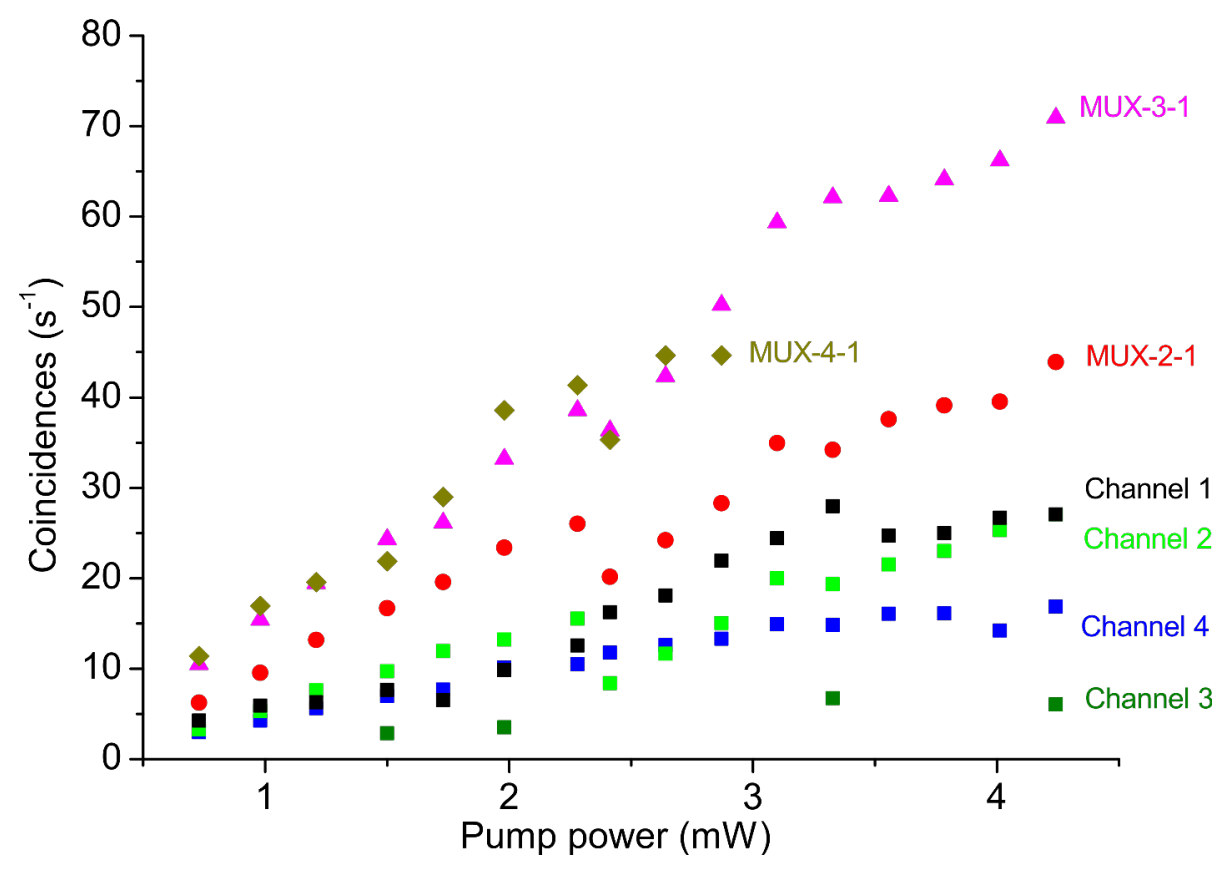

Figure 7.6: Characterization of both individual and multiplex PPLN configurations by looking at the coincidence rate as a function of the injected pump power. The measurement results are color coded: PPLN 1 (black), PPLN 2 (bright green), PPLN 3 (blue), PPLN 4 (dark green), PPLN 1 and 4 (MUX-2-1, red), PPLN 1, 2 and 4 (MUX-3-1, magenta), PPLN 1, 2, 3 and 4 (MUX-4-1, olive green). 
tons. Different measurement results are plotted as a function of the injected pump power in Fig.7.6. For individual waveguides, both PPLN 1 (black) and PPLN 2 (bright green) yields the highest coincidence rates of $27 \mathrm{~Hz}$ at a pump power of $4.25 \mathrm{~mW}$, followed by PPLN 4 (dark green) at $17 \mathrm{~Hz}$ and PPLN 3 (blue) at $6 \mathrm{~Hz}$. This variation is observed despite the overlap of SPDC emission spectrum from all PPLN waveguides. Notably, PPLN 3 shows the lowest obtained coincidences and this might due to multiple of reasons:

- The lack of inhomogeneity in term of the waveguide diameters.

- Possible damages in certain area on the input and output facets of the PPLN chip, affecting the waveguide in question.

- Low total internal SPDC efficiency inherent to the waveguide.

- Low efficiency of the heralding detector specifically used for channel 3.

Another important element that contributes to the variation of concidence rate across all PPLN channels is the alignment interfacing the PPLN and FLDW chips. Due to poor efficiency of PPLN 3, the alignment is done in favor of enhancing the coincidence rate of this specific waveguide.

Next, active multiplexing is applied thanks to the combination of fast optical switches. The combined PPLNs include PPLN 1 and 4 (MUX-2-1, red), PPLN 1, 2 and 4 (MUX-3-1, magenta) and finally PPLN 1, 2, 3 and 4 (MUX-4-1, olive green). As expected, compared to the result of individual PPLN, these multiplexed ones exhibit greater coincidence rate for every point of the experiment. However, by multiplexing all PPLNs (MUX-4-1), we observe, approximately, the same coincidence rates as the MUX-3-1 configuration. This is due to the lower overall efficiency of PPLN 3, as has been previously mentioned above. Another compelling observation from the recorded data is the apparent deviation from the linear trend of the measurement for the input pump power ranging from $2.2 \mathrm{~mW}$ to $3.2 \mathrm{~mW}$. At such higher pump power, the adhesive responsible for the pigtailing the $1 \rightarrow 4$ waveguide splitter to polarization maintaining fiber can expand with the temperature, thus reducing the coupling efficiency. Additionally, the damage threshold for such adhesive is at $20 \mathrm{~mW}$ of injected pump power, translating to a maximum of $4.5 \mathrm{~mW}$ being able to be injected to each PPLN. 
Fig.7.7 shows the measured $C A R$ for every individual PPLN as a function of the coincidences. Unsurprisingly, PPLN 3 has again exhibited the lowest $C A R$. Meanwhile, the measured $C A R$ for multiplex configurations are plotted in Fig.7.8. As expected, the improvement of the $C A R$ is proportional to the number of PPLN or sources being multiplexed, except for the configuration MUX-4-1. One way to interpret these results is by looking at a certain value of $C A R$. For example, at a $C A R$ range between 10 to 15 , the coincidence rate concerning the multiplex configurations is greater than the single PPLN one. This is equivalent to the fact that, by multiplexing, we manage to improve the generation of heralded photons while maintaining a constant level of multi-pair contributions. We also note that, for lower coincidence rate, the $C A R$ values are approximately the same as the heralding detectors exhibit relatively high dark count rates $>1 \mathrm{KHz}$.

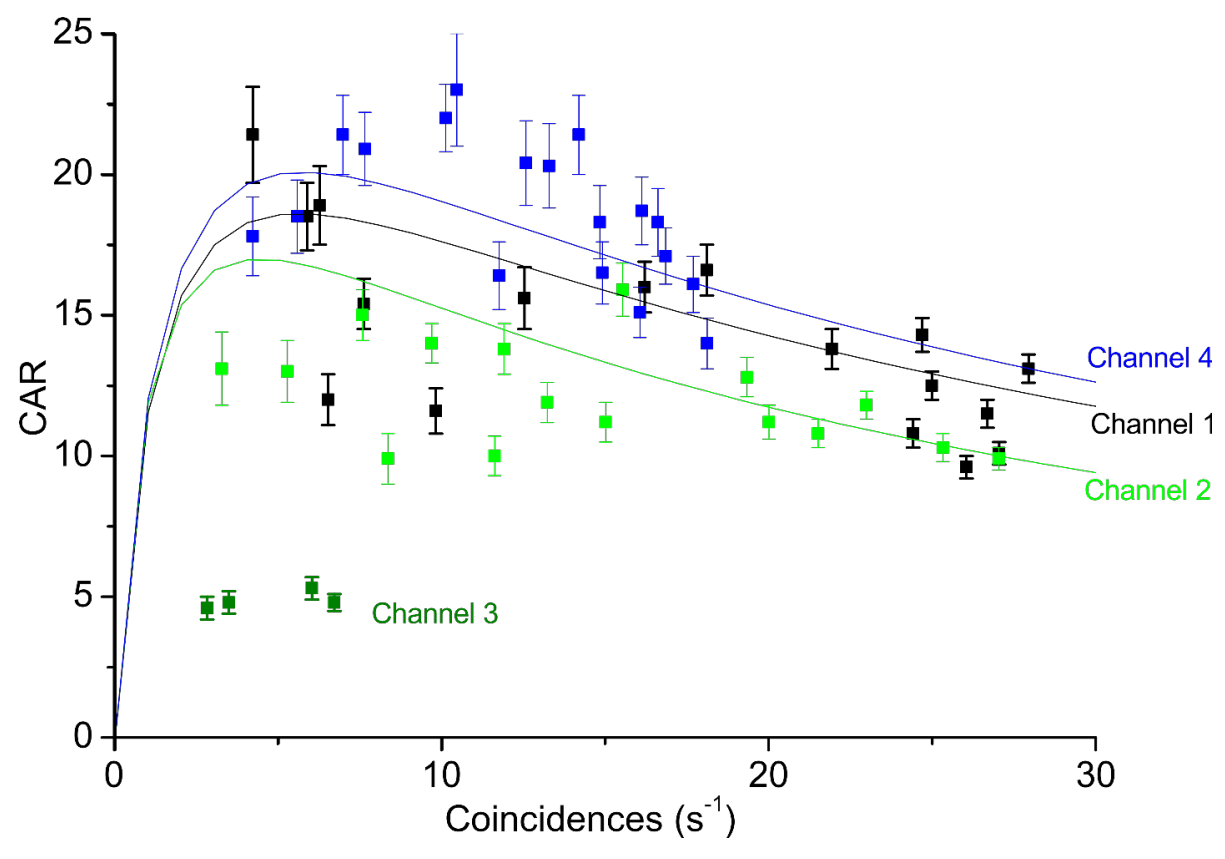

Figure 7.7: The registered $C A R$ as a function of the coincidence rate for individual PPLN waveguide. The solid lines represents the estimated theoretical $C A R$ using the data presented in table 7.1. 


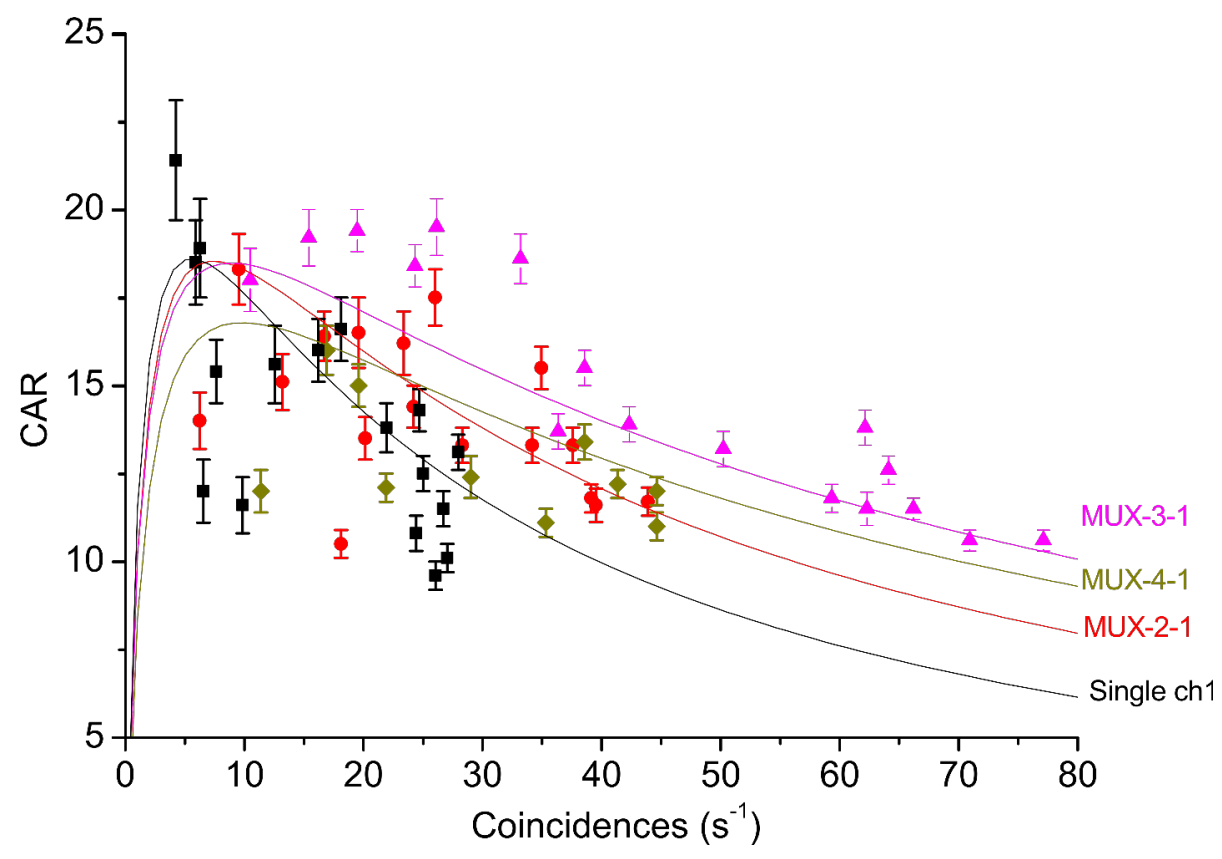

Figure 7.8: The registered $C A R$ as a function of the coincidence rate for multiplex PPLN configurations. The solid lines represents the estimated theoretical $C A R$ using the data presented in table 7.1.

Table 7.1: For each channel associated with different PPLN waveguides, the calculated photon pair production rate, the maximum measured $C A R$, losses for signal and idler arms $\left(\eta_{s, i}\right)$, heralding detector efficiency $\left(\eta_{\text {det }}\right)$ and the corresponding dark count rates for the heralding detector $\left(d_{\text {det }}\right)$ are shown below.

\begin{tabular}{lcccc}
\hline Single channel & 1 & 2 & 3 & 4 \\
\hline$\mu\left(\times 10^{-3}\right)$ & 12.8 & 23.1 & 1.9 & 10.8 \\
Max. $C A R$ & 21 & 15 & 7 & 25 \\
\hline$\eta_{s}(\mathrm{~dB}$ incl detector $)$ & 33 & 33.5 & 32 & 33.1 \\
$\eta_{i}(\mathrm{~dB}$ incl detector $)$ & 19 & 21 & 26 & 20.5 \\
$\eta_{\text {det }}(\%)$ & 17.5 & 17.5 & 7.5 & 12.5 \\
$d_{\text {det }}(\mathrm{KHz})$ & 1.8 & 1.5 & 2 & 1 \\
\hline
\end{tabular}




\subsection{Conclusion and perspectives of the source}

We have successfully interfaced waveguides on a single PPLN chip with FLDW chips to produce a spatially multiplexed heralded single photon source. With proper fiber components, namely polarization controllers and optical variable delay line, the indistinguishability of the heralded photons could be easily engineered thus making them suitable for various quantum information protocols. Moreover, the produced heralded photons are tunable across the communication band of $1520-1580 \mathrm{~nm}$. Active spatial multiplexing is demonstrated with ease thanks to electronically controlled, fast optical switches. Compared to recent integrated spatial multiplexing demonstration [Collins et al. 2013], our heralded photon rates are an order of magnitude higher and we almost double the rate of another previous demonstration reported in [Xiong et al. 2013]. Again, we stress that this particular hybrid integration proves the scability our technique.

One of the element that bring big differences in the measured $C A R$ is the loss. In present condition, the breakdown of the losses for different components are as follows: PPLN $\rightarrow$ FLDW coupling $(3 \mathrm{~dB})$, FLDW WDMs $(3 \mathrm{~dB})$, combined circulators and FBGs ( $3 \mathrm{~dB})$, pump suppression filter $(1 \mathrm{~dB})$, variable optical delays $(1.5 \mathrm{~dB})$, in-line polarization controllers $(1 \mathrm{~dB})$ and bandpass filters $(3.5 \mathrm{~dB})$. By replacing some of the components with the better ones will improve the overall performance of our source. It is also important to have an array of heralding detectors exhibiting the same characteristics in term of the dark counts to avoid the use lower efficiency mode. Moreover, the replacement of the PPLN sample with a new one exhibiting better performances would further enhance the results. Future pigtailing of the $1 \rightarrow 4$ waveguide splitter can involve index matching adhesives which is less prone to the temperature fluctuations thus provides better stability in term of the coupling. Furthermore, the loss contributed by the filtering of signal and idler photons can be improved by choosing filters with appropriate acceptance bandwidth. Ideally, the bandwidth of the $1548 \mathrm{~nm}$ filter should be equal or greater than the sum of the pump and the $1312 \mathrm{~nm}$ filter bandwidth (see Chapter 8). The main goal of any single photon source is to produce indistinguishable photons needed by most quantum information experiment. In order to validate this, we plan to perform a Hong-Ou-Mandel experiment. This is a crucial step in proving spatial multiplexing as an valuable strategy in building a high-performance 
single photon source. It would be possible to perform a Homg-Ou-Mandel experiment [Hong et al. 1987] so as to verify the indistinguishability in term of the produced single photons. 



\section{Ultra-fast heralded single photon}

\section{source based on telecom technologies}

\section{Contents}

8.1 Inspirations from the telecom industry . . . . . . . . 155

8.2 Ultra-fast generation of heralded single photons: the experiment 157

8.2.1 Efficient sequential non-linear, frequency conversion stages . . . . . . 158

8.2.2 Deterministic photon pair separation . . . . . . . . . . . . . . . . 162

8.2.3 Detection of heralding photons in $\mathrm{MHz}$ regime $\ldots \ldots \ldots$. . . . 163

8.2.4 Associated losses due to spectral filtering of photons . . . . . . . . . 164

8.3 Characterizing the quality of single photon sources via HBT $\ldots 166$

8.4 Conclusion and perspectives $\ldots \ldots \ldots \ldots \ldots \ldots$

Some results presented in this chapter were published in L. A. Ngah et al., Ultra-fast heralded single photon source based on telecom technology, Laser \& Photonics Reviews, Volume 9, Issue 2, pages L1-L5, March 2015.

\subsection{Inspirations from the telecom industry}

In this few decades, the advances in telecom industry are overwhelming and the industry is still moving forward with significant pace. Through this considerable technological growth, people are benefiting, for instance, from the incredibly high-rate and long distance connectivity via worldwide networks of low-loss optical fibers. Cutting-edge technologies such as ultra-fast telecom lasers with up to tens of Gigabit/s repetition rate or dense wavelength division multiplexing (DWDM) technology play vital roles towards current achievements 
and are believed to continue to be the backbone for future telecom networks. These high repetition rate lasers are responsible to drastically speed up the connectivity. In parallel, with DWDM technology, "fiber exhaust" issue can be rectified, thus more and more data can be transmitted on a single telecom fiber. We exploit, in a particular way, the combined advantages offered by the telecom technology and those of non-linear optics devices such as periodically poled lithium niobate waveguides (PPLN/W) presented in previous chapters, and we demonstrate an ultra-fast heralded single photon source (UFHSPS).

Most of the demonstrated heralded single photon sources (HSPS) at telecom wavelength in pulsed regime rely on visible pump fields originated from solid-state lasers which are later responsible for the birth of photon pairs by spontaneous parametric down conversion (SPDC) process in non-linear crystals. As a matter of fact, SPDC is a probabilistic process, as has been discussed in Chapter 6 with the statistic of the generated photon pairs follows either Poisson or thermal distribution. Accordingly, the mean number of photon pairs produced per pump pulse $\bar{n}$ needs to be maintained well below 0.1 in order to guarantee negligible multi-photon contributions [de Riedmatten et al. 2004b, Jin et al. 2014]. Furthermore, solid-state lasers are limited in term of the operational repetition rate up to only a few hundreds of MHz. This, combined with low $\bar{n}$ requirement, has, a matter of fact, considerably limited the speed of previous demonstrated HSPSs. Due to the factors mentioned above, researchers tend to focus most of their efforts towards:

- Increasing the SPDC efficiency by engineering ultra-bright sources [Mosley et al. 2008a, Krapick et al. 2013];

- Optimizing photon pair collection, separation, and propagation downstream the SPDC stage [Pomarico et al. 2012, Da Cunha Pereira et al. 2013, Ramelow et al. 2013, Guerreiro et al. 2013].

As has been discussed in Chapter 6, speeding up the available rate of heralded photons can be achieved by spatial multiplexing of the pump beam over many simultaneously SPDC stages [Migdall et al. 2002, Meany et al. 2012, Collins et al. 2013, Xiong et al. 2013]. As an alternative, the key feature in our UFHSPS is the use of a state-of-the-art, ultra-fast telecom laser operating at $10 \mathrm{GHz}$ repetition rate, to passively time multiplex the pump beam of a single SPDC process. Let us consider the following general formula concerning the detection 
rate of generated single photons in pulsed regime:

$$
R=f \cdot \bar{n} \cdot \mu \cdot \eta_{\text {det }} .
$$

Here, $f$ denotes the laser repetition rate, $\bar{n}$ depicts the mean number of emitted single photons, $\mu$ represents the overall single photon transmission, and $\eta_{\text {det }}$ is the quantum detection efficiency of the employed single photon detector. As shown by Eq.(8.1), the detection rate $R$ can be considerably increased proportionally to the repetition rate of the pump laser. In particular, high repetition rate lasers allow generating photon pairs at high rate while maintaining $\bar{n}$ as low as possible, as required for negligible multi-photon generation events [Zhang et al. 2007, Broome et al. 2011, Morris et al. 2014].

So far, we have explained the notion of passive temporal multiplexing in pulsed regime. In Sec.8.2, we will describe the development of our ultra-fast heralded single photon source based on the latter.

\subsection{Ultra-fast generation of heralded single photons: the ex- periment}

The overall experimental setup of the UFHSPS is depicted in Fig.8.1. In the following subsections, all the necessary development stages of our source will be thoroughly described. This includes:

- Efficient non-linear frequency conversion stages towards generating photon pairs at telecom wavelength around $1540 \mathrm{~nm}$. Additionally, the normalized brightness $B$ and the SPDC efficiency $\eta_{S P D C}$ for our photon pair generator are characterized.

- Low-loss, deterministic photon pair separation with the help of two conventional telecom optical filters.

- The use of fast superconducting single photon detector (SSPD) in order to detect the heralding photons in $\mathrm{MHz}$ regime.

- Associated losses due to spectral filtering of the generated signal and idler photons. 
Later in Sec.8.3, a Hanbury Brown and Twiss (HBT) experimental setup towards characterizing the performance of our UFHSPS in term of the second-order correlation function of $g^{(2)}(0)$ is outlined.

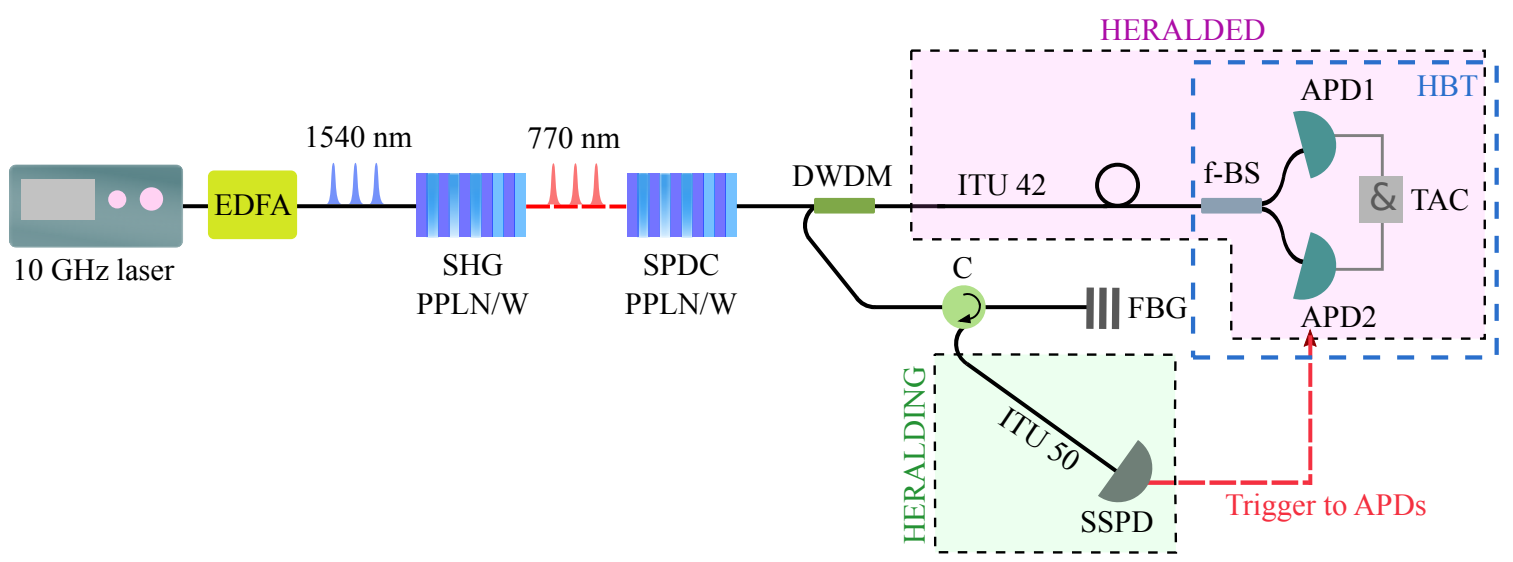

Figure 8.1: Schematic setup of the ultra-fast heralded single photon source. EDFA (erbium doped fiber amplifier), PPLN/W (periodically poled lithium niobate waveguide), SHG (second harmonic generation), SPDC (spontaneous parametric down conversion), DWDM (dense wavelength division multiplexer), C (optical circulator), FGB (fiber Bragg gratings filter), BS (fiber beamsplitter), SSPD (superconducting single photon detector), APD (avalanche photodiode). (b): The emission spectrum of the SPDC centered around $1540.56 \mathrm{~nm}$. By using a combination of FBG and DWDM, signal (ITU 50) and idler (ITU 43) photons are deterministically separated fom the pair.

\subsubsection{Efficient sequential non-linear, frequency conversion stages}

In our setup, we employ a $10 \mathrm{GHz}$ repetition rate telecom laser (Pritel UOC). This laser delivers 2 ps light pulses at $1540.56 \mathrm{~nm}$, with an emission spectrum bandwith of $1.5 \mathrm{~nm}$ (see Fig.8.2). We note that due to SPDC phase matching condition for generating both signal and idler photons in telecom C-band of wavelengths, a second harmonic generation (SHG) stage is indispensable to convert the pump laser light from $1540 \mathrm{~nm}$ to $770 \mathrm{~nm}$.

The employed experimental setup in order to observe, both SHG and SPDC emission spectra, is illustrated in Fig.8.3. $1540.56 \mathrm{~nm}$ light form $10 \mathrm{GHz}$ Pritel laser is first amplified by an erbium doped fiber amplifier (EDFA). Towards achieving frequency doubling, the amplified light is then passed through a polarization controller (PC) before being injected into a $1 \mathrm{~cm}$ long, type-0 PPLN/W. Using the PC, pump light is vertically polarized, so as to fulfill the QPM. With the help of a flipping mirror, the frequency doubled light is then 


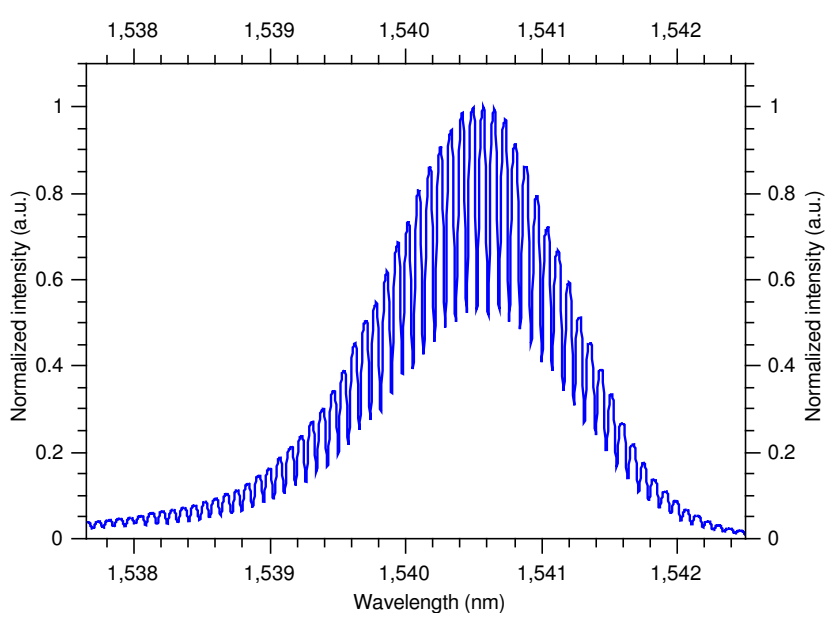

Figure 8.2: Emission spectrum of Pritel UOC when operating at $10 \mathrm{GHz}$ repetition rate. For this laser, the full width at half maximum (FWHM) is measured to be about $1.1 \mathrm{~nm}$ and the spectrum is centered around $1540.56 \mathrm{~nm}$.

directed towards an optical spectrum analyzer (Anritsu MS9710B) for spectrum measurement. The observed SHG spectrum shown in Fig.8.4 (a) is centered around the expected value of $770.2, \mathrm{~nm}$. Its FWHM is measured to be $0.4 \mathrm{~nm}$, as expected for Fourier transform limited pulses of 2 ps duration. Such a phase matching condition is achieved through the use of a waveguide having a width of $6 \mu \mathrm{m}$ and a poling period of $15.7 \mu \mathrm{m}$. Furthermore, the fine tuning of the intended phase matching condition is achieved by stabilizing the temperature of the SHG PPLN/W around $112^{\circ} \mathrm{C}$. The PPLN/W is fabricated using our expertise in Nice, exploiting the use of the so-called soft proton exchange technique [Tanzilli et al. 2002]. Additionally, for a maximum $\sim 1 \mathrm{~W}$ of infrared pump power, the intensity of the frequency doubled light is measured to be $98 \mathrm{~mW}$ which corresponds to a conversion efficiency of around 9.8\%/W.

Subsequent to the SHG stage, another home-made PPLN/W is installed. This $2 \mathrm{~cm}$ long, type-0 PPLN/W is injected with the frequency doubled light at $770.28 \mathrm{~nm}$. SPDC, which is the inverse process of SHG, generates, inside the PPLN/W, photon pairs at telecom C-band of wavelengths. For the SPDC spectrum measurement, down-converted photons are collected with a standard single mode fiber butt-coupled to the output facet of the corresponding waveguide. The collected photons are then sent to a tunable filter (Yenista XTM-50). The transmission bandwidth of the filter is set to $\sim 1 \mathrm{~nm}$, thus providing sufficient 


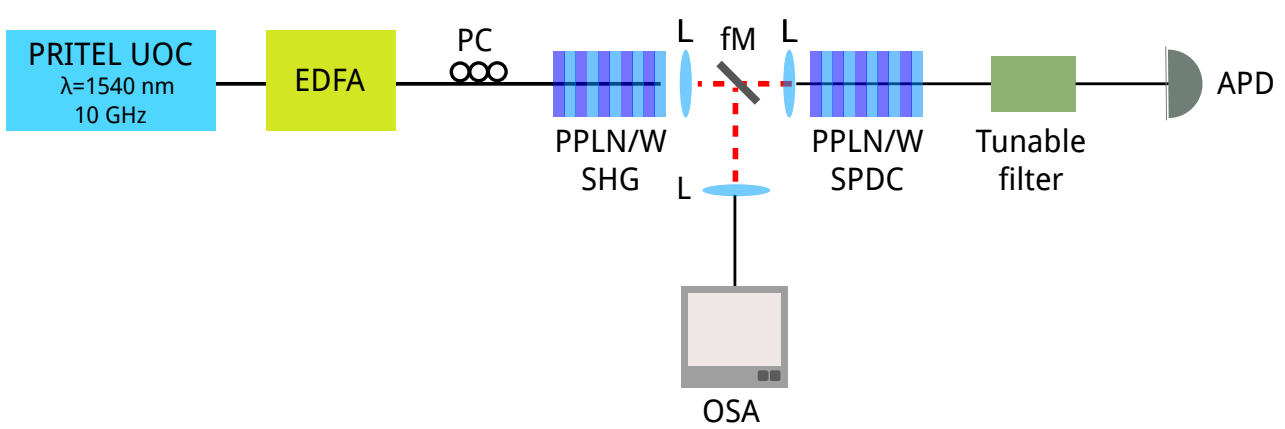

Figure 8.3: Experimental setup for both SHG and SPDC characterizations. $1540.56 \mathrm{~nm}$ light form $10 \mathrm{GHz}$ Pritel laser is first amplified by an erbium doped fiber amplifier (EDFA). The amplified pump light is then passed through a polarization controller (PC) before being injected into a type-0 SHG PPLN/W. A flipping mirror (fM) is used to route the frequency doubled light towards an optical spectrum analyzer (OSA). With the OSA, SHG emission spectrum is registered. For SPDC characterization, the frequency doubled light is directly injected directly into another home-made type- 0 PPLN/W. The generated photon pairs via SPDC in this particular PPLN/W are collected by a single mode fiber and sent towards a tunable filter, followed by an avalanche photo-diode (APD, free-running IDQ 220). The photons are then counted as a function of the wavelength given by the tunable filter, thus revealing the SPDC emission spectrum.

resolution and adequate number of photons for fast SPDC spectrum acquisition. Photons transmitted by the filter are then detected by a free-running APD (IDQ 220). Here, the photons are counted as a function of the wavelength given by the tunable filter. The phase matching condition for the SPDC is obtained through the use of a PPLN/W featuring a width of $5 \mu \mathrm{m}$ and a poling period of $16.3 \mu \mathrm{m}$. The PPLN/W is also temperature stabilized at $96.42^{\circ} \mathrm{C}$. As can be seen in Fig.8.4 (b), a broad SPDC emission bandwidth of about $80 \mathrm{~nm}$ with the degeneracy of $1540.56 \mathrm{~nm}$ is observed. In this context, the term degeneracy refers to the condition of both idler and signal photons emitted at the same frequency.

Interestingly, by extending the experimental setup shown in Fig.8.3 to that of Fig.8.5, we can easily estimate the brightness $B$ as well as the SPDC $\eta_{S P D C}$ efficiency of our photon pair generator. We note that the measurement procedure is very similar as the one described in Chapter 4 of this manuscript. In this case, the generated photon pairs are sent to the same Yenista filter. Only this time, the central wavelength of the filter is fixed at $1540.56 \mathrm{~nm}$ corresponding to the degenerate wavelength of the produced photon pairs. The transmission bandwidth of such filter is maintained at $1 \mathrm{~nm}$ large. After the filtering stage, a fiber beam splitter (f-BS) is employed to probabilistically separate the paired photons whose outputs 
(a)

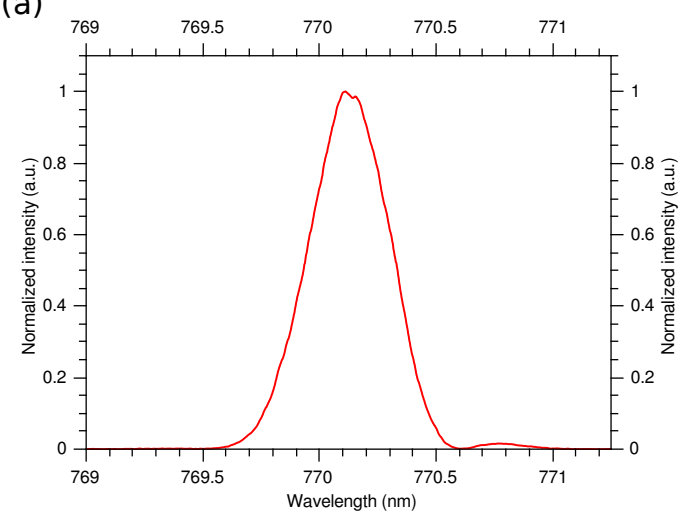

(b)

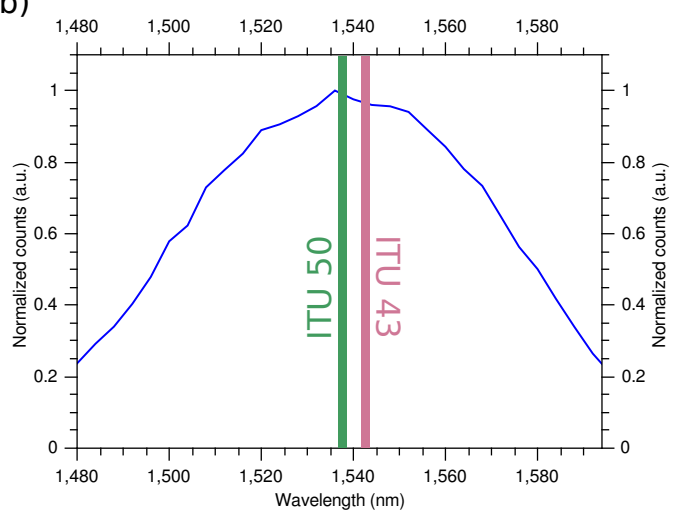

Figure 8.4: (a): SHG spectrum centered around $770.28 \mathrm{~nm}$ with an FWHM of $0.4 \mathrm{~nm}$. (b): Emission spectrum of SPDC exhibiting a large spectral bandwidth of about $80 \mathrm{~nm}$. The spectrum is centered around $1540.56 \mathrm{~nm}$.

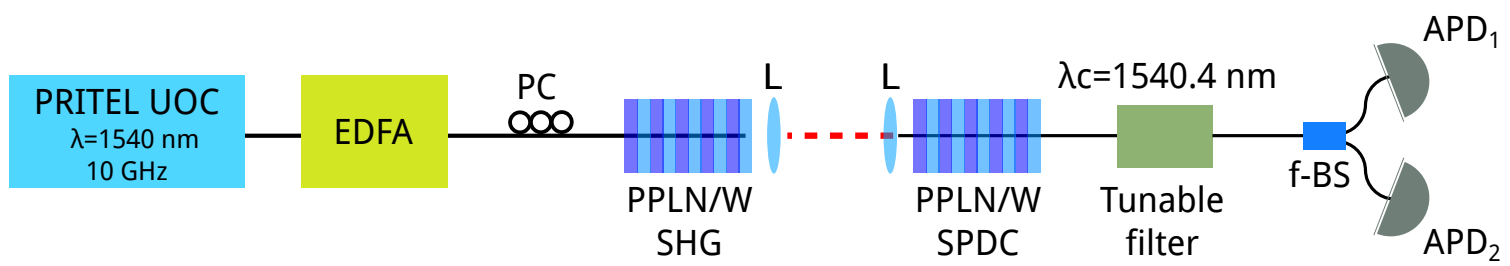

Figure 8.5: Experimental setup used for characterizing the normalized brightness $B$ and the SPDC efficiency $\eta_{S P D C}$ of the type-0 photon pair generator. After the SPDC stage, the generated photon pairs are subjected, again, to the Yenista filter whose central wavelength is fixed at $1540.56 \mathrm{~nm}$. After the filtering stage, a fiber beam splitter (f-BS) is employed to probabilistically separate the paired photons. Two avalanche photo-diodes (APD) are employed to detect the separated paired photons.

are connected to two APDs (free-running IDQ 220 featuring 20\% of quantum detection efficiency and $\sim 10^{-6} / n s$ dark count probability). Accordingly, the recorded singles $S_{1}$ and $S_{2}$ on detectors $A P D_{1}$ and $A P D_{2}$, respectively, read

$$
\begin{aligned}
S_{1} & =\frac{1}{2} \mu_{1} \eta_{1} N_{\text {pair }}, \\
S_{1} & =\frac{1}{2} \mu_{1} \eta_{1} N_{\text {pair }} .
\end{aligned}
$$

Here, $\mu_{1}$ and $\mu_{2}$ represent the overall transmission from the SPDC PPLN/W to $A P D_{1}$ and $A P D_{2}$, whose the quantum detection efficiency is denoted by $\eta_{1}$ and $\eta_{2}$, respectively.While $N_{\text {pair }}$ represents the amount of photon pairs being generated per second, and the coincidence 
rate $C$ between the two detectors is given by

$$
C=\frac{1}{2} \mu_{1} \eta_{1} \mu_{2} \eta_{2} N_{\text {pair }}
$$

Eventually, we obtain

$$
N_{\text {pair }}=\frac{2 S_{1} S_{2}}{C}
$$

In our case, the recorded singles and coincidence rate prompt an $N_{\text {pairs }}$ of $1.53 .10^{6} \mathrm{~s}^{-1}$. Thus, this leads to a brightness of

$$
B=\frac{2.5 \cdot 10^{5}}{\mathrm{~mW} . \mathrm{s.GHz}}
$$

and to an SPDC efficiency of

$$
\eta_{S P D C}=6.4 \cdot 10^{-7} \frac{\text { pairs }}{\text { pump photon }}
$$

(see Sec.4.2.6).

\subsubsection{Deterministic photon pair separation}

Photon pairs at telecom wavelength are available after the SPDC stage. As per heralding scheme requirement, the idler and signal photons must be separated from the pair. To do so, we take advantage of a combination of standard $200 \mathrm{GHz}$ dense wavelength division multiplexing (DWDM) and fiber Bragg grating (FBG) telecom filters to perform the so-called low-loss deterministic photon pair separation. Both filters exhibit flat-top type acceptance and the responses in spectrum is shown in Fig.8.6. This filter combination allows picking up, within the SPDC spectrum, signal and idler photons slightly away from perfect degeneracy and thus deterministically separable. By referring to the full experimental setup shown in Fig.8.1 (a), photon pairs generated by the SPDC stage are first directed towards a DWDM filter where the heralding idler photons are selected at $1543.73 \mathrm{~nm}$ (ITU 42) within a bandwidth of $1.2 \mathrm{~nm}$. The selected idler photons are then transmitted towards a fiber Hanbury Brown and Twiss (HBT) type setup [Hanbury Brown \& Twiss 1956]. Meanwhile, being reflected by the DWDM filter, the heralded signal photons counterparts at $1537.4 \mathrm{~nm}$ 
(ITU 50) are eventually picked by the FBG filter with an acceptance bandwidth of $250 \mathrm{pm}$. The mapping of the mentioned ITU channels 42 and 50 on the SPDC emission spectrum is illustrated in Fig.8.4 (b). Concerning the photon pairs generated by our SPDC stage, the used of optical filters is not just limited to gain certain coherence time for purity required by various quantum protocols, but also to prevent the saturation of the employed single photon detectors due to ultra-broad SPDC emission in the frequency domain.
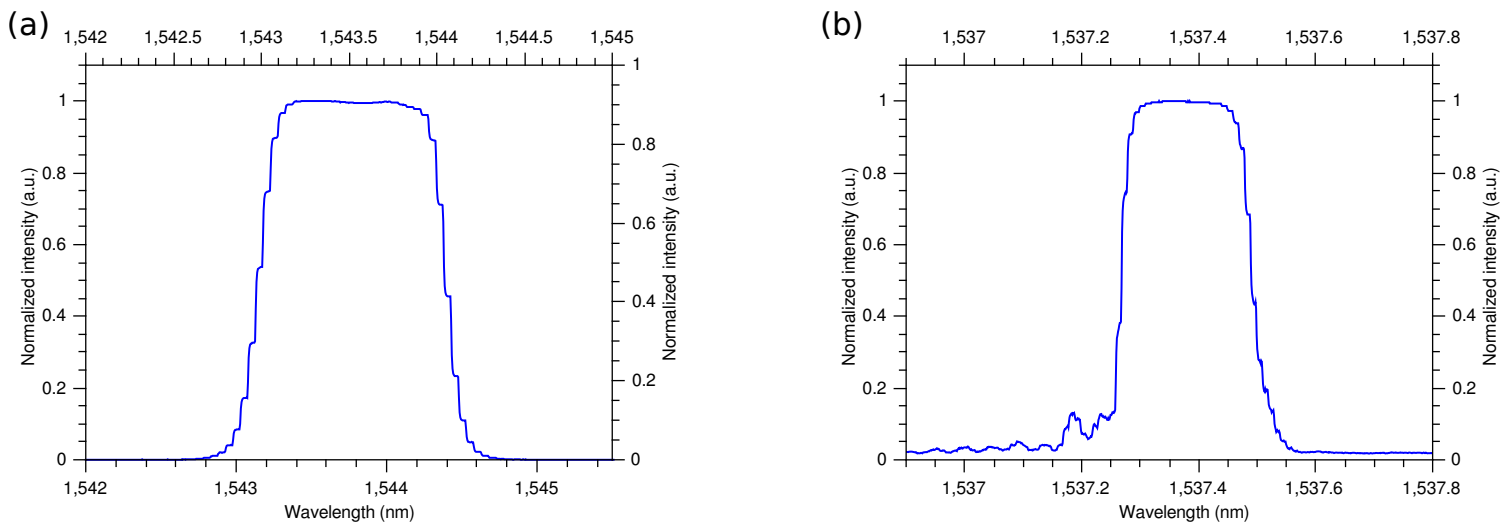

Figure 8.6: Typical response in spectrum of (a): DWDM ITU 42 filter and (b): FBG ITU 50 filter. The measured FWHM for both filters are $1.2 \mathrm{~nm}$ and $250 \mathrm{pm}$, respectively. The spectrum of the DWDM filter is centered around $1543.73 \mathrm{~nm}$ while the FBG one is centered at $1537.4 \mathrm{~nm}$.

\subsubsection{Detection of heralding photons in $\mathrm{MHz}$ regime}

Signal photons which are earlier picked by the FBG filter (ITU 50) are directed towards the so-called heralding photon path and they are eventually detected by a superconducting single photon detector (SSPD, SCONTEL LTD 24/30-008).

Compared to other commercially available, off-the-shelves single photon detectors at telecom wavelength, e.g. InGaAs APDs, the use of SSPD is critical to our experiment due to the following reasons:

- The SSPD exhibit a dead time $<2$ ns, i.e. extremely short recovery time and it provides afterpulsing-free environment. Just to give an example, typical InGaAs APDs at telecom wavelength have a dead time around a few of $\mu$ s and due to afterpulsing, the detection rate is limited, typically, around $100 \mathrm{kHz}$. 
- Low dark counts of $<100 \mathrm{~Hz}$ allow the use of the SSPD in gated-free, i.e. free-running mode which is crucial as there are no commercial InGaAs APDs that can be gated in the $\mathrm{GHz}$ regime.

- SSPD is well known to have the best timing jitter among in-house and commercially available single photon detectors at telecom wavelength. In the gigahertz regime, inadequate timing jitter can give rise to intersymbol interference phenomena [Gordon et al. 2005, Takesue et al. 2007a, Clarke et al. 2011] which leads to erroneous counts due to detection error is bigger then the pulse separation.

Our SSPD exhibits a quantum detection efficiency $\eta_{S S P D}$ of 0.17 and a measured timing jitter $\lesssim 57$ ps. Collectively, thanks to extremely short dead time and short timing jitter, this type of detector is capable of detecting single photons up to $10 \mathrm{MHz}$ counting rates. A brief information on the working principle of an SSPD is documented in annex 1.

The first figure-of-merit associated with our UFHSPS is the detection rate of the SSPD, i.e. the heralding rate $R_{H}$. Besides $\eta_{S S P D}$, in the limit of negligible dark counts, $R_{H}$ also depends on the laser repetition rate $f$ and on the photon pair generation probability, thus leading to

$$
R_{H}=f \cdot \bar{n} \cdot \gamma \cdot T_{H} \cdot \eta_{S S P D}
$$

Here, $\bar{n}$ represents the mean number of emitted heralding signal photons per pump pulse bounded by the applied filtering spectral bandwidth. Furthermore, $\gamma$ denotes the coupling efficiency between the SPDC waveguide and the collection fiber at its output, and $T_{H}$ stands for the transmission from the collection fiber to the SSPD.

\subsubsection{Associated losses due to spectral filtering of photons}

After the output of the collection fiber, our measured propagation losses are $2.5 \mathrm{~dB}$ on the signal path and $1.9 \mathrm{~dB}$ on the idler one. These values include the non-unitary transmission of employed optical filters and other telecom components. Beside these contributions and depending on the spectral filtering conditions, extra losses can be introduced by what we call the "filtering factor".

As has been already discussed, during the creation of photon pair by SPDC process, a 
pump photon is annihilated in order to give birth to a photon pair comprising a signal and an idler photon whose combined energy is equal to the energy of the original pump photon. The conservation of energy can be written as

$$
\omega_{p}=\omega_{s}+\omega_{i}
$$

where $\omega$ denotes the photon angular frequency.

As the pump has a certain spectral width, this leads to spectral width of signal photons of

$$
\Delta \omega_{s}=\Delta \omega_{i}+\Delta \omega_{p}
$$

In our case, the detection of a signal photon is used to herald the presence of an idler photon. For practical applications, heralded idler photons need to be further spectrally filtered. We indicate as $\Delta \omega_{f_{i}} \leq \Delta \omega_{i}$ the filter bandwidth acting upon the heralded photons. As a matter of fact, any signal photons phase-matched with the idler ones within $\omega_{i} \pm \Delta \omega_{f_{i}}$ must be considered. This imposes the filtering of signal photons within the bandwidth of $\Delta \omega_{s}$ given by

$$
\Delta \omega_{s}=\Delta \omega_{f_{i}}+\Delta \omega_{p}
$$

By taking the above into consideration, a filtering factor $\mathcal{F}$ is generally introduced:

$$
\mathcal{F}=\frac{\Delta \omega_{f_{i}}}{\Delta \omega_{f_{s}}+\Delta \omega_{p}} .
$$

Here, $\mathcal{F}<1$ simply implies extra losses to the experimental setup due to imperfect choice of $\Delta \omega_{f_{s}}$. For our experiment, the term $\Delta \omega_{p}$ can be read directly from Fig.8.4 (a). In the same manner, $\Delta \omega_{f_{s}}$ and $\Delta \omega_{f_{i}}$ can be obtained from Fig.8.6 (a) and (b) respectively. In our case, $\Delta \omega_{p}, \Delta \omega_{f_{s}}$ and $\Delta \omega_{f_{i}}$ are calculated to be $202 \mathrm{GHz}, 32 \mathrm{GHz}$ and $151 \mathrm{GHz}$, respectively. By plugging in all the calculated values into Eq.(8.12), we obtain a filtering factor $\mathcal{F}>1$. This confirms that no extra losses are acquired from the filtering on both signal and idler photons. 


\subsection{Characterizing the quality of single photon sources via HBT}

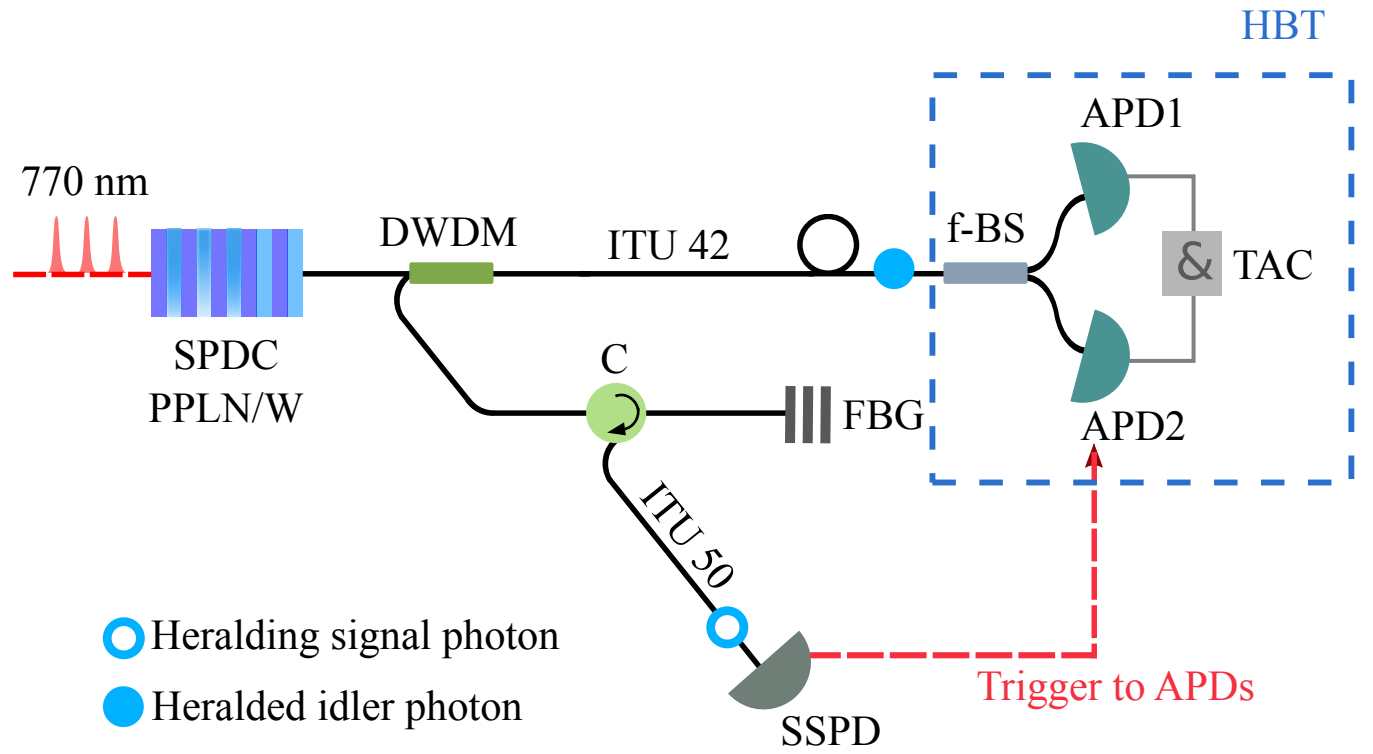

Figure 8.7: The fiber Hanbury Brown and Twiss (HBT) setup comprising a fiber beam splitter and two avalanche photo-diodes (APD) is outlined.

Heralded idler photons are directed towards the fiber Hanbury Brown and Twiss (HBT) setup comprising a fiber beam splitter and two detectors. Here, heralded photons are characterized in terms of $P_{1}$ and $g^{(2)}(0)$ (see Fig.8.7). $P_{1}$ represents the probability of observing one heralded photon per heralding event while the second-order correlation function $g^{(2)}(0)$ is responsible to quantify the singleness of the heralded photons. As has been discussed in Sec.8.2.4, the heralded photon bandwidth has been chosen so as to adequately collect the idler photons who are energy-matched with the heralding ones given the spectral width of the SPCD pump. At both outputs of the HBT, two detectors, $A P D_{1}$ (IDQ 210) and $A P D_{2}$ (IDQ 201) are employed. Both detectors exhibit each a detection efficiency $\eta_{1,2}$ of $25 \%$, a timing jitter of $\sim 120 \mathrm{ps}$, and a dark count probability of $10^{-6} / \mathrm{ns}$.

$\mathrm{APD}_{1}$ is externally triggered by the detection signal from the $\mathrm{SSPD}$, while $\mathrm{APD}_{2}$ is triggered by $S_{1}$, the detection signal of $\mathrm{APD}_{1}$. Accordingly, the detection rate of $\mathrm{APD}_{2} S_{2}$ gives the coincidence signal between the two APDs. In order to reduce the contribution of accidental detection of signal and idler photons generated by different pump pulses, at 
the output of each APD, we use a time-to-amplitude converter (TAC, Ortec 567) provided with single channel analyzer (SCA) option so as to post-select detection events within a narrow time window after the trigger signal. Ideally, optimal filtering is achieved by an SCA detection window shorter than the delay between subsequent pump pulses, i.e. 100 ps. Experimentally, however, the accuracy on the detection time is essentially down-limited by the convolution of the detectors' timing jitters of $\sqrt{120^{2} \mathrm{ps}+57^{2} \mathrm{ps}}=138 \mathrm{ps}$. Accordingly, in order to efficiently collect the coincidence events, we set for $\mathrm{APD}_{1}$ and $\mathrm{APD}_{2}, \mathrm{SCA}$ time windows of $\approx 300 \mathrm{ps}$ and $\approx 400 \mathrm{ps}$, respectively.

As detailed [Alibart et al. 2005], the values of $P_{1}, P_{2}$ and $g^{(2)}(0)$ can be expressed in terms of the conditional detection rates, $S_{1}$ and $S_{2}$, by taking into account the non-unitary detection efficiency of the APDs as well as their dark count rates. In the limit of negligible detector dark counts and of $S_{2} \ll S_{1}$ we obtain

$$
P_{1} \simeq \frac{2 S_{1}}{R_{H} \cdot \eta_{1}} \quad \text { and } \quad P_{2} \simeq \frac{2 S_{2}}{R_{H} \cdot \eta_{1} \cdot \eta_{2}}
$$

which then lead to the final approximate value of $g^{(2)}(0)$,

$$
g^{(2)}(0) \simeq \frac{R_{H} \cdot S_{2} \cdot \eta_{1}}{S_{1}^{2} \cdot \eta_{2}}
$$

where, in the actual data analysis, $R_{H}, S_{i}$ and $\eta_{i}(\mathrm{i}=1,2)$ are corrected by the effect of the APDs dead times (10 $\mu$ s for each APD) [Ramelow et al. 2013]. We stress that, compared to [Alibart et al. 2005] where the heralding detector is used to trigger both $\mathrm{APD}_{1}$ and $\mathrm{APD}_{2}$, in our configuration, $A P D_{2}$ is triggered by the detection of $A P D_{1}$. In this case, Eq.(8.13) (8.14) take into account this modification. As the heralding efficiency, $P_{1}$, linearly depends on the transmission of the heralded photon path, $T$, and on the coupling efficiency, $\gamma, P_{1}$ can be equivalently expressed as $P_{1} \simeq \gamma \cdot T$. As shown in Fig.8.8 (a), in all of our tests, we measure $P_{1} \approx 0.42$, corresponding to an estimated $\gamma$ of 0.60 . Coupling efficiencies as high as 0.80 have been recently measured for SPDC in bulk crystals [Ramelow et al. 2013, Guerreiro et al. 2013], however our pertinent choice to use a non-linear interaction in optical waveguides presents the advantage of better SPDC efficiency together with a simplified and more stable setup. Besides, an improvement of our coupling efficiency could be obtained by 
AR coating the facets of our SPDC crystal [Krapick et al. 2013].

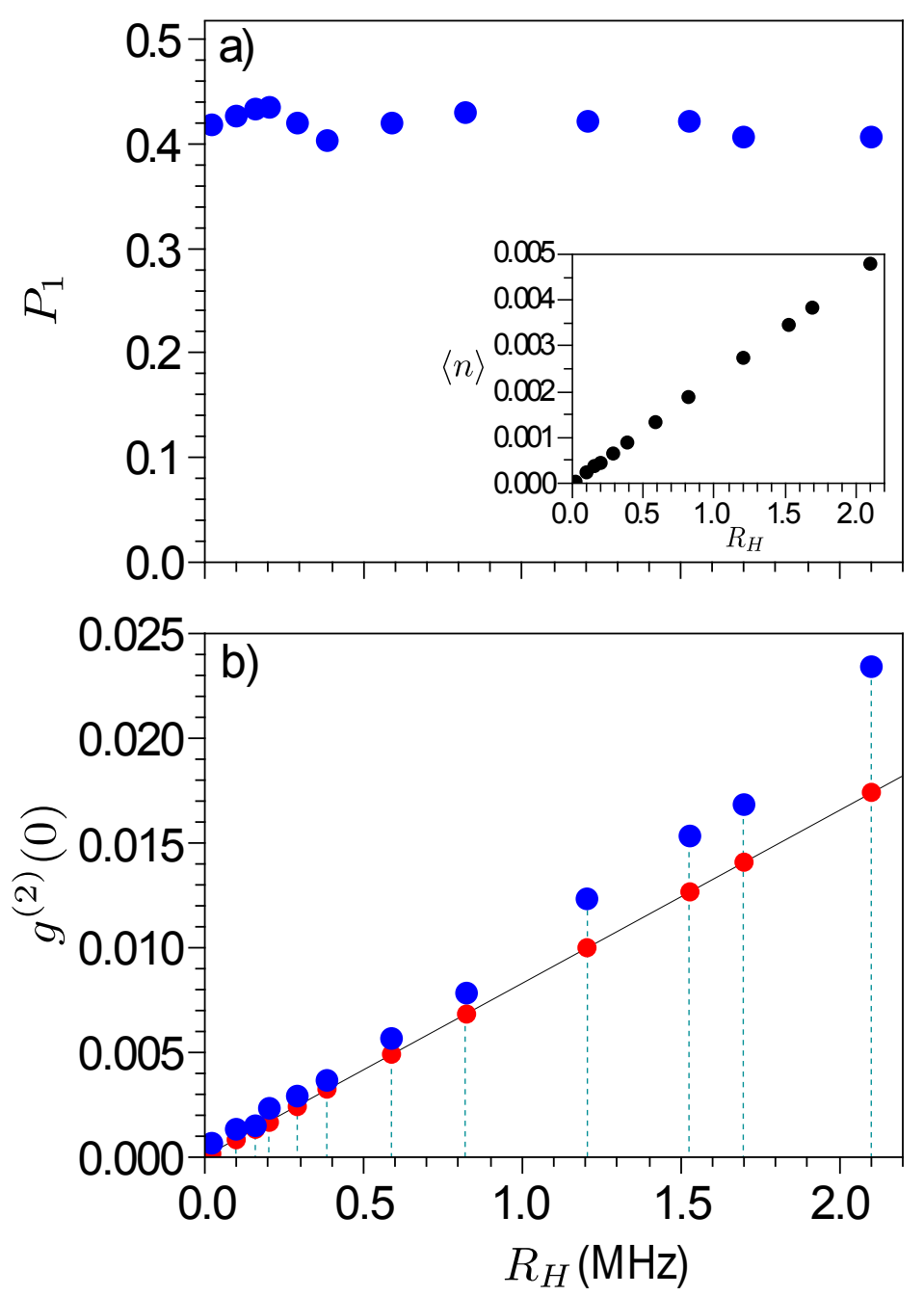

Figure 8.8: Experimental results for the heralding efficiency $P_{1}$ in (a) and for the heralded photon autocorrelation function $g^{(2)}(0)$ in (b), as functions of the rate of detected heralding photons, $R_{H}$. The inset of in (a) shows the mean number of produced pairs in the heralding photon bandwidth per pump pulse vs. $R_{H}$. In (b), the blue dots represent experimental data and the red dots depict the theoretically calculated $g^{(2)}(0)$ in the single mode theory (see [Sekatski et al. 2012], the line is a help to the eye).

In order to experimentally prove the ultra-fast operation of our source and the high quality of the produced single photons, we checked the evolution of our heralded states as a function of increasing values of the mean number of photon pairs generated. Variations of $\bar{n}$ can be simply obtained by increasing the power of the pump light at $770.28 \mathrm{~nm}$ at the input of the SPDC stage. The experimental values of $\bar{n}$ are then retrieved by inverting the 
Eq.(8.8) and dividing the corresponding $R_{H}$ by the laser repetition rate, and the overall collection, transmission and detection efficiency on the heralding photon path [Pomarico et al. 2012]. As already stressed, $\bar{n}$ calculated in this way coincides with the mean number of heralding photons generated per pump pulse.

$R_{H}$ is varied from a few tens of $\mathrm{kHz}$ up to $2.1 \mathrm{MHz}$, which corresponds to a maximum $\bar{n} \simeq 0.005$ (see inset of Fig.8.8 (a). Such values are well below the limit of 0.1 demanded for single photon states with negligible multi-photon contributions [de Riedmatten et al. 2004b]. This is confirmed by our experimental results for the autocorrelation function, $g^{(2)}(0)$. This quantity is ideally 0 for perfect single photon Fock states but increasing with the probability of unwanted multiple-photon events. Fig.8.8 (b) shows the measured $g^{(2)}(0)$ values (in blue) together with the theoretical prediction for a perfect single mode heralded photon (in red) [Sekatski et al. 2012]. Our highest experimental $g^{(2)}(0)$ is 0.023 , representing, to our knowledge, the best measured value for the autocorrelation function of heralded single photons announced at a rate in the $\mathrm{MHz}$ regime. The mismatch between the theory and the experiments observed for $R_{H} \geq 1 \mathrm{MHz}$ can be explained by non-negligible photonic noises due to unwanted time and spectral modes on the heralded photons. As discussed in [Mosley et al. 2008a, Mosley et al. 2008b], by choosing to collect all heralded photons, energy-matched with the heralding ones, we optimize the heralding efficiency $P_{1}$ at the price of a reduced heralded photon spectral purity (see also [Allevi et al. 2010]). The number of spurious spectral modes, $N_{f}$, is approximately given by the ratio between the heralded and heralding photon bandwidths, which is $\sim 8$ in our case. This value must be multiplied by the number, $N_{t}$, of parasite temporal modes which affect our $g^{(2)}(0)$ measurements and that can be estimated as the ratio between the detection window and the laser repetition rate, $\sim 4$ in our experiment. Accordingly, we find that in order to keep the mean number of heralded photon below 0.1, the mean number of heralding photon must be as low as $0.1 /\left(N_{f} \cdot N_{t}\right) \approx 0.003$, in agreement with a heralding rate of $\approx 1 \mathrm{MHz}$.

In Tab.A.1, we compare our results with those obtained in other recent implementations of HSPSs. As it can be seen, our results represent the best observed in terms of fast photon generation and low rate of multi-photon emissions. Besides, we recall that the experimental value of $R_{H}$ is proportional to the quantum efficiency of the heralding photon detector $\eta_{D}$. In our experimental setup, heralding photons are detected by an old-generation SSPD showing 
Table 8.1: Experimental results for different HSPSs realizations. For each group, only the values corresponding to the highest measured $R_{H}$ has been reported. ${ }^{a}$ theoretically calculated. ${ }^{b}$ estimated from reported data and $P_{1} .{ }^{c}$ expected values.

\begin{tabular}{lccccc}
\hline & $P_{1}$ & $\eta_{D}$ & $R_{H}$ & $\bar{n}$ & $g^{(2)}(0)$ \\
\hline Nice & 0.42 & 0.17 & $2.1 \mathrm{MHz}$ & 0.005 & 0.023 \\
Geneva [Pomarico et al. 2012]) & 0.45 & 0.50 & $4.4 \mathrm{MHz}$ & 0.1 & $0.18^{a}$ \\
Milan [Brida et al. 2012] & 0.13 & 0.40 & $\sim 10 \mathrm{kHz}$ & - & 0.0050 \\
Paderborn [Krapick et al. 2013] & 0.60 & 0.55 & $105 \mathrm{kHz}$ & - & 0.40 \\
Vienna [Ramelow et al. 2013] & 0.82 & 0.95 & $6 \mathrm{kHz}$ & - & - \\
Tokyo [Jin et al. 2014] & $<0.3$ & 0.70 & $\sim 150 \mathrm{kHz}$ & 0.00021 & - \\
Nice $^{c}$ & 0.5 & 0.90 & $15 \mathrm{MHz}^{b}$ & 0.005 & $\lesssim 0.020$ \\
\hline
\end{tabular}

a $\eta_{D} \simeq 0.17$. This value is well below the detection efficiency of Si-APDs used in many previous experiments [Brida et al. 2012, Pomarico et al. 2012, Krapick et al. 2013, Ramelow et al. 2013] which relied on non-degenerate emission of pairs of photon with heralding photons in the visible and NIR range of wavelengths. At the same time, we note that new generation SSPDs shows now quantum efficiency at telecom wavelength as high as $90 \%$ [Marsili et al. 2013, Yamashita et al. 2013]. Accordingly, by just replacing our SSPD with an improved one (and with no other fundamental change in the experimental setup), our observed $R_{H}$ values would be increased by a factor 5 without introducing any degradation to the value of $g^{(2)}(0)$. As already explained, a further improvement would be that of a better coupling efficiency at the SPDC output up to the optimal case $\sim 0.80$. For a comparison with results from other groups, the last line of Tab.8.1 reports expected results for our scheme, when using state-of-the-art SSPD detectors and exploiting optimized waveguideto-fiber coupling.

\subsection{Conclusion and perspectives}

In conclusion, we have employed advanced telecom technologies combined with nonlinear optics to realize an UFHSPS able to provide high-quality single photons at telecom wavelength by taking advantage of passive temporal multiplexing enabled by the use of state-of-the-art, ultra-fast telecom laser. In order to prove the high quality of our heralded single photons, we measured their characteristics, obtaining heralding rates up to $\mathrm{MHz}$ with $\bar{n}<0.1$ and second-order correlation function $g^{(2)}(0) \leq 0.023$. These results prove that our source is a 
valuable candidate for many quantum optics and quantum communication scheme where the requirement of fast data exchange and negligible-multiple photon emission are mandatory.

As has been discussed previously, noticeable improvement to the performance of our UFHSPS can be achieved by employing new generation SSPD featuring $90 \%$ quantum detection efficiency, and AR coating the facets of our SPDC crystal so as to optimize the waveguide-to-fiber coupling efficiency. 



\section{Part IV}

\section{Quantum networking based on entanglement swapping}





\section{All-optical synchronization for}

\section{quantum networking}

\section{Contents}

9.1 Theoretical framework $\ldots \ldots \ldots \ldots \ldots \ldots$

9.2 The quantum relay dilemma $\ldots \ldots \ldots \ldots \ldots \ldots$

9.3 Our approach: all-optical synchronization . . . . . . . . 181

9.3.1 Single, master optical clock operation . . . . . . . . . . . . . . 181

9.3.2 Hong-Ou-Mandel interference for validation . . . . . . . . . . . . . . 184

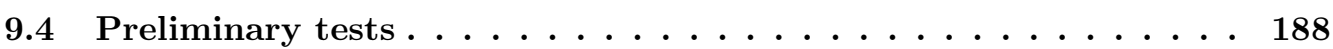

9.5 Towards the first prototype of HOM experiment $\ldots \ldots \ldots 191$

9.5.1 Setup for the two photon interference . . . . . . . . . . . . . 193

9.5.2 The path length matching procedure . . . . . . . . . . . . 195

9.5.3 Experimental results and discussions . . . . . . . . . . . . . . . . . 197

9.5.4 Conclusions and perspectives . . . . . . . . . . . . . . 199

As has been discussed before in Chapter 2, the achievable operational distance of quantum communication links is severely affected by losses in the transmission channels, as well as low detection efficiency and potentially high dark count rates of the employed single photon detectors. Aside from technological improvements, one can manipulate the theory of quantum physics for finding alternative solutions. This is exactly where This very fundamental, yet practical, and feasible approach relies on entanglement swapping [Zukowski et al. 1993, Gisin et al. 2002, Waks et al. 2002,Jennewein et al. 2002, Collins et al. 2005, Goebel et al. 2008, Ma et al. 2012b, Jin et al. 2015], i.e. teleportation of entanglement in a quantum 
relay configuration. Being an extension of the quantum teleportation protocol, entanglement swapping carries the same notion of benefiting the fact that one can cause two independent photons, i.e. sharing no common "quantum past" to become entangled.

The fully extensible architecture of a quantum relay (see Sec.2.1) is based on a simplistic approach of breaking a single, long quantum communication link onto several shorter sections. With each section is propagated by an entangled photon, the inter-quantumconnection between adjacent sections is established by performing the so-called Bell state measurement (BSM, also known as joint measurement) on the two inner photons coming from two distinct, remote entangled photon pair sources (see Fig.2.4). Consequently, these two photons are now projected onto an entangled state. Upon successful correlation detection, teleportation of entanglement to the remaining outer photons can be announced.

\subsection{Theoretical framework}

Considering the work of this thesis to demonstrate entanglement swapping between two remote communicating parties, say Alice and Bob, with time-bin entangled qubits, the following mathematical proofs throughout this section are tailored accordingly.

From now on,assume that an unbalanced Mach-Zehnder interferometer (see Fig.9.1), defined as the pump interferometer, splits incoming laser pulses, and subsequently generates, two new pulses with a fixed phase. This phase $\varphi$ is defined by the setting of this interferometer, which is the path length difference between the long and the short arms.

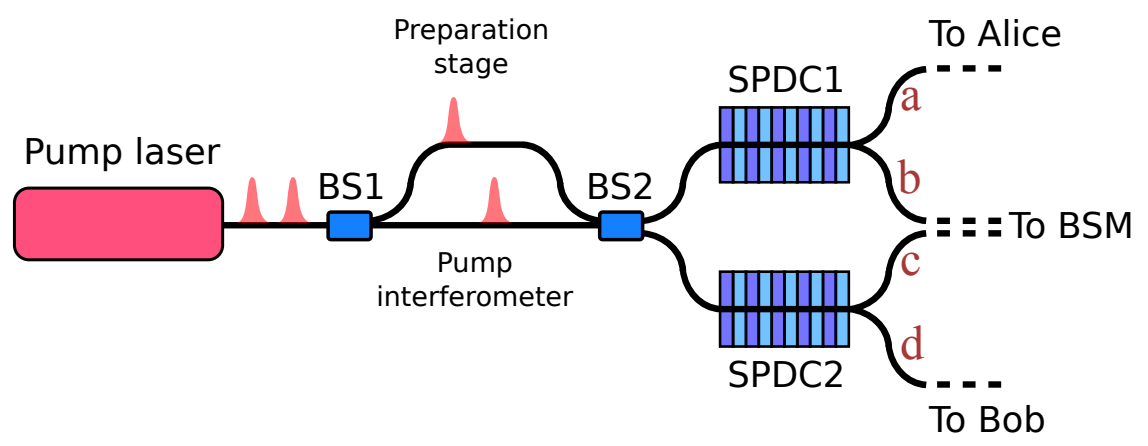

Figure 9.1: Simple configuration where a Mach-Zehnder interferometer is used to give back the coherence to the system due to the use of a pulsed laser. Photon pairs generated by both SPDC stages are entangled in time-bin observable. BS: beam splitter, SPDC: spontaneous parametric down conversion. 
The pump interferometer is also known as the qubit preparation stage. Therefore, a qubit whose state reads

$$
|\psi\rangle=\frac{1}{\sqrt{2}}\left(|1,0\rangle_{p}+e^{i \varphi}|0,1\rangle_{p}\right),
$$

is found at the first output of the pump interferometer. Conversely, the qubit state at the second output reads

$$
|\psi\rangle^{\prime}=\frac{1}{\sqrt{2}}\left(|1,0\rangle_{p}-e^{i \varphi}|0,1\rangle_{p}\right)
$$

as an additional phase of $\frac{\pi}{2}$ is acquired upon reflection of the output beam splitter (BS2). This explains the orthogonality between the qubit states $|\psi\rangle$ and $|\psi\rangle^{\prime}$. Here, the state $|1,0\rangle_{p}$ describes pump photons, $p$, to be designated in the first time-bin, passing through the shorter arm of the interferometer. In contrast, pump photons that propagate in the opposite long arm, are slotted in the second time-bin, and represented by the state $|0,1\rangle_{p}$.

Both prepared qubits, $|\psi\rangle$ and $|\psi\rangle^{\prime}$, are now subjected to be transformed into entangled time-bin qubits by sending them to two SPDC stages. The creation time of photon pairs, bounded by non-linear interaction in one SPDC stage, is given by the superposition of two values:

$$
|\phi\rangle=\frac{1}{\sqrt{2}}\left(|1,0\rangle_{a}|1,0\rangle_{b}+e^{i \varphi}|0,1\rangle_{a}|0,1\rangle_{b}\right),
$$

where photons $a$ and $b$ are time-bin entangled. Similarly, photons $c$ and $d$, generated by the other SPDC stage are also entangled,

$$
|\phi\rangle^{\prime}=\frac{1}{\sqrt{2}}\left(|1,0\rangle_{c}|1,0\rangle_{d}-e^{i \varphi}|0,1\rangle_{c}|0,1\rangle_{d}\right)
$$

Again, it is important to stress that, due to the phase acquired at the beam splitter in the pump interferometer, the state $|\phi\rangle$ is generated in one of the SPDC stages and the state $|\phi\rangle^{\prime}$ in the other one.

As the origin of the two photon pairs are independent, and prior to this, no form of entanglement exist between either photon $a$ or $b$, with photon $c$ or $d$, the product of these two states, $|\phi\rangle_{a b} \otimes|\phi\rangle^{\prime}=|\phi\rangle_{a b c d}$, can be expressed as

$$
|\phi\rangle_{a b c d}=\frac{1}{\sqrt{2}}\left(|1,0\rangle_{a}|1,0\rangle_{b}+e^{i \varphi}|0,1\rangle_{a}|0,1\rangle_{a}\right) \otimes \frac{1}{\sqrt{2}}\left(|1,0\rangle_{a}|1,0\rangle_{b}-e^{i \varphi}|0,1\rangle_{a}|0,1\rangle_{a}\right)
$$


By rewriting the computational basis in the Bell-state basis:

$$
\left\{\begin{aligned}
|0\rangle|0\rangle & =\frac{1}{\sqrt{2}}\left(\left|\Phi^{+}\right\rangle+\left|\Phi^{-}\right\rangle\right), \\
|0\rangle|1\rangle & =\frac{1}{\sqrt{2}}\left(\left|\Psi^{+}\right\rangle+\left|\Psi^{-}\right\rangle\right), \\
|1\rangle|0\rangle & =\frac{1}{\sqrt{2}}\left(\left|\Psi^{+}\right\rangle-\left|\Psi^{-}\right\rangle\right), \\
|1\rangle|1\rangle & =\frac{1}{\sqrt{2}}\left(\left|\Phi^{+}\right\rangle-\left|\Phi^{-}\right\rangle\right)
\end{aligned}\right.
$$

Then, the four Bell states can be introduced in the quadripartite state $|\phi\rangle_{a b c d}$ such that

$$
\begin{aligned}
|\phi\rangle_{a b c d}= & \left(\left|\Phi^{+}\right\rangle_{b c} \frac{1}{\sqrt{2}}\left(|1,0\rangle_{a}|1,0\rangle_{d}-e^{i 2 \varphi}|0,1\rangle_{a}|0,1\rangle_{d}\right)+\right. \\
& \left|\Phi^{-}\right\rangle_{b c} \frac{1}{\sqrt{2}}\left(|1,0\rangle_{a}|1,0\rangle_{d}+e^{i 2 \varphi}|0,1\rangle_{a}|0,1\rangle_{d}\right)+ \\
& \left|\Psi^{+}\right\rangle_{b c} \frac{1}{\sqrt{2}}\left(e^{i \varphi}|0,1\rangle_{a}|1,0\rangle_{d}-e^{i \varphi}|1,0\rangle_{a}|0,1\rangle_{d}\right)+ \\
& \left.\left|\Psi^{-}\right\rangle_{b c} \frac{1}{\sqrt{2}}\left(e^{i \varphi}|0,1\rangle_{a}|1,0\rangle_{d}-e^{i \varphi}|1,0\rangle_{a}|0,1\rangle_{d}\right)\right) \\
= & \frac{1}{2}\left(\left|\Phi^{+}\right\rangle_{b c}\left|\Phi^{-}(2 \varphi)\right\rangle_{a d}+\left|\Phi^{-}\right\rangle_{b c}\left|\Phi^{+}(2 \varphi)\right\rangle_{a d}+\right. \\
& \left.\left|\Psi^{+}\right\rangle_{b c} e^{i \varphi}\left|\Psi^{-}\right\rangle_{a d}+\left|\Psi^{-}\right\rangle_{b c} e^{i \varphi}\left|\Psi^{+}\right\rangle_{a d}\right)
\end{aligned}
$$

As for now, the most important requirement to perform entanglement swapping is fulfilled. Two time-bin entangled photon pairs, or EPR pairs, are created. The all four corresponding photons, which are properly labeled $a, b, c$ and $d$, will be distributed accordingly. Both photons $a$ and $b$ are destined for Alice and Bob, respectively. As for the rest, photons $b$ and $c$ will be directed towards the Bell-state measurement apparatus.

The next step consists of performing the so-called Bell state measurement (BSM) between the inner photons $b$ and $c$. Before we proceed further, it is important to understand the interpretation conveyed by the Eq.(9.7). BSM permits projecting photons $b$ and $c$ onto one of the four maximally entangled Bell states $\left(\left|\Phi^{+}\right\rangle_{b c},\left|\Phi^{-}\right\rangle_{b c},\left|\Psi^{+}\right\rangle_{b c},\left|\Psi^{-}\right\rangle_{b c}\right)$ and these four possible outcomes are bounded by an equal probability of $\frac{1}{4}$. Moreover, these outcomes will also determine the entangled state projection for the remaining outer photons $a$ and $d$. For instance, an outcome of $\left|\Psi^{-}\right\rangle_{b c}$ causes photons $a$ and $d$ belonging to Alice and Bob, respectively, collapse onto entangled state $\left|\Psi^{+}\right\rangle_{b c}$. This is also true for the other three possible outcomes of BSM. 
Eq.(9.7) also shows that the level of complexity concerning experimental realization could be drastically reduced if photons $b$ and $c$ are projected onto the state $\left|\Psi^{-}\right\rangle_{b c}$, thus having the phase $\varphi$ of the pump interferometer only as a global phase. In this scenario, the photon pair creation process is completely independent to any drift in term of the wavelength of the pump laser, as well as the phase fluctuations inside the pump interferometer. Furthermore, in order to verify whether the entanglement swapping is taking place or not, we should analyze the correlation between photons $a$ and $d$ in term of time-bin entanglement. Yet the entanglement swapping can be done, if and only if, each source produces one photon pair. The most feasible approach to be able to select such cases, is to condition the time-bin correlation of photons $a$ and $d$ on the coincidences between the two inner detectors employed in the BSM system. In this case, only the projection of photons $b$ and $c$ onto the state $\left|\Psi^{-}\right\rangle_{b c}$ will result in a coincidence between the inner detectors due to fermionic nature governing this state thus allowing post-selection type procedure.

Upon successful projection of the state $\left|\Psi^{-}\right\rangle_{b c}$ at BSM, Both Alice and Bob share entangled photons in the state

$$
\left|\Psi^{+}\right\rangle_{a d}=\frac{1}{\sqrt{2}}\left(|0,1\rangle_{a}|1,0\rangle_{d}+|1,0\rangle_{a}|0,1\rangle_{d}\right)
$$

\subsection{The quantum relay dilemma}

BSM lies at the very heart of a quantum relay architecture and a linear optics implementation relies on two-photon interference (see Sec.9.3.2). In order to observe this unique quantum phenomenon, interfering photons at BSM need to be perfectly indistinguishable in every degrees of freedom. This specific point of view, at this stage, one surely should not be able the to tell the difference between the incoming photons by their arrival time. This represents the biggest challenge so far towards achieving a fully operational quantum relay for long distance quantum communication.

Consequently, the very strong constraint on the synchronization between separate entangled photon pair sources needs to be addressed. In particular, any timing uncertainty during the process of creating or detecting the interfering photons, has to be smaller than their coherence time. In this regard, this represent the ultimatum of perfect synchronization [De 
Riedmatten et al. 2005, Yang et al. 2006, Halder et al. 2007, Yang et al. 2009, Kaltenbaek et al. 2009, Takesue \& Miquel 2009, Aboussouan et al. 2010, McMillan et al. 2013].

Being a more conventional and straightforward approach, pulsed operational regimes are adopted in quantum relay architecture in order to comfortably control the creation time of the entangled photon pairs, associated with optical path length matching procedures so as to ensure perfect temporal overlap of the interfering photons at the BSM apparatus. Using ultra-short, femtosecond pump pulses is a well known strategy to reduce the time uncertainty on entangled photon creation process. More interestingly, combining this strategy with broadband filtering for the interfering photons (up to $1 \mathrm{~nm}$ bandwidth), one is capable of removing any residual time uncertainty. This really highlights the advantages of the pulsed regime. In [Kaltenbaek et al. 2009], the above strategy was implemented in a Master-Slave configuration, where two pulsed lasers, one master and one slave, are employed to pump their respective entangled photon pair sources (see also [Kaltenbaek et al. 2006]). In this case, the slave is triggered by the master. This technique relies on the complex implementation of sophisticated phase-locked loops where additional electro $\rightleftharpoons$ optical conversion stages are required, thus introducing unwanted timing jitters to the photon pairs creation time. Consequently, quantum relay link would be limited to distance below $30 \mathrm{~km}$.

Apart from that, especially for long distance applications, another cumbersome challenge would be the necessity of stabilizing the length of the employed optical fiber, within the coherence length of the photons, which is extremely hard to achieve. Previously demonstrated entanglement swapping in femtosecond pulsed regime, either based on polarization entanglement [Pan et al. 1998, Yang et al. 2006, Kaltenbaek et al. 2009, Jin et al. 2015] or time-bin entanglement [de Riedmatten et al. 2003, De Riedmatten et al. 2005, Takesue \& Miquel 2009], show a limitation in terms of achievable distance below $30 \mathrm{~km}$, not to mention the laser repetition rate limitation below $500 \mathrm{MHz}$ due to basic pump laser cavity construction.

Another approach is to opt for continuous wave (CW) regime where entangled photon pairs are randomly, and continuously created. By doing so, the synchronization constraint between two remote sources is completely relaxed. This way, both sources are considered to be completely autonomous. The post-selection of BSM events showing perfect overlap of indistinguishable photons, are accomplished by means of detection. In much simpler words, 
it is now the employed single photon detectors which carry the burden to discriminate such events (see also [Aboussouan et al. 2010]). In this case, provided the absence of any time-binning possibility due to continuous working regime of the pump lasers, the time uncertainty is solely contributed by the timing jitter of the detectors. In CW regime, possible arrival of multiple photons with extremely short coherence time is hidden within the timing jitters of the detectors. To date, designing detectors with such extremely low jitter, in the order of tens of femtoseconds, still remains uncertain due to technological constraints. One of the possible solution is to use ultra narrow band filters (on the order of $10 \mathrm{pm}$ ) which greatly increase the photons' coherence time up to $350 \mathrm{ps}$, combined with low jitter detectors such as superconducting single photon detector (SSPD) [Halder et al. 2007, Halder et al. 2008]. The main concern is that any small drifts in the central wavelength of the mentioned filters greatly affects the quality of indistinguishability in terms of spectral mode. In addition, the extremely small acceptance bandwidth of the filters also reduces the overall experimental rates.

\subsection{Our approach: all-optical synchronization}

In Nice, we proposed an original scheme which is conceptually less complex, inspired by the latest technological advances offered by the telecom industry and non-linear optics as enabling technologies. With this scheme, we aim at demonstrating an all-optical, ultrafast, and picosecond regime approach towards accurate synchronization of remote entangled photon pair sources within quantum relay architecture at long distances. Additionally, our scheme can be done in a fully guided-wave manner ${ }^{1}$.

\subsubsection{Single, master optical clock operation}

The principle of the new proposed scheme is illustrated in Fig.9.2. Within this framework, we emphasize the use of an ultra-fast, state-of-the-art telecom laser at $1540 \mathrm{~nm}$, capable of operating at high repetition rate (from $1 \mathrm{GHz}$ up to $10 \mathrm{GHz}$ ), and emitting picosecond pulses. This laser serves as common master optical clock to "trigger" our two entangled photon pair sources. The wavelength of $1540 \mathrm{~nm}$ is opted to be the operating wavelength

\footnotetext{
${ }^{1}$ Patent pending.
} 
to ensure long range propagation of light in low-loss telecom optical fibers. On the other hand, the very high efficiency of periodically poled lithium niobate waveguides (PPLN/W) has led to the investigation of sequential nonlinear processes. In such a configuration, nonlinear interaction of second harmonic generation (SHG) up-converts the incident light pulses from telecom to visible wavelength, and simultaneously, up-converted light serves as pump pulses for the subsequent SPDC process, creating photon pairs back in the telecom range of wavelengths.

Inspired by such concept, the optical clock pulses produced by the ultra-fast laser not only serve as a trigger for time-binning, but simultaneously act as essential pump field needed by the SPDC to give birth to the photon pairs. The very same approach has been adopted in the development of our ultra-fast heralded single photon source (UFHSPS, see Chapter 8). In other words, the layout of our scheme is no more than a combination of two UFHSPS. The fact that these optical pulses lie in the telecom wavelength range allows unprecedented separation between two remote sources as they propagate over long distances in current telecom optical fibers $(\sim 100 \mathrm{~km})$. The next phase consists of separation and distribution of the photon pairs in a fully guided-wave fashion for appropriate Bell-state measurement and detection. Up to this level, the separation between our two photon pair sources is about $200 \mathrm{~km}$ and it is estimated that when the implementation is finalized, the distance separating the two communicating parties, Alice and Bob, can be increased up to $400 \mathrm{~km}$.

Speaking of the advantage of our scheme, the notion of "all-optical" approach ensures high timing accuracy whereas, compared to others, our configuration consists only of one telecom laser as master optical clock, avoiding any necessity for opto-electrical conversion stages. The only conversion that takes place is the up conversion of optical master clock pulses from $1540 \mathrm{~nm}$ to $770 \mathrm{~nm}$ in which any small timing uncertainty of optical conversion process is completely negligible, i.e. essentially jitter-free environment. Additionally, operating in the picosecond regime allows us to exploit off-the-shelves, high-performance, low-loss and inexpensive telecom devices for photon filtering namely the DWDM and Bragg gratings filters. Apart from that, the path length (a few $\mathrm{mm}$ ) and also the central wavelength of the employed filters can be easily stabilized using simple and off-the-shelves PID controllers [Takesue \& Miquel 2009, Aboussouan et al. 2010]. 


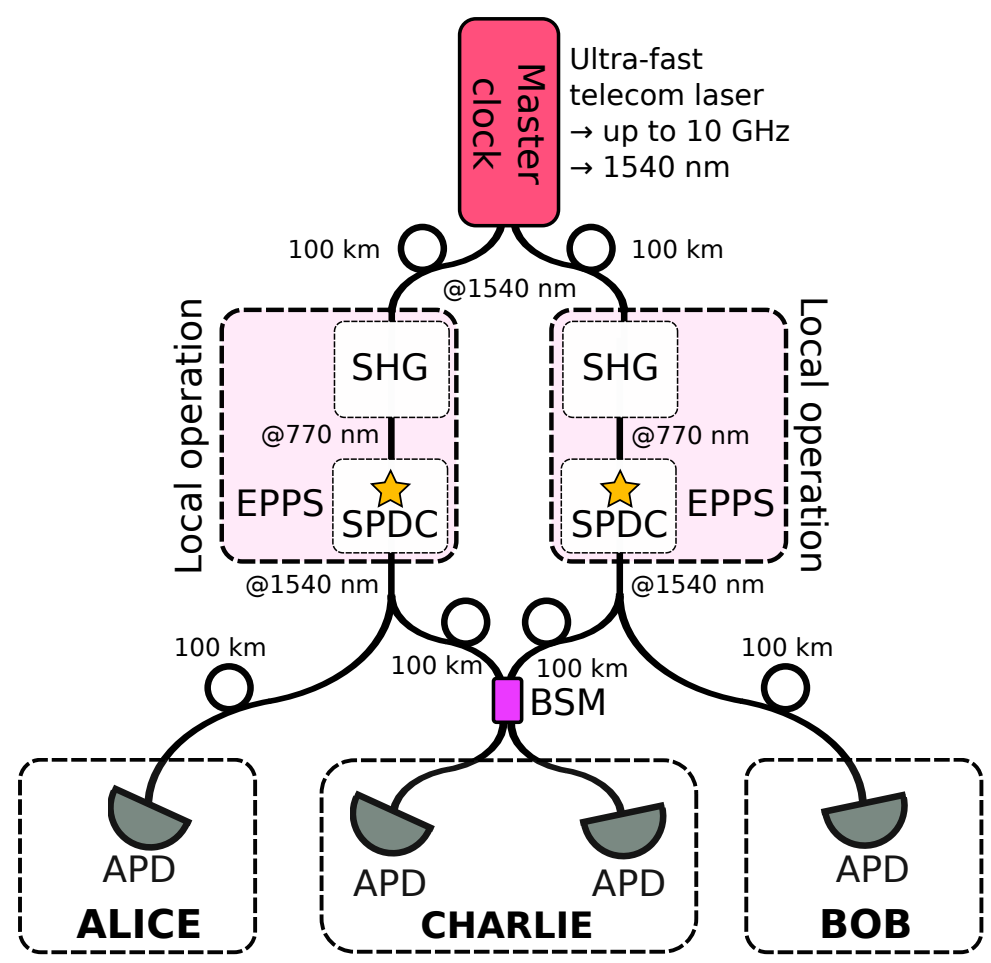

Figure 9.2: Outline of the proposed all-optical synchronization scheme. SHG (second harmonic generation block), EPPS (entangled photon pair source), BSM (Bell-state measurement), APD (avalanche photodiode).

Even though photons in the telecom range of wavelengths experience less absorption in existing deployed optical fibers (typical loss of around $0.2 \mathrm{~dB} / \mathrm{km}$ ), this eventually will become one of the major limiting factor as we are trying to push the quantum communication channels beyond hundreds of kilometers. To overcome this problem, it is vital to increase the throughput capacity of the SPDC process for photon pair generation, so as to be able to operate always above the intrinsic dark counts level of the employed single photon detectors at the end of the channel, while maintaining the mean number of created paired photon per mode (temporal, spectral, and spatial) to a level that guarantees high-quality entanglement. In the case of quantum relay implementation, researchers often try to find a good compromise, i.e. trade-off between the maximum achievable count rates and the best entanglement visibility. In most cases, higher count rates are achieved by increasing the pump power resulting in poor entanglement quality due to multi-pair contributions polluting the experiment [Herbst et al. 2014], as well as rendering potentially implemented QKD protocols less secure. 
Our strategy of integrating the UFHSPS (at a lower speed) demonstrated in Chapter 8 of this manuscript can guarantee high success rates for any subsequent protocols while maintaining the multi-pair contributions to the minimum, as well as ensuring the optimum entanglement visibility. Underlying is the idea of "filling the empty spaces" in the temporal domain.

\subsubsection{Hong-Ou-Mandel interference for validation}

On the contrary to $\mathrm{CW}$ regime, the intrinsic time-binning of the photons induced by the pulse regime operation allows the perfect control of not only the creation time of entangled photon pairs by suitable nonlinear crystals, but also the simultaneous arrival time of two identical photons at a 50/50 beam splitter as required by the Bell state measurement. A feasible way to verify the latter is to take advantage of the so-called quantum effect of two-photon interference, i.e. photon coalescence [Hong et al. 1987, Rarity \& Tapster 1989, Rarity 1995, Legero et al. 2003, Halder et al. 2005, Aboussouan et al. 2010]. Thus, successful observation of such quantum interference serves as a validation tool for an operational quantum relay architecture.

Consider two photons are sent into two different inputs of a 50/50 beam splitter. Together, upon arrival at the beam splitter, if these two photons cannot be distinguished in any degree of freedom, namely the spatial mode, spectral mode, polarization mode and time of arrival, a quantum interference effect will occur. To quantify and observe such effect, the ideal and practical approach would be the scanning of the arrival time of one of the photons at the beam splitter. With the help of two single photon detectors connected to both outputs of the beam splitter, a dipping signature will be revealed during the acquisition of coincidence events due to the fact that both photons, provided they are indistinguishable, exit the beam splitter together through the same output. This absence of coincidences in the form of a dip is known as Hong-Ou-Mandel (HOM) dip [Hong et al. 1987].

\subsubsection{Quantum two-photon interference model}

The modeling of two-photon interference effect begins with describing the input number state, or Fock state, of two photons sent to a 50/50 beam splitter through two different 


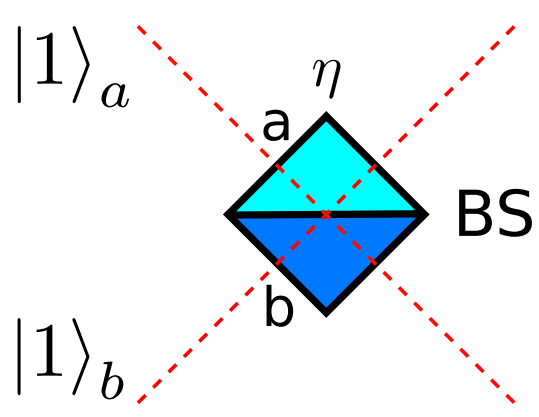

Figure 9.3: Representation of two photons impinging on a beam splitter, with their corresponding states are described. BS (beam splitter), $\eta$ (reflectivity of the beam splitter).

input modes (see Fig.9.3);

$$
\left|\Psi_{i n}\right\rangle=|1\rangle_{a}|1\rangle_{b}=\hat{a}^{\dagger} \hat{b}^{\dagger}|0\rangle
$$

where $a$ and $b$ represent two different input mode of the beam splitter and both modes carry the creation operators $\hat{a}^{\dagger}$ and $\hat{b}^{\dagger}$, respectively. In the following, we consider these two photons are perfectly indistinguishable, in term of spatial, spectral, and polarization modes, as well as in term of temporal overlap within their coherence time. Being a unitary transformation, the effect of such a beam splitter with certain reflectivity $\eta$ on the operators leads to

$$
\hat{a}^{\dagger} \stackrel{B S}{\longrightarrow} i \sqrt{\eta} \hat{a}^{\dagger}+\sqrt{1-\eta} \hat{b}^{\dagger}
$$

and

$$
\hat{b}^{\dagger} \stackrel{B S}{\longrightarrow} \sqrt{1-\eta} \hat{a}^{\dagger}+i \sqrt{\eta} \hat{b}^{\dagger}
$$

where the pure imaginary complex number $i$ denotes a $\frac{\pi}{2}$ phase acquired each time a photon is reflected on the beam splitter. Eventually, we obtain the output state

$$
\begin{aligned}
\left|\Psi_{\text {out }}\right\rangle & =\left(i \sqrt{\eta} \hat{a}^{\dagger}+\sqrt{1-\eta} \hat{b}^{\dagger}\right)\left(\sqrt{1-\eta} \hat{a}^{\dagger}+i \sqrt{\eta} \hat{b}^{\dagger}\right)|0\rangle \\
& =\left(i \sqrt{\eta} \sqrt{1-\eta} \hat{a}^{\dagger 2}+(1-\eta) \hat{a}^{\dagger} \hat{b}^{\dagger}-\eta \hat{a}^{\dagger} \hat{b}^{\dagger}+i \sqrt{\eta} \sqrt{1-\eta} \hat{a}^{\dagger 2}\right)|0\rangle
\end{aligned}
$$

after the beam splitter. In the case where the beam splitter exhibits a reflectivity of $\frac{1}{2}$, Eq.(9.12) can be further simplified to

$$
\left|\Psi_{\text {out }}\right\rangle=\frac{1}{2}\left(i \hat{a}^{\dagger 2}+i \hat{b}^{\dagger 2}\right)|0\rangle=\frac{i}{\sqrt{2}}(|2,0\rangle+|0,2\rangle) .
$$


The final state of Eq.(9.13) is famously know as the N00N state, simply because upon exiting the beam splitter, the two photons can always be found together at one of the outputs in a random manner. This is due to the destructive interference concerning the case where the two photons exit the beam splitter at different output.

\subsubsection{Visibility tuning by adjusting the temporal overlap}

The control of the indistibuishability of two photons plays an important role on determining the quality, i.e. visibility, of the two-photon interference effect. The level of indistinguishability becomes an important parameter that can be adjusted by experimentalists in order to achieve the best interference visibility possible. As synchronization is at the heart of our approach, in this section, we are interested solely in the study of achievable visibility characterized by the temporal overlap of two photons impinging on a 50/50 beam splitter, provided the other degrees of freedom are perfectly under control.

Consider photons that share the same temporal distribution, whose the Gaussian temporal function is defined by

$$
f(t)=\frac{1}{\sqrt{2 \pi} \sigma_{\tau_{c}}} e^{-\frac{t^{2}}{2 \sigma_{\tau_{c}}^{2}}},
$$

with $\sigma_{\tau_{c}}$ representing the standard deviation of a Gaussian distribution related to the coherence time $\tau_{c}$ of the photons. By introducing a relative time difference $\delta t$ on the arrival times of the two photons at the 50/50 beam splitter, the corresponding probability of having an interference is given by

$$
\Gamma(\delta t)=\frac{(f(t) * f(t))(\delta t)}{\int_{-\infty}^{+\infty} d t f(t) f(t)}=e^{-\frac{\delta t}{4 \sigma_{\tau_{c}}^{2}}} .
$$

A straightforward calculation shows that when imposing $\delta t=0, \Gamma(0)=1$ is obtained, corresponding to $100 \%$ probability of obtaining an interference. So far, we assume no time incertitude influencing the temporal overlap of both photons. When this time incertitude is taken into account, the new probability of observing an interference can be expressed as $\Gamma(\delta t+\Delta t)$. We will assume that the time fluctuations $\Delta t$ are gaussian distributed around $\Delta t=0$ with a deviation $\sigma_{\tau_{e} r r}$, and follow the function:

$$
f_{e r r}(\Delta t)=\frac{1}{\sqrt{2 \pi} \sigma_{\tau_{e r r}}} e^{-\frac{(\Delta t)^{2}}{2 \sigma_{\tau}^{2}{ }_{e r r}}}
$$


When averaging the function $\Gamma(\delta t)$ on the different $\Delta t$ contributions, we obtain

$$
\langle\Gamma(\delta t)\rangle=\int_{-\infty}^{+\infty} d \Delta t \Gamma(\delta t+\Delta t) f_{e r r}(\Delta t)=\frac{1}{\sqrt{\frac{\sigma_{\tau_{e r r}}^{2}}{2 \sigma_{\tau_{C}}^{2}}+1}} e^{-\frac{\delta t^{2}}{4 \sigma_{\tau_{C}}^{2}\left(1+\rho^{2}\right)}}
$$

On the contrary to the ideal case, here, a $\delta t=0$ leads to a maximum visibility of

$$
V_{e r r}^{\max }=\frac{1}{\sqrt{\frac{\sigma_{\tau_{e r r}}^{2}}{2 \sigma_{\tau_{c}}}+1}}=\frac{1}{\sqrt{\rho^{2}+1}} \leq 1 \text {, with } \rho^{2}=\frac{\sigma_{\tau_{e r r}}^{2}}{2 \sigma_{\tau_{c}}^{2}} .
$$

Eq.(9.18) clearly states that a near $100 \%$ interference visibility can be obtained by approaching the parameter $\rho^{2}$ to zero. This condition of $\sigma_{c}^{\tau} \gg \sigma_{\text {err }}^{\tau}$ emphasizes the requirement of manipulating photons showing a larger coherence time respect to any time uncertainty. Eventually, we note that, based on Eq.(9.17), we expect to achieve a greater visibility with lower standard deviation for smaller $\rho$ as illustrated in Fig.9.4.

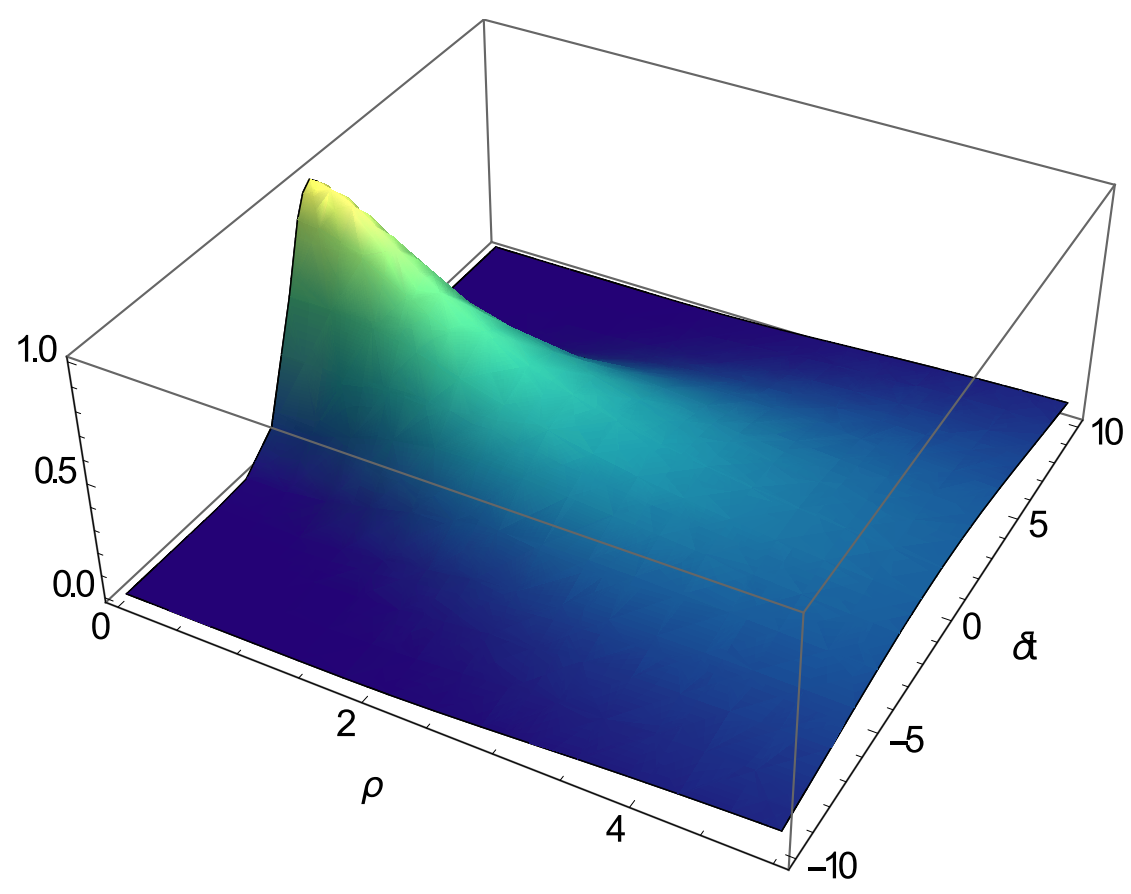

Figure 9.4: Achievable visibility under hypotheses of Eq.(9.17) as a function of $\rho$ and $\delta t$. 


\subsection{Preliminary tests}

In order to check the quality of our setup, we run a preliminary two-photon interference experiment in a quantum relay configuration where the interfering photons are originated from two independent sources. Through the HOM dip experiment, certain important aspects such as the generation of photon pairs through SPDC process, spectral tailoring by means of filtering, the quality of indistinguishability of interfering photons and overall photon detection scheme are validated. The overall experimental apparatus for preliminary test is represented in Fig.9.5. Here, we momentarily use a standard solid-state laser (Coherent MIRA 900-D) which simulates the combined ultra-fast master clock laser together with the SHG stage to produce SPDC pump photons.

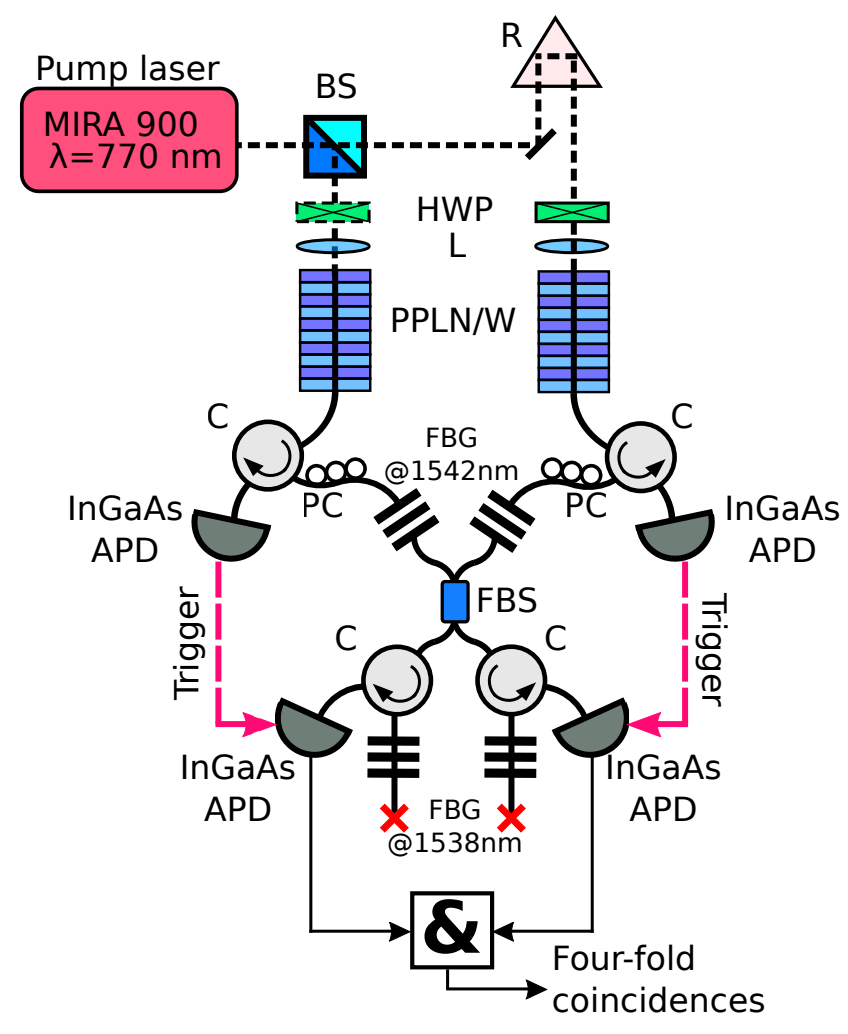

Figure 9.5: Momentary experimental apparatus for the HOM dip experiment. BS (beam splitter), R (motorized retroreflector), HWP (half wave plate), L (lens), PPLN/W (periodically poled lithium niobate waveguide), C (optical circulator), PC (polarization controller), FBG (fiber Bragg gratings filter), FBS (fiber beam splitter), APD (avalanche photodiode), \& (4-fold coincidence counter).

Apart from the repetition rate, the Coherent MIRA 900 laser exhibits the same spectral 
characteristics as the visible light generated by the intended SHG stage in the original proposed scheme (see also Sec.8.2.1 for Chapter 8): the laser operates at the wavelength of $770 \mathrm{~nm}$, providing $2.2 \mathrm{ps}$ temporal duration pulses at repetition rate of $76 \mathrm{MHz}$. Using a suitable arrangement of standard optical components, the beam is then divided into two paths towards two SPDC stages implemented each in a $2 \mathrm{~cm}$ long, home-made type0 PPLN waveguide (the same as used in UFHSPS implementation of Chapter 8) which generates wavelength degenerate photon pairs at $1540 \mathrm{~nm}$. The waveguide structures of the nonlinear crystals provide high SPDC efficiencies due to high confinement of light along the waveguide. Due to phase matching conditions, the temperature for both SPDC stages is carefully tuned and stabilized at around $101^{\circ} \mathrm{C}$ by using commercially available temperature controllers (THORLABS TEC 200). As shown in Fig.9.5, just before the SPDC stage on the right, the pump beam at $770 \mathrm{~nm}$ passes through a motorized retro-reflector.

Next, the produced down converted photons pairs at each output of both SPDC stages are collected with single mode fibers and the paired photons are deterministically separated thanks to a combination of two fiber Bragg gratings (FBG) filters, ensuring the selected photons meet the Fourier transform criterion. As already discussed, the task of the employed FBG filters is to select corresponding photons in certain region along the broad emission spectrum of SPDC and their center wavelength is carefully chosen so as to satisfy the conservation of energy. The first FBG selects photons at $1542 \mathrm{~nm}$ within a bandwidth of $800 \mathrm{pm}$ (idler photons), meanwhile the other is centered at $1538 \mathrm{~nm}$ within a bandwidth of $250 \mathrm{pm}$ and picks up the corresponding, symmetrical, twin photons at lower wavelength (signal photons). Narrow filtering on signal photons provides some degrees of relaxation for the time accuracy in the interference experiment. Moreover, broader filtering of idler photons allows respect the energy conservation in broadband regime. Here, the detection of idler photons using two InGaAs APDs (IDQ 210) herald the presence of signal photons in the experimental setup. The signal photons from each source are then directed towards a 50/50 fiber beam splitter for the two photon interference at the output of which are put another two InGaAs-APDs (IDQ 201) connected to an electronic AND gate for the coincidence measurement. We stress that, as these APDs are gated by the signals coming from idler photon detections, the output of the AND gate gives 4-fold coincidence events. Prior to observing the so-called HOM dip phenomena in a two-photon interference experiment, the 
indistinguishability of the signal photons with respect to the spatial, spectral, and the polarization modes need to be validated. The spatial mode matching is guaranteed by the use of single mode fibers to collect the down converted photons exiting both SPDC stages. Identical FBGs at $1538 \mathrm{~nm}$ ensure that signal photons exhibit the same spectral properties. Polarization indistinguishability is verified by using standard polarization controllers and an additional polarizing beam splitter (not represented in Fig.9.5) put at the output of the 50:50 beam splitter and then removed during the coincidence measurement. Once the mentioned degrees of freedom are perfectly controlled, the motorized retro-reflector in one of the pump path is scanned. By doing so, the arrival time of emitted signal photons at one of the inputs of the fiber beam splitter is tuned as required to reveal a dipping signature during the acquisition of 4 -fold coincidences, i.e the HOM dip.

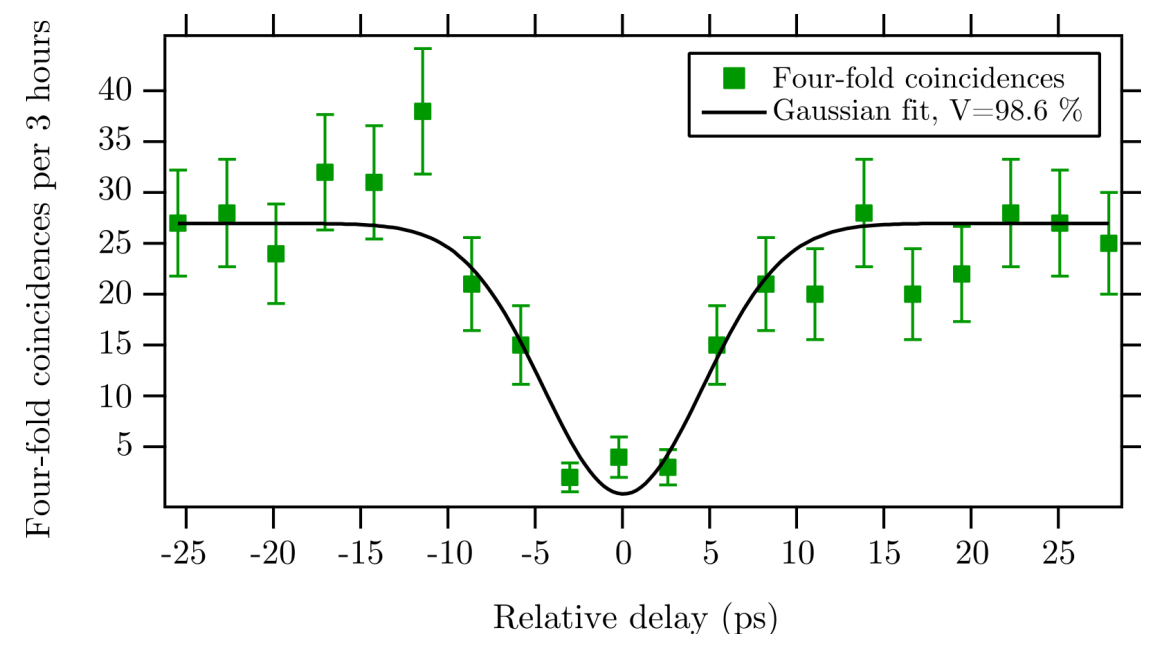

Figure 9.6: The accumulated 4-fold coincidences during 60 hours HOM dip measurement for a total of 20 points. The solid line represents a Gaussian fit to the data. The recorded raw visibility is $98.6 \%$.

The experimental results of the raw 4-fold coincidence rates are represented in Fig.9.6. The total duration of the HOM dip experiment is 60 hours with an integration time of 3 hours for each 20 points. With $1 \mathrm{~mW}$ of pump power supplied to both SPDC stages, the obtained raw visibility of the HOM dip is $98.6 \pm 3 \%$. In addition, the 4 -fold coincidence rates induced only by the dark counts of detectors employed is measured to be negligible. Besides, the measured coherence time directly obtained from the FWHM of the HOM dip is in good agreement with the theoretical value of the coherence time of the corresponding 
photon at $1538 \mathrm{~nm}\left(\tau_{c o h}=14 \mathrm{ps}\right)$ with a Gaussian spectral profile, given by

$$
\tau_{c o h}=0.44 \frac{\lambda^{2}}{c \Delta \lambda} .
$$

Through this preliminary test, we have successfully validated some important aspects of our scheme namely the photon pairs generation through SPDC process at the desired phase matching condition, the photon choice by means of filtering, and the quality of indistinguishability of interfering photons through the HOM dip experiment (see also [Aboussouan et al. 2010]).

\subsection{Towards the first prototype of HOM experiment}

With respect to the preliminary test setup, the following points are highlighted to reflect the important changes that will be introduced in the first prototype for HOM experiment of our scheme (see Fig.9.9).

As operating at much higher repetition rate easily increases the overall rates of the experiment, an ultra-fast picosecond telecom laser at $1540 \mathrm{~nm}$ operated at $2.5 \mathrm{GHz}$ will be employed as master optical clock. Locally, cascaded non-linear frequency conversion (SHG+SPDC) will be exploited so as to comply with the phase matching condition for creating photon pairs in telecom wavelength by the SPDC process in the PPLN/W. As has been mentioned before, this leads to a configuration extremely similar to the one used for the ultra-fast heralded single photon source (UFHSPS, chapter 8). For this particular experiment, the SHG up-conversion stages consist of AR coated, bulk magnesium-doped PPLN (MgO:PPLN) crystals (Covesion). Traditionally, undoped PPLN devices (bulk or waveguide structure) suffer greatly from photorefractive effect [Buse et al. 2007,Kashin et al. 2011] due to their intrinsic properties of large electro-optic coefficient, strong photo-voltaic effect, and strong space charge field. Contrarily to undoped PPLN devices, $5 \%$ of magnesium are added to lithium niobate in order to make the crystal more resistance against such effect, hence higher damage threshold. Additionally, this advantage is achieved while the high nonlinear coefficient of lithium niobate is preserved thus making doped crystals suitable for high power applications. In our experiment, two bulk PPLN samples are used as SHG 
stages. The characterizations of the two periodically poled bulk samples have been carried out. All the characterizations have been done using $2.5 \mathrm{GHz}$ repetition rate laser (Pritel UOC) emitting light at $1540.135 \mathrm{~nm}$ along with an erbium doped fiber amplifier (EDFA). In the following, the characteristic from one sample is presented for simplicity reason. The recorded SHG spectrum is shown in Fig.9.7 while Fig.9.8 describe the evolution of the SHG output intensity as a function of the injected pump power. Note that the two PPLN bulk samples exhibit similar performances.

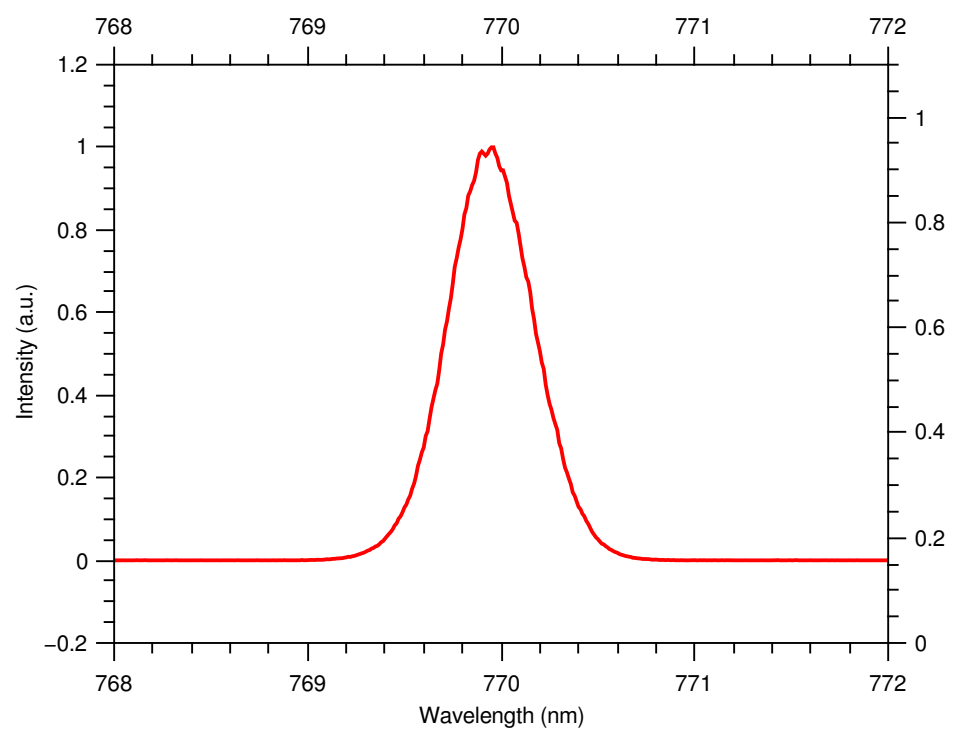

Figure 9.7: Recorded SHG spectrum centered around $770.05 \mathrm{~nm}$ with an FWHM of $0.48 \mathrm{~nm}$. Such a phase matching condition is obtained through the use of periodically poled grating of $18.80 \mu \mathrm{m}$. The sample is temperature stabilized at $111.2^{\circ} \mathrm{C}$.

Further modifications are done to the setup on the choice of photon filtering, i.e. the wavelength of signal (ITU 50, $1538 \mathrm{~nm}$ ) and idler photons (ITU 42, 1543.73 nm). Furthermore, as it will be shown, we replace the 50/50 fiber beam splitter with a combination of two fiber polarization beam splitters (f-PBS) and a polarization controller. This configuration provides a better robustness against the variations of the interfering photon polarizations along the propagation. Moreover, the filtering of signal photons originating from different SPDC sources is performed by a single FBG, so automatically ensuring the indistiguishability in term of spectral mode. In other word, the spectral mode is immune to the shifting wavelength of the FBG. 


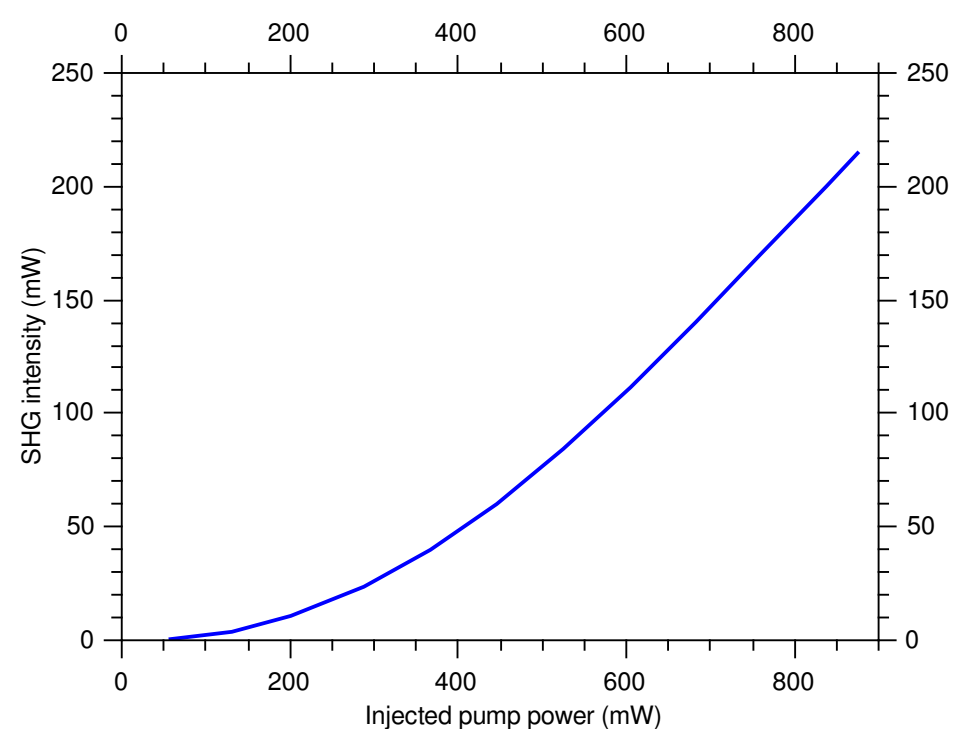

Figure 9.8: Recorded SHG output intensity as a function of the injected pump power (mW).

\subsubsection{Setup for the two photon interference}

In contrast to the preliminary HOM dip experiment discussed in Sec.9.4, and as shown in Fig.9.9, we implement here a different setup for the two-photon interference. In this scheme, the two interfering signal photons are sent to a fiber polarization beam splitter (f-PBS) cleaning their respective polarizations and projecting their original state on the quantum state $|\psi\rangle=|H\rangle|V\rangle$. A polarization controller (PC) is used to turn the polarization state of the pair by $45^{\circ}$, leading to the state:

$$
|\psi\rangle_{P C}=\frac{1}{2}(|H\rangle|V\rangle-|H\rangle|H\rangle+|V\rangle|V\rangle-|V\rangle|H\rangle)
$$

If the two original photons are indistinguishable in any degree of freedom, namely spatial, spectral and temporal modes, except for their polarization upon impinging the f-PBS, the $|H\rangle|V\rangle$ and $|V\rangle|H\rangle$ contributions interfere destructively, therefore reducing the state to $|H\rangle|H\rangle$ and $|V\rangle|V\rangle$ contributions only. As a consequence, when sending the state of Eq.9.20 to a second f-PBS, both photons will always exit through the same output. This is how the indistinguishability between two cross-polarized photons can be quantified by a HOM-type experiment. We stress that, in our case, the filtering of signal photons originating from separate SPDC sources is performed by a single fiber Bragg grating (FBG) filter, placed 


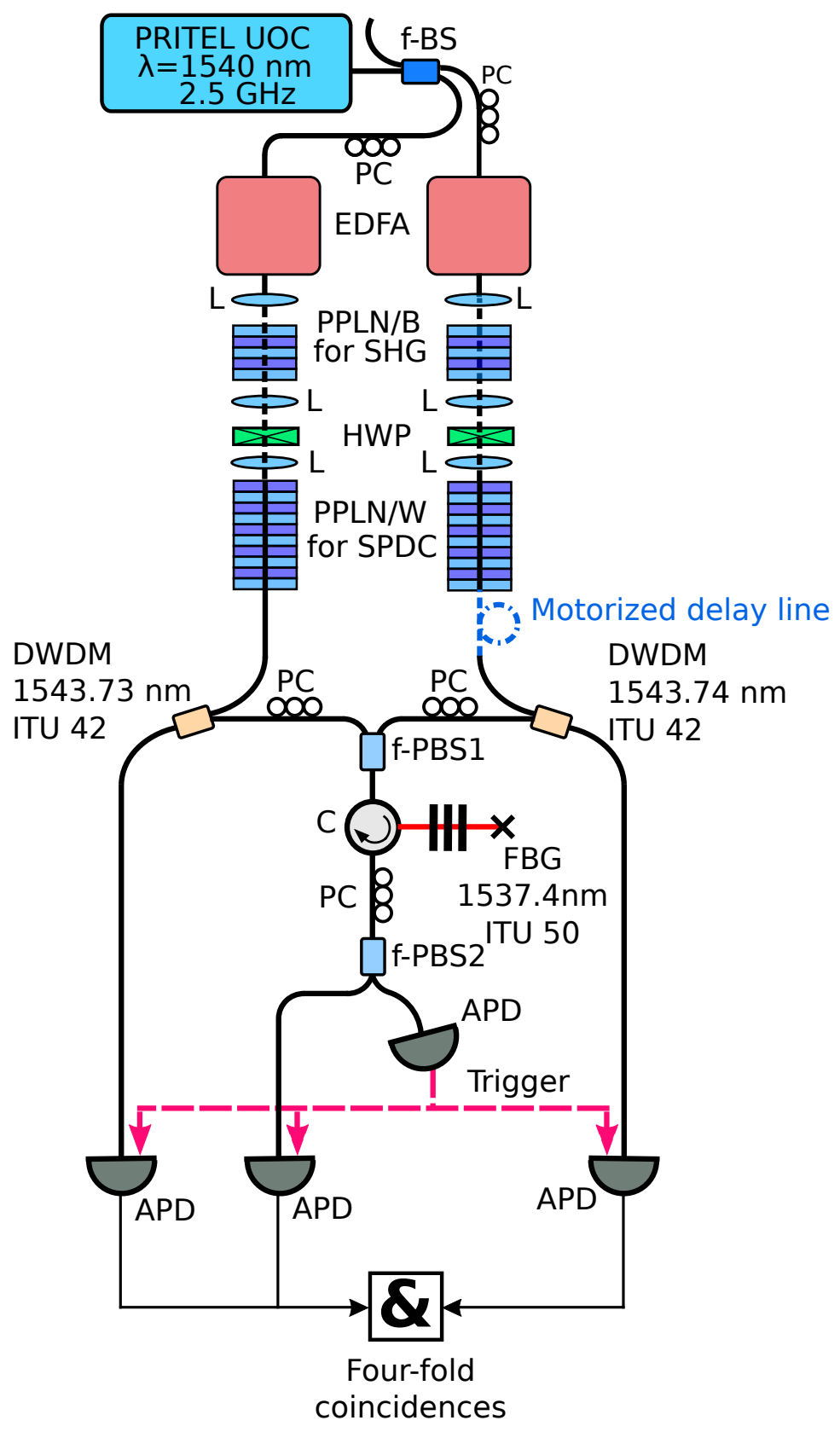

Figure 9.9: Experimental arrangement towards enabling the first prototype of HOM experiment for the validation for our synchronization scheme at $2.5 \mathrm{GHz}$. BS (beam splitter), PC (polarization controller), EDFA (erbium-doped fiber amplifier), L (lens), PPLN/B (PPLN bulk), PPLN/W (PPLN waveguide), HWP (half-wave plate), f-PBS (fiber polarizing beam splitter), DWDM (densed wavelength division multiplexer), C (circulator), FBG (fiber Bragg gratings filter), APD (avalanche photodiode), SSPD (superconducting single photon detector), \& (4-fold coincidence counter). 
right after the first f-PBS. Accordingly, the indistinguishability in term of spectral mode is automatically ensured and it is immune to the shifting wavelength of the FBG filter.

\subsubsection{The path length matching procedure}

Adjusting the length of optical paths along which the interfering photons travel is mandatory for obtaining the condition $\delta t=0$, i.e. for ensuring that, in the HOM dip experiment, the two photons perfectly overlap on the f-PBS in the temporal domain. However, when working with high repetition rate regimes, the path length matching procedure represents one of the most difficult tasks towards observing the HOM dip. For practical reasons, we opted for a path length matching strategy based on the synchronization of classical (bright) optical signals. The synchronization is performed through the detection of pulses obtained by splitting in two, the pulses from an additional, low repetition rate telecom laser that replaces the master clock. This approach brings huge advantage of "universality" with respect to operational repetition rate of the experiment and permits changing on the fly the operational repetition rate of the master clock laser without introducing any further modifications to the optical paths. Note that slow repetition rate regime simplifies the alignment procedure as it allows comfortably discriminating signals originated from two subsequent laser pulses [Aboussouan et al. 2010]. Particularly, we employ an alignment laser emitting femtosecond pulses at the repetition rate of $76 \mathrm{MHz}$ (Calmar Mendocino). The laser exhibits a broad spectral bandwidth of more than $30 \mathrm{~nm}$ which is enough to provide photons corresponding to the central wavelength of all the filters used in our experiment, so as to have, at a given time, single photons propagating in all of the four paths after the SPDC stages. The path length matching procedure is two-fold:

First step Rough alignment of optical path matching based on the observation of the coincidence peak between two single photon detectors.

Second step Fine tuning with the help of classical single photon interference.

FIRST STEP: In Fig.9.11 (a), the ultra-fast $2.5 \mathrm{GHz}$ laser is replaced by the femtosecond Calmar laser. This laser exhibits pulse duration of $100 \mathrm{fs}$, and spectral bandwidth of about $30 \mathrm{~nm}$ centered at $1553 \mathrm{~nm}$ (see Fig.9.10). The pulses are strongly attenuated, down to the single photon regime, and each of them is split into two by a fiber beam splitter. 
Due to waveguide structure of the SPDC stages, two polarization controllers are used to later match the polarization mode to the axis of the crystal. Next, corresponding pulses in each path propagate freely through the EDFAs and the cascaded PPLN stages until finally arriving at the DWDM filters. We stress that low power are employed so as to neglect SHG effects on the Calmar pulses and detector saturation. After the PPLN waveguides, the laser photons conform to the transmission wavelength of the DWDM filter pictured to the left, are detected by APD2.

On the contrary, the rest of the reflected photons from both DWDM filters, are then recombined at f-PBS1 whose transmission is optimized with the help of two polarization controllers. The transmitted photons continue to propagate in the same spatial mode until they are filtered by the FBG. Filtered photons are eventually directed towards f-PBS2 whose output is connected to APD1. Coincidences between the two APDs are visualized in the form of an histogram with the help of a time-to-amplitude converter (TAC) (not represented in Fig.9.11 (a)). In the case where the two optical paths exhibit a certain difference in length (starting from f-BS until f-PBS2), two potentially mismatched coincidence peaks are observed. Depending on which path is longer or shorter, these two coincidence peaks can be easily superposed by introducing optical fiber delays in the bold orange paths (see Fig.9.11 (a)).

SECOND STEP: This step of fine tuning consists of scanning the position of the employed motorized delay line so as to figure out the expected position corresponding to the bottom of the expected HOM dip. Taking advantage of a big Mach-Zehnder-type interferometer configuration (represented by red paths in Fig.9.11 (b)), classical interference can be observed. Here, the optimal interference visibility corresponds to path length matching within the coherence length of the Calmar laser. In this regard, the use of the femtosecond pulsed laser provides better precision to the measurement. A standard power-meter (Coherent Fieldmax) is placed at one of the output of the second f-PBS. Depending on the amount of mismatch, the measured power is $P_{\text {fieldmax }}=\frac{1}{2}(1+\cos \phi)$. By scanning the motorized delay line put right after one of the SPDC stages, the position that gives near $100 \%$ visibility for the observed oscillation is noted to be the expected position to have near $100 \%$ visibility for our future HOM dip.

By scanning the motorized delay line, interference fringes can be observed. The scan- 


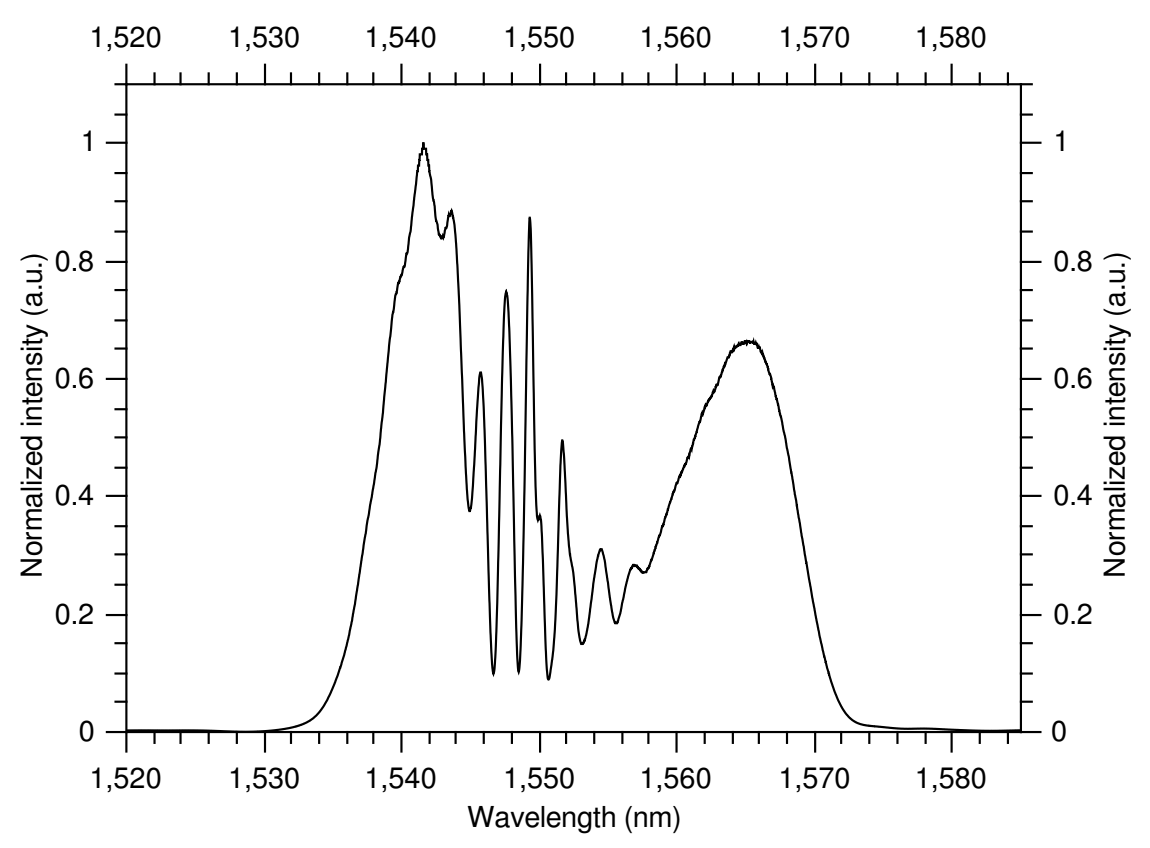

Figure 9.10: Typical emission spectrum of the femtosecond Calmar Mendocino laser.

ning position that gives near $100 \%$ visibility for the observed oscillation is noted to be the expected position to have near $100 \%$ visibility for our future HOM dip.

\subsubsection{Experimental results and discussions}

In this experiment, a free-running avalanche photo-diode (IDQ 220) featuring a quantum detection efficiency of $25 \%, 7 \mu \mathrm{s}$ dead time, and dark count probability of $10^{-6} / \mathrm{ns}$ is employed at one of the f-PBS outputs as heralding detector providing trigger signal towards three other gated APDs (IDQ 201). All the three id201s exhibit 20\% quantum detection efficiency, $7 \mu$ s dead time, and a dark count probability of $10^{-5} / \mathrm{ns}$. To observe the dip behavior, the arrival time of one of the photons on the second f-PBS is tuned by the employed motorized delay line and 4-fold coincidence rate among the signals are registered for each position of the delay line.

The accumulated raw 4-fold coincidences are plotted in Fig.9.12. All data are normalized to the heralding detector rate. In our case, the use of the free-running IDQ 220 as the heralding detector limits the maximum achievable heralding rate. As a matter of fact, indeed, such a detector is only capable of counting photons up to $60 \mathrm{KHz}$. This imposes a 

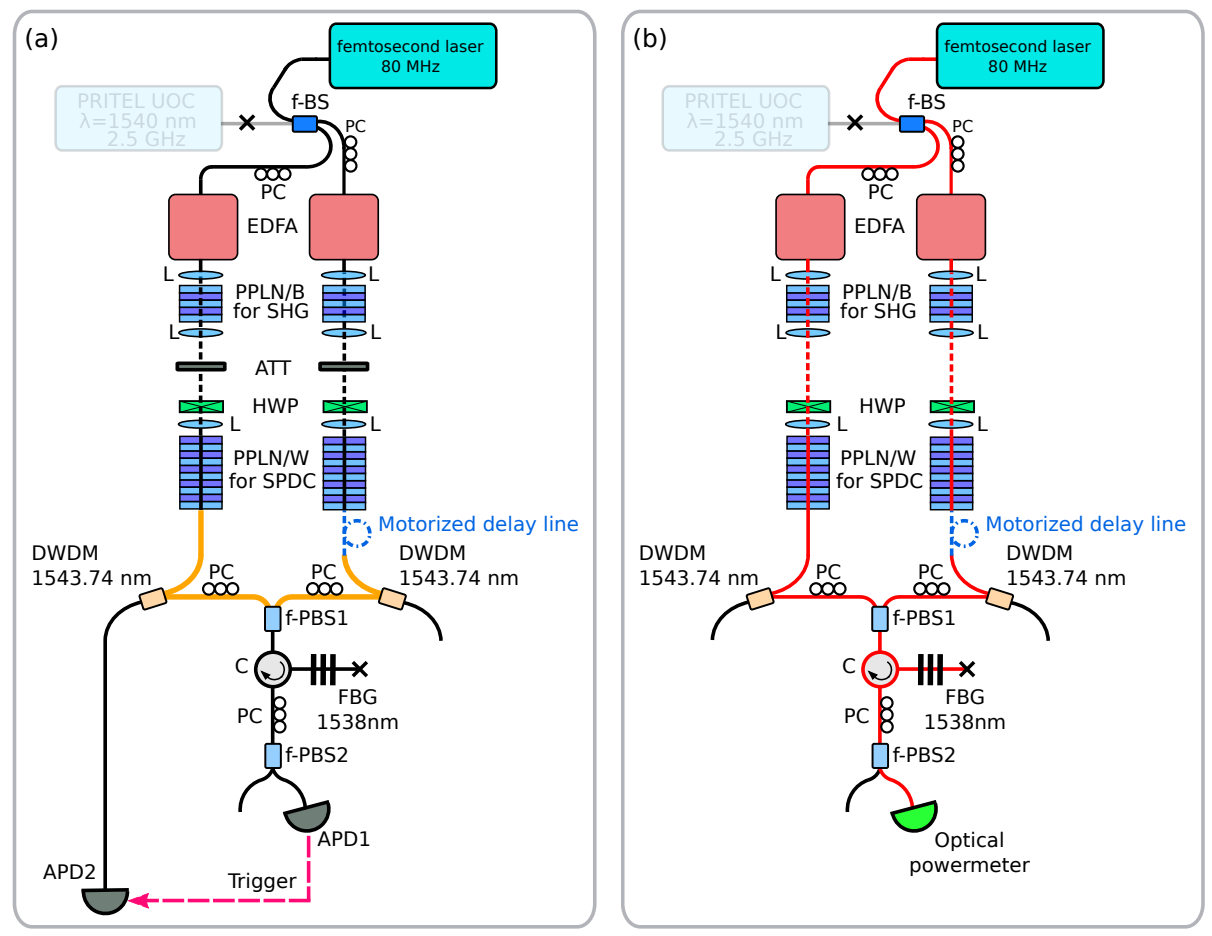

Figure 9.11: The path length matching procedure by using a wideband, femtosecond pulsed laser. (a): Path length difference is roughly matched by observing the coincidence peak registered by two APDs. (b): Fine adjustment of path length matching through observation of classical single photon interference in a unstabilized, big Mach-Zehnder interferometer represented by highlighted red path 
low pumping regime and, as a consequence, a low 4 -fold coincidence events. Due to this, we obtained on average, fifteen 4 -fold coincidences for three hours of integration time. The whole measurement takes a duration of 21 hours with an integration time of 3 hours for each 7 points. Due to the long time acquisition and some instability issues, notably mechanical fluctuations of the translation stages supporting the employed PPLN samples, a progressive reduction in the overall experimental rate is observed.

Let us now emphasize that the obtained HOM dip stands as a preliminary result, associated with encouraging results. First and foremost, the position of the delay line, where the minimum 4-fold coincidence rate is observed, corresponds well to the one found through the path length matching procedure described in Sec.9.5.2. Moreover, the temporal width ( $\sim 13.6 \mathrm{ps}$ ) of the observed dip is in agreement with the coherence time of the photons (14 ps). This strongly indicates that such a measurement outcome is definitely due to HOM-type interference. Last but not least, and despite the normalization of the data we observe a near perfect raw visibility as the 4-fold coincidence counts drop almost to zero at zero delay. Accordingly, the synchronization scheme with a $2.5 \mathrm{GHz}$ repetition rate pulsed laser clock is validated through this particular experiment.

Without doubt, the choice of the heralding detector is important in order to unleash the true potential of our proposed synchronization scheme. Measurement are currently ongoing with a new detection scheme able to handle much higher detection rate without being limited by saturation effects.

\subsubsection{Conclusions and perspectives}

In this chapter, the application of ultra-fast telecom technology, notably for quantum relay application, is proposed. In this framework, The scheme relies on the use of ultra-fast master-clock optical pulses delivered by a $2.5 \mathrm{GHz}$ repetition rate telecom laser, which then serves as pump for entangled paired photons generation in separate sources. The distribution of such optical clocks at telecom wavelength is done in a fully guided wave fashion, thus allowing unprecedented separation between two remote sources. Moreover, such ultra-fast pulsed laser is useful in fighting low success rates of quantum protocols due to probabilistic photon pair generation via SPDC process based on non-linear crystals. Also note that our synchronization technique offers great simplicity to the experimental setup compared to pre- 


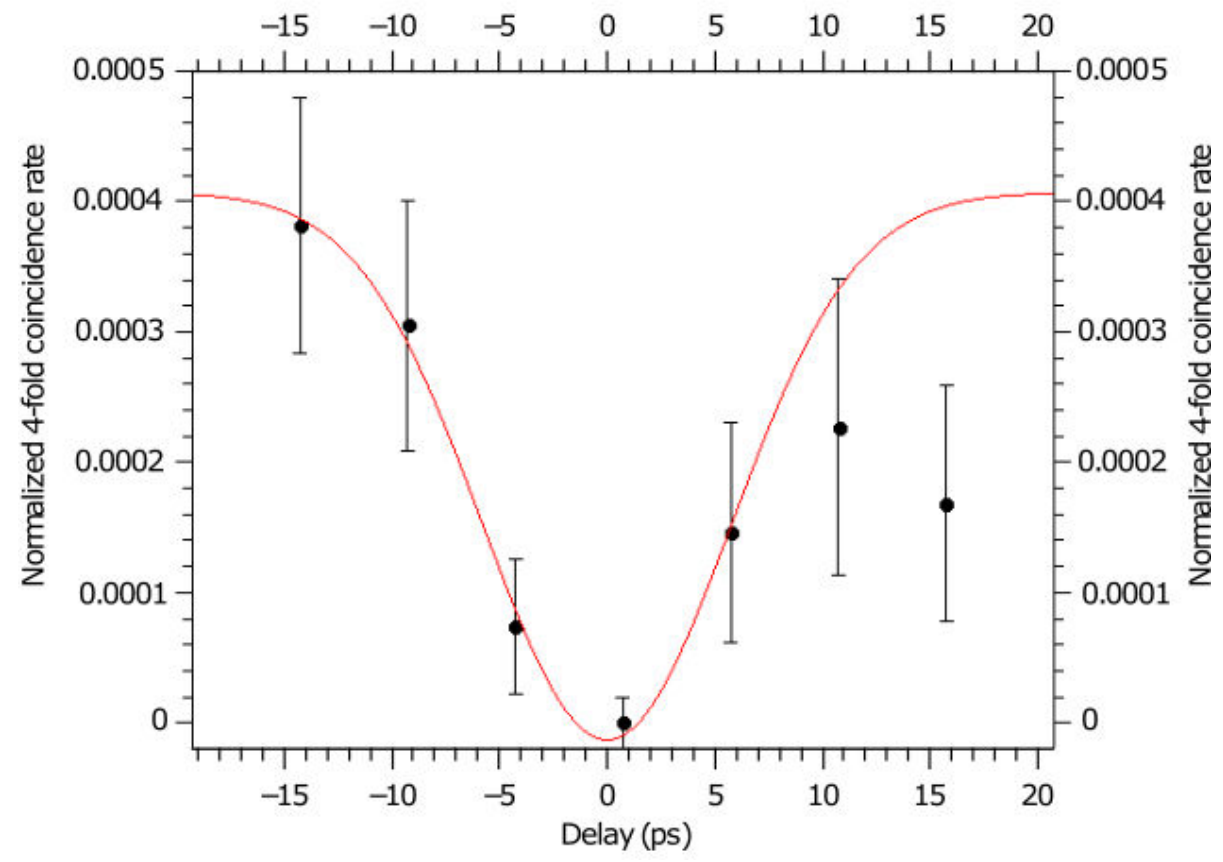

Figure 9.12: The accumulated 4-fold coincidences during 21 hours HOM dip measurement for a total of 7 points. A perfect raw visibility for the HOM dip is observed. The solid line represents a Gaussian fit to the data. 
vious reported works. This can be directly observed through the demonstrated prototype HOM experiment, where opto-electrical conversion stages are not required due to the use of a single master-clock laser. Additionally, operating in the picosecond regime allows us to exploit off-the-shelves, high-performance, and cost-effective telecom components, namely DWDM and FBG filters.

So far, encouraging results have been obtained. A near perfect dip visibility in a HOM experiment is observed thus validating at once our proposed all-optical synchronization method. Such an outcome also highlights the quality of our approach in preparing the single photons required for two-photon interference. As of the moment, extensive efforts have been focused on increasing the overall experimental rate. The use of standard InGaAs APDs impose low pumping regimes and as a consequence, low 4-fold coincidence events. It is our plan in the future to employ a superconducting single photon detector (SSPD), like one that had been used in the realization of the ultra-fast heralded single photon source (UFHSPS, see Chapter 8). In the meantime, the heralding rate is also optimized by multiplexing four free-running IDQ 220 detectors. Once the condition of the experiment is improved, we plan to demonstrate a two-photon interference experiment at long distances whereby our two SPDC stages are separated by $100 \mathrm{~km}$ at high detection rates. Beyond that, it is also our aim to exploit this synchronization configuration for demonstrating long distance entanglement swapping using time-bin qubits. 

Overall summary 



\section{Overall summary}

The 1980's marked the advent of quantum communication. Alain Aspect and his co-workers demonstrated, in a very convincing manner [Aspect et al. 1981, Aspect et al. 1982a, Aspect et al. 1982b], the existence of entanglement that was first introduced in 1935 by the Einstein, Podolsky and Rosen (EPR) trio [Einstein et al. 1935]. Meanwhile in 1984, the field of quantum key distribution (QKD) was officially launched by Charles Bennett and Gilles Brassard through the introduction of the BB84 QKD protocol [Bennett \& Brassard 1984]. Compared to conventional method where the security level of exchanging information is defined by the adversary's computational power, BB84 offers unconditional security by taking advantage of the no-cloning theorem and the concept of complementarity between measurement bases offered exclusively by quantum physics. The conceptuality of QKD was proven through the first experimental, proof-of-concept demonstration performed by Bennett and his co-workers in 1992 [Bennett et al. 1992]. As of December 2015, a team of researchers from the University of Geneva has successfully demonstrated a record QKD distance spanning over $307 \mathrm{~km}$ of optical fibers in a laboratory environment. QKD is also being pushed to be implemented outside laboratories, and it is the aim of this research community to look forward for architectural QKD networks. Across the globe, QKD networks have been established, including, Vienna (SECOQC ${ }^{2}$ ), Tokyo, and Los Alamos. Last but not least, China, as a new big player in this affair, has started installing, presumably the worlds longest QKD network spanning over 2000 kilometers of telecom optical fibers between Beijing and Shanghai. With their enormous resources, China also plans to launch the worlds first satellite-to-ground QKD link in 2016. Besides, integrated QKD systems are also commercially available, notably from companies such as idQuantique (Geneva), SeQureNet (Paris), MagiQ Technologies (New York), and QuintessenceLabs (Australia). Above all, most of the advances presented so far are made possible with the exploitation of photonic qubits at telecom wavelength. Moreover, they can be comfortably manipulated using readily available, high-performance, low-loss, and cost-effective telecom components.

Since the late 1980's, we also have seen the exploitation of bulk and waveguide-based

\footnotetext{
${ }^{2}$ SECOQC stands for Secure Communication based on Quantum Cryptography.
} 
crystals featuring $\chi^{(2)}$ non-linearities, which then emerged as the workhorse toward producing entangled photon pairs. Tremendous efforts have been focused on the study of these kind of materials, making them more reliable, practical and efficient, notably for quantum communication applications at telecom wavelength. Such advances have made entanglement more accessible, and moreover, entanglement can distributed over longer distances [Inagaki et al. 2013]. In addition, high efficiency photon pair sources have induced the development of heralded single photon sources, where high quality single photons at telecom wavelength can be delivered at an arbitrarily high rate.

Within the framework of this thesis, on the basis of exploiting the advantages offered by telecom wavelength, we aimed at contributing to the field of quantum communication by demonstrating two polarization entangled photon pair sources (EPPS), two heralded single photon sources (HSPS), and a genuinely-synchronized quantum relay experiment. In all of our experiments, paired photons at telecom wavelength are generated via SPDC in periodically poled lithium niobate waveguide (PPLN/W).

The first EPPS is based on non-linear type-II interaction in PPLN/W. A full guidedwave approach has been used to compensate the natural birefringence inherent to the PPLN/W and also for deterministic paired photons separation into two telecom channels. Excellent quality of entanglement has been measured through the Bell-CHSH inequality test [Kaiser et al. 2012b]. With the level of simplicity offered by this source, it is, undoubtedly, a good candidate for DWDM-based, Ekert-type QKD implementation [Ekert 1991]. The second EPPS generates paired photons, out of a type-0 non-linear interaction in a PPLN/W, that can be easily engineered in term of their spectral bandwidth and entangled state. As the first EPPS, this particular source is also ready to be implemented in Ekerttype QKD. Even more than that, the photon spectral bandwidth can be tailored down to $25 \mathrm{MHz}$, allowing this source to be implemented in DLCZ protocol along with cold atom quantum memory devices, provided a coherent quantum interface is employed for frequency conversion [Kaiser et al. 2013].

The first HSPS emphasizes, in a miniaturized fashion, spatial multiplexing of four PPLN/Ws in order to reduce multi-photon generation events while maintaining a reasonable pump power. Such unprecedented scability is achieved through hybridization between PPLN/Ws and femtosecond laser direct write technique. Thanks to the spatial multiplex- 
ing, we could demonstrate an almost four times increase in the available heralded single photon rate [Meany et al. 2014]. The second HSPS is capable of delivering heralding rates in the $\mathrm{MHz}$ regime at negligible multi-photon events by performing passive temporal multiplexing on a single PPNL/W. In this case, the pump field for the non-linear process is delivered by a $10 \mathrm{GHz}$ repetition rate telecom laser [Ngah et al. 2015]. Both sources produce single photons at telecom wavelength, thus making them suitable to be employed in long distance QKD links. Besides, the high quality single photons delivered by these sources can be exploited by other branches of quantum information science, namely linear optical quantum computing, and quantum metrology.

Through the quantum relay experiment, we have proposed an efficient way toward synchronizing two remote EPPS. The scheme relies on the use of ultra-fast master clock optical pulses delivered by a $2.5 \mathrm{GHz}$ repetition rate telecom laser, which then serves as pump for entangled paired photons in both sources. These produced optical clock pulses at telecom wavelength is distributed in a fully-guided wave manner thus allowing unprecedented separation between these two sources. Last but not least, working at $2.5 \mathrm{GHz}$ repetition rate regime helps in fighting low success rates experienced by most of quantum protocols due to probabilistic photon pair generation via the process.

As we are much inspired by countless quantum photonic experiments before us, it is our aim that this thesis work, in one way or another, might inspire others for the sole purpose of maintaining the continuous development in the field of quantum communication. 



\section{Single photon detectors and coincidence electronics}

Detecting single photons events is crucial to this thesis work. All of the demonstrated experiments involve the use of indium gallium arsenide (InGaAs) avalanche photo-diodes (APD), and/or superconducting single photon detector (SSPD), as well as coincidence count electronics.

\section{A.1 InGaAs avalanche photo-diodes: working principle}

Generally, an avalanche photo-diode (APD) is based on a P-N junction in a semiconductor which is capable of sustaining reverse bias voltages. In an indium gallium arsenide (InGaAs) APD, for instance, a photon is absorbed in a narrow bandgap n-InGaAs region, and an electron-hole pair is created. The photo-generated hole is then injected into an InP multiplication zone, via a reverse biased voltage, where a charge avalanche is generated. Note that separate absorption and multiplication zones are implemented. Generally in InP, the multiplication, i.e. the avalanche effect is optimized due to low excess noise. Using suitable electronics, the charge avalanche is measured. After signal detection, the avalanche is actively stopped by using electronics. This is done in order to free the detector for the next photon detection. The time required for stopping the avalanche is generally referred as the dead time of the detector in which no photon detection can occur during this period of time. In such a detector, associated dark count rate can be further reduced by cooling down the detector to $200 \mathrm{~K}$, and this can be done through the use of Peltier elements. However, this unfortunately implies longer dead times so as to reduce the probability of having an after-pulse. 


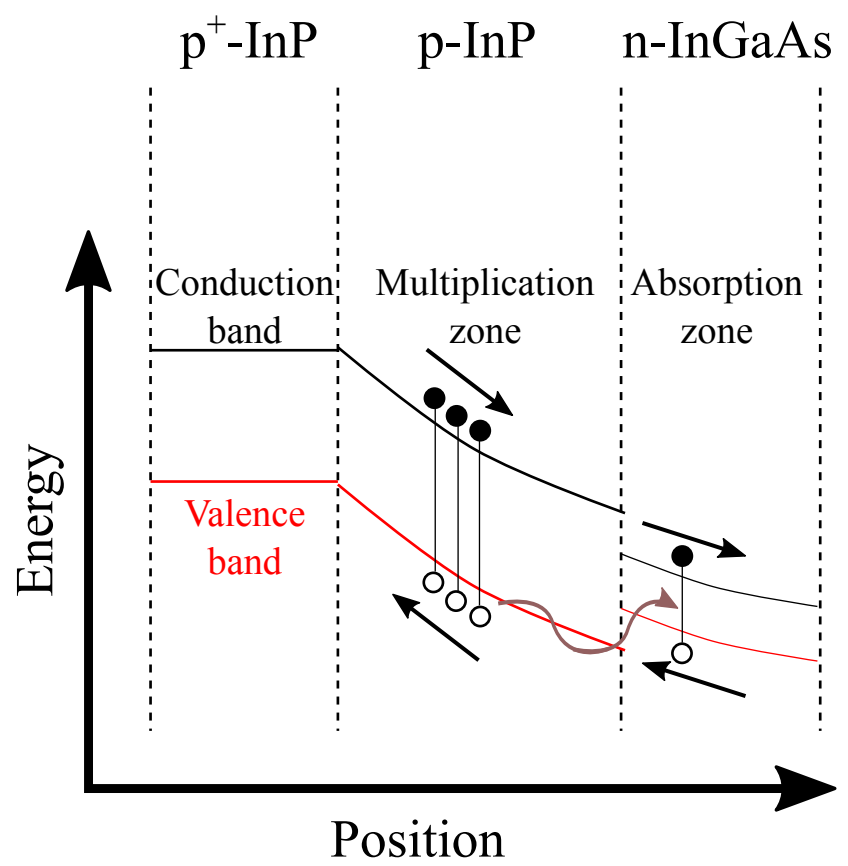

Figure A.1: Separate absorption and multiplication zone. A single photon is absorbed in the n-InGaAs zone and electron-hole pair is created. The hole is then injected into p-InP region. Here, a charge avalanche is generated, followed by a detection in the $\mathrm{p}^{+}-\mathrm{InP}$ zone. Due to InGaAs exhibit narrow bandgap compared to others,

\section{A.2 Superconducting single photon detectors: working prin- ciple}

Superconducting single photon detector (SSPD) generally consists of ultra-thin niobium nitride film (with a typical wide of $100 \mathrm{~nm}$, covering an area of several $\mu \mathrm{m}^{2}$ ), cooled down in helium liquid until a cryogenic temperature range between $1.5 \mathrm{~K}$ to $4 \mathrm{~K}$. In this temperature range, the film is superconducting and it has the capacity of conducting electricity with zero resistance. When the film becomes resistive, it is operated just below its critical current which refers, for instance, to the case of single photon absorption by the thin film. In this case, a hot spot is locally created thus perturbing the current distribution of the thin film. Consequently, the film loses its superconducting capacity for a short amount of time, and a short voltage pulse is observed. This pulse can be amplified by suitable electronic amplifier thus announcing the detection of a single photon.

For our ultra-fast heralded single photon source (UFHSPS) experiment in Chapter 8, an SSPD from Scoentel (model LTD24/30-008) is used detect heralding photons at $1537.4 \mathrm{~nm}$. 
(a)

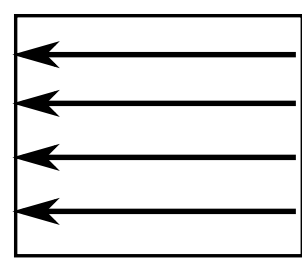

(c)

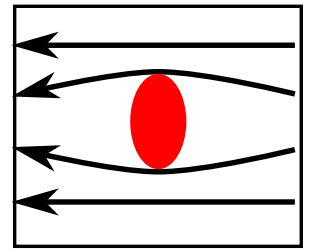

(b)

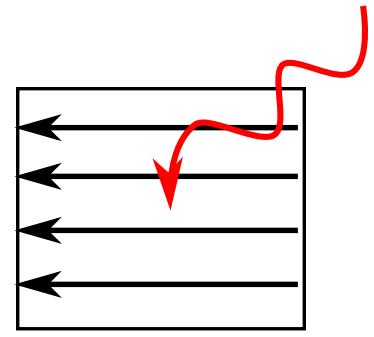

(d)

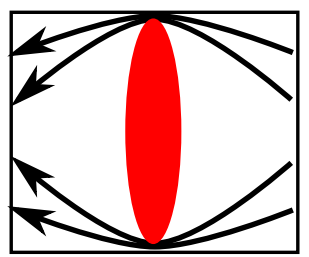

Figure A.2: Working principle of a superconducting single photon detector. (a): The material is cooled down to cryogenic temperature of $2 \mathrm{~K}$ and the structure is now in superconducting state. The straight lines indicate that the current is unperturbed. (b): A photon is absorbed by the thin film. (c): A hot spot is created by the absorbed photon and the current distribution is disturbed. (d): At one point, the critical current density is reached. The material then loses its superconducting capacity and a short voltage pulse is observed.

The efficiency of this detector as a function of the registered dark count rate is shown in Fig.A.3. 


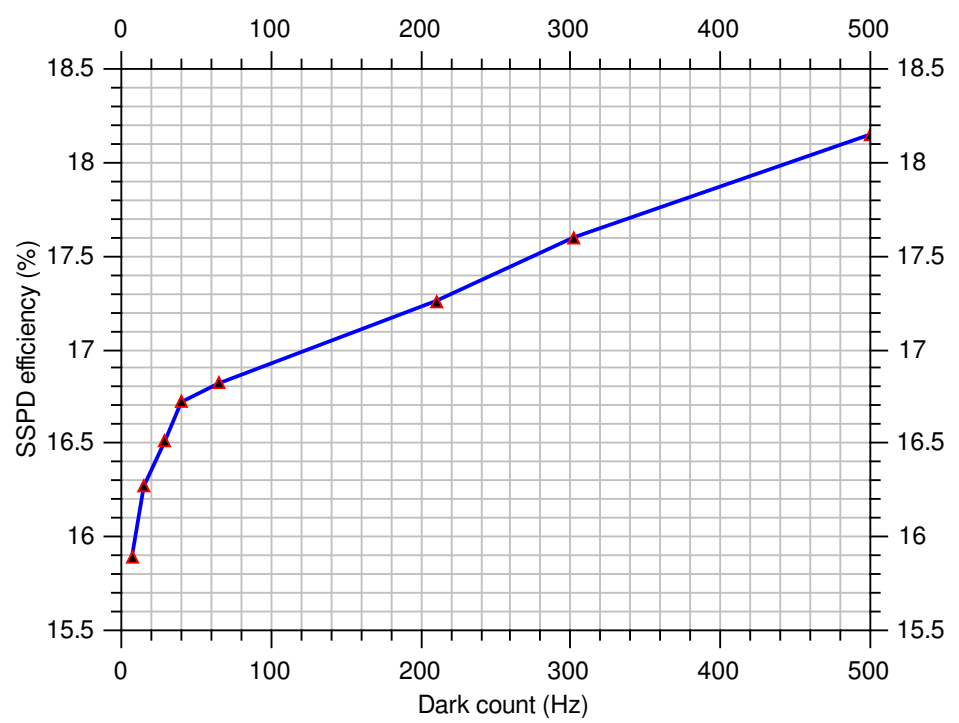

Figure A.3: Measured SSPD efficiency as a function of the registered dark count rate. The characterization was done using attenuated laser beam at $1537.4 \mathrm{~nm}$.

\section{A.3 Figures-of-merit and comparison}

Several main features, are associated with a single photon detectors:

- Quantum detection efficiency $\eta_{d}$ determining the percentage of the detected photons;

- Dark count probability $p_{d c}$;

- Detector timing jitter $\tau_{j}$ representing the detection time uncertainty;

- Dead time $D T$ in which no photon can be detected during this specific interval;

- Operating mode $M$;

- Maximum count rate $R_{\max }$;

- Wavelength range $\lambda$.

Concurrently, we use the above parameters to provide comparisons between all the employed single photon detectors for this thesis work (see Tab.A.1). 
Table A.1: Figures-of-merit for employed single photon detectors.

\begin{tabular}{lllll}
\hline & IDQ 201 & IDQ 210 & IDQ 220 & Scontel SSPD \\
\hline$\eta_{d}$ at $1550 \mathrm{~nm}$ & $10-25 \%$ & $5-25 \%$ (gated) & $20 \%$ & $17 \%$ \\
$p_{d c} ;$ & $10^{-5} \mathrm{~ns}$ & $10^{-6} \mathrm{~ns}$ & $10^{-6} \mathrm{~ns}$ & $10^{-8} \mathrm{~ns}$ \\
$\tau_{j}$ at max. ${ }^{1} \eta_{d}$ & $150 \mathrm{ps}$ & $210 \mathrm{ps}$ & $160 \mathrm{ps}$ & $57 \mathrm{ps}$ \\
Typical $D T$ & $40 \mu \mathrm{s}$ & $4-10 \mu \mathrm{s}$ & $7-10 \mu \mathrm{s}$ & $<2 \mathrm{~ns}$ \\
$M$ & gated & gated/free-running & free-running & free-running \\
$R_{\text {max }}$ & & & $100 \mathrm{kHz}$ & $9-10 \mathrm{MHz}$ \\
$\lambda$ & $1300-1600 \mathrm{~nm}$ & $1300-1600 \mathrm{~nm}$ & $1300-1600 \mathrm{~nm}$ & visible - near-infrared \\
\hline
\end{tabular}

\section{A.4 Coincidence detection scheme}

The importance of detecting a coincidence has been portrayed throughout this manuscript. For this thesis work, two type of devices have been employed, a time-to-amplitude converter (TAC, ORTEC 567) and a logical AND gate (\&, ORTEC CO4020).

With the TAC, a delay $\Delta T$ between "start" and "stop" signals can be transformed into amplitude. With an acquisition card attached to a computer, a coincidence histogram can be constructed. In the case of, for instance, time-correlated photon pairs, a coincidence peak is observed. However, the TAC device can only analyze positive delays such that the "stop" signal should be adequately delayed with respect to the "start" signal. As a side note, between two input signals, the "start" input should be assigned with low-count one, so as to prevent the saturation of the TAC.

On the other hand, the logical AND gate is useful when working with signals in the MHZ regime. However, unlike TAC, the logical gate do not provide the $\Delta T$. It only deliver conventional electrical pulse when a coincidence is detected. As there is no additional information provided by such device, coincidence peak cannot be observed. 



\section{Bibliography}

[Aboussouan et al. 2010] Pierre Aboussouan, Olivier Alibart, Daniel B. Ostrowsky, Pascal Baldi and Sébastien Tanzilli. High-visibility two-photon interference at a telecom wavelength using picosecond-regime separated sources. Physical Review A - Atomic, Molecular, and Optical Physics, vol. 81, no. 2, 2010. (Cited on pages 3, 58, 130, 179, $181,182,184,191$, and 195.)

[Acín et al. 2007] Antonio Acín, Nicolas Brunner, Nicolas Gisin, Serge Massar, Stefano Pironio and Valerio Scarani. Device-independent security of quantum cryptography against collective attacks. Physical Review Letters, vol. 98, no. 23, 2007. (Cited on page 43.)

[Aktas et al. 2015] Djeylan Aktas, Sébastien Tanzilli, Anthony Martin, Gilles Pütz, Rob Thew and Nicolas Gisin. Demonstration of Quantum Nonlocality in the Presence of Measurement Dependence. Physical Review Letters, vol. 114, no. 22, page 220404, jun 2015. (Cited on pages 34 and 43.)

[Albrecht et al. 2013] Roland Albrecht, Alexander Bommer, Christian Deutsch, Jakob Reichel and Christoph Becher. Coupling of a Single Nitrogen-Vacancy Center in Diamond to a Fiber-Based Microcavity. Physical Review Letters, vol. 110, no. 24, page 243602, June 2013. (Cited on page 128.)

[Alibart et al. 2005] O Alibart, D B Ostrowsky, P Baldi and S Tanzilli. High-performance guided-wave asynchronous heralded single-photon source. Optics letters, vol. 30, no. 12, pages 1539-1541, 2005. (Cited on pages 2, 129, 130, and 167.)

[Allevi et al. 2010] A. Allevi, A. Andreoni, F. A. Beduini, M. Bondani, M. G. Genoni, S. Olivares and M. G. A. Paris. Conditional measurements on multimode pairwise entangled states from spontaneous parametric downconversion. EPL (Europhysics Letters), vol. 92, no. 2, page 20007, October 2010. (Cited on page 169.)

[Almeida et al. 2011] A. J. Almeida, S. R. Carneiro, N. A. Silva, N. J. Muga and A. N. Pinto. Polarization-entangled photon pairs using spontaneous four-wave mixing in a 
fiber loop. In 2011 IEEE EUROCON - International Conference on Computer as a Tool, pages 1-4, 2011. (Cited on page 65.)

[Armstrong et al. 1962] J A Armstrong, N Bloembergen, J Ducuing and P S Pershan. Interactions between Light Waves in a Nonlinear Dielectric. Phys. Rev., vol. 127, no. 6, page 1918, 1962. (Cited on page 68.)

[Aspect et al. 1981] Alain Aspect, Philippe Grangier and Gérard Roger. Experimental tests of realistic local theories via Bell's theorem. Physical Review Letters, vol. 47, no. 7, pages 460-463, 1981. (Cited on pages 2, 34, 61, and 205.)

[Aspect et al. 1982a] A Aspect, P Grangier and G Roger. Experimental Realization of \{Einstein-Podolsky-Rosen-Bohm\} \{G\}edankenexperiment: A New Violation of $\{B\}$ ell's Inequalities. Phys. Rev. Lett., vol. 49, no. 2, pages 91-94, 1982. (Cited on pages $2,34,61$, and 205.)

[Aspect et al. 1982b] Alain Aspect, Jean Dalibard and Gerard Roger. Experimental test of Bell's inequalities using time-varying analyzers. Physical Review Letters, vol. 49, no. 25, pages 1804-1807, 1982. (Cited on pages 2, 61, and 205.)

[Autebert et al. 2015] C. Autebert, G. Boucher, F. Boitier, A. Eckstein, I. Favero, G. Leo and S. Ducci. Photon pair sources in AlGaAs: from electrical injection to quantum state engineering. Journal of Modern Optics, pages 1-7, 2015. (Cited on page 65.)

[Avella et al. 2011] A. Avella, G. Brida, I. P. Degiovanni, M. Genovese, M. Gramegna, L. Lolli, E. Monticone, C. Portesi, M. Rajteri, M. L. Rastello, E. Taralli, P. Traina and M. White. Self consistent, absolute calibration technique for photon number resolving detectors, 2011. (Cited on pages 2 and 126.)

[Bao et al. 2008] X.-H. Bao, Y Qian, J Yang, H Zhang, Z.-B. Chen, T Yang and J.-W. Pan. Generation of \{Narrow-Band\} \{Polarization-Entangled\} Photon Pairs for Atomic Quantum Memories. Phys. Rev. Lett., vol. 101, no. 19, page 190501, 2008. (Cited on page 121.) 
[Bao et al. 2012] X.-H. Bao, A Reingruber, P Dietrich, J Rui, A Dück, T Strassel, L Li, N.-L. Liu, B Zhao and J.-W. Pan. Efficient and long-lived quantum memory with cold atoms inside a ring cavity. Nature Phys., 2012. (Cited on page 104.)

[Bell 1964] J S Bell. On the \{Einstein-Podolsky-Rosen\} Paradox. Physics \{(Long\} Island City, \{N.Y.)\}, vol. 1, pages 195-200, 1964. (Cited on pages 1, 34, and 61.)

[Bengtsson 2007] Ingemar Bengtsson. Three Ways to Look at Mutually Unbiased Bases. In AIP Conference Proceedings, volume 889, pages 40-51. AIP, February 2007. (Cited on page 22.)

[Bennett \& Brassard 1984] C H Bennett and G Brassard. Quantum Cryptography: Public Key Distribution and Coin Tossing. Proceedings of the $\{$ IEEE $\}$ International Conference on Computers, Systems and Signal Processing, Bangalore, India, page 175, 1984. (Cited on pages 2, 40, and 205.)

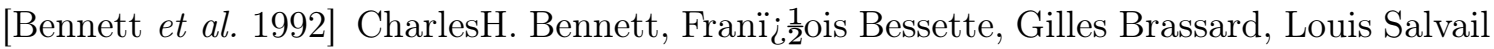
and John Smolin. Experimental quantum cryptography. Journal of Cryptology, vol. 5, no. 1, pages 3-28, 1992. (Cited on page 205.)

[Bennett et al. 1993] C H Bennett, G Brassard, C Crépeau, R Jozsa, A Peres and W K Wootters. Teleporting an unknown quantum state via dual classical and \{EinsteinPodolsky-Rosen\} channels. Phys. Rev. Lett., vol. 70, no. 13, pages 1895-1899, 1993. (Cited on page 54.)

[Bennett et al. 2005] A Bennett, D Unitt, P Atkinson, D Ritchie and A Shields. High performance single photon sources from photolithographically defined pillar microcavities. Optics express, vol. 13, no. 1, pages 50-55, 2005. (Cited on page 127.)

[Bennett et al. 2008] A. J. Bennett, R. B. Patel, A. J. Shields, K. Cooper, P. Atkinson, C. A. Nicoll and D. A. Ritchie. Indistinguishable photons from a diode. Applied Physics Letters, vol. 92, no. 19, 2008. (Cited on page 127.)

[Bimbard et al. 2014] Erwan Bimbard, Rajiv Boddeda, Nicolas Vitrant, Andrey Grankin, Valentina Parigi, Jovica Stanojevic, Alexei Ourjoumtsev and Philippe Grangier. Homodyne tomography of a single photon retrieved on demand from a cavity-enhanced 
cold atom memory. Physical Review Letters, vol. 112, no. 3, pages 1-5, 2014. (Cited on page 104.)

[bin Abdul Khir 2012] Mohd Fared bin Abdul Khir. Implementation of two-way free space quantum key distribution. Optical Engineering, vol. 51, no. 4, page 045006, apr 2012. (Cited on page 36.)

[Black et al. 2005] A T Black, J K Thompson and V Vuletic. On-Demand Superradiant Conversion of Atomic Spin Gratings into Single Photons with High Efficiency. Phys. Rev. Lett., vol. 95, no. 13, page 133601, 2005. (Cited on page 104.)

[Bogaerts et al. 2006] Wim Bogaerts, Pieter Dumon, Dries Van Thourhout, Dirk Taillaert, Patrick Jaenen, Johan Wouters, Stephan Beckx, Vincent Wiaux and Roel G. Baets. Compact wavelength-selective functions in silicon-on-insulator photonic wires. IEEE Journal on Selected Topics in Quantum Electronics, vol. 12, no. 6, pages 1394-1401, 2006. (Cited on page 130.)

[Boitier et al. 2014] Fabien Boitier, Adeline Orieux, Claire Autebert, Aristide Lemaître, Elisabeth Galopin, Christophe Manquest, Carlo Sirtori, Ivan Favero, Giuseppe Leo and Sara Ducci. Electrically Injected Photon-Pair Source at Room Temperature. Physical Review Letters, vol. 112, no. 18, page 183901, 2014. (Cited on page 65.)

[Boschi et al. 1998] D Boschi, S Branca, F De Martini, L Hardy and S Popescu. Experimental Realization of Teleporting an Unknown Pure Quantum State via Dual Classical and Einstein-Podolsky-Rosen Channels. Phys. Rev. Lett., vol. 80, no. 6, pages 11211125, February 1998. (Cited on pages 56 and 62.)

[Bouwmeester et al. 1997] Dik Bouwmeester, Jian-Wei Pan, Klaus Mattle, Manfred Eibl, Harald Weinfurter and Anton Zeilinger. Experimental quantum teleportation. Nature, vol. 390, no. 6660, pages 575-579, 1997. (Cited on pages 56 and 62.)

[Brassard et al. 2000] Gilles Brassard, Norbert Lütkenhaus, Tal Mor and Barry C. Sanders. Limitations on practical quantum cryptography. Physical Review Letters, vol. 85, no. 6, pages 1330-1333, 2000. (Cited on page 126.) 
[Brendel et al. 1992] J Brendel, E Mohler and W Martienssen. Experimental Test of Bell's Inequality for Energy and Time. Europhys. Lett., vol. 20, page 575, 1992. (Cited on page 109.)

[Brendel et al. 1999] J. Brendel, N. Gisin, W. Tittel and H. Zbinden. Pulsed Energy-Time Entangled Twin-Photon Source for Quantum Communication. Physical Review Letters, vol. 82, no. 12, pages 2594-2597, March 1999. (Cited on page 38.)

[Brida et al. 2012] G. Brida, I. P. Degiovanni, M. Genovese, F. Piacentini, P. Traina, A. Della Frera, A. Tosi, A. Bahgat Shehata, C. Scarcella, A. Gulinatti, M. Ghioni, S. V. Polyakov, A. Migdall and A. Giudice. An extremely low-noise heralded singlephoton source: A breakthrough for quantum technologies. Applied Physics Letters, vol. 101, no. 22, page 221112, November 2012. (Cited on pages 129 and 170.)

[Broome et al. 2011] M. A. Broome, M. P. Almeida, A. Fedrizzi and A. G. White. Reducing multi-photon rates in pulsed down-conversion by temporal multiplexing, 2011. (Cited on page 157.)

[Broome et al. 2013] Matthew A. Broome, Alessandro Fedrizzi, Saleh Rahimi-Keshari, Justin Dove, Scott Aaronson, Timothy C. Ralph and Andrew G. White. Photonic boson sampling in a tunable circuit. Science, vol. 339, page 794, 2013. (Cited on pages 2 and 126.)

[Brouri et al. 2000] R Brouri, A Beveratos, J P Poizat and P Grangier. Photon antibunching in the fluorescence of individual color centers in diamond. Optics letters, vol. 25, no. 17, pages 1294-1296, 2000. (Cited on page 127.)

[Buse et al. 2007] K. Buse, J. Imbrock, E. Krätzig and K. Peithmann. Photorefractive effects in $\mathrm{LiNbO}_{3}$ and $\mathrm{LiTaO}_{3}$. In P. Günther and J. P. Huignard, editeurs, Photorefractive Materials and Their Applications 2. Springer-Verlag New York, New York, 2007. (Cited on page 191.)

[Bussières et al. 2014] Félix Bussières, Christoph Clausen, Alexey Tiranov, Boris Korzh, Varun B. Verma, Sae Woo Nam, Francesco Marsili, Alban Ferrier, Philippe Goldner, Harald Herrmann, Christine Silberhorn, Wolfgang Sohler, Mikael Afzelius and 
Nicolas Gisin. Quantum teleportation from a telecom-wavelength photon to a solidstate quantum memory. Nature Photonics, vol. 8, no. 10, pages 775-778, September 2014. (Cited on page 56.)

[Castaldini et al. 2009] Davide Castaldini, Paolo Bassi, Pierre Aschieri, Sorin Tascu, Marc De Micheli and Pascal a Baldi. High performance mode adapters based on segmented SPE:LiNbO3 waveguides. Optics express, vol. 17, no. 20, pages 17868-73, 2009. (Cited on page 97.)

[Chanelière et al. 2005a] T Chanelière, D N Matsukevich, S D Jenkins, S-Y Lan, T a B Kennedy and a Kuzmich. Storage and retrieval of single photons transmitted between remote quantum memories. Nature, vol. 438, no. 7069, pages 833-836, 2005. (Cited on page 66.$)$

[Chanelière et al. 2005b] T Chanelière, D N Matsukevich, S D Jenkins, S.-Y. Lan, T A B Kennedy and A Kuzmich. Storage and retrieval of single photons transmitted between remote quantum memories. Nature, vol. 438, no. 7069, pages 833-836, 2005. (Cited on page 104.)

[Chanvillard et al. 2000] L. Chanvillard, P. Aschièri, P. Baldi, D. B. Ostrowsky, M. de Micheli, L. Huang and D. J. Bamford. Soft proton exchange on periodically poled LiNbO[sub 3]: A simple waveguide fabrication process for highly efficient nonlinear interactions. Applied Physics Letters, vol. 76, no. 9, page 1089, 2000. (Cited on page 70.)

[Chen et al. 2007] J Chen, G Wu, Y Li, E Wu and H Zeng. Active polarization stabilization in optical fibers suitable for quantum keydistribution. Opt. Express, vol. 15, no. 26, pages 17928-17936, 2007. (Cited on pages 36, 66, and 100.)

[Chen et al. 2008] Y.-A. Chen, S Chen, Z.-S. Yuan, B Zhao, C.-S. Chuu, J Schmiedmayer and J.-W. Pan. Memory-built-in quantum teleportation with photonic and atomic qubits. Nature Phys., vol. 4, no. 2, pages 103-107, 2008. (Cited on page 104.) 
[Chen et al. 2009] J Chen, G Wu, L Xu, X Gu, E Wu and H Zeng. Stable quantum key distribution with active polarization control based on time-division multiplexing. New Journal of Physics, vol. 11, no. 6, page 065004, 2009. (Cited on pages 36, 66, and 100.)

[Choi et al. 2008] K S Choi, H Deng, J Laurat and H J Kimble. Mapping photonic entanglement into and out of a quantum memory. Nature, vol. 452, no. 7183, pages 67-71, 2008. (Cited on page 104.)

[Choi et al. 2010] K S Choi, A Goban, S B Papp, S J van Enk and H J Kimble. Entanglement of spin waves among four quantum memories. Nature, vol. 468, no. 7322, pages 412-416, 2010. (Cited on page 104.)

[Clark et al. 2012] Alex S. Clark, Matthew J. Collins, Alexander C. Judge, Eric C. Mägi, Chunle Xiong and Benjamin J. Eggleton. Raman scattering effects on correlated photon-pair generation in chalcogenide. Optics Express, vol. 20, no. 15, page 16807, July 2012. (Cited on page 130.)

[Clark et al. 2013] Alex S. Clark, Chad Husko, Matthew J. Collins, Gaelle Lehoucq, Stéphane Xavier, Alfredo De Rossi, Sylvain Combrié, Chunle Xiong and Benjamin J. Eggleton. Heralded single-photon source in a III-V photonic crystal. Optics Letters, vol. 38, no. 5, pages 649-651, 2013. (Cited on page 130.)

[Clarke et al. 2011] Patrick J Clarke, Robert J Collins, Philip A Hiskett, María-José GarcíaMartínez, Nils J Krichel, Aongus McCarthy, Michael G Tanner, John A O'Connor, Chandra M Natarajan, Shigehito Miki, Masahide Sasaki, Zhen Wang, Mikio Fujiwara, Ivan Rech, Massimo Ghioni, Angelo Gulinatti, Robert H Hadfield, Paul D Townsend and Gerald S Buller. Analysis of detector performance in a gigahertz clock rate quantum key distribution system. New Journal of Physics, vol. 13, no. 7, page 075008, July 2011. (Cited on page 164.)

[Clausen et al. 2011] Christoph Clausen, Imam Usmani, Félix Bussières, Nicolas Sangouard, Mikael Afzelius, Hugues de Riedmatten and Nicolas Gisin. Quantum storage of photonic entanglement in a crystal. Nature, vol. 469, no. 7331, pages 508-11, 2011. (Cited on page 104.) 
[Clauser \& Shimony 1978] J F Clauser and A Shimony. Bell's theorem. Experimental tests and implications. Reports on Progress in Physics, vol. 41, no. 12, pages 1881-1927, dec 1978. (Cited on page 61.)

[Clauser et al. 1969] John F. Clauser, Michael A. Horne, Abner Shimony and Richard A. Holt. Proposed Experiment to Test Local Hidden-Variable Theories. Physical Review Letters, vol. 23, no. 15, pages 880-884, 1969. (Cited on pages 2, 31, and 87.)

[Clemmen et al. 2009] S Clemmen, K Phan Huy, W Bogaerts, R G Baets, Ph Emplit and $\mathrm{S}$ Massar. Continuous wave photon pair generation in silicon-on-insulator waveguides and ring resonators. Optics express, vol. 17, no. 19, pages 16558-70, September 2009. (Cited on pages 65 and 130.)

[Collins et al. 2005] D Collins, N Gisin and H de Riedmatten. Quantum relays for long distance quantum cryptography. J. Mod. Opt., vol. 52, pages 735-753, 2005. (Cited on pages 3 and 175.)

[Collins et al. 2013] M J Collins, C Xiong, I H Rey, T D Vo, J He, S Shahnia, C Reardon, T F Krauss, M J Steel, a S Clark and B J Eggleton. Integrated spatial multiplexing of heralded single-photon sources. Nature communications, vol. 4, no. May, page 2582, 2013. (Cited on pages 130, 152, and 156.)

[Crespi et al. 2013] Andrea Crespi, Giacomo Corrielli, Giuseppe Della Valle, Roberto Osellame and Stefano Longhi. Dynamic band collapse in photonic graphene. New Journal of Physics, vol. 15, 2013. (Cited on page 140.)

[Da Cunha Pereira et al. 2013] Marcelo Da Cunha Pereira, Francisco E Becerra, Boris L Glebov, Jingyun Fan, Sae Woo Nam and Alan Migdall. Demonstrating highly symmetric single-mode, single-photon heralding efficiency in spontaneous parametric downconversion. Optics letters, vol. 38, no. 10, pages 1609-11, May 2013. (Cited on page 156.)

[Davanço et al. 2012] Marcelo Davanço, Jun Rong Ong, Andrea Bahgat Shehata, Alberto Tosi, Imad Agha, Solomon Assefa, Fengnian Xia, William M J Green, Shayan 
Mookherjea and Kartik Srinivasan. Telecommunications-band heralded single photons from a silicon nanophotonic chip. Applied Physics Letters, vol. 100, no. 26, 2012. (Cited on page 130.)

[Davis et al. 1996] K. M. Davis, K. Miura, N. Sugimoto and K. Hirao. Writing waveguides in glass with a femtosecond laser. Optics Letters, vol. 21, no. 21, page 1729, November 1996. (Cited on page 140.)

[de Riedmatten et al. 2002] H de Riedmatten, I Marcikic, H Zbinden and N Gisin. Creating high dimensional time-bin entanglement using mode-locked lasers. Quant. Inf. Comp., vol. 2, pages 425-433, 2002. (Cited on page 38.)

[de Riedmatten et al. 2003] H de Riedmatten, I Marcikic, W Tittel, H Zbinden and N Gisin. Quantum interference with photon pairs created in spatially separated sources. Phys. Rev. A, vol. 67, no. 2, page 22301, 2003. (Cited on page 180.)

[de Riedmatten et al. 2004a] H de Riedmatten, I Marcikic, W Tittel, H Zbinden, D Collins and N Gisin. Long Distance Quantum Teleportation in a Quantum Relay Configuration. Phys. Rev. Lett., vol. 92, no. 4, page 47904, 2004. (Cited on page 56.)

[de Riedmatten et al. 2004b] H de Riedmatten, V Scarani, I Marcikic, A Ac〉'in, W Tittel, H Zbinden and N Gisin. Two independent photon pairs versus four-photon entangled states in parametric down conversion. J. Mod. Opt., vol. 51, pages 1637-1649, 2004. (Cited on pages 127, 156, and 169.)

[De Riedmatten et al. 2005] H. De Riedmatten, I. Marcikic, J. A W Van Houwelingen, W. Tittel, H. Zbinden and N. Gisin. Long-distance entanglement swapping with photons from separated sources. Physical Review A - Atomic, Molecular, and Optical Physics, vol. 71, no. 5, 2005. (Cited on pages 57, 179, and 180.)

[Duan et al. 2001] L M Duan, M D Lukin, J I Cirac and P Zoller. Long-distance quantum communication with atomic ensembles and linear optics. Nature, vol. 414, no. 6862, pages 413-418, 2001. (Cited on pages 3 and 66.) 
[Dyer et al. 2009] Shellee D Dyer, Burm Baek and Sae Woo Nam. High-brightness, lownoise, all-fiber photon pair source. Optics express, vol. 17, no. 12, pages 10290-10297, 2009. (Cited on pages 65 and 129.)

[Dynes et al. 2009] J F Dynes, H Takesue, Z L Yuan, a W Sharpe, K Harada, T Honjo, H Kamada, O Tadanaga, Y Nishida, M Asobe and a J Shields. Efficient entanglement distribution over 200 kilometers. Optics express, vol. 17, no. 14, pages 11440-11449, 2009. (Cited on page 66.)

[Eaton et al. 2009] Shane M. Eaton, Wei Jen Chen, Haibin Zhang, Rajiv Iyer, Jianzhao Li, Mi Li Ng, Stephen Ho, J. Stewart Aitchison and Peter R. Herman. Spectral loss characterization of femtosecond laser written waveguides in glass with application to demultiplexing of 1300 and 1550 nm wavelengths. Journal of Lightwave Technology, vol. 27, no. 9, pages 1079-1085, 2009. (Cited on page 143.)

[Einstein et al. 1935] A Einstein, B Podolsky and N Rosen. Can \{Quantum-Mechanical\} Description of Physical Reality Be Considered Complete? Phys. Rev., vol. 47, pages 777-780, 1935. (Cited on pages 1, 28, 61, and 205.)

[Ekert 1991] A K Ekert. Quantum cryptography based on $\{$ B\}ell's theorem. Phys. Rev. Lett., vol. 67, no. 6, pages 661-663, 1991. (Cited on pages 2, 42, and 206.)

[Erven et al. 2008] C Erven, C Couteau, R Laflamme and G Weihs. Entangled quantum key distribution over two free-space optical links. Optics express, vol. 16, no. 21, pages 16840-16853, 2008. (Cited on page 62.)

[Fan et al. 2005] J Fan, A Migdall and L J Wang. Efficient generation of correlated photon pairs in a microstructure fiber. Optics letters, vol. 30, no. 24, pages 3368-3370, 2005. (Cited on pages 65 and 129.)

[Faraon et al. 2011] Andrei Faraon, Paul E. Barclay, Charles Santori, Kai-Mei C. Fu and Raymond G. Beausoleil. Resonant enhancement of the zero-phonon emission from a colour centre in a diamond cavity. Nature Photonics, vol. 5, no. 5, pages 301-305, April 2011. (Cited on page 128.) 
[Fasel et al. 2002] Sylvain Fasel, Nicolas Gisin, Grégoire Ribordy, Valerio Scarani and Hugo Zbinden. Quantum cloning with an optical fiber amplifier. Physical review letters, vol. 89, no. 10, page 107901, 2002. (Cited on page 25.)

[Fasel et al. 2004] S Fasel, O Alibart, S Tanzilli, P Baldi, A Beveratos, N Gisin and H Zbinden. High-quality asynchronous heralded single-photon source at telecom wavelength. New Journal of Physics, vol. 6, page 163, November 2004. (Cited on pages 2 and 129.)

[Fattal et al. 2004a] D Fattal, E Diamanti, K Inoue and Y Yamamoto. Quantum teleportation with a quantum dot single photon source. Physical review letters, vol. 92, no. 3, page 037904, 2004. (Cited on page 128.)

[Fattal et al. 2004b] David Fattal, Kyo Inoue, Jelena Vucković, Charles Santori, Glenn S Solomon and Yoshihisa Yamamoto. Entanglement formation and violation of Bell's inequality with a semiconductor single photon source. Physical review letters, vol. 92, no. 3, page 037903,2004 . (Cited on page 128.)

[Fejer et al. 1992] M.M. Fejer, G.a. Magel, D.H. Jundt and R.L. Byer. Quasi-phase-matched second harmonic generation: tuning and tolerances. IEEE Journal of Quantum Electronics, vol. 28, no. 11, pages 2631-2654, 1992. (Cited on page 69.)

[Fiorentino et al. 2002] Marco Fiorentino, Paul L. Voss, Jay E. Sharping and Prem Kumar. All-fiber photon-pair source for quantum communications. IEEE Photonics Technology Letters, vol. 14, no. 7, pages 983-985, 2002. (Cited on pages 65 and 129.)

[Fouchet et al. 1987] S. Fouchet, a. Carenco, C. Daguet, R. Guglielmi and L. Riviere. Wavelength dispersion of Ti induced refractive index change in $\operatorname{LiNbO}<$ inf $>3</$ inf $>$ as a function of diffusion parameters. Journal of Lightwave Technology, vol. 5, no. 5, 1987. (Cited on page 70.)

[Franken \& Ward 1963] P. A. Franken and J. F. Ward. Optical harmonics and nonlinear phenomena. Reviews of Modern Physics, vol. 35, no. 1, pages 23-39, 1963. (Cited on page 68 .) 
[Franson 1989] J D Franson. Bell inequality for position and time. Phys. Rev. Lett., vol. 62, no. 19, pages 2205-2208, 1989. (Cited on pages 66 and 109.)

[Freedman \& Clauser 1972] S J Freedman and J F Clauser. Experimental Test of Local \{Hidden-Variable\} Theories. Phys. Rev. Lett., vol. 28, no. 14, page 938, 1972. (Cited on pages 2, 33, and 61.)

[Fujii et al. 2007] Go Fujii, Naoto Namekata, Masayuki Motoya, Sunao Kurimura and Shuichiro Inoue. Bright narrowband source of photon pairs at optical telecommunication wavelengths using a type-II periodically poled lithium niobate waveguide. Optics express, vol. 15, no. 20, pages 12769-12776, 2007. (Cited on page 64.)

[Fulconis et al. 2005] J. Fulconis, O. Alibart, W. Wadsworth, P. Russell and J. Rarity. High brightness single mode source of correlated photon pairs using a photonic crystal fiber. Opt. Express, vol. 13, no. 19, pages 7572-7582, 2005. (Cited on pages 65 and 129.)

[Gabriel et al. 2010] Christian Gabriel, Christoffer Wittmann, Denis Sych, Ruifang Dong, Wolfgang Mauerer, Ulrik L. Andersen, Christoph Marquardt and Gerd Leuchs. A generator for unique quantum random numbers based on vacuum states. Nature Photonics, vol. 4, no. 10, pages 711-715, 2010. (Cited on page 45.)

[Ghosh \& Mandel 1987] R. Ghosh and L. Mandel. Observation of nonclassical effects in the interference of two photons, 1987. (Cited on page 68.)

[Giordmaine 1962] J. A. Giordmaine. Mixing of Light Beams in Crystals. Physical Review Letters, vol. 8, no. 1, pages 19-20, jan 1962. (Cited on page 68.)

[Gisin et al. 2002] N Gisin, G Ribordy, W Tittel and H Zbinden. Quantum cryptography. Rev. Mod. Phys., vol. 74, no. 1, pages 145-195, 2002. (Cited on pages 2, 3, 42, 51, and 175.)

[Gisin et al. 2004] Nicolas Gisin, Gregoire Ribordy, Hugo Zbinden, Damien Stucki, Nicolas Brunner and Valerio Scarani. Towards practical and fast Quantum Cryptography. nov 2004. (Cited on page 2.) 
[Gisin et al. 2010] Nicolas Gisin, Stefano Pironio and Nicolas Sangouard. Proposal for implementing device-independent quantum key distribution based on a heralded qubit amplifier. Physical Review Letters, vol. 105, no. 7, 2010. (Cited on page 43.)

[Goebel et al. 2008] Alexander M. Goebel, Claudia Wagenknecht, Qiang Zhang, Yu Ao Chen, Kai Chen, Jörg Schmiedmayer and Jian Wei Pan. Multistage entanglement swapping. Physical Review Letters, vol. 101, no. 8, 2008. (Cited on page 175.)

[Gordon et al. 2005] Karen Gordon, Veronica Fernandez, Gerald Buller, Ivan Rech, Sergio Cova and Paul Townsend. Quantum key distribution system clocked at 2 GHz. Optics express, vol. 13, no. 8, pages 3015-3020, 2005. (Cited on page 164.)

[Grangier et al. 2004] Philippe Grangier, Barry Sanders and Jelena Vuckovic. Focus on single photons on demand. New Journal of Physics, vol. 6, 2004. (Cited on page 127.)

[Grassani et al. 2015] Davide Grassani, Stefano Azzini, Marco Liscidini, Matteo Galli, Michael J. Strain, Marc Sorel, J. E. Sipe and Daniele Bajoni. Micrometer-scale integrated silicon source of time-energy entangled photons. Optica, vol. 2, no. 2, page 88, January 2015. (Cited on page 130.)

[Grice et al. 2001] W. Grice, a. URen and I. Walmsley. Eliminating frequency and spacetime correlations in multiphoton states. Physical Review A, vol. 64, no. 6, pages 1-7, 2001. (Cited on page 130.)

[Gross et al. 2012] Simon Gross, Martin Ams, Guido Palmer, Christopher T. Miese, Robert J. Williams, Graham D. Marshall, Alexander Fuerbach, David G. Lancaster, Heike Ebendorff-Heidepriem and Michael J. Withford. Ultrafast Laser Inscription in Soft Glasses: A Comparative Study of Athermal and Thermal Processing Regimes for Guided Wave Optics. International Journal of Applied Glass Science, vol. 3, no. 4, pages 332-348, December 2012. (Cited on page 140.)

[Gruber 1997] A. Gruber. Scanning Confocal Optical Microscopy and Magnetic Resonance on Single Defect Centers, 1997. (Cited on page 128.) 
[Guerreiro et al. 2013] T Guerreiro, A Martin, B Sanguinetti, N Bruno, H Zbinden and R T Thew. High efficiency coupling of photon pairs in practice. Optics express, vol. 21, no. 23, pages 27641-51, November 2013. (Cited on pages 156 and 167.)

[Halder et al. 2005] M. Halder, S. Tanzilli, H. de Riedmatten, A. Beveratos, H. Zbinden and N. Gisin. Photon-bunching measurement after two 25-km-long optical fibers. Physical Review A, vol. 71, no. 4, page 042335, April 2005. (Cited on page 184.)

[Halder et al. 2007] M Halder, A Beveratos, N Gisin, V Scarani, C Simon and H Zbinden. Entangling independent photons by time measurement. Nature Phys., vol. 3, no. 10, pages 692-695, 2007. (Cited on pages 121, 179, and 181.)

[Halder et al. 2008] Matthäus Halder, Alexios Beveratos, Robert T. Thew, Corentin Jorel, Hugo Zbinden and Nicolas Gisin. High coherence photon pair source for quantum communication. New Journal of Physics, vol. 10, 2008. (Cited on page 181.)

[Hamel et al. 2014] Deny R. Hamel, Lynden K. Shalm, Hannes Hübel, Aaron J. Miller, Francesco Marsili, Varun B. Verma, Richard P. Mirin, Sae Woo Nam, Kevin J. Resch and Thomas Jennewein. Direct generation of three-photon polarization entanglement. Nature Photonics, vol. 8, no. 10, pages 801-807, sep 2014. (Cited on page 65.)

[Hanbury Brown \& Twiss 1956] R. Hanbury Brown and R. Q. Twiss. A Test of a New Type of Stellar Interferometer on Sirius, 1956. (Cited on pages 5, 132, and 162.)

[Harada et al. 2008] Ken-ichi Harada, Hiroki Takesue, Hiroshi Fukuda, Tai Tsuchizawa, Toshifumi Watanabe, Koji Yamada, Yasuhiro Tokura and Sei-ichi Itabashi. Generation of high-purity entangled photon pairs using silicon wire waveguide. Optics express, vol. 16, no. 25, pages 20368-20373, 2008. (Cited on pages 65 and 130.)

[Harada et al. 2010] K.-I. Harada, H. Takesue, H. Fukuda, T. Tsuchizawa, T. Watanabe, K. Yamada, Y. Tokura and S.-I. Itabashi. Frequency and Polarization Characteristics of Correlated Photon-Pair Generation Using a Silicon Wire Waveguide. IEEE Journal of Selected Topics in Quantum Electronics, vol. 16, no. 1, pages 325-331, 2010. (Cited on page 134.) 
[Hensen et al. 2015] B. Hensen, H. Bernien, A. E. Dréau, A. Reiserer, N. Kalb, M. S. Blok, J. Ruitenberg, R. F. L. Vermeulen, R. N. Schouten, C. Abellán, W. Amaya, V. Pruneri, M. W. Mitchell, M. Markham, D. J. Twitchen, D. Elkouss, S. Wehner, T. H. Taminiau and R. Hanson. Loophole-free Bell inequality violation using electron spins separated by 1.3 kilometres. Nature, oct 2015. (Cited on pages 2 and 34.)

[Herbst et al. 2014] Thomas Herbst, Thomas Scheidl, Matthias Fink, Johannes Handsteiner, Bernhard Wittmann, Rupert Ursin and Anton Zeilinger. Teleportation of entanglement over $143 \mathrm{~km}$. arXiv:1403.0009, vol. 3, pages 1-7, 2014. (Cited on page 183.)

[Hoang et al. 2012] Thang B. Hoang, Johannes Beetz, Matthias Lermer, Leonardo Midolo, Martin Kamp, Sven Höfling and Andrea Fiore. Widely tunable, efficient on-chip single photon sources at telecommunication wavelengths, 2012. (Cited on page 128.)

[Holmes et al. 2014] Mark J. Holmes, Kihyun Choi, Satoshi Kako, Munetaka Arita and Yasuhiko Arakawa. Room-temperature triggered single photon emission from a IIInitride site-controlled nanowire quantum dot. Nano Letters, vol. 14, no. 2, pages 982-986, 2014. (Cited on page 128.)

[Hong et al. 1987] C K Hong, Z Y Ou and L Mandel. Measurement of subpicosecond time intervals between two photons by interference. Phys. Rev. Lett., vol. 59, no. 18, pages 2044-2046, 1987. (Cited on pages 68, 77, 153, and 184.)

[Humphreys et al. 2013] Peter C. Humphreys, Benjamin J. Metcalf, Justin B. Spring, Merritt Moore, Xian Min Jin, Marco Barbieri, W. Steven Kolthammer and Ian A. Walmsley. Linear optical quantum computing in a single spatial mode. Physical Review Letters, vol. 111, no. 15, 2013. (Cited on page 39.)

[Husko et al. 2013] Chad A Husko, Alex S Clark, Matthew J Collins, Alfredo De Rossi, Sylvain Combrié, Gaëlle Lehoucq, Isabella H Rey, Thomas F Krauss, Chunle Xiong and Benjamin J Eggleton. Multi-photon absorption limits to heralded single photon sources. Scientific reports, vol. 3, page 3087, January 2013. (Cited on page 130.) 
[Inagaki et al. 2013] Takahiro Inagaki, Nobuyuki Matsuda, Osamu Tadanaga, Masaki Asobe and Hiroki Takesue. Entanglement distribution over $300 \mathrm{~km}$ of fiber. Optics express, vol. 21, no. 20, pages 23241-9, 2013. (Cited on page 206.)

[Jain et al. 2011] Nitin Jain, Christoffer Wittmann, Lars Lydersen, Carlos Wiechers, Dominique Elser, Christoph Marquardt, Vadim Makarov and Gerd Leuchs. Device Calibration Impacts Security of Quantum Key Distribution. Physical Review Letters, vol. 107, no. 11, 2011. (Cited on page 44.)

[Jennewein et al. 2000] T. Jennewein, C. Simon, G. Weihs, H. Weinfurter and A. Zeilinger. Quantum Cryptography with Entangled Photons. Physical Review Letters, vol. 84, page 4729, 2000. (Cited on page 62.)

[Jennewein et al. 2002] Thomas Jennewein, Gregor Weihs, Jian-Wei Pan and Anton Zeilinger. Experimental nonlocality proof of quantum teleportation and entanglement swapping. Physical review letters, vol. 88, no. 1, page 017903, 2002. (Cited on pages 3 and 175.)

[Jiang \& Tomita 2007] Yun-Kun Jiang and Akihisa Tomita. The generation of polarizationentangled photon pairs using periodically poled lithium niobate waveguides in a fibre loop. Journal of Physics B: Atomic, Molecular and Optical Physics, vol. 40, no. 2, pages 437-443, 2007. (Cited on page 65.)

[Jin et al. 2014] Rui-Bo Jin, Ryosuke Shimizu, Isao Morohashi, Kentaro Wakui, Masahiro Takeoka, Shuro Izumi, Takahide Sakamoto, Mikio Fujiwara, Taro Yamashita, Shigehito Miki, Hirotaka Terai, Zhen Wang and Masahide Sasaki. Efficient generation of twin photons at telecom wavelengths with 2.5 GHz repetition-rate-tunable comb laser. Scientific reports, vol. 4, page 7468, January 2014. (Cited on pages 156 and 170.)

[Jin et al. 2015] Rui-Bo Jin, Masahiro Takeoka, Utako Takagi, Ryosuke Shimizu and Masahide Sasaki. Highly efficient entanglement swapping and teleportation at telecom wavelength. Scientific reports, vol. 5, page 9333, jan 2015. (Cited on pages 57, 175, and 180.) 
[Kaiser et al. 2012a] F. Kaiser, T. Coudreau, P. Milman, D. B. Ostrowsky and S. Tanzilli. Entanglement-Enabled Delayed-Choice Experiment. Science, vol. 338, no. 6107, pages 637-640, 2012. (Cited on pages 3, 62, and 131.)

[Kaiser et al. 2012b] F Kaiser, A Issautier, L A Ngah, O Danila, H Herrmann, W Sohler, A Martin and S Tanzilli. High-quality polarization entanglement state preparation and manipulation in standard telecommunication channels. New J. Phys., vol. 14, page 85015,2012 . (Cited on pages 5, 64, 67, 96, 102, and 206.)

[Kaiser et al. 2013] Florian Kaiser, Amandine Issautier, Lutfi A Ngah, Olivier Alibart, Anthony Martin and Sébastien Tanzilli. A versatile source of polarization entangled photons for quantum network applications. Laser Physics Letters, vol. 10, no. 4, page 045202, apr 2013. (Cited on pages 3, 5, 66, 67, 121, and 206.)

[Kaltenbaek et al. 2006] Rainer Kaltenbaek, Bibiane Blauensteiner, Marek Zukowski, Markus Aspelmeyer and Anton Zeilinger. Experimental interference of independent photons. Physical Review Letters, vol. 96, no. 24, 2006. (Cited on page 180.)

[Kaltenbaek et al. 2009] Rainer Kaltenbaek, Robert Prevedel, Markus Aspelmeyer and Anton Zeilinger. High-fidelity entanglement swapping with fully independent sources. Physical Review A - Atomic, Molecular, and Optical Physics, vol. 79, no. 4, 2009. (Cited on pages 57, 179, and 180.)

[Kaneda et al. 2015] Fumihiro Kaneda, Bradley G. Christensen, Jia Jun Wong, Kevin T. McCusker, Hee Su Park and Paul G. Kwiat. A Time-Multiplexed Heralded SinglePhoton Source. July 2015. (Cited on page 137.)

[Kashin et al. 2011] O. Kashin, M. Homann, V. Matusevich, F. Setzpfandt, T. Pertsch and $\mathrm{R}$. Kowarschik. Change of the refractive index in PPLN waveguides due to the photorefractive effect. Applied Physics B, vol. 104, no. 3, pages 547-551, August 2011. (Cited on page 191.)

[Kiess et al. 1993] T. E. Kiess, Yanhua Shih, Alexander V. Sergienko and Carroll O. Alley. Einstein-Podolsky-Rosen-Bohm Experiment Using Pairs of Light Quanta Produced 
by Type-II Parametric Down-Conversion. Physical Review Letters, vol. 71, no. 24, pages 3893-3897, 1993. (Cited on pages 62 and 68.)

[Kim et al. 2000] Yoon-Ho Kim, Sergei P. Kulik and Yanhua Shih. High-intensity pulsed source of space-time and polarization double-entangled photon pairs. Physical Review A, vol. 62 , no. 1, pages 4-7, 2000. (Cited on pages 63 and 65.)

[Knill et al. 2001] E Knill, R Laflamme and G J Milburn. A scheme for efficient quantum computation with linear optics. Nature, vol. 409, no. 6816, pages 46-52, 2001. (Cited on pages 2 and 126.)

[Krapick et al. 2013] S Krapick, H Herrmann, V Quiring, B Brecht, H Suche and Ch Silberhorn. An efficient integrated two-color source for heralded single photons. New Journal of Physics, vol. 15, no. 3, page 033010, March 2013. (Cited on pages 2, 129, 156,168 , and 170.)

[Kuklewicz et al. 2006] C E Kuklewicz, F N C Wong and J H Shapiro. Time-bin-modulated biphotons from cavity-enhanced down-conversion. Phys. Rev. Lett., vol. 97, no. 22, page 223601, 2006. (Cited on pages 3, 62, 68, and 121.)

[Kurtsiefer et al. 2000] Christian Kurtsiefer, Sonja Mayer, Patrick Zarda and Harald Weinfurter. Stable solid-state source of single photons. Physical Review Letters, vol. 85, no. 2, pages 290-293, 2000. (Cited on page 127.)

[Kwiat et al. 1993] P G Kwiat, A M Steinberg and R Y Chiao. High-visibility interference in a $\{B\}$ ell-inequality experiment for energy and time. Phys. Rev. A, vol. 47, no. 4, pages R2472-R2475, 1993. (Cited on pages 66 and 109.)

[Kwiat et al. 1995] P G Kwiat, K Mattle, H Weinfurter, A Zeilinger, A V Sergienko and Y Shih. New \{High-Intensity\} Source of \{Polarization-Entangled\} Photon Pairs. Phys. Rev. Lett., vol. 75, no. 24, pages 4337-4341, 1995. (Cited on pages 63 and 131.)

[Kwiat et al. 1999] P G Kwiat, E Waks, A G White, I Appelbaum and P H Eberhard. Ultrabright source of polarization-entangled photons. Phys. Rev. A, vol. 60, no. 2, pages R773-R776, 1999. (Cited on page 63.) 
[Lamas-Linares et al. 2002] Antia Antía Lamas-Linares, Christoph Simon, D, J.C. John C. J.C. Howell and Dik Bouwmeester. Experimental Quantum Cloning of Single Photons. Science, vol. 296, no. 1, page 4, 2002. (Cited on page 25.)

[Lea 2002] Measuring the Orbital Angular Momentum of a Single Photon. Physical Review Letters, vol. 88, no. 25, page 257901, 2002. (Cited on page 39.)

[Lee et al. 2006] K F Lee, J Chen, C Liang, X Li, P L Voss and P Kumar. Generation of high-purity telecom-band entangled photon pairs in dispersion-shifted fiber. Opt. Lett., vol. 31, no. 12, pages 1905-1907, 2006. (Cited on page 96.)

[Legero et al. 2003] T. Legero, T. Wilk, A. Kuhn and G. Rempe. Time-resolved two-photon quantum interference. Applied Physics B: Lasers and Optics, vol. 77, no. 8, pages 797-802, December 2003. (Cited on page 184.)

[Li et al. 2004] Xiaoying Li, Jun Chen, Paul Voss, Jay Sharping and Prem Kumar. All-fiber photon-pair source for quantum communications: Improved generation of correlated photons. Optics Express, vol. 12, no. 16, page 3737, August 2004. (Cited on pages 65 and 129.)

[Li et al. 2005] X Y Li, P L Voss, J E Sharping and P Kumar. Optical-fiber source of polarization-entangled photons in the $1550 \mathrm{~nm}$ telecom band. Phys. $\backslash$ Rev. $\backslash$ Lett., vol. 94 , no. 5, page 53601, 2005. (Cited on page 65.)

[Little et al. 2008] Douglas J Little, Martin Ams, Peter Dekker, Graham D Marshall, Judith M Dawes and Michael J Withford. Femtosecond laser modification of fused silica: the effect of writing polarization on Si-O ring structure. Optics express, vol. 16, no. 24, pages 20029-20037, 2008. (Cited on page 140.)

[Lo et al. 2005] H K. Lo, X Ma and K Chen. Decoy state quantum key distribution. Physical Review Letters, vol. 94, no. 23, page 230504, 2005. (Cited on page 2.)

[Lo et al. 2012] Hoi-Kwong Lo, Marcos Curty and Bing Qi. Measurement-DeviceIndependent Quantum Key Distribution, 2012. (Cited on page 44.)

[Lütkenhaus 2000] N Lütkenhaus. Security against individual attacks for realistic quantum key distribution. Phys. Rev. A, vol. 61, no. 5, page 52304, 2000. (Cited on page 47.) 
[Lv et al. 2013] Ning Lv, Wei Zhang, Yuan Guo, Qiang Zhou, Yidong Huang and Jiangde Peng. 1.5 um polarization entanglement generation based on birefringence in silicon wire waveguides. Opt. Lett., vol. 38, no. 15, pages 2873-2876, 2013. (Cited on page 65.)

[Lydersen et al. 2010a] Lars Lydersen, Carlos Wiechers, Christoffer Wittmann, Dominique Elser, Johannes Skaar and Vadim Makarov. Hacking commercial quantum cryptography systems by tailored bright illumination. Nature Photonics, vol. 4, no. 10, pages 686-689, August 2010. (Cited on pages 43 and 44.)

[Lydersen et al. 2010b] Lars Lydersen, Carlos Wiechers, Christoffer Wittmann, Dominique Elser, Johannes Skaar and Vadim Makarov. Thermal blinding of gated detectors in quantum cryptography. Optics express, vol. 18, no. 26, pages 27938-27954, 2010. (Cited on page 44.)

[Lydersen et al. 2011] Lars Lydersen, Nitin Jain, Christoffer Wittmann, Ø ystein Marø y, Johannes Skaar, Christoph Marquardt, Vadim Makarov and Gerd Leuchs. Superlinear threshold detectors in quantum cryptography. Physical Review A - Atomic, Molecular, and Optical Physics, vol. 84, no. 3, 2011. (Cited on page 44.)

[Ma et al. 2012a] X Ma, T Herbst, T Scheidl, W Daqing, S Kropatschek, W Naylor, B Wittmann, A Mech, J Kofler, E Anisimova, V Makarov, T Jennewein, R Ursin and A Zeilinger. Quantum teleportation over 143 kilometres using active feed-forward. Nature, vol. 489, pages 269-273, 2012. (Cited on page 56.)

[Ma et al. 2012b] Xiao-song Ma, Stefan Zotter, Johannes Kofler, Rupert Ursin, Thomas Jennewein, Časlav Brukner and Anton Zeilinger. Experimental delayed-choice entanglement swapping, 2012. (Cited on page 175.)

[Mair et al. 2001] A Mair, A Vaziri, G Weihs and A Zeilinger. Entanglement of the orbital angular momentum states of photons. Nature, vol. 412, no. 6844, pages 313-316, 2001. (Cited on page 39.)

[Makarov 2009] Vadim Makarov. Controlling passively quenched single photon detectors by bright light. New Journal of Physics, vol. 11, 2009. (Cited on page 44.) 
[Maker et al. 1962] P. D. Maker, R. W. Terhune, M. Nisenoff and C. M. Savage. Effects of Dispersion and Focusing on the Production of Optical Harmonics. Physical Review Letters, vol. 8, no. 1, pages 21-22, jan 1962. (Cited on page 68.)

[Marcikic et al. 2003] I Marcikic, H de Riedmatten, W Tittel, H Zbinden and N Gisin. Longdistance teleportation of qubits at telecommunication wavelengths. Nature (London)\}, vol. 421, pages 509-513, 2003. (Cited on page 56.)

[Marseglia et al. 2011] L. Marseglia, J. P. Hadden, A. C. Stanley-Clarke, J. P. Harrison, B. Patton, Y. L D Ho, B. Naydenov, F. Jelezko, J. Meijer, P. R. Dolan, J. M. Smith, J. G. Rarity and J. L. O'brien. Nanofabricated solid immersion lenses registered to single emitters in diamond. Applied Physics Letters, vol. 98, no. 13, 2011. (Cited on page 128.)

[Marsili et al. 2013] F. Marsili, V. B. Verma, J. a. Stern, S. Harrington, a. E. Lita, T. Gerrits, I. Vayshenker, B. Baek, M. D. Shaw, R. P. Mirin and S. W. Nam. Detecting Single Infrared Photons with 93\% System Efficiency. Nature Photonics, vol. 7, no. 3, pages 210-214, February 2013. (Cited on page 170.)

[Martin et al. 2009] a Martin, V Cristofori, P Aboussouan, H Herrmann, W Sohler, D B Ostrowsky, O Alibart and S Tanzilli. Integrated optical source of polarization entangled photons at $1310 \mathrm{~nm}$. Optics express, vol. 17, no. 2, pages 1033-1041, 2009. (Cited on pages 64 and 130.)

[Martin et al. 2010] A Martin, A Issautier, H Herrmann, W Sohler, D B Ostrowsky, O Alibart and S Tanzilli. A polarization entangled photon-pair source based on a $\{$ type-II\} $\{P P L N\}$ waveguide emitting at a telecom wavelength. New J. Phys., vol. 12, no. 10, page 103005, 2010. (Cited on pages 64, 74, 76, and 96.)

[Massar et al. 2002] Serge Massar, Stefano Pironio, Jérémie Roland and Bernard Gisin. Bell inequalities resistant to detector inefficiency. Physical Review A, vol. 66, no. 5, page 12, 2002. (Cited on page 43.)

[Massar 2002] Serge Massar. Non locality, closing the detection loophole and communication complexity. Physical Review A, vol. 65, page 032121, 2002. (Cited on page 43.) 
[Matthews et al. 2013] Jonathan C F Matthews, Konstantinos Poulios, Jasmin D A Meinecke, Alberto Politi, Alberto Peruzzo, Nur Ismail, Kerstin Wörhoff, Mark G Thompson and Jeremy L O'Brien. Observing fermionic statistics with photons in arbitrary processes. Scientific reports, vol. 3, page 1539, jan 2013. (Cited on page 72.)

[Mattle et al. 1996] Klaus Mattle, Harald Weinfurter, Paul G. Kwiat and Anton Zeilinger. Dense Coding in Experimental Quantum Communication. Physical Review Letters, vol. 76, no. 25, pages 4656-4659, 1996. (Cited on page 62.)

[McMillan et al. 2013] a R McMillan, L Labonté, a S Clark, B Bell, O Alibart, a Martin, W J Wadsworth, S Tanzilli and J G Rarity. Two-photon interference between disparate sources for quantum networking. Scientific reports, vol. 3, page 2032, 2013. (Cited on page 179.)

[Meany et al. 2012] Thomas Meany, Michael Delanty, Simon Gross, Graham D Marshall, M J Steel and Michael J Withford. Non-classical interference in integrated 3D multiports. Opt. Express, vol. 20, no. 24, pages 26895-26905, 2012. (Cited on pages 142 and 156.)

[Meany et al. 2014] Thomas Meany, Lutfi A. Ngah, Matthew J. Collins, Alex S. Clark, Robert J. Williams, Benjamin J. Eggleton, M. J. Steel, Michael J. Withford, Olivier Alibart and Sébastien Tanzilli. Hybrid photonic circuit for multiplexed heralded single photons, 2014. (Cited on pages 6 and 207.)

[Medic et al. 2010] M Medic, J B Altepeter, M A Hall, M Patel and P Kumar. Fiber-based telecommunication-band source of degenerate entangled photons. Opt. Lett., vol. 35, no. 6, pages 802-804, 2010. (Cited on page 96.)

[Mendoza et al. 2015] Gabriel J. Mendoza, Raffaele Santagati, Jack Munns, Elizabeth Hemsley, Mateusz Piekarek, Enrique Martin-Lopez, Graham D. Marshall, Damien Bonneau, Mark G. Thompson and Jeremy L. O'Brien. Active Temporal Multiplexing of Photons. March 2015. (Cited on page 137.) 
[Migdall et al. 2002] A. Migdall, D. Branning and S. Castelletto. Tailoring single-photon and multiphoton probabilities of a single-photon on-demand source, 2002. (Cited on pages 136 and 156.)

[Mizuochi et al. 2012] N. Mizuochi, T. Makino, H. Kato, D. Takeuchi, M. Ogura, H. Okushi, M. Nothaft, P. Neumann, A. Gali, F. Jelezko, J. Wrachtrup and S. Yamasaki. Electrically driven single-photon source at room temperature in diamond, 2012. (Cited on pages 2 and 128.)

[Morris et al. 2014] Oliver J. Morris, Robert J.A. Francis-Jones, Keith G. Wilcox, Anne C. Tropper and Peter J. Mosley. Photon-pair generation in photonic crystal fibre with a 1.5GHz modelocked VECSEL. Optics Communications, vol. 327, pages 39-44, September 2014. (Cited on page 157.)

[Mosley et al. 2008a] P. J. Mosley, J. S. Lundeen, B. J. Smith and I. A. Walmsley. Conditional preparation of single photons using parametric downconversion: A recipe for purity. New Journal of Physics, vol. 10, 2008. (Cited on pages 130, 145, 156, and 169.)

[Mosley et al. 2008b] Peter J. Mosley, Jeff S. Lundeen, Brian J. Smith, Piotr Wasylczyk, Alfred B. U'Ren, Christine Silberhorn and Ian A. Walmsley. Heralded generation of ultrafast single photons in pure quantum states. Physical Review Letters, vol. 100, no. 13, 2008. (Cited on pages 130, 145, and 169.)

[Nagali et al. 2009] Eleonora Nagali, Linda Sansoni, Fabio Sciarrino, Francesco De Martini, Lorenzo Marrucci, Bruno Piccirillo, Ebrahim Karimi and Enrico Santamato. Optimal quantum cloning of orbital angular momentum photon qubits through HongOu-Mandel coalescence. Nature Photonics, vol. 3, no. 12, pages 720-723, 2009. (Cited on page 39.)

[Neergaard-Nielsen et al. 2007] Jonas S Neergaard-Nielsen, Bo Melholt Nielsen, Hiroki Takahashi, Arnt Inge Vistnes and Eugene S Polzik. High purity bright single photon source. Optics Express, vol. 15, no. 13, pages 7940-7949, 2007. (Cited on page 68.) 
[Nemoto \& Munro 2004] Kae Nemoto and W. J. Munro. Nearly deterministic linear optical controlled-NOT gate. Physical Review Letters, vol. 93, no. 25, 2004. (Cited on page 126.)

[Ngah et al. 2015] Lutfi Arif Ngah, Olivier Alibart, Laurent Labonté, Virginia D'Auria and Sébastien Tanzilli. Ultra-fast heralded single photon source based on telecom technology. Laser \& Photonics Reviews, vol. 9, no. 2, pages L1-L5, March 2015. (Cited on pages 2,6 , and 207.)

[Nicolas et al. 2014] a. Nicolas, L. Veissier, L. Giner, E. Giacobino, D. Maxein and J. Laurat. A quantum memory for orbital angular momentum photonic qubits. Nature Photonics, vol. 8, no. January, pages 1-5, 2014. (Cited on page 39.)

[Nie et al. 2015] You-Qi Nie, Leilei Huang, Yang Liu, Frank Payne, Jun Zhang and JianWei Pan. The generation of 68 Gbps quantum random number by measuring laser phase fluctuations. The Review of scientific instruments, vol. 86, no. 6, page 063105, June 2015. (Cited on page 45.)

[O'Brien et al. 2003] J L O'Brien, G J Pryde, A G White, T C Ralph and D Branning. Demonstration of an all-optical quantum controlled-NOT gate. Nature, vol. 426, no. 6964, pages 264-7, November 2003. (Cited on page 126.)

[Olislager et al. 2010a] L Olislager, J Cussey, A T Nguyen, P Emplit, S Massar, J.-M. Merolla and K P Huy. Frequency-bin entangled photons. Phys. Rev. A, vol. 82, no. 1, page 13804, 2010. (Cited on page 39.)

[Olislager et al. 2010b] L Olislager, J Cussey, A T Nguyen, P Emplit, S Massar, J.-M. Merolla and K P Huy. Manipulating Frequency Entangled Photons, volume 36. Springer Berlin Heidelberg, Berlin, Heidelberg, 2010. (Cited on page 39.)

[Olislager et al. 2011] L Olislager, I Mbodji, E Woodhead, J Cussey, L Furfaro, P Emplit, S Massar, K P Huy and J.-M. Merolla. Reliable and efficient control of two-photon interference in the frequency domain. arXiv:1107.5519, 2011. (Cited on page 39.) 
[Ou \& Mandel 1988] Z. Y. Ou and L. Mandel. Violation of Bell's Inequality and Classical Probability in a Two-Photon Correlation Experiment. Physical Review Letters, vol. 61 , no. 1 , pages $50-53$, jul 1988. (Cited on page 68.)

[Owens et al. 2011] J. O. Owens, M. A. Broome, D. N. Biggerstaff, M. E. Goggin, A. Fedrizzi, T. Linjordet, M. Ams, G. D. Marshall, J. Twamley, M. J. Withford and A. G. White. Two-photon quantum walks in an elliptical direct-write waveguide array. New Journal of Physics, vol. 13, 2011. (Cited on page 140.)

[Pan et al. 1998] Jian-Wei Pan, Dik Bouwmeester, Harald Weinfurter and Anton Zeilinger. Experimental Entanglement Swapping: Entangling Photons That Never Interacted, 1998. (Cited on pages 57 and 180.)

[Patel et al. 2014] K. a. Patel, J. F. Dynes, M. Lucamarini, I. Choi, a. W. Sharpe, Z. L. Yuan, R. V. Penty and a. J. Shields. Quantum key distribution for $10 \mathrm{~Gb} / \mathrm{s}$ dense wavelength division multiplexing networks. Applied Physics Letters, vol. 104, no. 5, feb 2014. (Cited on pages 71 and 102.)

[Pearle 1970] P M Pearle. Hidden-Variable Example Based upon Data Rejection. Phys. Rev. D, vol. 2, pages 1418-1425, 1970. (Cited on page 34.)

[Pelton et al. 2002] Matthew Pelton, Charles Santori, Jelena Vucković, Bingyang Zhang, Glenn S. Solomon, Jocelyn Plant and Yoshihisa Yamamoto. Efficient Source of Single Photons: A Single Quantum Dot in a Micropost Microcavity. Physical Review Letters, vol. 89, no. 23, page 233602, November 2002. (Cited on page 127.)

[Peruzzo et al. 2012] a. Peruzzo, P. Shadbolt, N. Brunner, S. Popescu and J. L. O'Brien. A Quantum Delayed-Choice Experiment. Science, vol. 338, no. 6107, pages 634-637, 2012. (Cited on page 3.)

[Piro et al. 2009] N Piro, A Haase, M W Mitchell and J Eschner. An entangled photon source for resonant single-photon-single-atom interaction. J. Phys. B: At. Mol. Opt. Phys., vol. 42, page 114002, June 2009. (Cited on pages 3, 76, 96, 104, and 121.) 
[Polyakov \& Migdall 2009] Sergey V. Polyakov and Alan L. Migdall. Quantum radiometry. Journal of Modern Optics, vol. 56, no. 9, pages 1045-1052, May 2009. (Cited on pages 2 and 126.)

[Pomarico et al. 2009] E Pomarico, B Sanguinetti, N Gisin, R Thew, H Zbinden, G Schreiber, A Thomas and W Sohler. Waveguide-based $\{O P O\}$ source of entangled photon pairs. New J. Phys., vol. 11, page 113042, 2009. (Cited on pages 111 and 121.)

[Pomarico et al. 2012] Enrico Pomarico, Bruno Sanguinetti, Thiago Guerreiro, Rob Thew and Hugo Zbinden. MHz rate and efficient synchronous heralding of single photons at telecom wavelengths, 2012. (Cited on pages 2, 129, 156, 169, and 170.)

[Press et al. 2007] David Press, Stephan Götzinger, Stephan Reitzenstein, Carolin Hofmann, Andreas Löffler, Martin Kamp, Alfred Forchel and Yoshihisa Yamamoto. Photon antibunching from a single quantum-dot-microcavity system in the strong coupling regime. Physical Review Letters, vol. 98, no. 11, 2007. (Cited on pages 2 and 127.)

[Pütz et al. 2015] Gilles Pütz, Djeylan Aktas, Anthony Martin, Bruno Fedrici, Sébastien Tanzilli and Nicolas Gisin. Quantum nonlocality with arbitrary limited detection efficiency. page 5, sep 2015. (Cited on page 43.)

[Qi et al. 2007] Bing Qi, Chi-Hang Fred Fung, Hoi-Kwong Lo and Xiongfeng Ma. Time-shift attack in practical quantum cryptosystems. Quantum Information \& Computation, vol. 7, no. 1, pages 73-82, January 2007. (Cited on page 44.)

[Qi et al. 2010a] Bing Qi, Yue-Meng Chi, Hoi-Kwong Lo and Li Qian. High-speed quantum random number generation by measuring phase noise of a single-mode laser. Optics letters, vol. 35, no. 3, pages 312-314, 2010. (Cited on page 45.)

[Qi et al. 2010b] Bing Qi, Wen Zhu, Li Qian and Hoi-Kwong Lo. Feasibility of quantum key distribution through dense wavelength division multiplexing network. New Journal of Physics, vol. 12, no. 10, page 18, 2010. (Cited on pages 71 and 102.) 
[Ramelow et al. 2013] Sven Ramelow, Alexandra Mech, Marissa Giustina, Simon Gröblacher, Witlef Wieczorek, Jörn Beyer, Adriana Lita, Brice Calkins, Thomas Gerrits, Sae Woo Nam, Anton Zeilinger and Rupert Ursin. Highly efficient heralding of entangled single photons. Optics express, vol. 21, no. 6, pages 6707-17, March 2013. (Cited on pages 39, 156, 167, and 170.)

[Rangarajan et al. 2009] Radhika Rangarajan, Michael Goggin and Paul Kwiat. Optimizing type-I polarization-entangled photons. Optics express, vol. 17, no. 21, pages 18920 18933, 2009. (Cited on page 64.)

[Rarity \& Tapster 1989] J. G. Rarity and P. R. Tapster. Fourth-order interference in parametric downconversion, 1989. (Cited on page 184.)

[Rarity et al. 1987] J. G. Rarity, P. R. Tapster and E. Jakeman. Observation of subpoissonian light in parametric downconversion. Optics Communications, vol. 62, no. 3, pages 201-206, 1987. (Cited on page 129.)

[Rarity et al. 1994] J.G. Rarity, P.C.M. Owens and P.R. Tapster. Quantum Randomnumber Generation and Key Sharing. Journal of Modern Optics, vol. 41, no. 12, pages 2435-2444, dec 1994. (Cited on page 43.)

[Rarity et al. 2005] J Rarity, J Fulconis, J Duligall, W Wadsworth and P Russell. Photonic crystal fiber source of correlated photon pairs. Optics express, vol. 13, no. 2, pages 534-544, 2005. (Cited on pages 65 and 129.)

[Rarity 1995] J. G. Rarity. Interference of Single Photons from Separate Sourcesa. Annals of the New York Academy of Sciences, vol. 755, no. 1, pages 624-631, April 1995. (Cited on page 184.)

[Reim et al. 2010] K. F. Reim, J. Nunn, V. O. Lorenz, B. J. Sussman, K. C. Lee, N. K. Langford, D. Jaksch and I. A. Walmsley. Towards high-speed optical quantum memories. Nature Photonics, vol. 4, no. 4, pages 218-221, 2010. (Cited on page 104.)

[Saglamyurek et al. 2011] E Saglamyurek, N Sinclair, J Jin, J A Slater, D Oblak, F Bussieres, M George, R Ricken, W Sohler and W Tittel. Broadband waveguide 
quantum memory for entangled photons. Nature, vol. 469, no. 7331, pages 512-515, 2011. (Cited on page 104.)

[Sanaka et al. 2001] K. Sanaka, K. Kawahara and T. Kuga. New high-efficiency source of photon pairs for engineering quantum entanglement. Physical Review Letters, vol. 86, no. 24, pages 5620-5623, 2001. (Cited on pages 64 and 69.)

[Sanaka et al. 2002] Kaoru Sanaka, Karin Kawahara and Takahiro Kuga. Experimental probabilistic manipulation of down-converted photon pairs using unbalanced interferometers. Physical Review A, vol. 66, no. 4, page 040301, 2002. (Cited on pages 106 and 111.)

[Sangouard et al. 2011] Nicolas Sangouard, Christoph Simon, Hugues De Riedmatten and Nicolas Gisin. Quantum repeaters based on atomic ensembles and linear optics. Reviews of Modern Physics, vol. 83, no. 1, pages 33-80, 2011. (Cited on page 66.)

[Santori et al. 2001] Charles Santori, Matthew Pelton, Glenn Solomon, Yseulte Dale and Yoshihisa Yamamoto. Triggered single photons from a quantum dot. Physical Review Letters, vol. 86, no. 8, pages 1502-1505, 2001. (Cited on pages 2 and 127.)

[Scarani et al. 2004a] Valerio Scarani, G Ribordy A. Acin, Nicolas Gisin, Antonio Acín and Grégoire Ribordy. Quantum Cryptography Protocols Robust against Photon Number Splitting Attacks for Weak Laser Pulse Implementations. Physical review letters, vol. 92 , no. 5 , page 057901,2004 . (Cited on page 2.)

[Scarani et al. 2004b] Valerio Scarani, Nicolas Gisin and Sandu Popescu. Proposal for energy-time entanglement of quasiparticles in a solid-state device. Physical review letters, vol. 92, no. 16, page 167901, apr 2004. (Cited on page 2.)

[Scarani et al. 2009] Valerio Scarani, Helle Bechmann-Pasquinucci, Nicolas J. Cerf, Miloslav Dušek, Norbert Lütkenhaus and Momtchil Peev. The security of practical quantum key distribution. Reviews of Modern Physics, vol. 81, no. 3, pages 1301-1350, 2009. (Cited on pages 2 and 126.)

[Sekatski et al. 2012] P Sekatski, N Sangouard, F Bussières, C Clausen, N Gisin and H Zbinden. Detector imperfections in photon-pair source characterization. Journal 
of Physics B: Atomic, Molecular and Optical Physics, vol. 45, no. 12, page 124016, June 2012. (Cited on pages 168 and 169.)

[Sharping et al. 2006] Jay E Sharping, Kim F Lee, Mark A Foster, Amy C Turner, Bradley S Schmidt, Michal Lipson, Alexander L Gaeta and Prem Kumar. Generation of correlated photons in nanoscale silicon waveguides. Optics express, vol. 14, no. 25, pages 12388-12393, 2006. (Cited on pages 65 and 130.)

[Shi \& Tomita 2004] Bao-Sen Shi and Akihisa Tomita. Generation of a pulsed polarization entangled photon pair using a Sagnac interferometer. Physical Review A, vol. 69, no. 1, page 013803, 2004. (Cited on page 63.)

[Shih \& Alley 1988] Yanhua Shih and Carroll O. Alley. New Type of Einstein-PodolskyRosen-Bohm Experiment Using Pairs of Light Quanta Produced by Optical Parametric Down Conversion. Physical Review Letters, vol. 61, no. 26, pages 2921-2924, 1988. (Cited on pages 68 and 109.)

[Singh 2000] S Singh. The Code Book: The Science of Secrecy from Ancient Egypt to Quantum Cryptography. Anchor, 2000. (Cited on page 16.)

[Sohler 1989] W Sohler. Integrated optics in $\{$ LiNbO\}_3. Thin Solid Films, vol. 175, pages 191-200, 1989. (Cited on page 70.)

[Specht et al. 2011] H P Specht, C Nolleke, A Reiserer, M Uphoff, E Figueroa, S Ritter and G Rempe. A single-atom quantum memory. Nature, vol. 473, no. 7346, pages 190-193, 2011. (Cited on page 104.)

[Steinlechner et al. 2012] Fabian Steinlechner, Pavel Trojek, Marc Jofre, Henning Weier, Daniel Perez, Thomas Jennewein, Rupert Ursin, John Rarity, Morgan W. Mitchell, Juan P. Torres, Harald Weinfurter and Valerio Pruneri. A high-brightness source of polarization-entangled photons optimized for applications in free space. Optics Express, vol. 20, no. 9, page 9640, 2012. (Cited on page 64.)

[Strekalov et al. 1996] D. Strekalov, T. Pittman, a. Sergienko, Y. Shih and P. Kwiat. Postselection-free energy-time entanglement. Physical Review A, vol. 54, no. 1, pages R1-R4, 1996. (Cited on page 62.) 
[Stucki et al. 2009] Damien Stucki, Claudio Barreiro, Sylvain Fasel, Jean-Daniel Gautier, Olivier Gay, Nicolas Gisin, Rob Thew, Yann Thoma, Patrick Trinkler, Fabien Vannel and Hugo Zbinden. Continuous high speed coherent one-way quantum key distribution. Optics express, vol. 17, no. 16, pages 13326-13334, 2009. (Cited on page 2.)

[Suhara et al. 2007] T Suhara, H Okabe and M Fujimura. Generation of pol-arizationentangled photons by $\{$ Type-II\} quasi-phase-matched waveguide non $\backslash$-linear-optic device. \{IEEE\} Photon. Technol. Lett., vol. 19, no. 14, pages 1093-1095, 2007. (Cited on pages 64 and 96.)

[Takesue \& Inoue 2004] Hiroki Takesue and Kyo Inoue. Generation of polarizationentangled photon pairs and violation of Bell's inequality using spontaneous four-wave mixing in a fiber loop. Physical Review A - Atomic, Molecular, and Optical Physics, vol. 70, no. 3, 2004. (Cited on pages 65 and 129.)

[Takesue \& Inoue 2005] H Takesue and K Inoue. 1.5- $\mu m$ band quantum-correlated photon pair generation in dispersion-shifted fiber: suppression of noise photons by cooling fiber. Opt. Express, vol. 13, no. 20, pages 7832-7839, 2005. (Cited on page 136.)

[Takesue \& Miquel 2009] Hiroki Takesue and Benjamin Miquel. Entanglement swapping using telecom-band photons generated in fibers. Optics express, vol. 17, no. 13, pages 10748-10756, 2009. (Cited on pages 57, 179, 180, and 182.)

[Takesue et al. 2005] H Takesue, K Inoue, O Tadanaga, Y Nishida and M Asobe. Generation of pulsed polarization-entangled photon pairs in a 1.55- $\mu \mathrm{m}$ band with a periodically poled lithium niobate waveguide and anorthogonal polarization delay circuit. Opt. Lett., vol. 30, no. 3, pages 293-295, 2005. (Cited on pages 65, 106, and 111.)

[Takesue et al. 2007a] Hiroki Takesue, Sae Woo Nam, Qiang Zhang, Robert H. Hadfield, Toshimori Honjo, Kiyoshi Tamaki and Yoshihisa Yamamoto. Quantum key distribution over a 40-dB channel loss using superconducting single-photon detectors. Nature Photonics, vol. 1, no. 6, pages 343-348, 2007. (Cited on page 164.)

[Takesue et al. 2007b] Hiroki Takesue, Yasuhiro Tokura, Hiroshi Fukuda, Tai Tsuchizawa, Toshifumi Watanabe, Koji Yamada and Sei-ichi Itabashi. Entanglement generation 
using silicon wire waveguide. Applied Physics Letters, vol. 91, no. 20, page 201108, November 2007. (Cited on page 130.)

[Takesue et al. 2008] Hiroki Takesue, Hiroshi Fukuda, Tai Tsuchizawa, Toshifumi Watanabe, Koji Yamada, Yasuhiro Tokura and Sei-ichi Itabashi. Generation of polarization entangled photon pairs using silicon wire waveguide. Optics Express, vol. 16, no. 8, page 5721, 2008. (Cited on page 65.)

[Takesue et al. 2010] Hiroki Takesue, Ken-Ichi Harada, Hiroshi Fukuda, Tai Tsuchizawa, Toshifumi Watanabe, Koji Yamada, Yasuhiro Tokura and Sei-Ichi Itabashi. Entanglement generation using silicon photonic wire waveguide. Journal of nanoscience and nanotechnology, vol. 10, no. 3, pages 1814-1818, 2010. (Cited on page 65.)

[Takesue et al. 2015] Hiroki Takesue, Shellee D. Dyer, Martin J. Stevens, Varun Verma, Richard P. Mirin and Sae Woo Nam. Quantum teleportation over $100 \mathrm{~km}$ of fiber using highly efficient superconducting nanowire single-photon detectors. Optica, vol. 2, no. 10, page 832, September 2015. (Cited on page 56.)

[Tamarat et al. 2006] Ph Tamarat, T. Gaebel, J. R. Rabeau, M. Khan, A. D. Greentree, H. Wilson, L. C L Hollenberg, S. Prawer, P. Hemmer, F. Jelezko and J. Wrachtrup. Stark shift control of single optical centers in diamond. Physical Review Letters, vol. 97, no. 8, 2006. (Cited on pages 2 and 128.)

[Tanzilli et al. 2001] S Tanzilli, H de Riedmatten, W Tittel, H Zbinden, P Baldi, M P De Micheli, D B Ostrowsky and N Gisin. Highly efficient photon-pair source using a Periodically Poled Lithium Niobate waveguide. Electron. Lett., vol. 37, pages 26-28, 2001. (Cited on pages 64, 69, and 94.)

[Tanzilli et al. 2002] S. Tanzilli, W. Tittel, H. De Riedmatten, H. Zbinden, P. Baldi, M. Demicheli, D. B. Ostrowsky and N. Gisin. PPLN waveguide for quantum communication. In European Physical Journal D, volume 18, pages 155-160, 2002. (Cited on pages $64,66,69,94,111,117,130,131$, and 159.) 
[Thew et al. 2002] R T Thew, S Tanzilli, W Tittel, H Zbinden and N Gisin. Experimental investigation of the robustness of partially entangled qubits over $11 \mathrm{~km}$. Phys. Rev. A, vol. 66, no. 6, page 62304, 2002. (Cited on page 66.)

[Tillmann et al. 2013] Max Tillmann, Borivoje Dakić, René Heilmann, Stefan Nolte, Alexander Szameit and Philip Walther. Experimental boson sampling. Nature Photonics, vol. 7, no. 7, pages 540-544, 2013. (Cited on pages 2 and 126.)

[Townsend et al. 1993] P.D. Townsend, J.G. Rarity and P.R. Tapster. Single photon interference in $10 \mathrm{~km}$ long optical fibre interferometer, 1993. (Cited on page 37.)

[Valivarthi et al. 2015] Raju Valivarthi, Itzel Lucio-Martinez, Philip Chan, Allison Rubenok, Caleb John, Daniel Korchinski, Cooper Duffin, Francesco Marsili, Varun Verma, Mathew D. Shaw, Jeffrey A. Stern, Sae Woo Nam, Daniel Oblak, Qiang Zhou, Joshua A. Slater and Wolfgang Tittel. Measurement-device-independent quantum key distribution: from idea towards application. Journal of Modern Optics, vol. 62, no. 14, pages 1141-1150, March 2015. (Cited on page 44.)

[van Houwelingen et al. 2006] J A W van Houwelingen, N Brunner, A Beveratos, H Zbinden and N Gisin. Quantum Teleportation with a Three-Bell-State Analyzer. Phys. Rev. Lett., vol. 96, page 130502, 2006. (Cited on page 56.)

[Wakabayashi et al. 2015] Ryota Wakabayashi, Mikio Fujiwara, Ken-ichiro Yoshino, Yoshihiro Nambu, Masahide Sasaki and Takao Aoki. Time-bin entangled photon pair generation from Si micro-ring resonator. Optics Express, vol. 23, no. 2, page 1103, January 2015. (Cited on page 130.)

[Waks et al. 2002] Edo Waks, Assaf Zeevi and Yoshihisa Yamamoto. Security of quantum key distribution with entangled photons against individual attacks. Physical Review A, vol. 65, no. 5, page 052310, April 2002. (Cited on page 175.)

[Wang et al. 2011] H Wang, S Li, Z Xu, X Zhao, L Zhang, J Li, Y Wu, C Xie, K Peng and M Xiao. Quantum interference of stored dual-channel spin-wave excitations in a single tripod system. Phys. Rev. A, vol. 83, no. 4, page 43815, 2011. (Cited on page 104.) 
[Weier et al. 2011] Henning Weier, Harald Krauss, Markus Rau, Martin Fürst, Sebastian Nauerth and Harald Weinfurter. Quantum eavesdropping without interception: An attack exploiting the dead time of single-photon detectors. New Journal of Physics, vol. 13, 2011. (Cited on page 44.)

[Weihs et al. 1998] G. Weihs, T. Jennewein, C. Simon, H. Weinfurter and A. Zeilinger. Violation of Bell's Inequality under Strict Einstein Locality Conditions. Physical Review Letters, vol. 81, page 5039, 1998. (Cited on page 62.)

[Wiechers et al. 2011] C. Wiechers, L. Lydersen, C. Wittmann, D. Elser, J. Skaar, Ch Marquardt, V. Makarov and G. Leuchs. After-gate attack on a quantum cryptosystem. New Journal of Physics, vol. 13, 2011. (Cited on page 44.)

[Wilk et al. 2007] T. Wilk, S. C. Webster, H. P. Specht, G. Rempe and A. Kuhn. Polarization-controlled single photons. Physical Review Letters, vol. 98, no. 6, 2007. (Cited on page 127.)

[Williams et al. 2013] Robert J Williams, Ria G Krämer, Stefan Nolte and Michael J Withford. Femtosecond direct-writing of low-loss fiber Bragg gratings using a continuous core-scanning technique. Optics letters, vol. 38, no. 11, pages 1918-20, June 2013. (Cited on page 145.)

[Wootters \& Zurek 1982] W. K. Wootters and W. H. Zurek. A single quantum cannot be cloned, 1982. (Cited on page 25.)

[Xavier et al. 2009] G B Xavier, N Walenta, G de Faria, G P Temporão, N Gisin, H Zbinden and J P von der Weid. Experimental polarization encoded quantum key distribution over optical fibres with real-time continuous birefringence compensation. New J. Phys., vol. 11, no. 4, page 45015, 2009. (Cited on pages 36, 66, and 100.)

[Xiong et al. 2011] C Xiong, Christelle Monat, Alex S Clark, Christian Grillet, Graham D Marshall, M J Steel, Juntao Li, Liam O'Faolain, Thomas F Krauss, John G Rarity and Benjamin J Eggleton. Slow-light enhanced correlated photon pair generation in a silicon photonic crystal waveguide. Optics letters, vol. 36, no. 17, pages 3413-3415, 2011. (Cited on pages 65 and 130.) 
[Xiong et al. 2013] C Xiong, T D Vo, M J Collins, J Li, T F Krauss, M J Steel, A S Clark and B J Eggleton. Bidirectional multiplexing of heralded single photons from a silicon chip. Optics Letters, vol. 38, no. 23, pages 5176-9, 2013. (Cited on pages 130, 152, and 156.)

[Xiong et al. 2015a] C. Xiong, X. Zhang, Z. Liu, M. J. Collins, A. Mahendra, L. G. Helt, M. J. Steel, D. Y. Choi, C. J. Chae, P. H. W. Leong and B. J. Eggleton. Active Temporal Multiplexing of Indistinguishable Heralded Single Photons. page 13, August 2015. (Cited on page 137.)

[Xiong et al. 2015b] C. Xiong, X. Zhang, A. Mahendra, J. He, D.-Y. Choi, C. J. Chae, D. Marpaung, A. Leinse, R. G. Heideman, M. Hoekman, C. G. H. Roeloffzen, R. M. Oldenbeuving, P. W. L. van Dijk, C. Taddei, P. H. W. Leong and B. J. Eggleton. Compact and reconfigurable silicon nitride time-bin entanglement circuit. Optica, vol. 2, no. 8, page 724, August 2015. (Cited on page 130.)

[Xu et al. 2012] Feihu Xu, Bing Qi, Xiongfeng Ma, He Xu, Haoxuan Zheng and Hoi-Kwong Lo. Ultrafast quantum random number generation based on quantum phase fluctuations, 2012. (Cited on page 45.)

[Yamashita et al. 2013] Taro Yamashita, Shigehito Miki, Hirotaka Terai and Zhen Wang. Low-filling-factor superconducting single photon detector with high system detection efficiency. Optics express, vol. 21, no. 22, pages 27177-84, November 2013. (Cited on page 170.)

[Yan et al. 2011] Hui Yan, Shanchao Zhang, J. F. Chen, M. M T Loy, G. K L Wong and Shengwang Du. Generation of narrow-band hyperentangled nondegenerate paired photons. Physical Review Letters, vol. 106, no. 3, page 033601, 2011. (Cited on page 121.)

[Yang et al. 2006] Tao Yang, Qiang Zhang, Teng Yun Chen, Shan Lu, Juan Yin, Jian Wei Pan, Zhi Yi Wei, Jing Rong Tian and Jie Zhang. Experimental synchronization of independent entangled photon sources. Physical Review Letters, vol. 96, no. 11, 2006. (Cited on pages 57, 179, and 180.) 
[Yang et al. 2009] Jian Yang, Xiao Hui Bao, Han Zhang, Shuai Chen, Cheng Zhi Peng, Zeng Bing Chen and Jian Wei Pan. Experimental quantum teleportation and multiphoton entanglement via interfering narrowband photon sources. Physical Review A - Atomic, Molecular, and Optical Physics, vol. 80, no. 4, page 042321, October 2009. (Cited on page 179.)

[Yin et al. 2012] Juan Yin, Ji-Gang Ren, He Lu, Yuan Cao, Hai-Lin Yong, Yu-Ping Wu, Chang Liu, Sheng-Kai Liao, Fei Zhou, Yan Jiang, Xin-Dong Cai, Ping Xu, Ge-Sheng Pan, Jian-Jun Jia, Yong-Mei Huang, Hao Yin, Jian-Yu Wang, Yu-Ao Chen, ChengZhi Peng and Jian-Wei Pan. Quantum teleportation and entanglement distribution over 100-kilometre free-space channels. Nature, vol. 488, no. 7410, pages 185-188, 2012. (Cited on page 56.)

[Yoshino et al. 2012] K.-I. Yoshino, M Fujiwara, A Tanaka, S Takahashi, Y Nambu, A Tomita, S Miki, T Yamashita, Z Wang, M Sasaki and A Tajima. High-speed wavelength-division multiplexing quantum key distribution system. Opt. Lett., vol. 37, pages 223-225, 2012. (Cited on pages 71 and 102.)

[Yoshizawa et al. 2003] A. Yoshizawa, R. Kaji and H. Tsuchida. Generation of polarisationentangled photon pairs at $1550 \mathrm{~nm}$ using two PPLN waveguides. Electronics Letters, vol. 39, no. 7, page 621, 2003. (Cited on page 65.)

[Yuan et al. 2002] Zhiliang Yuan, Beata E Kardynal, R Mark Stevenson, Andrew J Shields, Charlene J Lobo, Ken Cooper, Neil S Beattie, David A Ritchie and Michael Pepper. Electrically driven single-photon source. Science (New York, N.Y.), vol. 295, no. 5552, pages 102-105, 2002. (Cited on page 127.)

[Zhang et al. 2007] Qiang Zhang, Xiuping Xie, Hiroki Takesue, Sae Woo Nam, Carsten Langrock, M M Fejer and Yoshihisa Yamamoto. Correlated photon-pair generation in reverse-proton-exchange PPLN waveguides with integrated mode demultiplexer at 10 GHz clock. Optics express, vol. 15, no. 16, pages 10288-10293, 2007. (Cited on page 157.)

[Zhang et al. 2015] X Zhang, I Jizan, J He, A S Clark, D-Y Choi, C J Chae, B J Eggleton and C Xiong. Enhancing the heralded single-photon rate from a silicon nanowire 
by time and wavelength division multiplexing pump pulses. Optics letters, vol. 40, no. 11, pages 2489-92, June 2015. (Cited on page 130.)

[Zhao et al. 2008] Yi Zhao, Chi Hang Fred Fung, Bing Qi, Christine Chen and Hoi Kwong Lo. Quantum hacking: Experimental demonstration of time-shift attack against practical quantum-key-distribution systems. Physical Review A - Atomic, Molecular, and Optical Physics, vol. 78, no. 4, 2008. (Cited on page 44.)

[Zhong et al. 2010] Tian Zhong, Franco N C Wong, Tony D Roberts and Philip Battle. High performance photon-pair source based on a fiber-coupled periodically poled KTiOPO(4) waveguide: erratum. Optics express, vol. 18, no. 14, page 20114, 2010. (Cited on page 64.)

[Zhou et al. 2013] Qiang Zhou, Wei Zhang, Tian-zhu Niu, Shuai Dong, Yi-dong Huang and Jiang-de Peng. A polarization maintaining scheme for $1.5 \mu \mathrm{m}$ polarization entangled photon pair generation in optical fibers. The European Physical Journal D, vol. 67, no. 9, page 202, sep 2013. (Cited on page 65.)

[Zukowski et al. 1993] M. Zukowski, A. Zeilinger, M. Horne and A. Ekert. "Event-readydetectors" Bell experiment via entanglement swapping, 1993. (Cited on pages 3 and 175.) 
